\title{
STRATEGIES FOR TARGETED AND IMAGE-GUIDED DRUG DELIVERY FOR SOLID TUMOR THERAPY
}

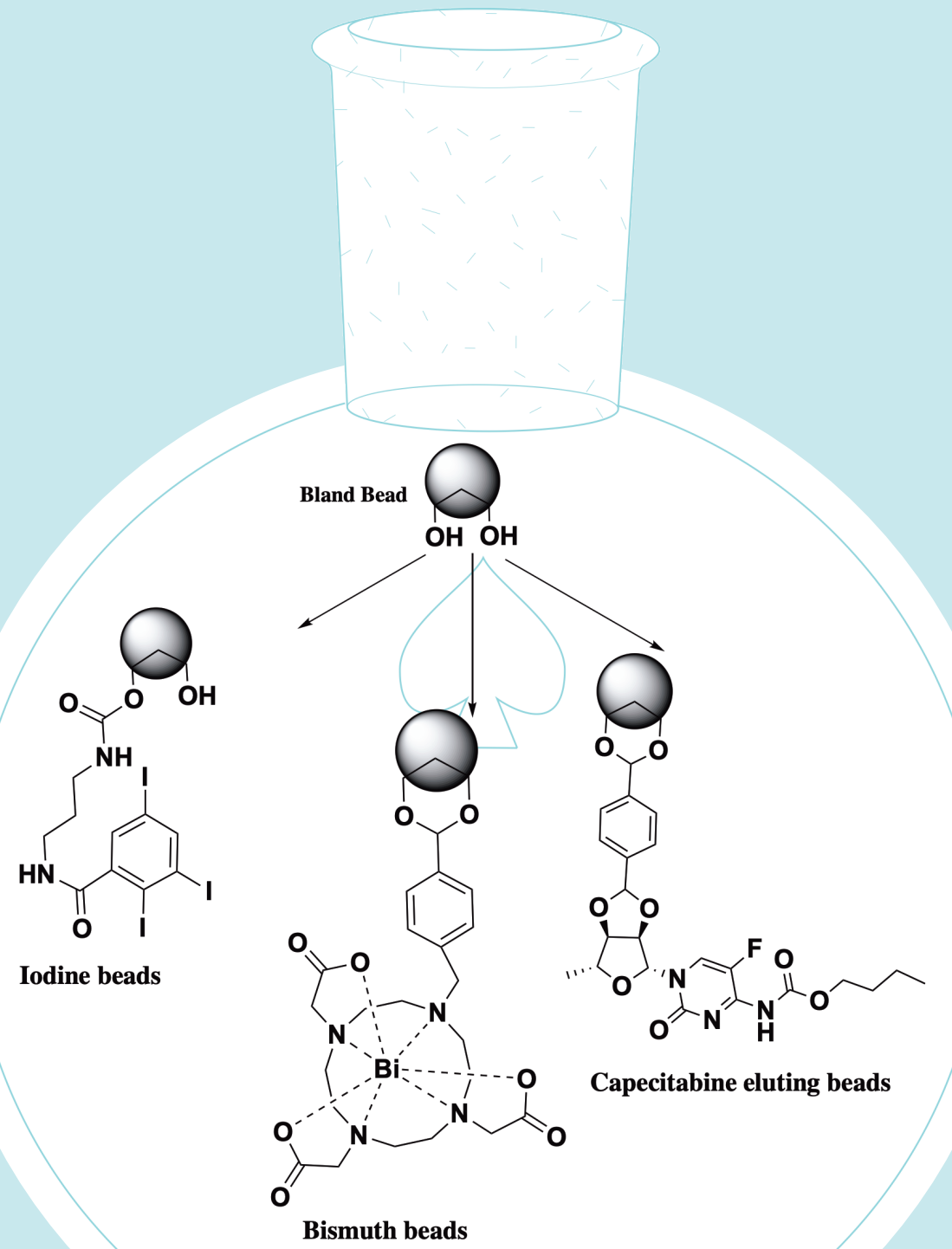




\section{STRATEGIES FOR TARGETED AND IMAGE GUIDED DRUG DELIVERY FOR SOLID TUMOR THERAPY}

Ayele Hailu Negussie 
The research described in this thesis was financially supported by the National Institutes of Health, Bethesda, MD, USA, and University of Twente Enschede, the Netherlands.

\section{Strategies for Targeted and Image-Guided Drug Delivery for Solid Tumor Therapy Ayele Hailu Negussie}

$\mathrm{PhD}$ thesis with references, with summaries in English and Dutch. University of Twente, Enschede, the Netherlands

Printed by: ProefschriftMaken || www.proefschriftmaken.nl

ISBN: 978-90-365-5183-0

DOI: $10.3990 / 1.9789036551830$

URL: https://doi.org/10.3990/1.9789036551830

C 2021 Ayele H Negussie, The Netherlands. All rights reserved. No parts of this thesis may be reproduced, stored in a retrieval system or transmitted in any form or by any means without permission of the author. Alle rechten voorbehouden. Niets uit deze uitgave mag worden vermenigvuldigd, in enige vorm of op enige wijze, zonder voorafgaande schriftelijke toestemming van de auteur.

Cover designed by Ayele H Negussie

Image on the cover page shows: images of imageable beads for image guided locoregional therapy and prediction of the use of boron-templated beads for TACE to deliver non-ionic drugs 


\title{
STRATEGIES FOR TARGETED AND IMAGE GUIDED DRUG DELIVERY FOR SOLID TUMOR THERAPY
}

\section{DISSERTATION}

\author{
to obtain \\ the degree of doctor at the Universiteit Twente, \\ on the authority of the rector magnificus, \\ Prof. dr. ir. A. Veldkamp, \\ on account of the decision of the Doctorate Board \\ to be publicly defended
}

on Wednesday 09 June 2021 at 14.45 hours

by

Ayele Hailu Negussie

born on the 28th January, 1966

in Addis Ababa, Ethiopia 
This dissertation has been approved by:

Supervisors:

Prof. dr. G. Storm University of Twente

Prof. dr. ir. C. Moonen University Medical Centre Utrecht

Referent:

Dr. B. J. Wood National Institutes of Health 


\section{Graduation Committee}

Chair / secretary: Prof. dr. J.L. Herek

Supervisors: $\quad$ Prof. dr. G. Storm

Prof. dr. ir. C. Moonen

Referent: Dr. B. J. Wood

Committee Members: Prof. dr. T.G.G.M.Lammers

Dr. S. Langereis

Prof. dr. D. W. Grijpma

Prof. dr. L. W. M. M. Terstappen 



\section{Table of Contents}

Chapter 1 General introduction: targeted and image-guided drug delivery for cancer therapy

Chapter 2 Formulation and characterization of magnetic resonance image-able thermally sensitive liposomes for use with magnetic resonance-guided high intensity focused ultrasound

Chapter 3 Synthesis and in vitro evaluation of novel cyclic NGR peptide targeted thermally sensitive liposome 75

Chapter 4 Synthesis and characterization of image-able polyvinyl alcohol microspheres for image-guided chemoembolization

Chapter 5 Synthesis, characterization, and imaging of radiopaque bismuth beads for image-guided transarterial embolization

Chapter 6 Summarizing discussion

Samenvatting

Abbreviations

List of publications

Acknowledgements

Curriculum Vitae 



\section{Chapter 1}

General introduction: targeted and image-guided drug delivery for cancer therapy 


\section{Cancer therapy}

Cancer is the second leading cause of death in the world with projected new cases and deaths of 21.6 million and 13.0 million, respectively, in the year $2030[1,2]$. The most effective treatment for primary cancer has been surgery alone or in combination with radiotherapy and/or conventional chemotherapy [3]. However, radiation and conventional chemotherapy expose patients to undesired side effects, and surgery is an invasive procedure, mostly indicated for the treatment of a localized tumor mass $[4,5]$. Typical characteristics of cancer cells, unlike normal cells, are dividing rapidly [6], infiltrating into normal tissues (invasion), penetrating blood/lymphatic vessels, then disseminating to distant sites (metastasize), as well as colonizing the new site and resisting apoptosis $[7,8]$. Progression of the disease is actively modulated by the tumor microenvironment (TME) [9-12], via a complex and variable array of molecular and sub-cellular processes, which play an important role in the immediate or eventual failure of many anticancer therapies [13-16]. Thus, the highly dynamic features of tumor cells and their microenvironment can prevent successful management of malignant disease and yet also present an opportunity for the development of new therapeutic strategies.

Locoregional cancer treatments augmented by image guided local drug delivery using either nanoscale Drug Delivery Systems (DDSs) like liposomes, or micrometer scale microbeads often referred to as Drug Delivery Devices (DDDs) [17-21] are emerging technologies for the treatment of various cancer. These treatments have demonstrated success in increasing intratumoral drug accumulation, reducing toxicity, as well as improving overall survival in preclinical models [17-20], clinical studies [17-20, 22-29], and in standard clinical practice $[20,28,30,31]$. The preferred locoregional cancer treatment strategies 
may include image-guided therapy and image-guided locoregional therapy in a growing number of therapeutic options [32]. They involve the use of medical imaging device/s to plan, implement, verify, monitor and evaluate therapeutic interventions and imageable DDSs and DDDs. One such approach involves systemic injection of drug delivery vehicles, e.g., thermosensitive liposomes (TSL), in combination with image guided thermal energy deploying techniques. These technologies include minimally invasive heat deploying devices such as radiofrequency $(\mathrm{RF})$, microwave (MW), or laser ablation procedures, as well as noninvasive high intensity focused ultrasound (HIFU). In one such paradigm, systemic injection of TSL (encapsulating the drug and/or contrast agent) leads initially to accumulation in the tumor by the so-called enhanced permeability and retention (EPR) (see Section 2.2) effect and/or receptor-mediated targeting. Then, deployment of thermal energy under computed tomography (CT), ultrasound, or magnetic resonance imaging (MRI) guidance can trigger TSL contents release and local delivery of drug and/or contrast agents in the tumor [22-24, 31, 33-39]. Conventional image-guided locoregional therapies include the use of minimally invasive ablative techniques and/or catheter-based transarterial delivery of, e.g., embolizing microparticles (with or without drug) under image guidance. These embolizing microparticles, suspended in clinical contrast medium, are injected using a microcatheter under fluoroscopic guidance to occlude tumor feeding vessels with the intent of tumor eradication for the treatment of, e.g., unresectable hepatocellular carcinoma (HCC) [40-42]. The therapeutic effect can be further increased by using embolizing microparticles which are tailored to include chemoor radiotherapeutic agents [28, 29]. 


\section{Drug-delivery systems and drug-delivery devices}

Chemotherapy is an option for localized as well as metastasized tumors [3], involving oral or systemic administration (via intravenous injection) of a therapeutic agent or combination of agents. Such chemotherapy protocols have demonstrated the ability to improve progression and disease-free survival in some patients but often not without serious toxicity to healthy organs and tissues [43, 44]. To improve on the clinical situation, systemic injection of drug delivery systems (DDS) combined with thermal energy application at the diseased site, as well as drug-delivery devices for locoregional treatments have been shown to improve pharmacokinetics and biodistribution of therapeutic agents, thereby maximizing treatment efficacy and reducing toxicity [45-47].

DDSs contain drugs and/or imaging agents by encapsulation, micellization or covalent conjugation. They can be tailored to deliver drug preferentially to the diseased site by designing them with the help of nanotechnology in combination with minimally or noninvasive hyperthermia applicators, such as RFA needles and HIFU. Such nanosized DDS are often administered intravenously and can be tailored to optimally (1) target tumor cells, tumor vasculature or the TME, (2) circulate in the bloodstream for extended periods of time, (3) release contents when triggered by stimuli and/or upon endo/pinocytosis by target cells, and 4) report on accumulation of DDS at the target $[45,48-51]$. In this thesis research, the focus is particularly on the development of the so-called thermosensitive nanoparticles, which can be triggered to release their drug content by exposure to increased temperatures, created by e.g., RFA needles or HIFU; and imageable drug delivery devices for image-guided locoregional therapy. 
DDS can be prepared from natural or synthetic lipids and polymers (biocompatible) to produce vesicular and/or spherical structures such as liposomes, micelles, and polymersomes at nanometer scale [52-55]. DDS can be used to i) load drug/ contrast agent into them, ii) have targeting agents on their surface, and/or iii) covalently attach drug/contrast agent to facilitate tumor-specific targeting [56-58].

Drug-delivery devices (DDDs) are commonly prepared from biocompatible and/ or biodegradable polymeric materials in the form of calibrated microparticles (beads with defined size ranges) in micrometer scale and used to occlude blood vessels feeding tumors with the intent of tumor eradication. To augment their antitumor effects, they can also be loaded with chemotherapeutics, for example by utilizing ion exchange mechanisms $[20,21,59,60]$. In addition, beads can be tailored to have specific micron sizes in order to optimally occlude blood vessels with differently sized diameters (proximal or distal) feeding the tumor, and further functionalized for loading therapeutic agents, covalently binding of imaging agents and/or physically hold contrast agents in their pores [61, 62]. The mode of delivery of DDDs is via a locally applied catheter.

Liposomes form vesicles spontaneously when phospholipids and other constituent components are hydrated with aqueous medium. A variety of liposomes loaded with therapeutic and/or imaging agents have been reported in the literature for drug delivery and imaging applications $[63,64]$. In general, the hydrated core (aqueous lumen) is utilized as a booth for hydrophilic drugs while the phospholipid bilayers serve as compartment for the association of amphiphilic and hydrophobic drugs. Incorporation of phospholipids modified with polyethylene glycol (PEG) and phospholipid-PEG-ligands into liposomal bilayer membranes has produced a variety of long-circulating liposomal (stealth) formulations with tumor and TME -targeting ability, with prolonged circulation time characteristics by delayed 
uptake by the reticuloendothelial system in particular the liver (Kupffer cells) and the spleen (splenic macrophages). [47].

In this introductory chapter, the rationale and foundational background for the use of nanoparticles for targeted drug delivery, as well as micron-scale drugdelivery devices for locoregional delivery will be presented with emphasis on 1) thermosensitive, intravenously administered liposomes for tumor-selective delivery, and 2) drug-delivery devices in the form of image-able microparticles (beads) for blocking tumor-feeding vessels and for loco-regional treatment. The unifying theme is nano- and micro-scale drug delivery devices (DDD) and drug delivery systems (DDS) with image-ability allowing image guided drug delivery. Such systems have broad potential for the optimization of image-guided minimally invasive therapies, which have yet to fully take advantage of rational and closedloop image-guided drug delivery.

\section{Nanoparticles for tumor-targeted drug delivery}

Targeted drug delivery was conceptualized after Paul Ehrlich's discovery of selective staining of gram-positive bacteria with a dye - a targeting concept resulting from the correlation of a molecule's chemical structure with its selective action on different cellular substrate [65]. Recently, substantial progress has made in material engineering that enable targeted delivery of drugs by exploiting the unique pathobiological features of the tumor or TME [66-68]. These pathobiological features relate to cancer metabolism [69], infiltrating immune system [70], molecular markers that are overexpressed in or unique to the tumor and tumor microenvironment [71], and permeability of the intratumoral blood vessels (enabling the EPR effect). [72, 73]. Indeed, the multi-scale junction and interface 
between chemistry and biology is a fertile territory for novel multi-modal drug delivery approaches.

\section{The EPR effect: Passive targeting}

Tumor tissue is often characterized by a poorly developed lymphatic drainage system with vasculature composed of vessels which show increased permeability, as compared to blood vessels with continuous endothelium walls present in most healthy tissues [9]. This increased permeability allows the selective passage of certain sized nanoparticles into the tumor interstitial tissue [46]. Ideally, the long circulation time fosters extravasation through the hyperpermeable tumor vasculature [74-76] due to the presence of gaps (400-1000 nm) between the endothelial cells lining the tumor vessels [77]. Tailored surface modification and/ or suitable composition (e.g. polyethylene glycol (PEG) coating) can endow them with 'stealthiness' to the immune system allowing them to circulate long enough to extravasate through the gaps in the tumor endothelial linings - commonly referred to as passive targeting [78-80]. The Food and Drug Administration (FDA) and European Medicines Agency (EMA) have approved a number of passively targeted liposomal drug formulations for the treatment of different cancers (Table 1) [81-84], and some more advanced DDS are in various stages of development and in clinical trials [85-87]. The goal is often reduction in systemic (potentially toxic) doses, while delivering doses locally or regionally effectively, resulting in "broadening" the therapeutic window. Passive accumulation of nanoparticles, although valuable for widening the therapeutic window of the encapsulated drugs, may not provide satisfying antitumor responses, as drug release from such nanoparticles can be slow and incomplete $[72,80]$. In fact, this concern has motivated us for carrying out the research presented in this thesis. More sophisticated and engineered targeted drug delivery technologies that result in 
Table 1: Examples of FDA and EMA -approved nanoparticle-based DDS for intravenous cancer therapy [81-84]

\begin{tabular}{|c|c|c|c|}
\hline Trade Name & Generic Name & Indications & Benefit \\
\hline Doxil $^{\oplus}$ & $\begin{array}{l}\text { Liposomal } \\
\text { Doxorubicin }\end{array}$ & $\begin{array}{l}\text { Kaposi's sarcoma, } \\
\text { Ovarian cancer, } \\
\text { multiple myeloma }\end{array}$ & $\begin{array}{l}\text { Increased tumor delivery } \\
\text { decreased } \\
\text { toxicity compared to free drug }\end{array}$ \\
\hline Onivyde ${ }^{\circledast}$ & $\begin{array}{l}\text { Liposomal } \\
\text { Irinotecan }\end{array}$ & Pancreatic cancer & $\begin{array}{l}\text { Increased tumor delivery to } \\
\text { diseased site, decreased toxicity } \\
\text { compared to free drug }\end{array}$ \\
\hline Abraxane $e^{\circledast}$ & $\begin{array}{l}\text { Albumin-bound } \\
\text { paclitaxel }\end{array}$ & $\begin{array}{l}\text { Breast cancer, Non } \\
\text { small-cell lung } \\
\text { cancer, Pancreatic } \\
\text { cancer }\end{array}$ & $\begin{array}{l}\text { Solubilization, increased tumor } \\
\text { delivery, decreased toxicity }\end{array}$ \\
\hline DaunoXome ${ }^{\oplus}$ & $\begin{array}{l}\text { Liposomal } \\
\text { Daunorubicin }\end{array}$ & Kaposi's sarcoma & $\begin{array}{l}\text { Increased tumor delivery, } \\
\text { decreased } \\
\text { toxicity }\end{array}$ \\
\hline Genexol-PM $^{\circledast}$ & $\begin{array}{l}\text { Polymeric } \\
\text { micelles } \\
\text { containing } \\
\text { Paclitaxel }\end{array}$ & $\begin{array}{l}\text { Breast cancer, Non } \\
\text { small-cell lung } \\
\text { cancer }\end{array}$ & Solubilization, decreased toxicity \\
\hline Marquibo $^{\circledast}$ & $\begin{array}{l}\text { Liposomal } \\
\text { Vincristine }\end{array}$ & $\begin{array}{l}\text { Acute } \\
\text { lymphoblastic } \\
\text { leukemia }\end{array}$ & $\begin{array}{l}\text { Enhanced tumor delivery, } \\
\text { decreased } \\
\text { toxicity }\end{array}$ \\
\hline Myocet $^{\oplus}$ & $\begin{array}{l}\text { Liposomal } \\
\text { Doxorubicin }\end{array}$ & $\begin{array}{l}\text { Metastatic breast } \\
\text { cancer }\end{array}$ & $\begin{array}{l}\text { Enhanced tumor delivery, } \\
\text { decreased toxicity }\end{array}$ \\
\hline Mepact $^{\circledast}$ & $\begin{array}{l}\text { Liposomal } \\
\text { Mifamurtide }\end{array}$ & Osteosarcoma & Improved survival \\
\hline
\end{tabular}

higher drug concentrations at the diseased site are discussed in the following sections $[72,80]$.

\section{Stimuli-responsive or ligand targeting: active targeting}

Similar to passively targeted nanoparticles, actively targeted nanoparticles also aim to enhance the drug amount in the tumor. However, the latter are designed to release their contents either by receptor-mediated endo/pinocytosis (via binding to overexpressed receptors) or by altering their chemical and/or physical properties 
upon stimulant exposure e.g., light, $\mathrm{pH}$, ultrasound, enzymes, or temperature [88, 89] to overcome limitations of passively targeting drug delivery. Nanoparticles modified on their surface with targeting ligands (e.g., antibodies, peptides, aptamers) are called ligand-targeted nanoparticles. These modifications are designed to promote the nanoparticle binding to receptors (over)expressed by or unique to cells in the tumor or TME (e.g., tumor cells, endothelial cells, immune cells) $[67,90-92]$. In comparison to ligand-mediated targeting, stimuli-responsive nanoparticles provide instantly bioavailable drug upon nanoparticle exposure to stimuli. Such actively targeted nanoparticles in combination with energy deploying therapy devices serve to overcome the limitation of low intratumoral drug release often associated with nanoparticles that only rely on passive tumor targeting via the EPR effect.

\section{Stimuli-responsive targeting nanoparticles}

Advances in material chemistry, device engineering, as well as breakthroughs in the molecular understanding of cancer have led to the development of stimuliresponsive drug delivery nanoparticles. Stimuli-responsive liposomes, micelles, and polymersomes exhibit a sharp change in their structural integrity upon a modest threshold change in the tumor environment $[93,94]$. Consequently, their drug payload can be rapidly released, and the dose delivered in a spatiotemporally controlled fashion [95-97]. For example, thermosensitive liposomes (Figure 1.1) are stable at body temperature $\left(37^{\circ} \mathrm{C}\right)$ but respond to temperatures $>40{ }^{\circ} \mathrm{C}$ by rapidly releasing their payload to the heated tumor [98-101]. A preclinical study conducted with doxorubicin-containing non-thermosensitive liposomes (Dox-NTL) and thermosensitive liposomes (Dox-TSL) in combination with local heating demonstrated a 5.6-fold enhancement in intratumoral drug accumulation as well as longer tumor growth delay in mice treated with Dox-TSL [102]. 

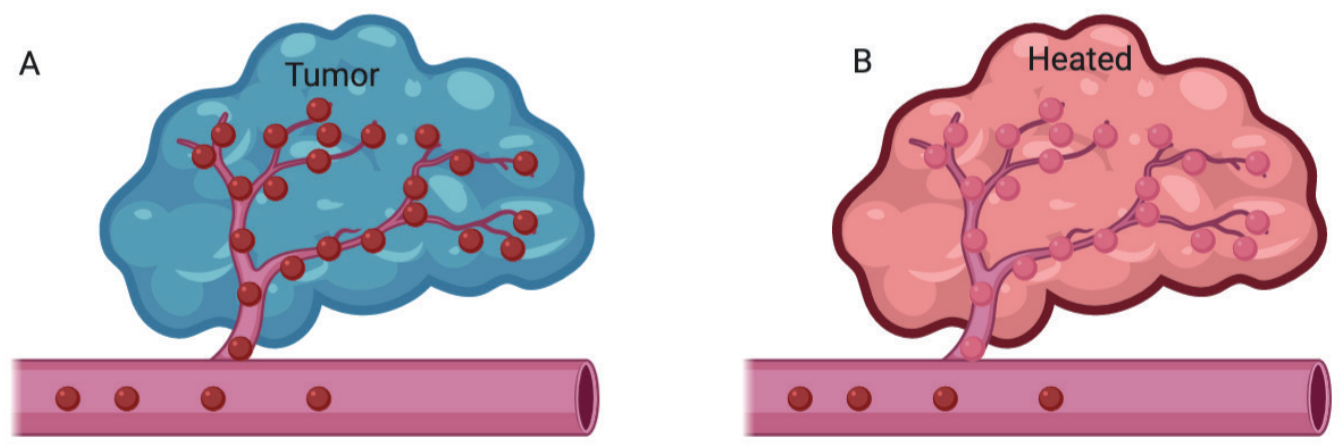

Figure 1.1 Temperature sensitive liposomes extravasate in the tumor: A) at physiological temperature, $37^{\circ} \mathrm{C}$ and $\mathrm{B}$ ) releasing their content up on mild hyperthermia (heating).

A separate preclinical study conducted by Needham et al. utilized a human tumor xenograft model and doxorubicin-containing thermosensitive liposomes in combination with local tumor heating. The results of this study show that the achieved high intratumoral drug concentration correlates with biological outcomes of complete tumor regression and enhanced disease free survival [103], demonstrating that nanoparticles with targeting via local deployment are efficacious in vivo and may reduce toxicity to healthy tissues in preclinical model. More recent studies have shown that doxorubicin-containing thermosensitive liposomes in conjunction with thermal HIFU resulted in enhanced drug delivery to the heated tumor compared to unheated organs [104-110], and a markedly enhanced delivery when heat plus carrier in tumor is compared to free drug and no heat in normal tissue.

HIFU is a minimally or non-invasive technique used for pain control and tumor tissue destruction (heat-induced ablation), and has been in clinical use since the late 1940s [111]. Since then, various HIFU devices have been FDA-approved for, e.g., ablation of prostatic tissue [112], treatment of uterine fibroids [113], pain palliation of bone metastasis -related pain [114], and for essential tremor [115]. 
The use of HIFU, particularly when coupled with MRI (MR-HIFU), has shown wide clinical applications [116]. MR-HIFU provides the ability to select target areas, monitor drug delivery and temperature changes [116-118], evaluate therapy outcome [119], as well as assure treatment safety via real-time imaging guidance [120]. HIFU can also be used to selectively and noninvasively heat target tissue to $40-45^{\circ} \mathrm{C}$ (mild hyperthermia) for a prolonged period of time ( $>10 \mathrm{~min}$ ), as a "non-invasive interstitial hyperthermia" [107]. When HIFU-mediated mild hyperthermia is combined with therapeutic agents, they act in synergy for tumor cell death [24]. In addition, HIFU can trigger drug release from thermosensitive liposomes, and in conjunction with MRI-based temperature monitoring provides the combined benefits of thermotherapy and chemotherapy [24, 118].

Clinical trials utilizing TSLs in cancer therapy have documented encouraging results [22, 121-125]. Despite the usefulness of stimuli-responsive targeting nanoparticles, however, no regulatory-approved stimuli-responsive DDS are currently available for clinical use. In this thesis, the development of novel, imageable liposomes for MR-HIFU-mediated drug delivery and optimization thereof are presented in Chapter 2.

\section{Ligand-targeted nanoparticles}

Ligand-targeted nanoparticles involve the use of targeting moieties conjugated onto the surface of the nanoparticles, which bind to receptors overexpressed on or specific to target cells (Figure1.2) in the tumor or tumor vascular endothelium, followed by cellular internalization due to receptor-mediated endocytosis [126].

The most common types of this active targeting strategy are: 1) targeting the tumor cells for cellular internalization of drug-containing nanoparticles, and 2) 


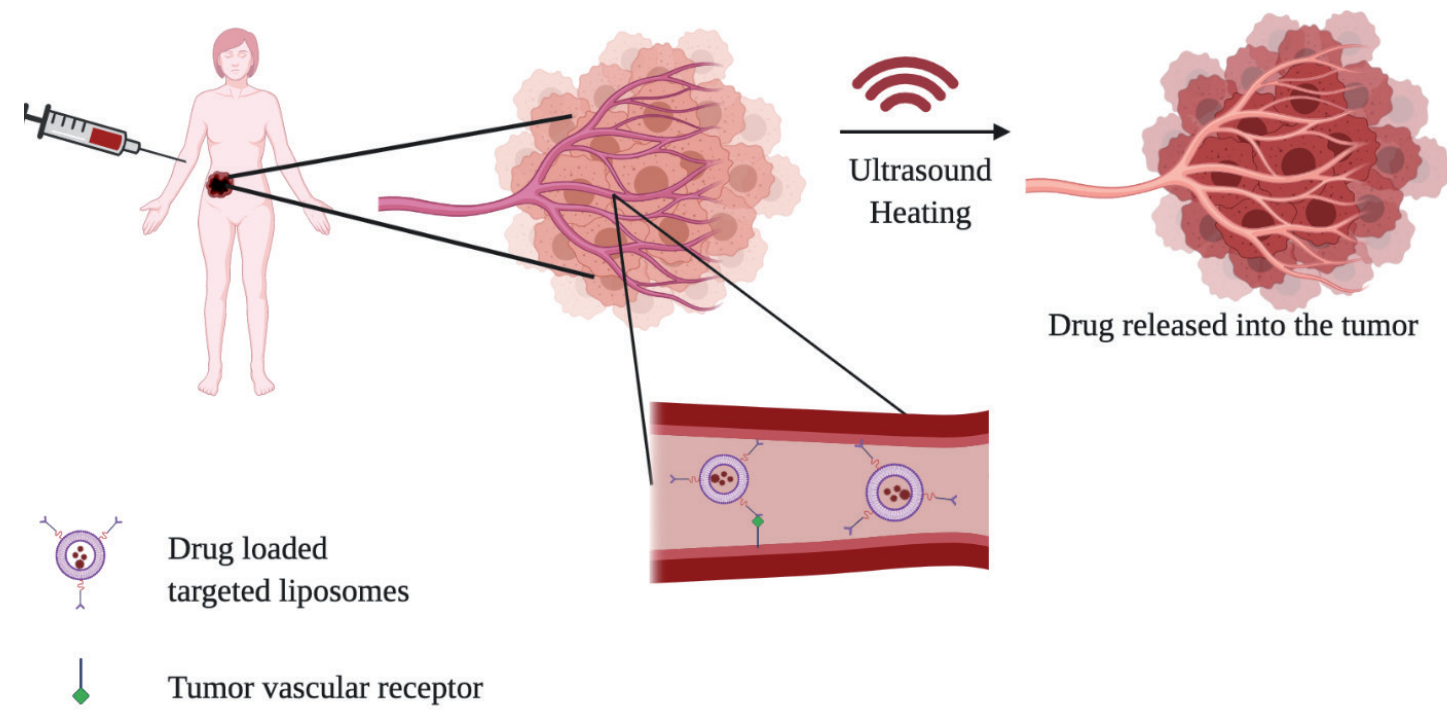

(8) Receptor-liposome binding

Figure 1.2 Intravenously injected targeted thermosensitive liposomes reach the tumor vasculature and bind to tumor vascular receptors. As a result, the liposomes in the tumor vasculature present for a longer period of time allowing for activation by an external hyperthermia applicator, e.g., ultrasound heating, and release their content within the tumor.

those targeting tumoral endothelium to destruct tumoral vascular network so that the tumors die off by lack of nutrients and oxygen [127, 128]. Commonly utilized receptors on tumor cells include folate, glycoproteins, epidermal growth factor, and transferrin [129-140]. Similarly, receptors on tumor endothelial cells include vascular endothelial growth factor receptors 1 and 2 [129, 141, 142], the endothelial cell receptor $\alpha_{V} \beta_{3}$ [143], vascular cell adhesion molecule-1 [144], and the matrix metalloproteinases [145-148].

Even though receptor-mediated DDS are not standard in clinical use yet, promising preclinical and early clinical results have been reported [149]. For example, doxorubicin-containing nano systems, such as dual-targeted (AS1411 aptamer and folic acid), $\mathrm{pH}$-sensitive biocompatible polymeric nanoparticles 
[150] and tumor-specific monoclonal antibody-targeted liposomes [151, 152], provided enhanced cellular uptake and greater cancer cell killing while sparing noncancerous cells. In this thesis, the development of tumor endothelium-targeted liposomes with enhanced affinity is presented in Chapter 3.

\section{Drug-delivery devices}

Drug-delivery devices are tools used to deliver drugs locally in a controlled fashion. These devices include medical implants, drug-eluting stents, and drug-eluting beads $[20,153]$. Drug eluting beads are spherical hydrogels made from various polymeric materials and used, e.g., as an embolic agent to treat cancer [154]. The beads can be tailored to a range of specific sizes $(70-700 \mathrm{~mm})$, made to load drug using electrostatic interaction between the negative charge in the beads and the positive charge on the drug (Figure 1.3) and applied intra-arterially to block vessels (transarterial embolization-TAE), and/or deliver drugs (transarterial chemoembolization-TACE) to selected tumor-feeding vessels [20, 155-159]. These procedures require the use of microcatheters to deliver beads under fluoroscopy guidance - as a form of loco-regional image-guided drug delivery (Figure 1.4).

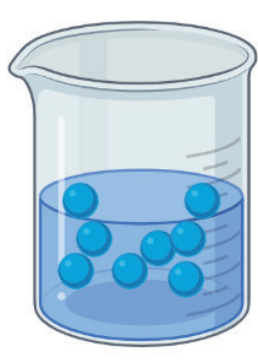

Beads

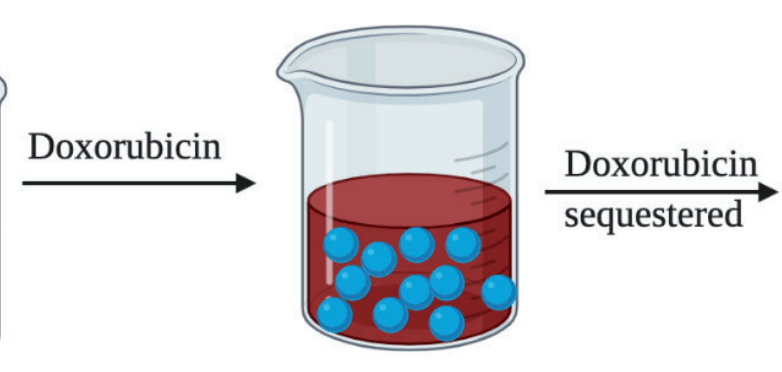

Beads + Drug solution

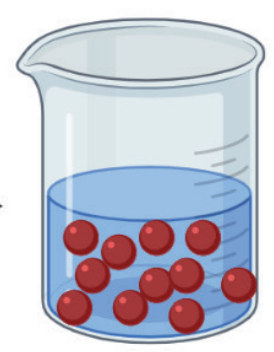

Drug loaded Beads

Figure 1.3 The process of drug loading (a process called cationic-anionic interaction) into drug eluting beads-DDDs. 


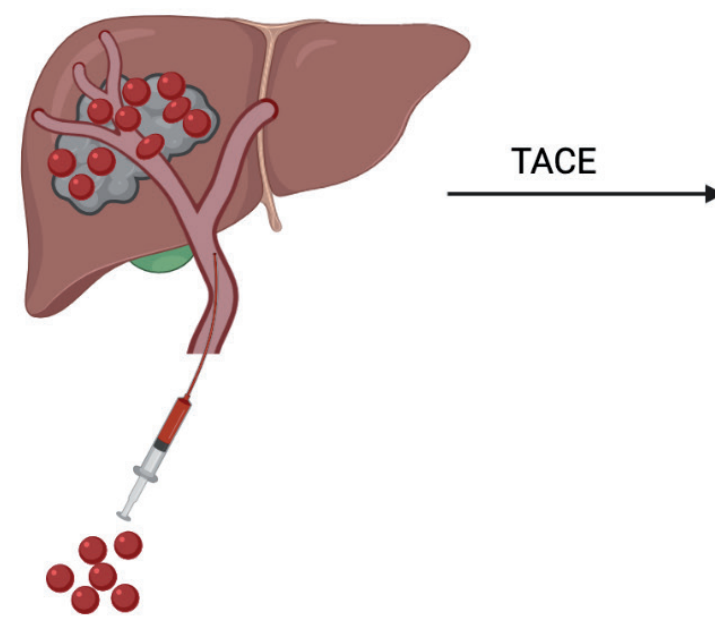

Drug eluting bead infusion (TACE)

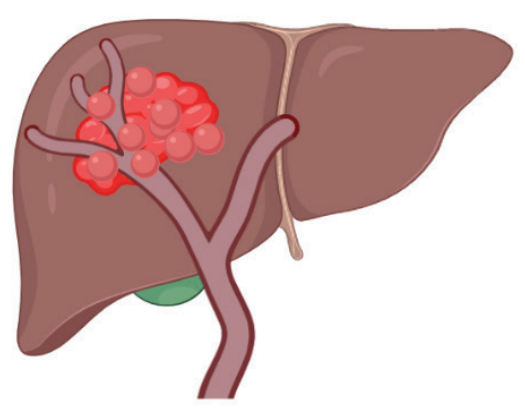

Drug eluted from the beads into tumor

Figure 1.4 4 Locoregional treatment of liver cancer using drug loaded beads-DDDs. A procedure known as transarterial chemoembolization (TACE).

Loco-regional drug delivery represents an approach to minimize systemic toxicity of chemotherapeutics by administering drugs loco-regionally, i.e. directly in or around tumors, where it is needed most [160].

The concept of loco-regional drug delivery was pioneered and advanced by Klopp et al. [161, 162] and others [160] for the management of various neoplasms using arterial administration of nitrogen mustard. Widely accepted routes of administration for loco-regional drug delivery are intra-arterial (IA), intraperitoneal (IP), intra-tumoral, and intra-thecal (IT) [160]. For example, intraarterial infusion of therapeutic agents is now routinely used for the treatment of various tumors [160] and can provide up to 50 times higher intratumoral drug levels compared to systemic therapies [163]. In settings where systemic treatment of micro metastases is desired, then local or regional DDS may not be ideal.

Recent advances in microcatheter technology, navigation tools and technology for selective delivery, and the application of drug-eluting beads (DEBs) combined 
with imaging techniques might improve anticancer efficacy and safety profile of loco-regional drug delivery platforms, although there is no consensus reached and no standardization yet achieved for its technical use (physician dependent). Micro-sized DDDs containing doxorubicin can be used to treat locoregionally confined primary liver cancer to improve patient survival. There is data that such an aggressive local-regional approach adds value $[62,164]$ in mapping drug distribution across treated zone. In this approach, insertion of a microcatheter within a tumor feeding artery, and delivery of tailored, drug-containing beads suspended in contrast media is performed under fluoroscopy and Cone Beam Computed Tomography (CBCT) guidance. Intra-arterial infusion of beads as an embolic agent, or for chemotherapy or radiotherapy purposes (chemo- or radioembolization), is shown to be useful in clinical practice particularly for primary hepatic tumor lesions after failure of surgery (first line) or systemic chemotherapy (second line) $[165,166]$. Beads occlude the tumor-feeding artery partially or completely, leading to the tumor starving to oxygen and nutrients. Since the beads can hold a considerable amount of drug (e.g., $36 \mathrm{mg}$ of doxorubicin per $1 \mathrm{~mL}$ of wet beads), they can deliver a high dose to the nearby lesions in a sustained fashion. One clear disadvantage of this technology is the difficulty of evaluating bead deposition post intra-arterial infusion, as the beads cannot be imaged. To address this drawback, image-able beads were developed using an FDA-approved embolic material with radiopacifiers (Iodine or Bismuth) for CT-guided TAE or TACE. This development is described in Chapters $\mathbf{4}$ and 5 of this thesis. The iodinated version has been FDA cleared for clinical use as an embolic but remains off-label and is only available for investigational use. 


\section{Loco-regional drug delivery using image-able drug-eluting beads}

Non-invasive imaging is used to report the location of the drug-loaded imageable beads in and around the target lesion and estimate the amount of delivered drug $[164,167]$. Besides, imaging helps the physician to decide when to end treatment, to evaluate under-treated tumor areas, and to assess outcome. Thus, local therapy can be optimized, off-target exposure and related toxicity minimized, and untreated tumor identified for subsequent treatment [164, 167]. In Chapter 4, the development of iodine-based beads for CT-guided TACE is described. Such beads are useful in assessing the quality of embolization and aid in predicting delivered drug by using imaging post TACE. However, the contrast media contains the same radiopacifier as the bead suspension, and therefore the beads cannot readily be differentiated from the suspending contrast agent using CBCT (post TACE). This limitation indicates the need for image-able beads that are distinguishable from the suspending liquid, and thus can provide useful information such as effectiveness of the treatment during and post TACE. This can be realized by utilizing dual energy CT (DECT) together with the new imageable beads. DECT is an emerging imaging modality currently in clinical use. It is a computed tomography technique, that uses, in principle, two different energy sources, for a high-energy spectrum around $140 \mathrm{kV}$ and a lower-energy spectrum at 80 or $100 \mathrm{kV}$ and two detectors that are potentially able to distinguish different materials based on their k-edge X-ray absorption characteristics. k-edge X-ray absorption is a characteristic energy manifested when X-rays energy absorbed just above the binding energy leading to the ejection of the innermost electron of a given atom with a characteristic wavelength [168]. DECT utilizes material k-edge characteristics to capture images in two energy bins $(140 \mathrm{kV}$ and 80/100 $\mathrm{kV}$ ) to distinguish different material components using the two different detectors. 
Therefore, by developing a new radiopacifier-containing bead, the unmet needs of TACE - which is the inability of follow up post TACE - will be met. This technology further offers the possibility of delivering dual drugs which have a synergistic effect combined in one 'paired' system with the ability of quantifying each drug separately from the other from the imaging data using DECT. The development of Bismuth-based microparticles to be used with DECT for imageguided TACE is depicted in Chapter $\mathbf{5}$.

Standard clinical practice does not take full advantage of the possibilities for image guidance, nor of the opportunity to perform drug dosimetry and/or quantification and localization of drug molecules delivered via a paired imageable delivery system. The main reason is that the current imageable beads and the clinical contrast agent are comprised of the same radiopacifier which impedes the differentiation of the bead from the contrast agent. The challenge addressed in this thesis if therefore to develop microbeads with a different radiopacifier. Such new radiopacifier microbeads with drug loading capability can be used to monitor the localization of the dual beads and to quantify the delivered drugs to adjust drug dosimetry.

Taken together, clearly the nano DDS and micro scaled DDD may be applied to many of the problems inherent to image guided cancer therapy.

\section{Aim and outline of the thesis}

The general aims of this thesis are: 1) to develop and characterize thermosensitive liposomes and evaluate their utilization in combination with heating devices for targeted and image-guided drug delivery and 2) to synthesize and characterize imageable microparticles as a drug-delivery device for image-guided loco-regional 
drug delivery. Both these technologies, thermosensitive drug delivery systems and drug-delivery devices, are designed to: i) improve the opportunities to localize drug delivery and assess performance while enabling to inform rational drug selection based on different formulations and deliver and monitor the drugs within the tumor microenvironment. Even though not part of this thesis, the in vivo and clinical validation of these tools have been described [169-171], and ii) locally release their contents and deliver high doses of drug in a minimally invasive manner. For example, to achieve tumor-targeted drug delivery, intravenously administered liposomes can be designed to target overexpressed and tumor-distinct receptors and/or to exploit leaky tumor vasculature which is often known as the enhanced permeability and retention (EPR) effect $[73,172,173]$. In addition, microparticles (beads) can be used for blocking blood and nutrient supply to the tumor to enable loco-regional delivery of the therapeutic agent to achieve tumor eradication (drug delivery device).

This thesis is organized into six chapters. Chapter $\mathbf{1}$ serves as an introduction to the thesis contents. The second and third chapters deal with nanoparticles for stimuli-targeted and vascular-targeted delivery of therapeutics to a tumor. Chapter 2 specifically focuses on the development and characterization of imageguided thermosensitive liposomes for focused ultrasound-mediated drug delivery. The hypothesis is that imageable thermosensitive liposomes enable real time monitoring and on-demand content release of the liposomes within tumor tissue using magnetic resonance high intensity focused ultrasound (MR-HIFU). The work focused on the preparation and characterization of a novel MR-imageable thermosensitive liposome formulation containing both the antitumor drug doxorubicin and the MR contrast agent Gd-HP-DO ${ }_{3} \mathrm{~A}$. Real time monitoring of liposomal content release and contrast agent spatial distribution were studied using MR-HIFU in tissue-mimicking phantoms as well as in vivo. Chapter 3 
focuses on the synthesis of a cyclic NGR peptide and design of thermosensitive liposomes exposing this peptide on their surface for tumor vasculature-targeted drug delivery. The NGR peptide consists of asparagine $(N)$, glycine $(G)$, and arginine (R) motifs, is conjugated to DSPE-PEG-amine (one of the constituents of the liposomes) either as linear or cyclic peptide and incorporated into liposomes to obtain linear of cyclic NGR-decorated thermosensitive liposomes. These liposomes are intended to target CD13/ aminopeptidase N (APN) receptors often overexpressed on tumor endothelial cells. The hypothesis here is that, unlike the thermosensitive liposomes without targeting ligand, the NGR-decorated thermosensitive liposomes are able to bind to endothelial cells in the blood vessels in the tumor expressing CD13/APN receptors, with the potential of delivering high local drug doses instantly upon heating. Chapters $\mathbf{4}$ and $\mathbf{5}$ focus on the use of clinically approved microparticles for the development of a novel image-able microparticle-based drug-device combination for transarterial chemoembolization of liver tumors. The microparticles currently used in the clinic for this purpose are non-imageable drug eluting beads for transarterial chemoembolization (DEBTACE). These microparticles contain reactive hydroxyl groups which in this thesis are exploited for the covalent attachment of imaging agents. In Chapter 4, an iodine containing moiety was attached to these microparticles through acetal linkages, and these microparticles were subsequently evaluated for use as image-able DEB-TACE. The hypothesis is that the imageable beads report on drug delivered to the targeted branches of arteries of the diseased liver as well as inform on the extent of embolization post TACE. In Chapter 5, a bismuthchelated macrocycle was covalently bound to the microparticles and used as radiopacifier. Similar to iodinated beads, the bismuth beads enable monitoring of the location and amount of delivered drug, as well as the extent of embolization post TACE. However, as opposed to iodinated beads, treatment based on bismuth beads provides sufficient differentiation from liquid contrast post-TACE using 
imaging data obtained from DECT. Chapter $\mathbf{6}$ summarizes the results of the thesis and provides perspectives with special emphasis on new applications of microparticles for loco-regional drug delivery. Special attention is given to the preparation of novel engineered microparticles containing drugs to be applied for TACE of hepatocellular carcinoma (HCC) to achieve superior antitumor effects as compared to those obtained with the current drug-eluting beads. 


\section{References}

1. Torre, L.A., et al., Global cancer statistics, 2012. CA Cancer J. Clin. 2015, 65(2): p. 87-108.

2. American Cancer Society. Cancer Facts \& Figures 2017; Available from: https://www.cancer. org/content/dam/cancer-org/research/cancer-facts-and-statistics/annual-cancer-facts-andfigures/2017/cancer-facts-and-figures-2017.pdf.

3. Arruebo, M., et al., Assessment of the evolution of cancer treatment therapies. Cancers, 2011. 3: p. 3279-3330.

4. Urruticoechea, A., et al., Recent advances in cancer therapy: an overview. Curr. Pharm. Des., 2010. 16(1): p. 3-10.

5. Baskar, R., et al., Cancer and radiation therapy: current advances and future directions. Int. J. Medical Sci., 2012. 9(3): p. 193-199.

6. Joyce, J.A., Therapeutic targeting of the tumor microenvironment. Cancer Cell, 2005. 7(6): p. 513-20.

7. Al-Hajj, M. and M.F. Clarke, Self-renewal and solid tumor stem cells. Oncogene, 2004. 23(43): p. 7274-7282.

8. Hanahan, D. and R.A. Weinberg, Hallmarks of cancer: the next generation. Cell, 2011. 144(5): p. 646-674.

9. Hanahan, D. and R.A. Weinberg, The hallmarks of cancer. Cell, 2000. 100(1): p. 57-70.

10. Mbeunkui, F. and D.J. Johann, Jr., Cancer and the tumor microenvironment: a review of an essential relationship. Cancer Chemother. Pharmacol., 2009. 63(4): p. 571-82.

11. Tsai, M.-J., et al., Tumor microenvironment: a new treatment target for cancer. ISRN Biochem., 2014: p. 351959/1-351959/8.

12. Al-Akra, L., et al., The biochemical and molecular mechanisms involved in the role of tumor micro-environment stress in development of drug resistance. Biochim. Biophys. Acta, Gen. Subj., 2019. 1863(9): p. 1390-1397.

13. Munson, J.M. and A.C. Shieh, Interstitial fluid flow in cancer: implications for disease progression and treatment. Cancer Manag. Res. 2014. 6: p. 317-328.

14. Navalitloha, Y., et al., Therapeutic implications of tumor interstitial fluid pressure in subcutaneous $R G$-2 tumors. Neuro-Oncol., 2006. 8(3): p. 227-233.

15. Son, B., et al., The role of tumor microenvironment in therapeutic resistance. Oncotarget, 2017. 8(3): p. 3933-3945.

16. Yuan, Y., et al., Role of the tumor microenvironment in tumor progression and the clinical applications (Review). Oncol. Rep., 2016. 35(5): p. 2499-515.

17. Ojha, T., et al., Pharmacological and physical vessel modulation strategies to improve EPRmediated drug targeting to tumors. Adv. Drug Deliv. Rev., 2017. 119: p. 44-60.

18. Boissenot, T., et al., Ultrasound-triggered drug delivery for cancer treatment using drug delivery systems: From theoretical considerations to practical applications. J Control. Release, 2016. 241: p. 144-163.

19. Dicheva, B.M. and G.A. Koning, Targeted thermosensitive liposomes: an attractive novel approach for increased drug delivery to solid tumors. Expert Opin. Drug Deliv., 2014. 11(1): p. 83-100.

20. Facciorusso, A., Drug-eluting beads transarterial chemoembolization for hepatocellular carcinoma: Current state of the art., World J. Gastroenterol., 2018. 24(2): p. 161-169.

21. Fuchs, K., et al., Drug-eluting embolic microspheres for local drug delivery - State of the art., J Control. Release, 2017. 26: p. 127-138.

22. Wood, B.J., et al., Phase I Study of Heat-Deployed Liposomal Doxorubicin during Radiofrequency Ablation for Hepatic Malignancies. J. Vasc. Interv. Radiol., 2012. 23(2): p. 248-255. 
23. Tak, W.Y., et al., Phase III HEAT Study adding lyso-thermosensitive liposomal doxorubicin to radiofrequency ablation in patients with unresectable hepatocellular carcinoma lesions. Clin. Cancer Res., 2018. 24(1): p. 73-83.

24. Lyon, P.C., et al., Safety and feasibility of ultrasound-triggered targeted drug delivery of doxorubicin from thermosensitive liposomes in liver tumours (TARDOX): a single-center, openlabel, phase 1 trial. Lancet Oncol., 2018, 19(8): p. 1027-1039.

25. Woo, H.Y. and J. Heo, Transarterial chemoembolization using drug eluting beads for the treatment of hepatocellular carcinoma: now and future. Clin. Mol. Hepatol., 2015. 21(4): p. 344-348.

26. Pesapane, F., et al., New concepts in embolotherapy of HCC. Med. Oncol., 2017. 34(4): p.118.

27. Levy, J., et al., Intra-arterial therapies for unresectable and chemorefractory colorectal cancer liver metastases: a systematic review and meta-analysis. HPB (Oxford), 2018. 20(10): p. 905-915.

28. Young, S., et al., Review of the clinical evidence for the use of DEBIRI in the treatment of colorectal metastatic disease. Cardiovasc. Interv. Radiol., 2017. 40(4): p. 496-501.

29. Lee, E.W., S. Khan, and E.W. Lee, Recent advances in transarterial embolotherapies in the treatment of hepatocellular carcinoma. Clin. Mol. Hepatol., 2017. 23(4): p. 265-272.

30. Lencioni, R., et al., Treatment of intermediateladvanced hepatocellular carcinoma in the clinic: how can outcomes be improved? Oncologist, 2010. 15(Suppl. 4): p. 42-52.

31. Hong, C.W., S.K. Libutti, and B.J. Wood, Liposomal doxorubicin plus radiofrequency ablation for complete necrosis of a hepatocellular carcinoma. Curr. Oncol., 2013. 20(3): p. e274-7.

32. Shi, Y. and B. Zhai, A Recent Advance in Image-Guided Locoregional Therapy for Hepatocellular Carcinoma. Gastrointest. Tumors, 2016. 3(2): p. 90-102.

33. Ta, T. and T.M. Porter, Thermosensitive liposomes for localized delivery and triggered release of chemotherapy. J. Control. Release, 2013. 169(1-2): p. 112-125.

34. Swenson, C.E., et al., Increased duration of heating boosts local drug deposition during radiofrequency ablation in combination with thermally sensitive liposomes (ThermoDox) in a porcine model. PLoS One, 2015. 10(10): p. e0139752/1-e0139752/15.

35. Frich, L., et al., Experimental application of thermosensitive paramagnetic liposomes for monitoring magnetic resonance imaging guided thermal ablation. Magn. Reson. Med., 2004. 52(6): p. 1302-1309.

36. Andriyanov, A.V., et al., Therapeutic efficacy of combining pegylated liposomal doxorubicin and radiofrequency $(R F)$ ablation: comparison between slow-drug-releasing, non-thermosensitive and fast-drug-releasing, thermosensitive nano-liposomes. PLoS One, 2014. 9(5): p. e92555.

37. Dou, Y., K. Hynynen, and C. Allen,. To heat or not to heat: challenges with clinical translation of thermosensitive liposomes. J. Control. Release, 2017. 249: p. 63-73.

38. Crommelin, D.J.A., P. van Hoogevest, and G. Storm, The role of liposomes in clinical nanomedicine development. What now? Now what? J. Control. Release, 2020. 318: p. 256263.

39. Zheng, J., D. Jaffray, and C. Allen, Quantitative CT imaging of the spatial and temporal distribution of liposomes in a rabbit tumor model. Mol. Pharma., 2009. 6(2): p. 571-580.

40. Facciorusso, A., et al., Transarterial chemoembolization: evidences from the literature and applications in hepatocellular carcinoma patients. World J. Hepatolo., 2015. 7(16): p. 20092019.

41. Ma, J., et al., Intraarterial liver-directed therapies: the role of interventional oncology. Ochsner J., 2017. 17(4): p. 412-416. 
42. Piardi, T., et al., Management of large hepatocellular carcinoma by sequential transarterial chemoembolization and portal vein embolization: a systematic review of the literature. Minerva Chir, 2016. 71(3): p. 192-200.

43. Megerle, F., et al., Advanced adrenocortical carcinoma-what to do when first-line therapy fails? Exp. Clin. Endocrinol. Diabetes, 2019. 127(2-03): p. 109-116.

44. Freedman, R.A. and S.M. Tolaney, Efficacy and safety in older patient subsets in studies of endocrine monotherapy versus combination therapy in patients with HR+/HER2-advanced breast cancer: a review. Breast Cancer Res. Treat., 2018. 167(3): p. 607-614.

45. Maeda, H., Toward a full understanding of the EPR effect in primary and metastatic tumors as well as issues related to its heterogeneity. Adv. Drug. Deliv. Rev., 2015. 91: p. 3-6.

46. Prabhakar, U., et al., Challenges and key considerations of the enhanced permeability and retention effect for nanomedicine drug delivery in oncology. Cancer Res., 2013. 73(8): p. 24122417.

47. Yingchoncharoen, P., D.S. Kalinowski, and D.R. Richardson, Lipid-based drug delivery systems in cancer therapy: what is available and what is yet to come. Pharmacol. Rev., 2016. 68(3): p. 701-787.

48. Tay, C.Y., M.I. Setyawati, and D.T. Leong, Nanoparticle density: a critical biophysical regulator of endothelial permeability. ACS. Nano., 2017. 11(3): p. 2764-2772.

49. Setyawati, M.I., V.N. Mochalin, and D.T. Leong, Tuning endothelial permeability with functionalized nanodiamonds. ACS. Nano., 2016. 10(1): p. 1170-1181.

50. Wang, J.P., et al., Targeting endothelial cell junctions with negatively charged gold nanoparticles. Chem. Mater., 2018. 30(11): p. 3759-3767.

51. Setyawati, M.I., et al., Titanium dioxide nanomaterials cause endothelial cell leakiness by disrupting the homophilic interaction of VE-cadherin., Nat. Commun., 2013. 4: p. 1-12.

52. Daum, N., et al., Novel approaches for drug delivery systems in nanomedicine: effects of particle design and shape. Wiley Interdiscip. Rev. Nanomed. Nanobiotechnol., 2012. 4(1): p. 52-65.

53. Geng, Y., et al., Shape effects of filaments versus spherical particles in flow and drug delivery. Nat. Nanotechnol., 2007. 2(4): p. 249-255.

54. Mani, G., Stent-based drug delivery systems: current challenges and future trends. Ther. Deliv., 2013. 4(9): p. 1079-1082.

55. Decuzzi, P., et al., Intravascular delivery of particulate systems: does geometry really matter? Pharm. Res., 2009. 26(1): p. 235-243.

56. Allen, T. and P. Cullis, Drug delivery systems: entering the mainstream. Science, 2004. 303(5665): p. 1818-1822.

57. Duncan, R., The dawning era of polymer therapeutics. Nat. Rev. Drug Discov., 2003. 2(5): p. 347-360.

58. Jabir, N.R., et al., An overview on the current status of cancer nanomedicines. Curr. Med. Res. Opin., 2018. 34(5): p. 911-921.

59. Hu, J., et al., Advances in biomaterials and technologies for vascular embolization. Adv. Mater., 2019. 31(33): e1901071.

60. Doucet, J., et al., Advances in degradable embolic microspheres: a state of the art review. J. Funct. Biomater., 2018. 9(1): p. 14/1-14/24.

61. Lewis, A.L., et al., Doxorubicin eluting beads-1: Effects of drug loading on bead characteristics and drug distribution. J. Mate. Sci. Mater. Med., 2007. 18(9): p. 1691-1699.

62. Sharma, K.V., et al., Development of "Imageable" Beads for Transcatheter Embolotherapy. J. Vasc. Interv. Radiol., 2010. 21(6): p. 865-876. 
63. Grimaldi, N., et al., Lipid-based nanovesicles for nanomedicine. Chem. Soc. Rev., 2016. 45(23): p. 6520-6545.

64. Iyer, A.K., J. He, and M.M. Amiji, Image-guided nano-systems for targeted delivery in cancer therapy. Curr. Med. Chem., 2012. 19(19): p. 3230-3240.

65. Nicolaou, K.C. and S. Rigol, The role of organic synthesis in the emergence and development of antibody-drug conjugates as targeted cancer therapies. Angew. Chem., Int. Ed., 2019. 58 (33): p. 11206-11241.

66. Wang, A.Z., R. Langer, and O.C. Farokhzad, Nanoparticle delivery of cancer drugs. Annu. Rev. Med., 2012. 63: p. 185-198.

67. Zhu, L. and V.P. Torchilin, Stimulus-responsive nano-preparations for tumor targeting. Integr. Biol., 2013. 5(1): p. 96-107.

68. Alexis, F., et al., Nanoparticle technologies for cancer therapy. Handb. Exp. Pharmacol., 2010(197): p. 55-86.

69. Prull, C.-R., Part of a scientific master plan? Paul Ehrlich and the origins of his receptor concept. Med. Hist., 2003. 47(3): p. 332-56.

70. Rangachari, D. and J.R. Brahmer, Targeting the immune system in the treatment of non-smallcell lung cancer. Curr. Treat. Options. Oncol., 2013. 14(4): p. 580-94.

71. Fernandes, C., D. Suares, and M. Yergeri, Tumor microenvironment targeted nano-therapy. Front. Pharmacol., 2018. 9: p. 1230.

72. Torchilin, V.P., Passive and active drug targeting: drug delivery to tumors as an example. Handb. Exp. Pharmacol., 2010(197): p. 3-53.

73. Maeda, H., T. Sawa, and T. Konno, Mechanism of tumor-targeted delivery of macromolecular drugs, including the EPR effect in solid tumor and clinical overview of the prototype polymeric drug SMANCS. J. Control. Release., 2001. 74(1-3): p. 47-61.

74. Kim, J.H., I. Park, and J.L. Lee, Pazopanib versus sunitinib for the treatment of metastatic renal cell carcinoma patients with poor-risk features. Cancer Chemother. Pharmacol., 2016. 78(2): p. 325-32.

75. Maeda, H., H. Nakamura, and J. Fang, The EPR effect for macromolecular drug delivery to solid tumors: improvement of tumor uptake, lowering of systemic toxicity, and distinct tumor imaging in vivo. Adv. Drug Deliv. Rev., 2013. 65(1): p. 71-79.

76. Hare, J.I., et al., Challenges and strategies in anti-cancer nanomedicine development: an industry perspective. Adv. Drug Deliv. Rev., 2017. 108: p. 25-38.

77. Hashizume, H., et al., Openings between defective endothelial cells explain tumor vessel leakiness. Am. J. Pathol., 2000. 156(4): p. 1363-80.

78. Jain, R.K. and T. Stylianopoulos, Delivering nanomedicine to solid tumors. Nat. Rev. Clin. Oncol., 2010. 7(11): p. 653-64.

79. Allen, T.M. and C. Hansen, Pharmacokinetics of stealth versus conventional liposomes effect of dose. Biochim. Biophys. Acta, 1991. 1068(2): p. 133-141.

80. Ponce, A.M., et al., Hyperthermia mediated liposomal drug delivery. Int. J. Hyperth., 2006. 22(3): p. 205-213.

81. Bobo, D., et al., Nanoparticle-based medicines: a review of FDA-approved materials and clinical trials to date. Pharm. Res., 2016. 33(10): p. 2373-2387.

82. Havel, H., et al., Nanomedicines: from bench to bedside and beyond. AAPS J., 2016. 18(6): p. 1373-1378.

83. Anderson, P.M., M. Tomaras, and K. McConnell, Mifamurtide in osteosarcoma-a practical review. Drugs of Today, 2010. 46(5): p. 327-337. 
84. Ando, K., et al., Mifamurtide for the treatment of nonmetastatic osteosarcoma. Expert Opin. Pharmacother., 2011. 12(2): p. 285-292.

85. Bulbake, U., et al., Liposomal formulations in clinical use: an updated review. Pharmaceutics, 2017. 9(2): p.12-45.

86. Lamichhane, N., et al., Liposomes: clinical applications and potential for image-guided drug delivery. Molecules, 2018. 23(2): p. 288/1-288/17.

87. Zhu, L., L. Yang, and Z.Y. Zhou, Nanomaterials in cancer theranostics. Bioactivity of engineered nanoparticles, 2017: p. 173-206.

88. Ahmad, A., et al., Precision cancer nano-therapy: evolving role of multifunctional nanoparticles for cancer active targeting. J. Med. Chem., 2019. 62(23): p. 10475-10496

89. Torchilin, V.P., Multifunctional, stimuli-sensitive nanoparticulate systems for drug delivery. Nat. Rev. Drug Discovery., 2014. 13(11): p. 813-827.

90. Merten, H., et al., Antibody-drug conjugates for tumor targeting-novel conjugation chemistries and the promise of non-IgG binding proteins. Bioconjugate Chem., 2015. 26(11): p. 21762185.

91. Garde, S.V., et al., Binding and internalization of NGR-peptide-targeted liposomal doxorubicin (TVT-DOX) in CD13-expressing cells and its antitumor effects. Anti-Cancer Drugs, 2007. 18(10): p. 1189-1200.

92. Accardo, A. and G. Morelli, Review peptide-targeted liposomes for selective drug delivery: advantages and problematic issues. Biopolymers, 2015. 104(5): p. 462-479.

93. Deng, Y., J. Ling, and M.-H. Li, Physical stimuli-responsive liposomes and polymersomes as drug delivery vehicles based on phase transitions in the membrane. Nanoscale, 2018. 10(15): p. 6781-6800.

94. Anajafi, T. and S. Mallik, Polymersome-based drug delivery strategies for cancer therapeutics. Ther. Deliv., 2015. 6(4): p. 521-534.

95. Ganta, S., et al., A review of stimuli-responsive nanocarriers for drug and gene delivery. J. Control. Release., 2008. 126(3): p. 187-204.

96. Mura, S., J. Nicolas, and P. Couvreur, Stimuli-responsive nanocarriers for drug delivery. Nat. Mater., 2013. 12(11): p. 991-1003.

97. May, J.P. and S.-D. Li, Thermosensitive liposomes in cancer therapy. Recent Pat. Biomed. Eng., 2012. 5(2): p. 148-158.

98. Kneidl, B., et al., Thermosensitive liposomal drug delivery systems: state of the art review. Int. J. Nanomed., 2014. 9: p. 4387-4398.

99. Al-Ahmady, Z. and K. Kostarelos, Chemical components for the design of temperature-responsive vesicles as cancer therapeutics. Chem. Rev. (Washington, DC, U. S.), 2016. 116(6): p. 38833918.

100. Ho, L., M. Bokharaei, and S.-D. Li, Current update of a thermosensitive liposomes composed of DPPC and Brij78. J. Drug Targeting, 2018. 26(5-6): p. 407-419.

101. Rabiskova, M., E. Koziolova, and J. Jiraskova, Nanoparticulates with drug release based on temperature change. Ceska Slov. Farm., 2014. 63(6): p. 239-247.

102. Kong, G., et al., Efficacy of liposomes and hyperthermia in a human tumor xenograft model: importance of triggered drug release. Cancer Res., 2000. 60(24): p. 6950-6957.

103. Needham, D., et al., A new temperature-sensitive liposome for use with mild hyperthermia: characterization and testing in a human tumor xenograft model. Cancer Res., 2000. 60(5): p. 1197-1201. 
104. Ranjan, A., et al., Image-guided drug delivery with magnetic resonance guided high intensity focused ultrasound and temperature sensitive liposomes in a rabbit Vx2 tumor model. J. Control. Release., 2012. 158(3): p. 487-494.

105. Staruch, R.M., et al., Enhanced drug delivery in rabbit VX2 tumours using thermosensitive liposomes and MRI-controlled focused ultrasound hyperthermia. Int. J. Hyperth., 2012. 28(8): p. 776-787.

106. Santos, M.A., D.E. Goertz, and K. Hynynen, Focused ultrasound hyperthermia mediated drug delivery using thermosensitive liposomes and visualized with in vivo two-photon microscopy. Theranostics, 2017. 7(10): p. 2718-2731.

107. Bing, C., et al., Longer heating duration increases localized doxorubicin deposition and therapeutic index in Vx2 tumors using MR-HIFU mild hyperthermia and thermosensitive liposomal doxorubicin. Int. J. Hyperth., 2019. 36(1): p. 196-203.

108. Majekodunmi, S.O., Review of current trends in enhancing bioavailability of poorly water soluble drugs by liposomal interventions. J. Pharm., 2016. 6(8-1): p. 30-39.

109. Arora, J.S., et al., Ablative focused ultrasound synergistically enhances thermally triggered chemotherapy for prostate cancer in vitro. Mol. Pharm., 2016. 13(9): p. 3080-3090.

110. Staruch, R.M., K. Hynynen, and R. Chopra, Hyperthermia-mediated doxorubicin release from thermosensitive liposomes using MR-HIFU: therapeutic effect in rabbit Vx2 tumours. Int. J. Hyperth., 2015. 31(2): p. 118-133.

111. Lindstrom, P.A., Prefrontal ultrasonic irradiation-a substitute for lobotomy. Arch. Neurol. Psychiatry, 1954. 72(4): p. 399-425.

112. Babalola, O., T.H.J. Lee, and C.J. Viviano, Prostate ablation using high intensity focused ultrasound: a literature review of the potential role for patient preference information. J. Urol., 2018. 200(3): p. 512-519.

113. Ringold, S., FDA approves ultrasound fibroid therapy. J. Am. Med. Assoc., 2004. 292(23): p. 2826.

114. Scipione, R., et al., HIFU for bone metastases and other musculoskeletal applications. Semin. Interv. Radiol., 2018. 35(4): p. 261-267.

115. Ranjan, M., et al., Neuromodulation beyond neurostimulation for epilepsy: scope for focused ultrasound. Expert Rev. Neurother., 2019. 19 (10): p. 937-943.

116. Hijnen, N., S. Langereis, and H. Grüll, Magnetic resonance guided high-intensity focused ultrasound for image-guided temperature-induced drug delivery. Adv. Drug Deliv. Rev., 2014. 72: p. 65-81.

117. Hynynen, K., MRIgHIFU: a tool for image-guided therapeutics. J. Magn. Reson. Imaging, 2011. 34(3): p. 482-93.

118. Gruell, H. and S. Langereis, Hyperthermia-triggered drug delivery from temperature-sensitive liposomes using MRI-guided high intensity focused ultrasound. J. Control. Release, 2012. 161(2): p. 317-327.

119. McDannold, N., et al., MRI evaluation of thermal ablation of tumors with focused ultrasound. J. Magn. Reson. Imaging, 1998. 8(1): p. 91-100.

120. Rieke, V. and K.B. Pauly, MR thermometry. J. Magn. Reson. Imaging, 2008. 27(2): p. 376390.

121. Gray, M.D., et al., Focused ultrasound hyperthermia for targeted drug release from thermosensitive liposomes: results from a phase I trial. Radiology, 2019. 291(1): p. 232-238.

122. Lyon, P.C., et al., Clinical trial protocol for TARDOX: a phase I study to investigate the feasibility of targeted release of lyso-thermosensitive liposomal doxorubicin (ThermoDox ${ }^{\odot}$ ) using focused ultrasound in patients with liver tumours. J. Ther. Ultrasound, 2017. 5: p. 28. 
123. Nardecchia, S., et al., Clinical trials of thermosensitive nanomaterials: an overview. Nanomaterials (Basel), 2019.9 (2): p. 1-23.

124. Celik, H., et al., Radiofrequency ablation duration per tumor volume may correlate with overall survival in solitary hepatocellular carcinoma patients treated with radiofrequency ablation plus lyso-thermosensitive liposomal doxorubicin. J. Vasc. Interv. Radiol., 2019. 30(12): p. 19081914.

125. Lyon, P.C., et al., Safety and feasibility of ultrasound-triggered targeted drug delivery of doxorubicin from thermosensitive liposomes in liver tumours (TARDOX): a single-center, openlabel, phase 1 trial. Lancet Oncol., 2018. 19(8): p. 1027-1039.

126. Pastorino, F., et al., Targeting liposomal chemotherapy via both tumor cell-specific and tumor vasculature-specific ligands potentiates therapeutic efficacy. Cancer Res., 2006. 66(20): p. 10073-10082.

127. Neri, D. and R. Bicknell, Tumour vascular targeting. Nat. Rev. Cancer, 2005. 5(6): p. 436446.

128. Koren, E. and V.P. Torchilin, Drug carriers for vascular drug delivery. Iubmb Life, 2011. 63(8): p. 586-595.

129. Hashimoto, Y., et al., Coating of liposomes with subunits of monoclonal IgM antibody and targeting of the liposomes, in Methods Enzymol. 1986, Academic Press. p. 817-828.

130. Kamaly, N., et al., Folate receptor targeted bimodal liposomes for tumor magnetic resonance imaging. Bioconjugate Chem., 2009. 20(4): p. 648-655.

131. Xu, S., et al., Targeting receptor-mediated endocytotic pathways with nanoparticles: rationale and advances. Adv. Drug Deliv. Rev., 2013. 65(1): p. 121-138.

132. Daniels, T.R., et al., The transferrin receptor and the targeted delivery of therapeutic agents against cancer. Biochim. Biophys. Acta (BBA)-Gen. Subj., 2012. 1820(3): p. 291-317.

133. Daniels, T.R., et al., The transferrin receptor part I: biology and targeting with cytotoxic antibodies for the treatment of cancer. Clin. Immunol., 2006. 121(2): p. 144-158.

134. Gomme, P.T., K.B. McCann, and J. Bertolini, Transferrin: structure, function and potential therapeutic actions. Drug Discov. Today, 2005. 10(4): p. 267-273.

135. Cho, K., et al., Therapeutic nanoparticles for drug delivery in cancer. Clin. Cancer Res., 2008. 14(5): p. 1310-6.

136. Low, P.S. and S.A. Kularatne, Folate-targeted therapeutic and imaging agents for cancer. Curr. Opin. Chem. Biol., 2009. 13(3): p. 256-62.

137. Parker, N., et al., Folate receptor expression in carcinomas and normal tissues determined by a quantitative radioligand binding assay. Anal. Biochem., 2005. 338(2): p. 284-293.

138. Sudimack, J. and R.J. Lee, Targeted drug delivery via the folate receptor. Adv. Drug Deliv. Rev., 2000. 41(2): p. 147-162.

139. Minko, T., Drug targeting to the colon with lectins and neoglycoconjugates. Adv. Drug Deliv. Rev., 2004. 56(4): p. 491-509.

140. Acharya, S., F. Dilnawaz, and S.K. Sahoo, Targeted epidermal growth factor receptor nanoparticle bioconjugates for breast cancer therapy. Biomaterials, 2009. 30(29): p. 5737-50.

141. Scaltriti, M. and J. Baselga, The epidermal growth factor receptor pathway: a model for targeted therapy. Clin. Cancer Res., 2006. 12(18): p. 5268-72.

142. Lurje, G. and H.J. Lenz, EGFR signaling and drug discovery. Oncology, 2009. 77(6): p. 400-10.

143. Shadidi, M. and M. Sioud, Selective targeting of cancer cells using synthetic peptides. Drug Resist. Updat., 2003. 6(6): p. 363-71. 
144. Carmeliet, P., VEGF as a key mediator of angiogenesis in cancer. Oncology, 2005. 69 Suppl 3: p. 4-10.

145. Desgrosellier, J.S. and D.A. Cheresh, Integrins in cancer: biological implications and therapeutic opportunities. Nat. Rev. Cancer, 2010. 10(1): p. 9-22.

146. Saiki, I., et al., Role of aminopeptidase N (CD13) in tumor-cell invasion and extracellular matrix degradation. Int. J. Cancer, 1993. 54(1): p. 137-43.

147. Pasqualini, R., et al., Aminopeptidase $N$ is a receptor for tumor-homing peptides and a target for inhibiting angiogenesis. Cancer Res., 2000. 60(3): p. 722-727.

148. Arap, W., R. Pasqualini, and E. Ruoslahti, Cancer treatment by targeted drug delivery to tumor vasculature in a mouse model. Science, 1998. 279(5349): p. 377-380.

149. Belfiore, L., et al., Towards clinical translation of ligand-functionalized liposomes in targeted cancer therapy: challenges and opportunities. J. Control. Release, 2018. 277: p. 1-13.

150. Lale, S.V., et al., AS1411 aptamer and folic acid functionalized $p H$-responsive ATRP fabricated pPEGMA-PCL-pPEGMA polymeric nanoparticles for targeted drug delivery in cancer therapy. Biomacromolecules, 2014. 15(5): p. 1737-1752.

151. Lukyanov, A.N., et al., Tumor-targeted liposomes: doxorubicin-loaded long-circulating liposomes modified with anti-cancer antibody. J. Control. Release, 2004. 100(1): p. 135-144.

152. Sapra, P. and T.M. Allen, Improved outcome when B-cell lymphoma is treated with combinations of immunoliposomal anticancer drugs targeted to both the CD19 and CD20 epitopes. Clin. Cancer Res., 2004. 10(7): p. 2530-2537.

153. Avula, M.N. and D.W. Grainger. Addressing medical device challenges with drug-device combinations. 2015. John Wiley \& Sons, Inc.

154. Poursaid, A., et al., Polymeric materials for embolic and chemoembolic applications. J. Control. Release, 2016. 240: p. 414-433.

155. Gallicchio, R., et al., Therapeutic strategies in HCC: radiation modalities. BioMed. Res. Int., 2016. p. 1295329/1-1295329/11.

156. Toskich, B., Locoregional therapies for hepatocellular carcinoma. Precision Molecular Pathology of Liver Cancer, ed. C. Liu. 2018, Switzerland: Springer International Publishing AG. 213233.

157. Yang, K., et al., Advanced imaging techniques in the therapeutic response of transarterial chemoembolization for hepatocellular carcinoma. World J. Gastroenterol., 2016. 22(20): p. 4835-4847.

158. Zhang, X.-P., et al., Survival benefit of hepatic resection versus transarterial chemoembolization for hepatocellular carcinoma with portal vein tumor thrombus: a systematic review and metaanalysis. BMC Cancer, 2017. 17: p. 902/1-902/14.

159. Vogl, T.J., et al., Review on transarterial chemoembolization in hepatocellular carcinoma: Palliative, combined, neoadjuvant, bridging, and symptomatic indications. Eur. J. Radiol., 2009. 72(3): p. 505-516.

160. Budker, V.G., S.D. Monahan, and V.M. Subbotin, Loco-regional cancer drug therapy: present approaches and rapidly reversible hydrophobization (RRH) of therapeutic agents as the future direction. Drug Discov. Today, 2014. 19(12): p. 1855-1870.

161. Klopp, C.T., Regional intra-arterial nitrogen mustard as an adjunct to radiation therapy. Am. J. Roentgenol. Radium. Ther. Nucl. Med., 1953. 70(6): p. 1005-14.

162. Klopp, C.T., et al., Fractionated intra-arterial cancer; chemotherapy with methyl bis amine hydrochloride; a preliminary report. Ann. Surg., 1950. 132(4): p. 811-32.

163. Tyler, J.L., et al., Pharmacokinetics of super elective intraarterial and intravenous C-11 BCNU evaluated by PET. J. Nucl. Med., 1986. 27(6): p. 775-780. 
164. Dreher, M.R., et al., Radiopaque drug-eluting beads for transcatheter embolotherapy: experimental study of drug penetration and coverage in swine. J. Vasc. Interv. Radiol., 2012. 23(2): p. 257-264.

165. Habib, A., et al., Transarterial approaches to primary and secondary hepatic malignancies. Nat. Rev. Clin. Oncol., 2015. 12(8): p. 481-9.

166. Nijsen, F., et al., Targeting of liver tumour in rats by selective delivery of Holmium-166 loaded microspheres: a biodistribution study. Eur. J. Nucl. Med., 2001. 28(6): p. 743-749.

167. Johnson, C.G., et al., Preparation of radiopaque drug-eluting beads for transcatheter chemoembolization. J. Vasc. Interv. Radiol., 2015. 27 (1): p. 117-126.

168. He, P., et al., Material discrimination based on k-edge characteristics. Comput. Math. Methods Med., 2013. 2013: p. 308520.

169. Mikhail, A.S., et al., Mapping drug dose distribution on CT images following transarterial chemoembolization with radiopaque drug-eluting beads in a rabbit tumor model. Radiology, 2018. 289(2): p. 396-404.

170 Pritchard, W.F., et al. Hepatic arterial embolization with radiopaque microbeads containing iodine or bismuth in swine: high resolution imaging and material decomposition with photoncounting CT. in Workshop on Medical Applications of Spectroscopic X-ray Detectors. 2019. Switzerland: CERN.

171. Levy, E.B., et al., First human experience with directly image-able iodinated embolization microbeads. Cardiovasc. Interv. Radiol., 2016. 39(8): p. 1177-1186.

172 Ishida, T., et al., Accelerated clearance of PEGylated liposomes in rats after repeated injections. J. Control. Release, 2003. 88(1): p. 35-42.

173 Torchilin, V., Tumor delivery of macromolecular drugs based on the EPR effect. Adv. Drug Deliv. Rev., 2011. 63(3): p. 131-135. 


\section{Chapter 2}

\section{Formulation and characterization of magnetic resonance image-able thermally sensitive liposomes for use with magnetic resonance-guided high intensity focused ultrasound}

Ayele H. Negussie*1, Pavel S. Yarmolenko*1,2, Ari Partanen ${ }^{1,3}$, Ashish Ranjan ${ }^{1}$, Genevive Jacobs ${ }^{1}$, David Woods ${ }^{1}$, Henry Bryant ${ }^{4}$, David Thomasson ${ }^{5}$, Mark W. Dewhirst $^{6}$, Bradford J. Wood ${ }^{1}$, Matthew R. Dreher ${ }^{1}$

${ }^{1}$ Center for Interventional Oncology, Radiology and Imaging Sciences, Clinical Center, National Institutes of Health, Bethesda, Maryland

${ }^{2}$ Department of Biomedical Engineering, Duke University, Durham, North Carolina

${ }^{3}$ Philips Healthcare, Cleveland, Ohio

${ }^{4}$ Laboratory of Diagnostic Radiology Research, Radiology and Imaging Sciences, Clinical Center, National Institutes of Health, Bethesda, Maryland

${ }^{5}$ Radiology and Imaging Sciences, Clinical Center, National Institutes of Health, Bethesda, Maryland, and ${ }^{6}$ Department of Radiation Oncology, Duke University, Durham, North Carolina, USA.

Published in: Int. J. Hyperther., 27: 2011

*Authors contributed equally 


\section{Abstract}

Conventional anticancer chemotherapeutic agents demonstrate limited specificity for tumor tissue that often results in dose-limiting toxicity and reduced therapeutic efficacy. Therefore, development of drug delivery systems (DDSs) that may selectively deliver anticancer drugs to a tumor with less toxic side effects with wide therapeutic window is important. The goal of this study were, 1) develop iLTSL, a low temperature sensitive liposome co-loaded with an MRI contrast agent (ProHance ${ }^{\oplus}$ Gd-HP-DO3A) and doxorubicin, 2) characterize doxorubicin and Gd-HP-DO3A release from iLTSL and 3) investigate the ability of magnetic resonance-guided high intensity focused ultrasound (MR-HIFU) to induce and monitor iLTSL content release in phantoms and in vivo. These were achieved by preparing iLTSL passively loaded with Gd-HP-DO3A and actively loaded with doxorubicin. Doxorubicin and Gd-HP-DO3A release was quantified by fluorescence and spectroscopic techniques, respectively. Release with MR-HIFU was examined in tissue-mimicking phantoms containing iLTSL and in a VX2 rabbit tumor model. The resulting iLTSL demonstrated consistent size and doxorubicin release kinetics after storage at $4^{\circ} \mathrm{C}$ for 7 days. Release of doxorubicin and Gd-HP-DO3A from iLTSL was minimal at $37^{\circ} \mathrm{C}$ but fast when heated to $41.3{ }^{\circ} \mathrm{C}$. The magnitude of release was not significantly different between doxorubicin and Gd-HP-DO3A over $10 \mathrm{~min}$ in HEPES buffer and plasma at $37^{\circ}, 40^{\circ}$ and $41.3{ }^{\circ} \mathrm{C}(p>0.05)$. Relaxivity of iLTSL increased significantly ( $p$ $<0.0001$ ) from $1.95 \pm 0.05$ to $4.01 \pm 0.1 \mathrm{mMs}^{-1}$ when heated above the transition temperature. Signal increase corresponded spatially and temporally to MR-HIFUheated locations in phantoms. Signal increase was also observed in vivo after iLTSL injection and after each 10 -min heating $\left(41^{\circ} \mathrm{C}\right)$, with greatest increase in the heated tumor region. 
An MR image-able liposome formulation co-loaded with doxorubicin and an MR contrast agent was developed. Stability, image-ability, and MR-HIFU monitoring and control of content release suggest that MR-HIFU combined with iLTSL may enable real-time monitoring and spatial control of content release.

\section{Introduction}

Conventional anticancer chemotherapeutic agents demonstrate limited specificity for tumor tissue that often results in dose-limiting toxicity and reduced therapeutic efficacy. Therefore, development of drug delivery systems (DDS) that may selectively deliver anticancer drugs to a tumor with less toxic side effects with wide therapeutic window is important [1]. Among different DDSs, liposomes have a long history of delivering both therapeutic and diagnostic agents thereby, resulting in a number of liposomal agents used in clinics $[1,2]$.

Liposomal DDSs target a solid tumor either 'passively', because of tailorable particle size and long circulating capability, or 'actively', due to a specific affinity or activation by stimuli. An example of passive targeting is selective accumulation of stealth (PEGylated) liposomes in solid tumors by a mechanism known as enhanced permeability and retention (EPR) effect [3, 4]. This approach has often resulted in 10-fold or greater drug delivery to a tumor over conventional chemotherapy [5]. Active or stimuli responsive drug delivery can be achieved through incorporation of tumor-specific targeting ligands on the surface of the liposome $[6,7]$ as well as liposomal components sensitive to various stimuli such as $\mathrm{pH}[8,9]$, electromagnetic radiation [10, 11], enzymes [12, 13] and temperature [14-16]. 
Liposomal formulations with drug release properties that are triggered by heat are called temperature sensitive liposomes (TSLs) [14]. Incorporation of lysolipids in TSLs formulations resulted in rapid release of liposomal contents when heated to mild hyperthermia ( $>40{ }^{\circ} \mathrm{C}$ but $<45^{\circ} \mathrm{C}$ ), slightly lower maximum release temperature than the classical TSLs [15]. These liposomes, with release properties optimized for mild hyperthermia, are often called low temperature sensitive liposomes (LTSLs). Though material properties and efficacy of LTSLs in preclinical and clinical studies have been extensively studied [15, 17-22], precise spatiotemporal control of drug delivery with LTSL has not yet been achieved as this requires suitable hyperthermia applicator whose temperature outcome can precisely be predicted. As hyperthermia applicator, Microwave, radiofrequency and focused ultrasound have been used. Of these, focused ultrasound applicator is noninvasive and provides energy to treat tissue deep in the body precisely [23].

Further improvement in spatiotemporal control of drug delivery with LTSL may now be achieved with the recent advances in MR-guided heating methods, such as MR-guided high intensity focused ultrasound (MR-HIFU). MR-guided heating methods are especially relevant to drug delivery with LTSL since they enable to identify and target tissue to be treated, real time guidance and control and confirm the effectiveness of the treatment [24]. Such systems have been approved by the Food and Drug Administration (FDA) [25] and were in clinical trials for the treatment of uterine fibroids [26-31] with temperature feedback [32]. However, intra- and inter-patient spatial variability in tumor microenvironment, such as vascularity and perfusion, may impact both the heating pattern and delivery of drugs $[33,34]$. Therefore, in addition to temperature, direct imaging of drug delivery (or an appropriate surrogate, such as release of an MR contrast agent from LTSL) may improve real-time control of drug delivery or help guide localization of therapeutic delivery (such as local thermal ablation or local drug delivery). 
Co-loading of drug and MR contrast agent into LTSL may provide an appropriate surrogate for estimating drug delivered. The first liposomes loaded with MRI contrast agents appeared in the literature in the 1980s [35]. These liposomes encapsulated high concentrations $(670 \mathrm{mM})$ of hydrophilic contrast agents such as Gd-DTPA. Since then, similar Gd-based contrast agents have been incorporated into the liposome interior compartment [36] as well as conjugated to their membrane [37, 38] or both [39]. Most liposomes with Gd-based contrast agents in their interior compartment have a relaxivity lower than that of free contrast agent ( $>6$-fold lower for some liposomes at $1.5 \mathrm{~T}$ [35], because their liposomal membrane shields contrast agent in the interior compartment from bulk water outside the liposome [40]. Liposomes with contrast agents attached to their surface offer exceptionally high relaxivity ( $>3$-fold higher than free contrast agent at 1.5 $\mathrm{T}$ [38], likely due to high rotational correlation times of surface-bound contrast agents [37]. However, such liposomes with contrast agents only on the surface are not designed to report on drug release since there is no changes in relaxivity before and after hyperthermia treatment.

LTSLs that release contrast agent from their interior have been used to image release of liposomal contents in vivo. Such liposomes have demonstrated correlations of MR imaging with ablation margins [41], drug delivery [42] and therapeutic efficacy [43]. Even though both manganese and gadolinium-based contrast agents have been used to report on drug delivery with liposomes [42, 43], gadolinium-based contrast agents are more attractive due to their safety and the history of widespread use in clinics. Therefore, much of the recent work on MR image-able liposomes involves gadolinium-based contrast agents [44].

Ability to image and control content release from liposomes with MR-HIFU may allow for drug dose painting [42, 45]. Our dose painting approach employs 
MR-HIFU to induce drug and contrast agent release from image-able LTSLs, using both imaging of temperature changes and contrast agent release in real-time to control the HIFU transducer to achieve the desired drug distribution in the target volume. As an initial step in this image-guided dose painting concept, the objectives of this study were to: 1) develop iLTSL, an LTSL co-loaded with an FDA-approved MRI contrast agent (ProHance ${ }^{\oplus}$ Gd-HP-DO3A) and doxorubicin (Dox), 2) characterize release of drug and Gd- HP-DO3A from iLTSL, and 3) investigate the ability of MR-HIFU to induce and monitor iLTSL content release in phantoms and in vivo.

\section{Materials and methods}

\section{Materials}

ProHance $^{\oplus}$ (Gd-HP-DO3A, 500 mM) (Bracco Diagnostics, Princeton, NJ) was used as the MR contrast agent. Monostearoyl-2-hydroxy-sn-glycero-3phosphocholine (MSPC), 1,2- dipalmitoyl-sn-glycero-3-phosphocholine (DPPC), and 1,2-distearoyl-sn-glycero-3- phosphoethanolamine-N-[methoxy (Polyethylene Glycol)2000] (DSPE-PEG2000) were obtained from Genzyme Corporation (Cambridge, MA). Dox was obtained from Bedford laboratory (Bedford, $\mathrm{OH}$ ). High and low melting point agarose was purchased from Invitrogen (Carlsbad, CA) and ISC BioExpress (Kaysville, UT), respectively. All of the other chemicals were bought from Sigma-Aldrich (St Louis, MO).

\section{Preparation and characterizations of iLTSL liposomes}

Liposomes were prepared by hydration of lipid film, followed by extrusion as previously reported [46]. Briefly, lipid components (DPPC, MSPC and DSPEPEG2000) were dissolved in chloroform at a molar ratio of 85.3: 9.7: 5.0. The solvent was evaporated using a Rotovap system and left overnight in a vacuum 
desiccator. The resulting lipid film was hydrated by a buffer consisting of 300 mM Gd-HP-DO3A and 100 mM Citrate (520 \pm 12 mOsm [501-533 mOsm], $\mathrm{pH} 4.0$ ) at $60^{\circ} \mathrm{C}$ for $15 \mathrm{~min}$ to yield a final lipid concentration of $50 \mathrm{mg} / \mathrm{mL}$. Liposomes were obtained by extruding the mixture five times with a LIPEX ${ }^{\mathrm{TM}}$ extruder (Northern Lipids, Burnaby, BC) at $55^{\circ} \mathrm{C}$ through two stacked Nuclepore polycarbonate membrane filters (Whatman PLC, Maidstone, Kent, UK) with a pore size of $100 \mathrm{~nm}$.

Encapsulation of Dox into the extruded liposomes was carried out using a $\mathrm{pH}$-gradient loading protocol as described by Mayer et al. [46] with a slight modification: exterior $\mathrm{pH}$ of the extruded liposomes was adjusted to 7.4 with sodium carbonate solution $(500 \mathrm{mM})$ creating a $\mathrm{pH}$ gradient (acidic inside LTSL). The liposomes were incubated with Dox (Dox: lipid weight ratio of 5: 100) at $37^{\circ} \mathrm{C}$ for $1 \mathrm{~h}$. Un-encapsulated Gd-HP-DO3A and Dox were removed by passing the liposome through a Sephadex-G50 (fine) spin column and the resulting liposomes were stored at $4^{\circ} \mathrm{C}$ until further use.

Particle size of liposomes was determined by dynamic light scattering (Zetasizer Nano-ZS, Malvern Instruments, Westborough, MA) and reported as the mean Z-averaged diameter, and polydispersity index from a cumulants analysis of three measurements. The concentration of liposome-entrapped Dox was determined using a UV-Vis spectrophotometer (PerkinElmer, Waltham, MA) as previously reported [43].

\section{Dox release}

Release of encapsulated Dox from iLTSL as a function of time (up to $15 \mathrm{~min}$ ) and temperature $\left(25^{\circ}\right.$, and $\left.37^{\circ}-41.3^{\circ} \mathrm{C}\right)$ was assessed by measuring de-quenching of Dox fluorescence as Dox was released from the liposome. Two instruments were 
used to measure Dox fluorescence in release assays, as necessitated by experimental design. In experiments with high temporal resolution (ThermoScan and Dox release kinetics measurements), fluorescence was measured with a Cary Eclipse Spectro-fluorimeter equipped with an Eclipse multicell Peltier temperature controller, and Eclipse Kinetic and ThermoScan Software (Varian, Palo Alto, CA) at excitation and emission wavelengths of 498 and $593 \mathrm{~nm}$, respectively. In experiments where Dox and Gd-HP-DO3A release were both quantified, a Victor II plate reader was used (ex/em $=490 / 572 \mathrm{~nm}$, Perkin Elmer). Dox release was quantified in three different release assays:

1. ThermoScan release assay: In these experiments, dox release was assessed as a function of both temperature and time. iLTSL stock solution was added to $2 \mathrm{~mL}$ of either $10 \mathrm{mM}$ HEPES ( $\mathrm{pH} 7.4,140 \mathrm{mM} \mathrm{NaCl}, 280 \mathrm{mOsm}$ ) or human plasma (collected with apheresis, whole blood treated with 1: 12 ACD (anticoagulant citrate dextrose solution A)) in a quartz cuvette. The volumes of stock liposome solution added were 8 and $30 \mu \mathrm{L}$ in experiments for release in HEPES and plasma, respectively. The cuvette was then heated at a rate of $1^{\circ} \mathrm{C} / \mathrm{min}$ from $20^{\circ} \mathrm{C}$ to $55^{\circ} \mathrm{C}$ while stirring. Fluorescence readings were taken every 30 s. Another cuvette holder in the Peltier unit was used to monitor temperature. Differences in temperature among the three cuvette holders were less than $0.1{ }^{\circ} \mathrm{C}$ (data not shown). Data are presented as mean fluorescence intensity $(n=3)$.

2. Dox kinetic release assay: These experiments were designed to measure Dox release as a function of time at a constant temperature. The same volumes as above were used, but in this instance, buffer or plasma was first equilibrated to the desired temperature $\left(25^{\circ}, 37^{\circ}-41.3{ }^{\circ} \mathrm{C}\right)$. The temperature was maintained, and fluorescence intensity was measured every $7 \mathrm{~s}$ for $15 \mathrm{~min}$ while the sample was stirred. Percent release was calculated by assuming 100\% release with Triton ${ }^{\odot}$ 
$\mathrm{X}-100$ and $0 \%$ release at $25^{\circ} \mathrm{C}$. In HEPES, addition of Triton ${ }^{\oplus} \mathrm{X}-100$ resulted in a decrease $(6.7 \pm 0.3 \%, n=6)$ of Dox fluorescence intensity from the maximum obtained by heating at and above $41{ }^{\circ} \mathrm{C}$, and therefore the level of fluorescence used as $100 \%$ release was increased proportionately for all release assays of this type in HEPES. In plasma, addition of Triton ${ }^{\circledR} \mathrm{X}-100$ results in an increase of Dox fluorescence of $25.3 \%$ (same concentrations of Dox and Triton ${ }^{\circledR} \mathrm{X}-100$ as in the release study). The fluorescence intensity at $100 \%$ release (with Triton ${ }^{\circledR} \mathrm{X}-100$ ) was therefore adjusted proportionately. Data are presented as mean percentage release of three samples $(n=3)$.

3. Simultaneous Dox and Gd-HP-DO3A release assay: HEPES buffer (14 mL) or human plasma $(7 \mathrm{~mL})$ were allowed to equilibrate in a round-bottomed flask (at $37^{\circ}, 40^{\circ}$, or $\left.41.3^{\circ} \mathrm{C}\right)$. Liposome stock solution was added ( $1 \mathrm{~mL}$ added to HEPES and $0.5 \mathrm{~mL}$ to plasma), while mixing with a magnetic stir bar. Aliquots $(0.25$ $\mathrm{mL}$ in HEPES release assay and $0.2 \mathrm{~mL}$ in plasma release assay) were withdrawn at predetermined time points. To capture the instantaneous release at each time point, each aliquot was added to $0.75 \mathrm{~mL}$ of cooled HEPES buffer or $0.4 \mathrm{~mL}$ human plasma on ice. Samples were kept on ice, and longitudinal relaxation rate $\left(\mathrm{R}_{1}=1 / \mathrm{T}_{1}\right)$ was measured immediately upon removal from ice to avoid any possible release that may occur at room temperature. During the measurement, temperature increased from $1^{\circ}$ to $14^{\circ} \mathrm{C}$, but the timing was consistent resulting in reproducible $\mathrm{R}_{1}$ measurements. After dilution (1: 9 in HEPES, 1: 5 in plasma), fluorescence intensity was measured. For release in HEPES, three replicate wells of a 96-well plate were analyzed to determine Dox fluorescence at each time point, whereas 1 well was used for each time point in plasma release assay. Three separate experiments were performed at each temperature, for both HEPES and plasma release $(n=3)$ 


\section{Gd-HP-DO3A release}

Release of Gd-HP-DO3A was quantified using three methods: 1) measurement of $R_{1}$ at $0.5 \mathrm{~T}$ before size exclusion chromatography, 2) use of a calibrated relationship between $\mathrm{R}_{1}$ and contrast agent concentration after size exclusion chromatography and 3) measurements of gadolinium concentration using inductively coupled plasma-atomic emission spectroscopy (ICP-AES). Samples obtained during the simultaneous Dox and Gd-HP-DO3A release assay (see Dox release above) were used without dilution in the case of plasma release assays. For release measurements in HEPES, samples were further diluted using HEPES to a total volume of $1 \mathrm{~mL}$ and kept on ice until further analysis. $\mathrm{R}_{1}$ was measured using a custom-designed variable field $\mathrm{T}_{1}-\mathrm{T}_{2}$ analyzer (southwest Research Institute, San Antonio, TX) as previously reported [47]. To approximate $\mathrm{R}_{1}$ at $100 \%$ release, Triton ${ }^{\circledR} \mathrm{X}-100(33$ $\mu \mathrm{L}$ of $10 \% \mathrm{w} / \mathrm{w}$ in HEPES and $23 \mu \mathrm{L}$ of $25 \% \mathrm{wt} / \mathrm{wt}$ in plasma release assays) was added to one sample. This concluded quantification using method 1 for both HEPES and plasma. It is important to note that addition of Triton ${ }^{\oplus} \mathrm{X}-100$ (highest concentration tested and used was 3.5 vol\% of $25 \%$ Triton ${ }^{\circ} \mathrm{X}-100$ ) has no detectable effect on $\mathrm{R}_{1}$ in either HEPES or plasma. In HEPES, release was also quantified using methods 2 and 3. Samples were passed through Sephadex-G50 (fine) spin columns twice to remove released contents. Triton ${ }^{\oplus} \mathrm{X}-100(33 \mu \mathrm{L}$ of $10 \% \mathrm{w} / \mathrm{w}$ in HEPES) was added to each sample and $\mathrm{R}_{1}$ was once again measured using the same procedure. In order to convert the $\mathrm{R}_{1}$ reading to Gd-HP-DO3A concentration, a calibration curve was constructed relating $\mathrm{R}_{1}$ to Gd-HP-DO3A concentration. The same samples whose $R_{1}$ was measured above were then analyzed for gadolinium and phosphorus content using ICP-AES (Perkin-Elmer Plasma 40), operated at the wavelengths of 342 and $213 \mathrm{~nm}$ for gadolinium and phosphorus detection, respectively [48]. These measurements were reported as molar concentrations of Gd-HP-DO3A and as weight percent of Dox and 
gadolinium with respect to lipid, where the lipid concentration was based on the phosphorus concentration.

\section{Analysis of liposome release kinetics}

Temperature of maximum release rate (TMRR) of iLTSL was measured as the maximum of the derivative of the fluorescence-temperature curve obtained in the ThermoScan release assay. In all other assays of release, percentage release of Dox was calculated at time $t$ using the equation given below.

$$
\% \text { Release }_{t}=\frac{(a-b)}{(c-\mathbf{d})} \times 100 \% \ldots \ldots \ldots \ldots \ldots \ldots \ldots \ldots .(\text { Equation } 1)
$$

The meaning of the variables in the above equation differs in the three methods used for release measurements of Dox and the three methods of Gd-HP-DO3A release measurements. In the Dox release assays, $a$ represents the fluorescence intensity at time $t ; b$ and $d$ are equal to the fluorescence intensity at $25^{\circ} \mathrm{C}$ and $c$ is the intensity after $100 \%$ release. For the three methods used to measure release of Gd-HP-DO3A, percentage of release was calculated using the following values for the variables in Equation 1. In $\mathrm{R}_{1}$ measurements before size exclusion chromatography (method 1 ), a represents $\mathrm{R}_{1}$ at time $t ; b$ and $d$ are values of $\mathrm{R}_{1}$ at time $=0 \mathrm{~s}$ and $c$ is $\mathrm{R} 1$ after the addition of Triton ${ }^{\circledR} \mathrm{X}-100$. In measurement of Gd-HP-DO3A contained inside LTSL after size exclusion chromatography based on $\mathrm{R}_{1}$ (method 2) and ICP-AES measurements (method 3), $a$ and $c$ represent Gd-HP-DO3A at time $=0$ s, while $b$ is Gd-HP-DO3A contained in the internal compartment of the liposome at time $t$, and $d$ is Gd-HP-DO3A in a sample identical to $a$ and $c$, but one that was treated with Triton ${ }^{\oplus} \mathrm{X}-100$ before being passed through the columns (Gd-HP-DO3A removed by column). Thus, Equation 1 was modified for methods 2 and 3 to account for the fact that GdHP-DO3A released during the assay would be removed by the spin columns. 
A least-squares fit of Equation 2 to release curves for Dox and Gd-HP-DO3A (percentage release versus time) was used to calculate the release rate constant $k$. The first $10 \mathrm{~min}$ of data following initiation of release were used in the fit. In this equation, the fit constant $M a x$ indicates the maximum percentage release and $t$ represents time in min.

$$
\% \text { Release }_{t}=\operatorname{Max}\left(1-e^{-k t}\right)
$$

Release rate constant reported herein refers to the fitted value of $k$. Values of percentage release at $15 \mathrm{~s}$ and Time to $50 \%$ release were interpolated from the fit. Consequently, time to $50 \%$ release could not be measured at the lower temperatures where this level of release was not reached during the assay. Absolute average difference in percentage release was calculated as the average of differences between all of the time points in two release assays, for 10 min following initiation of release. Max was constrained to be greater than 0 , and is referred to as Release at $10 \mathrm{~min}$.

\section{Liposome stability}

Liposome stability was investigated by measuring size and Dox release kinetics (see Dox kinetic release assay above). Liposome size was measured for 7 consecutive days and Dox release in HEPES buffer was quantified on day 0 and 7 (at $25^{\circ}, 37^{\circ}$, $40^{\circ}$ and $41^{\circ} \mathrm{C}$ ). Day zero was defined as the day that the LTSLs were prepared. Liposomes were stored at $4{ }^{\circ} \mathrm{C}$ between measurements.

\section{Measurement of relaxivity}

Measurement of the longitudinal Relaxation time (T1) and calculation of the longitudinal relaxivity ( $\mathrm{r} 1)$ of heated and unheated iLTSL, as well as unencapsulated Gd-HP-DO3A was determined using a clinical $1.5 \mathrm{~T}$ MR 
scanner (Philips Medical Systems, Best, Netherlands) in 10 mM HEPES (pH 7.4, $140 \mathrm{mM} \mathrm{NaCl}, 280 \mathrm{mOsm}$ ). Gd-HP-DO3A was released from iLTSL (final concentration $=0.016-2.0 \mathrm{mM}$ Gd-HP-DO3A) by heating above the TMRR using a hot water bath $\left(55^{\circ} \mathrm{C}\right.$ for $\left.5 \mathrm{~min}\right) . \mathrm{R}_{1}$ of all of the solutions was calculated as previously reported [49] from the fit of signal intensity vs. inversion time in images obtained with an inversion recovery sequence with variable inversion time $(\mathrm{TI}=50,75,100,150,300,450,600,900,1050 \mathrm{~ms})$. Relaxivity was obtained as the slope of $\mathrm{R}_{1}$ versus Gd-HP-DO3A concentration.

\section{Phantom preparation}

Tissue mimicking agar-silica phantoms containing iLTSL were prepared using silicon dioxide powder and agarose powder ( 2 wt.\% of each). Each gel-suspended liposome phantom was cast in two stages. First, a larger phantom with a rectangular cavity was made using the higher melting point agarose/silicone gel. After this solidified, the cavity was filled with the low melting point agarose/silicone gel containing iLTSL. Gel made with higher melting temperature agarose was mixed in de-gassed $280 \pm 10 \mathrm{mOsm} 0.9 \% \mathrm{NaCl}$ solution ( $\mathrm{pH}$ adjusted to 7.4) and heated above $90^{\circ} \mathrm{C}$ for 30 min while mixing. The gel was slowly cooled at room temperature while mixing to prevent settling of silicone. Upon cooling to $45^{\circ} \mathrm{C}$, the gel was poured into a container and set to solidify on ice.

Low melting point agarose gel was used to suspend liposomes and it was prepared in a different fashion than higher melting point agarose gel. After heating above $90{ }^{\circ} \mathrm{C}$ as above, the agar-silica mixture was allowed to cool to $31{ }^{\circ} \mathrm{C}$, at which point iLTSL that was also pre- heated to $31^{\circ} \mathrm{C}$ was added and mixed with a glass rod to ensure homogeneous distribution of iLTSL in the gel. Once the iLTSL containing gel was well-mixed, it was poured into a cavity within the higher melting point agarose phantom and allowed to solidify. The procedure resulted in 
an approximate concentration of $1 \mathrm{mM} \mathrm{Gd-HP-DO3A}$ in the inner, liposomecontaining part of the phantom.

\section{MR-HIFU procedure and imaging of phantoms}

Release of iLTSL in phantoms was examined using a Sonalleve MR-HIFU treatment system (Philips Medical Systems, Vantaa, Finland). The system included a clinical 1.5 MRI scanner and an electromechanically positioned ultrasound transducer. The MR system was used to plan the therapy with 3D anatomical imaging and to guide and monitor hyperthermia with thermal imaging during treatment. Heating with MR-HIFU was achieved by focusing an ultrasound beam using a 256-element phased array focused piezoelectric ultrasound transducer immersed in a sealed tank of degassed water, running at 1.2 MHz. A single -2mm diameter HIFU focus was steered electronically (by altering the relative phases of transducer elements) [50]. Feedback control of heating relies on the proton resonance frequency shift method [51]. Temperature was raised with constant acoustic power until the mean temperature of the treatment cell increased above the cut-off temperature $\left(\mathrm{T}>42{ }^{\circ} \mathrm{C}\right)$. The treatment cell was then allowed to cool for a fixed period of time (30 s). This heat and cool cycle were then repeated to achieve the desired duration of hyperthermia. Several MR imaging sequences were used to plan the location of heating and to evaluate temperature and contrast agent release. A T2-weighted, 3D turbo spin-echo (TSE) sequence $(\mathrm{TR}=1000$ $\mathrm{ms},=80 \mathrm{~ms}, \mathrm{FA}=90^{\circ}$, TSE train length $=80,65$ slices, FOV $=280 \pm 280 \mathrm{~cm}$, $256 \pm 160$ matrix, NEX $=1$ ) was used to acquire a coronal stack. It was transferred to the HIFU workstation and used for ultrasound exposure planning. Before and after each heating, a 3D fast field echo (FFE) sequence was used to assess contrast agent release $\left(\mathrm{TR}=10 \mathrm{~ms}, \mathrm{TE}=3.8 \mathrm{~ms}, \mathrm{FA}=10^{\circ}, 44\right.$ slices, $\mathrm{FOV}=40 \pm 40 \mathrm{~cm}$, $256 \pm 256$ matrix, NEX = 10). To monitor the induced temperature elevation during each heating session, a multi-shot $\mathrm{T}_{2}$ - weighted FFE-EPI sequence was 
performed every $2.9 \mathrm{~s}\left(\mathrm{TR}=39 \mathrm{~ms}, \mathrm{TE}=19.5 \mathrm{~ms}, \mathrm{FA}=19.5^{\circ} \mathrm{C}\right.$, slice thickness $=7 \mathrm{mM}, 6$ slices, FOV $=40 \pm 40 \mathrm{~cm}, 160 \pm 160$ matrix, NEX =1). Each agarsilica gel phantom was positioned on the treatment table, and acoustic coupling was achieved with degassed water. The starting temperature before heating was approximately $13^{\circ} \mathrm{C}$.

\section{In vivo imaging of the release of contrast}

All animal experiments were performed under an approved National Institutes of Health Institutional Animal Care and Use Committee protocol. Two New Zealand white rabbits (female, $-3 \mathrm{~kg}$ ) were inoculated with $3 \times 10^{6} \mathrm{VX} 2$ cells (150 $\mu \mathrm{L}$ suspension) in the superficial thigh muscle, with ultrasound guidance. Tumor growth was monitored with ultrasound, and the experiments were performed when the tumor was greater than $1 \mathrm{~cm}$ in any dimension. Rabbits were anaesthetized with a mixture of ketamine and xylazine $(28.6 \mathrm{mg} / \mathrm{kg}$ ketamine, $4.8 \mathrm{mg} / \mathrm{kg}$ xylazine) and the marginal ear vein was catheterized. Rabbits were transferred to isoflurane anesthesia, and optical temperature probes were placed in the rectum (used to monitor animal body temperature) and the thigh muscle in the proximity of the tumor (used as input baseline temperature, prior to each sonication). The tumor-bearing limb was partly submerged in degassed de-ionized water above the HIFU transducer. A high-resolution 3D turbo spin echo scan weighted to proton density was used for treatment planning ( $\mathrm{TR}=1600 \mathrm{~ms}$, $\mathrm{TE}=30 \mathrm{~ms}$, slice thickness $=2 \mathrm{mM}, 120$ slices, FOV $=20 \pm 20 \mathrm{~cm}, 640 \pm 640$ matrix, NEX = 1). A high resolution T1-weighted 3D FFE sequence was used to assess signal intensity prior to injection, as well as before and after each sonication $\left(\mathrm{TR}=7 \mathrm{~ms}, \mathrm{TE}=3 \mathrm{~ms}, \mathrm{FA}=10^{\circ} \mathrm{C}\right.$, slice thickness $=2 \mathrm{mM}, 25$ slices, $\mathrm{FOV}=15$ $\pm 15 \mathrm{~cm}, 192 \pm 192$ matrix, NEX $=10$ ). Temperature mapping was performed with a multi-shot T2- weighted FFE-EPI sequence every $2.5 \mathrm{~s}$ (TR = $54 \mathrm{~ms}$, TE $=30 \mathrm{~ms}, \mathrm{FA}=19^{\circ} \mathrm{C}, 2$ slices, FOV $=20 \pm 20 \mathrm{~cm}, 144 \pm 144$ matrix, NEX =1). 
HIFU binary feedback control algorithm was similar to that used in phantom studies (above), with two exceptions: a region of unheated muscle was used to correct for magnetic drift, the temperature in the heated region was maintained between $40^{\circ}$ and $41^{\circ} \mathrm{C}$.

\section{Statistical analysis}

Fitted parameters were compared using the F-test. The values of percentage release were compared using Dunn's multiple comparison. Error reported for interpolated values (time to $50 \%$ release and percentage release at $15 \mathrm{~s}$ ) was calculated as the average of the upper and lower 95\% confidence intervals of the fit. Only the first $10 \mathrm{~min}$ of release curves were used for fitting. Correlation analyses of temporal particle size and polydispersity index data were performed using the Pearson correlation statistic. All fitting and statistical analyses were performed using GraphPad Prism (version 5.0 for Windows, San Diego, CA). Each curve was considered an independent sample. Results were considered significant with $p<0.05$, and two-tailed $p$-values were obtained in all cases. Pairwise comparisons with Dunn's multiple comparison test were only reported when Kruskal-Wallis showed significant differences between all tested groups to protect against type I error. Values are reported as mean \pm SEM.

\section{Results}

\section{Characterization of iLTSL}

The Z-average diameter of iLTSL liposomes in four preparations was $98 \pm 2 \mathrm{~nm}$ and polydispersity index was $0.07 \pm 0.02$. These liposomes encapsulated 4.97 \pm 0.07 wt.\% Dox based on only Dox and lipid content, or $3.52 \pm 0.05$ wt.\% Dox based on Dox, lipid and Gd- HP-DO3A. The amount of Gd-HP-DO3A encapsulated was $18.4 \pm 1.2$ wt.\% based on only Gd-HP-DO3A and lipid and 
$17.6 \pm 1.1 \mathrm{wt} . \%$ based on Dox, lipid and Gd-HP-DO3A. Using a $50 \mathrm{mg} / \mathrm{mL}$ lipid preparation and a $300 \mathrm{mM}$ Gd-HP-DO3A hydration buffer resulted in a $59 \pm 5$ $\mathrm{mM}$ Gd-HP-DO3A and $2.14 \pm 0.02 \mathrm{mg}$ Dox/mL iLTSL solution.

\section{Dox release}

Using fluorescence de-quenching, the release of Dox from iLTSL was measured in HEPES buffer as well as in human plasma. Panels A and C of Figure 2.1 show that Dox fluorescence increased gradually as temperature increased before rapid drug release occurred around TMRR of the liposomal membrane (maximum

\section{In HEPES Buffer:}
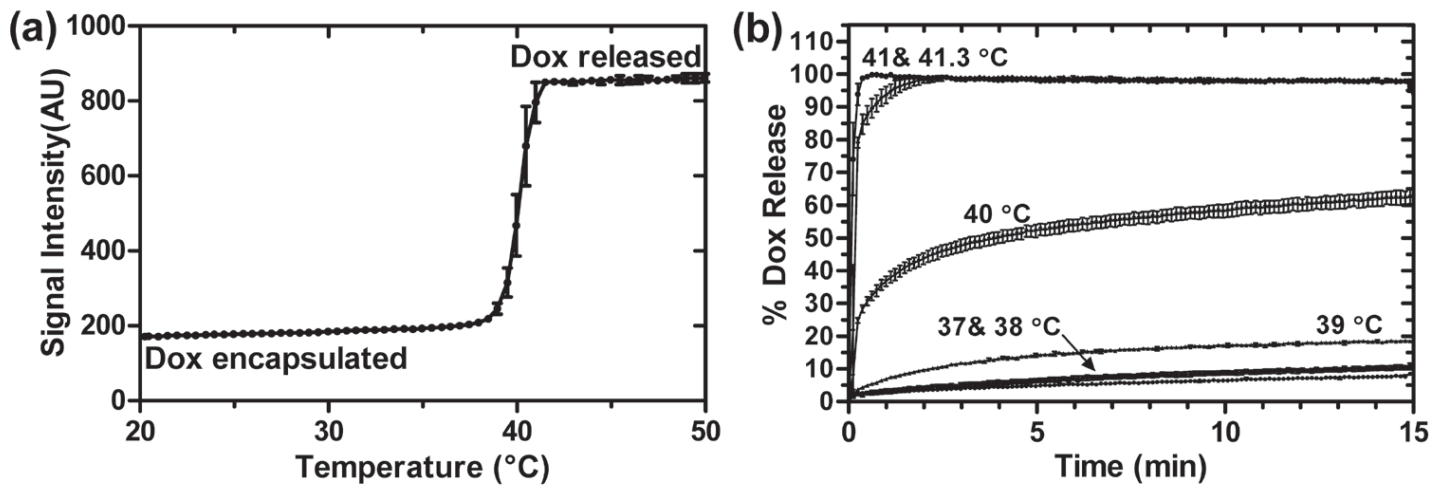

\section{In Human Plasma:}
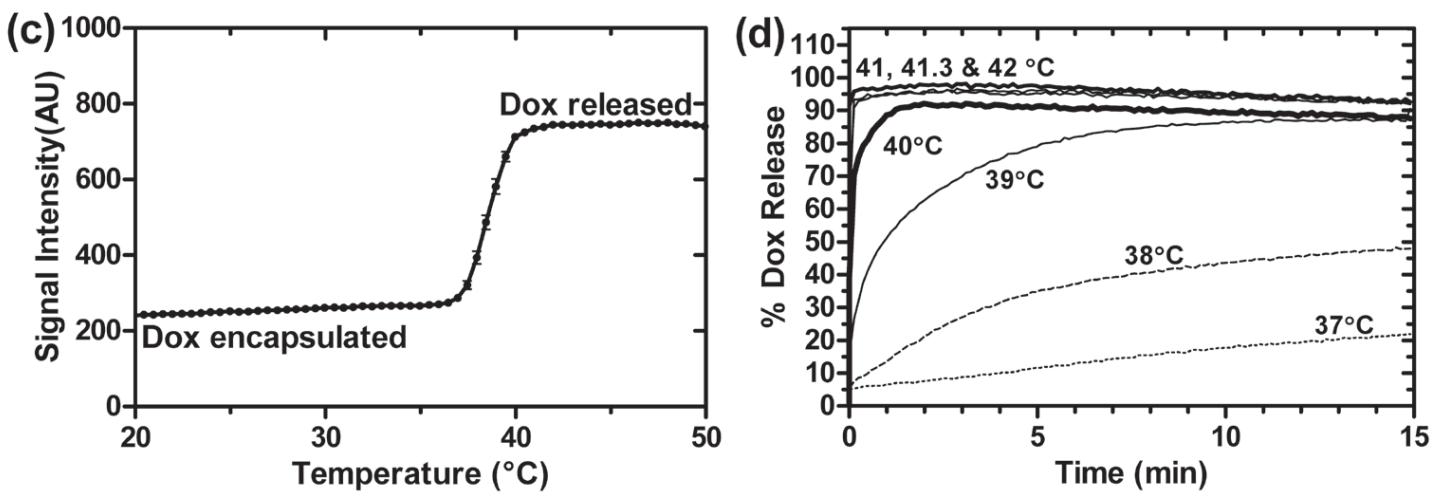

Figure 2.1 Release from iLTSL in HEPES buffer and human plasma. (a) Release of Dox as a function of temperature is shown as the sample is heated from 20 to $55^{\circ} \mathrm{C}$, at a rate of $1{ }^{\circ} \mathrm{C} /$ min. Note that in this graph, the influence of temperature and time are coupled. (b) Dox release as a function of time at a constant temperature. Percent release is calculated by assuming $100 \%$ release with Triton X-100 and $0 \%$ release at $25^{\circ} \mathrm{C}$. (c) and (d) show the same data for release in human plasma. 
ion permeability $=41.3^{\circ} \mathrm{C}$ for similar LTSL [52]). The values of TMRR in these ThermoScan assays were $40.3^{\circ} \pm 0.2^{\circ} \mathrm{C}$ in HEPES and $38.36 \pm 0.17^{\circ} \mathrm{C}$ in plasma.

When fluorescence was measured at constant temperature in Dox kinetic release assay, dox release from the LTSL was minimal $(<7-20 \%)$ at $37-39^{\circ} \mathrm{C}$ after 15 min in HEPES (Figure 2.1b). At $40{ }^{\circ} \mathrm{C}$ there was $-30 \%$ released in less than 20 $s$ followed by a more gradual release. Near the TMRR, complete release $(>90 \%)$ occurred in approximately $7 \mathrm{~s}$ (by the first measured time point) at $41{ }^{\circ} \mathrm{C}$ and 41.3 ${ }^{\circ} \mathrm{C}$. Rapid and complete release of Dox also occurred in plasma at these higher temperatures (Figure 2.1d). The release rate constants in plasma at $39^{\circ}-41{ }^{\circ} \mathrm{C}$ were greater than those in buffer

\section{Gd-HP-DO3A release}

Release of Gd-HP-DO3A was measured with ICP-AES and compared to Dox release in the same sample, as shown in Figure 2.2a. The difference in percentage release between amounts of Dox and Gd-HP-DO3A was less than 20\% for all time points, with the exception of 2 time points at $40{ }^{\circ} \mathrm{C}$ (at 2 and 3 min, Figure 2.2a). Differences in the magnitude of Dox and Gd-HP-DO3A percentage release were not significant ( $p>0.05$, Dunn's multiple comparison) over $10 \mathrm{~min}$ following initiation of release (mean absolute differences were $0.9 \pm 0.2 \%, 5 \pm 1 \%$ and 0.5 $\pm 1 \%$ for $37^{\circ}, 40^{\circ}$ and $41.3{ }^{\circ} \mathrm{C}$, respectively). This lack of difference in release was especially evident in the first minute of release (Figure 2.2b). Release of Gd-HP-DO3A was also determined with $\mathrm{R}_{1}$ measurements (at $0.5 \mathrm{~T}$ ) before size-exclusion chromatography.

Agreement between these $\mathrm{R}_{1}$-based measurements and ICP-AES measurements is shown in Supplemental Figure 2. The temperature measured during simultaneous Dox and Gd-HP-DO3A release assay is shown in Supplemental Figure 3, 

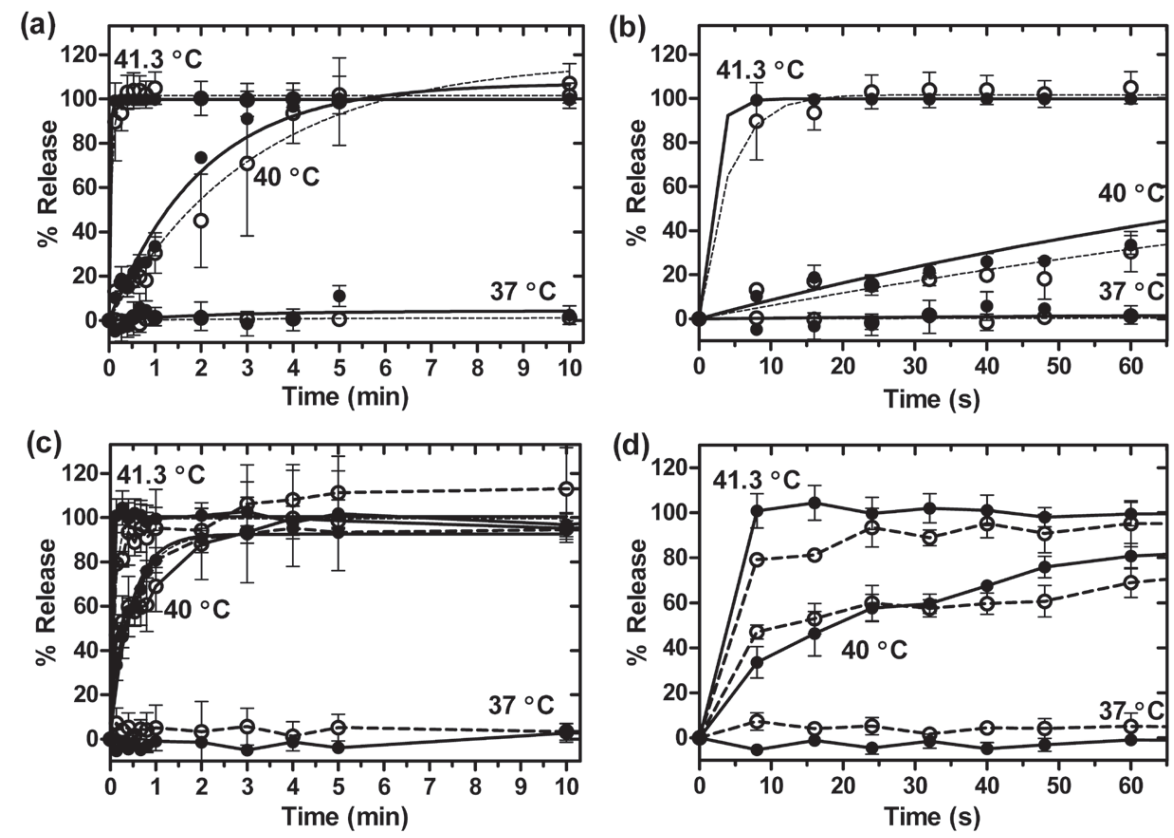

Figure 2.2 Release of Dox and Gd-HP-DO3A from iLTSL. Release in HEPES: (a) Percent release vs. time and fitted curves for Dox $\left(\mathrm{o}_{--}\right)$and Gd-HP-DO3A $(\bullet-)$ over $10 \mathrm{~min}$. (b) Release in the first minute. Percent release values were not significantly different between Dox and Gd-HP-DO3A over 10 minutes ( $p>0.05$, Dunn's multiple comparison). Release in plasma: (c) Percent release vs time for Dox and Gd-HP-DO3A over $10 \mathrm{~min}$. (d) Release in the first minute. Percent release values were not significantly different between Dox and Gd-HP-DO3A in either HEPES or plasma ( $p>0.05$, Dunn's multiple comparison). Each point represents the mean of 3 experiments \pm SEM.

demonstrating a drop-in temperature of $0.5-1{ }^{\circ} \mathrm{C}$ that recovers approximately 3 min after addition of liposomes. Ratio of gadolinium to phosphate indicated that relative to the lipid concentration, concentration of contrast agent after size exclusion chromatography decreased faster with increasing temperature.

In plasma, a comparison of Gd-HP-DO3A and Dox release in the same sample was also performed, with similar results (Figure 2.2c and d). Just as with HEPES, all but two points showed less than 20\% difference in percentage release of Dox and Gd-HP-DO3A, and overall, differences in release were not significant over the first 10 min of release ( $p>0.05$, Dunn's multiple comparison). The first minute of release also showed close agreement in the kinetics of Dox and Gd-HP-DO3A release. 


\section{Stability of iLTSL}

Liposome stability, indicated by lack of temporal dependence of either particle size or polydispersity index ( $p>0.05$, Pearson), was relatively constant for one week (95 $\mathrm{nm}$ size, polydispersity index $=0.05$ at day 0 and $102 \mathrm{~nm}$ size, polydispersity index $=0.11$ at day $7, n=3)$. Furthermore, the Dox release rate remained unchanged a week after synthesis and storage of iLTSL at $4{ }^{\circ} \mathrm{C}$ (Figure 2.3a). Overall, there was slightly less release on day 7 (mean decrease in release of $0.11 \pm 0.14$ to 1.83 $\pm 0.05 \%$ over $10 \mathrm{~min}$ heating), as indicated by the difference in percentage release (Figure 2.3b). This difference was significant at $37^{\circ} \mathrm{C}(p<0.05$, Dunn's multiple comparison test), but not at other temperatures.

\section{Imaging and spatial control of release}

Image-ability-The ability to image content release from iLTSL was first examined through longitudinal relaxivity measurements. Imaging of a series of dilutions of iLTSL at $1.5 \mathrm{~T}$ showed that relaxivity of preheated iLTSL was $4.01 \pm 0.01$ $\mathrm{mM}^{-1} \mathrm{~s}^{-1}$, which was significantly higher $(p<0.0001, \mathrm{~F}$-test $)$ than the relaxivity of unheated solutions $(1.95 \pm 0.05 \mathrm{mM}-1 \mathrm{~s}-1$, Figure 2.4).
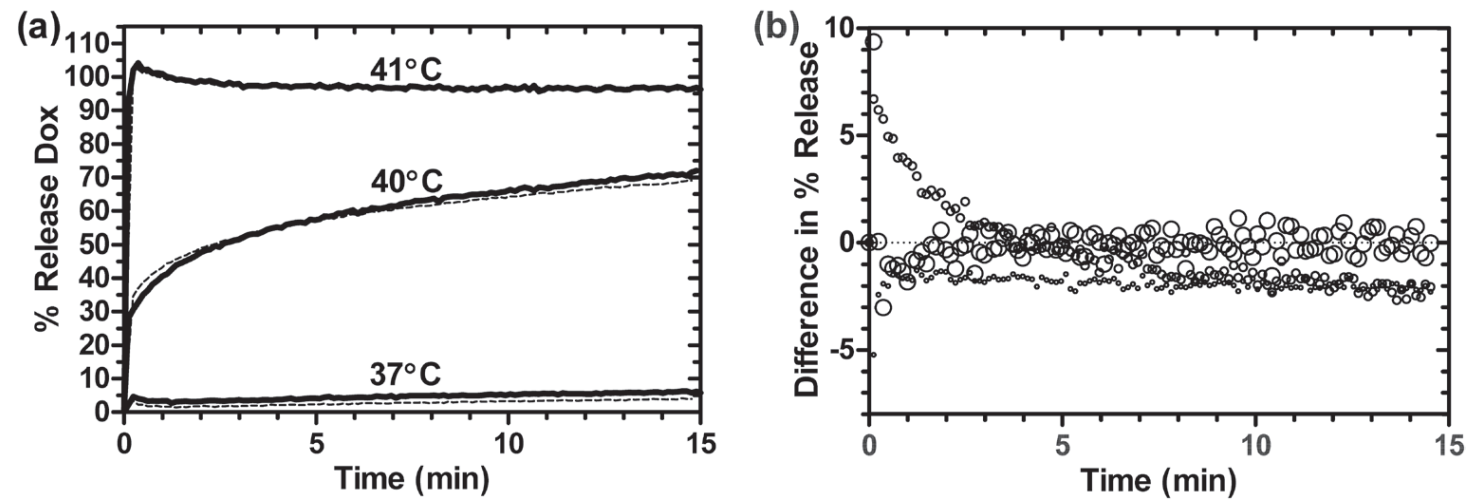

Figure 2.3 Stability of Gd-LTSL-Dox. (a) Release of doxorubicin immediately (dashed line) and 7 days after synthesis (solid line) of Gd-LTSL-Dox at 37, 40 and $41{ }^{\circ} \mathrm{C}$ in HEPES buffer. (b) Point-by-point difference between release curves at 37, 40 and $41{ }^{\circ} \mathrm{C}$ obtained 1 week apart (\% release at day $0-\%$ release at day 7$)$. Symbol size indicates temperature: $37{ }^{\circ} \mathrm{C}$ is smallest $\left({ }_{0}^{--}\right)$and $41^{\circ} \mathrm{C}$ is largest $(\bullet-)$. 


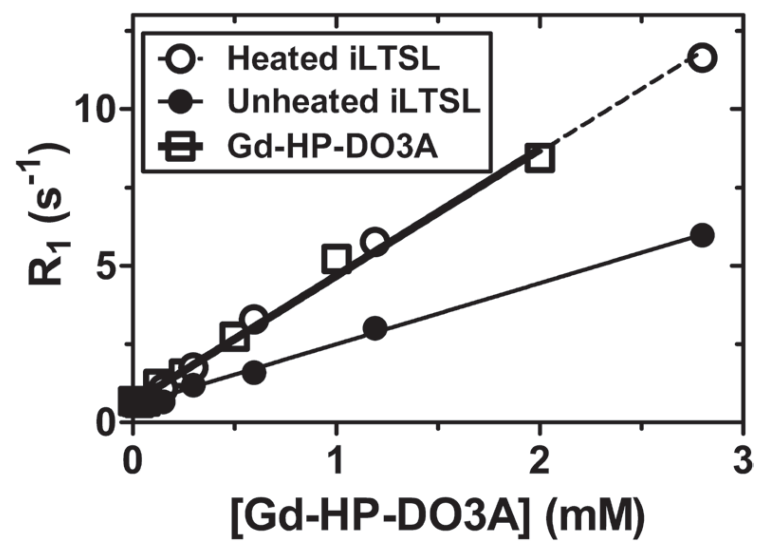

Figure 2.4 Relaxation rate (R1) versus concentration of Gd-HP-DO3A at 1.5T. Gd-LTSL-Dox solutions were heated in a water bath $\left(55^{\circ} \mathrm{C}\right.$ for $5 \mathrm{~min}$.) to release Gd-HP-DO3A and Dox. The resulting relaxivity (slope) values for heated and unheated iLTSL were $4.01 \pm 0.01$ and $1.95 \pm$ $0.05 \mathrm{mM}^{-1} \mathrm{~s}^{-1}$, respectively, and were significantly different ( $\mathrm{p}<0.001, \mathrm{~F}$-test). Relaxivity of Gd-HPDO3A $\left(4.05 \pm 0.14 \mathrm{mM}^{-1} \mathrm{~s}^{-1}\right)$ was not significantly different from that of heated iLTSL $(\mathrm{p}=0.85$, F-test). R2 > 0.992 for all fitted data.

\section{Spatial control of liposomal release}

Signal intensity increased approximately $36.4 \pm 0.3 \%$ in regions of a gel-suspended liposome phantom heated with MR-HIFU above TMRR, compared to the surrounding gel that contained iLTSL but was not heated (Figure 2.5). The three shapes in Figure 2.5 reflect pre-programmed trajectories where the HIFU energy was focused demonstrating the ability to yield conformal content release.

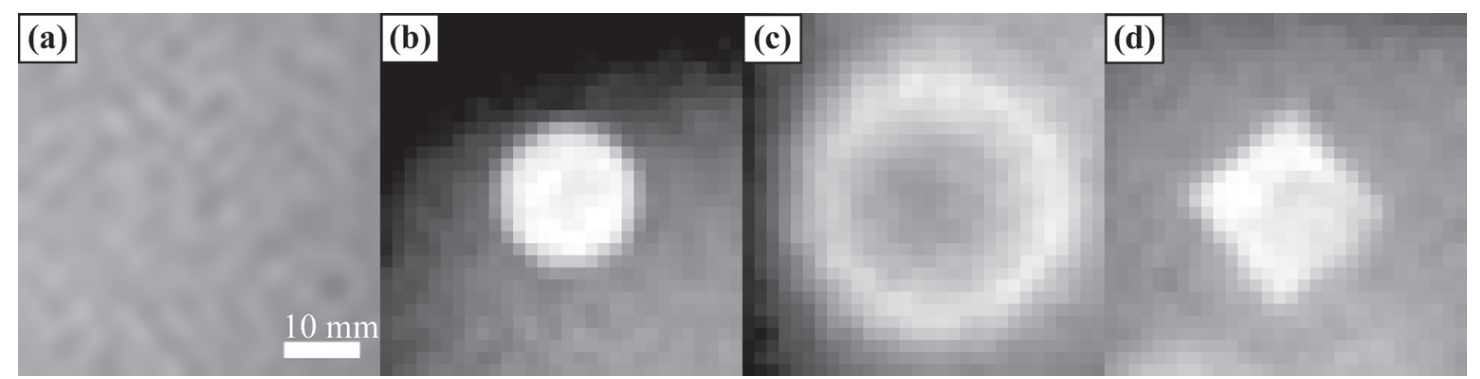

Figure 2.5 Location of release of iLTSL contents can be controlled and visualized with MR-HIFU. iLTSL suspended in 2\% agar-silica demonstrated baseline intensity (a) until heated above the Tm of the liposome with MR-HIFU (b-d). The bright shapes result from different sizes and shapes of regions that were heated with MR-HIFU. 


\section{Simultaneous imaging of content release and temperature}

In a separate experiment, real-time monitoring of temperature and content release from iLTSL was investigated by heating a gel-suspended liposome phantom with MR-HIFU (Figure 2.6). Phase images were used for MR thermometry and magnitude images were used to measure MR signal intensity. During and after heating, MR-signal intensity increased $36 \pm 4 \%$ to approximately the same magnitude as the preheated region. The signal intensity of a volume that was unheated remained at its initial level. The temperature fluctuation with HIFU application can be appreciated in Figure 2.6b, increasing during the heating and decreasing when no power was applied. The duration of each cooling cycle was $30 \mathrm{~s}$, which corresponds to the periods of cooling detected with MR thermometry in Figure 2.6b.

\section{In vivo feasibility of iLTSL delivery with MR-HIFU}

The ability of iLTSL to provide sufficient contrast using MR-HIFU was tested in a rabbit thigh tumor model. Upon injection, signal increased $20-40 \%$ in the
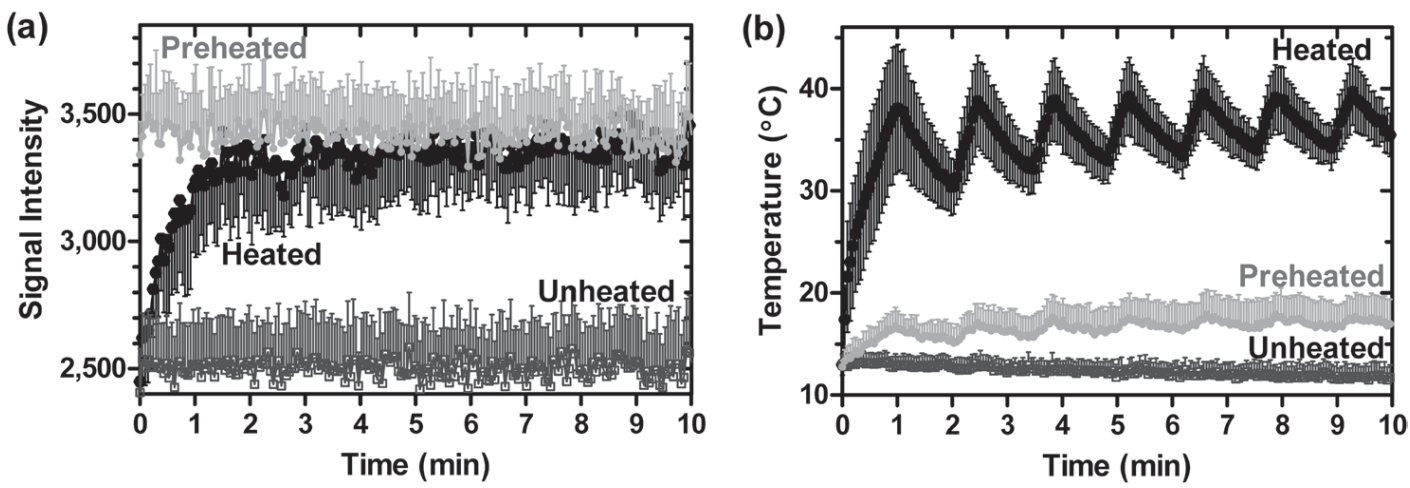

Figure 2.6 MR signal intensity during heating with MR-HIFU of a silica-agarose gel phantom containing iLTSL. (a) MR signal intensity in heated, unheated, as well as preheated regions. (b) Temperature in the same regions, as measured with MR thermometry. Signal intensity of the region where the MR-HIFU energy is focused increased most likely due to Gd-HP-DO3A release. The signal intensity in a region that has been previously heated did not change drastically but remained high relative to unheated regions. An unheated region demonstrated stable and relatively low signal intensity. Regions of interest contained $20-45$ voxels. Mean signal temperature \pm SEM is shown. 
tumor and remained stable for the 10 min prior to heating. Following each of the four 10 min heating sessions the signal in the heated portion of the tumor increased. After the fourth heating, the signal intensity in the tumor increased 9-22\% when compared to immediately after injection. The heated portion of the tumor demonstrated the greatest signal increase (30-60\%). Adjacent unheated muscle signal intensity increased $20-25 \%$ following injection but remained stable following all of the heating sessions. This study was repeated twice with similar trends.

\section{Discussion}

The image-able liposome reported herein incorporated both drug and contrast agent in the interior compartment of liposome, enabling real-time monitoring of released drug with MRI in a heated site. Prior to developing this iLTSL formulation, we identified a set of key design criteria for this liposome, including stability (size and release profiles), image-ability (ability to report on content release in MRI), and ease of clinical translation.

\section{Stability}

iLTSL stability was assured by optimizing the liposome hydrating solution that contains contrast agent and Dox loading buffer. The identity and concentration of both constituents must be properly balanced. Native osmolarity of ProHance ${ }^{\circledast}$ (Gd-HP-DO3A) was 621 mOsm while another common contrast agent, Magnevist $^{\mathrm{TM}}$, had an osmolarity of $1960 \mathrm{mOsm}$ for the same $500 \mathrm{mM}$ contrast agent concentration. The lower osmolarity for Gd-HP- DO3A allowed for more contrast agent to be loaded while maintaining stability of the liposome. A 300-mM citrate buffer $(-570 \mathrm{mOsm})$ is commonly used concentration range to actively load Dox into liposomes [53]. In the formulation reported herein, the citrate 
buffer was reduced to $100 \mathrm{mM}$ to limit osmolarity inside the liposome when combined with $300 \mathrm{mM}$ Gd-HP-DO3A (final osmolarity = -500 mOsm), while at the same time retaining the ability to load $5 \mathrm{wt} . \%$ Dox. The true osmolarity of the solution inside the liposomes following Dox loading is not known, since Dox forms fibrous structures inside the liposomes due to electrostatic interactions between Dox and citrate ions [54]. The osmolarity limit of 500-550 mOsm was empirically determined: loading solutions of greater osmolarity resulted in larger liposomes $(>115 \mathrm{~nm})$ that suggested potential instability (data not shown). The choice of $300 \mathrm{mM}$ Gd-HP-DO3A and $100 \mathrm{mM}$ citrate buffer yielded a stable particle size distribution and drug release over one week in storage (Figure 2.3). Not only the hydration buffer osmolarity, but lipid components may influence stability. A different formulation recently showed similar release properties and improved stability at body temperature [55].

\section{Image-ability}

iLTSL image-ability was optimized by selecting a proper ratio of Gd-HPDO3A to Dox to provide an MR signal increase while dosing Dox in a clinically relevant concentration. The balance between image-ability (Gd-HP-DO3A) and therapeutic efficacy (Dox), while maintaining stability, is the major consideration for optimization of a drug plus contrast agent formulation. Although greater amounts of drug have been loaded into image-able liposomes [56], the selection of a relatively low drug: lipid ratio of 5 wt.\% ensured a [Gd-HP- DO3A] high enough for imaging in vivo. This procedure yielded liposomes that would deliver $0.15 \mathrm{mmol} / \mathrm{kg}$ of Gd-HP-DO3A at the commonly used $5 \mathrm{mg} / \mathrm{kg}$ intravenous dose of Dox-loaded LTSL in mice (approximately $25 \mathrm{mg} / \mathrm{m}^{2}, 2$ times lower than human MTD of $50 \mathrm{mg} / \mathrm{m}^{2}$ ) [18], which is sufficient to increase MR signal significantly. Previously reported increase of water exchange across the liposome membrane with temperature increased pre-release $\mathrm{R}_{1}$, thus attenuating detectability of release 
with TSLs [44]. iLTSLs demonstrate relaxivity $>1 \mathrm{mM}^{-1} \mathrm{~s}^{-1}$ at room temperature, suggesting that the water exchange rate does impact relaxivity when no release occurred. However, relaxivity at body temperature was not measured and the contents of liposomes investigated herein differ from those previously reported [44]. Therefore, it is unclear to what extent the water exchange rate with iLTSL will decrease the difference in relaxivity before and after release in a physiological setting. Thus the 2 -fold increase in relaxivity after contrast agent release from the liposome (Figure 2.4) revealed the utility of this formulation in reporting on liposomal content release.

\section{Clinical translation}

Clinical translation of iLTSL may be eased by selecting a liposomal formulation with constituents that have a history of clinical use and safety. The MR contrast agent, Gd-HP-DO3A, is currently FDA-approved for imaging abnormal vascularity in the central nervous system and head and neck. Furthermore, Gd-HP-DO3A has demonstrated the lowest incidence of nephrogenic systemic toxicity (NSF) for Gd-based contrast agents $[57,58]$. Lastly, the selected lipid components are similar in identity but not in ratio to a commercial formulation, ThermoDox ${ }^{\oplus}$, (Celsion, Columbia, MD) that has been tested in various clinical trials $[18,59,60]$.

\section{Kinetics of release of Dox and Gd-HP-DO3A}

While it is preferable to directly image the desired drug for image-guided drug delivery, this is often not possible without chemically modifying the drug. We hope to use co-encapsulated Gd-HP-DO3A as a surrogate of Dox release from a liposome. The release of Dox and Gd-HP-DO3A from the same liposome was evaluated in HEPES buffer and in human plasma without a profound difference for most time points at 37,40 and $41^{\circ} \mathrm{C}$ (Figure 2.2). Increased relaxivity with 
heating was also demonstrated (Figure 2.4), which resulted in greater signal intensity (Figure 2.5). Taken together, these data suggest that an increase in signal intensity from contrast agent release can be used as a surrogate for drug release from iLTSL.

Release of Dox in plasma was faster than in HEPES buffer at temperatures below TMRR. Tables I and II shows a quantitative assessment of the influence of the presence of plasma on release of both Dox and Gd-HP-DO3A. For example, the release rate constant, time to $50 \%$ release and release at $15 \mathrm{~s}$ provide ways of comparing the rates of drug and contrast release. All of these data point to Dox being released faster in plasma in the intermediate range of temperatures $\left(39-41^{\circ} \mathrm{C}\right)$. Furthermore, a comparison of values of TMRR in Table 1 shows that maximum rate of release of Dox occurs at a lower temperature in plasma. Plasma has been shown to affect liposomal release kinetics [61], and recently it was reported that lysolecithin is lost from LTSL in plasma, and that this phenomenon may play an important role for in vivo release kinetics of such liposomes [62]. These observations warrant further investigation into effects of plasma components on TSL release. They also highlight the need to perform release assays of TSLs in plasma to estimate in vivo performance. Release of Gd-HP-DO3A from liposomes was quantified with several methods, and agreement of results from these methods shows that the simplest of the methods could be used in future studies. The main body of this work used ICP-AES, which is a direct measurement of gadolinium. However, this method required removal of released Gd-HP-DO3A from solution using size exclusion chromatography in two steps. Our data suggest that simple $\mathrm{R}_{1}$ measurements of aliquots collected during the release assay provide an equivalent estimate of percentage release (Supplemental Figure 2). Thus, using this simplified method, the kinetics of Dox and Gd-HP-DO3A were measured in plasma, where separation of released and encapsulated contrast agent would be difficult. This 
assay (Figure 2.2) demonstrates that both the contrast agent and Dox are not appreciably released in either HEPES buffer or plasma at $37^{\circ} \mathrm{C}$, with the rate and extent of release rising with temperature (Figure 2.2 and Table II). A number of methods were used to quantify and describe release of liposomal contents, such as the release rate constant, time to $50 \%$ release and release at $15 \mathrm{~s}$. For practical considerations, the most important factor is the amount of drug and/or contrast agent released during transit through the treatment volume, as suggested by recent modelling work [63]. Mean transit time in tumors is typically less than $10 \mathrm{~s}$ [64], suggesting that drug release must occur in this time window for optimal tumor accumulation. Figure 2.2 demonstrated no appreciable difference in release of drug and contrast agent on this short time scale, supporting the concept of imaging contrast agent release as a surrogate of drug release. In fact, a 7-s transit time is sufficient for near-maximal content release at $41^{\circ} \mathrm{C}$ in plasma (Figure 2.1d). While fast release is important in the heated region, the LTSLs must also stably encapsulate their contents at body temperature. Limited release at $37^{\circ} \mathrm{C}(<15 \%$ in 15 min in plasma) ensured that the majority of drug arrives to the heated region stably encapsulated inside iLTSL during a typical hyperthermia treatment (Figure 2.1d). Further improvement in plasma stability may increase delivery of drug to a heated region.

\section{Drug dose painting using MR-HIFU and iLTSL}

The ability of iLTSL to provide sufficient contrast with MR-HIFU was evaluated in a preliminary study that showed MR signal intensity increase after iLTSL injection, followed by further increases after each 10-min hyperthermia treatment (Figure 2.7). Since Dox and Gd-HP-DO3A release are well correlated, it is our hope that Dox concentration may be related to MR signal changes. However, since GdHP-DO3A and Dox have different physicochemical properties, a computational model that takes this into account may be required [63]. Correlation of MR 


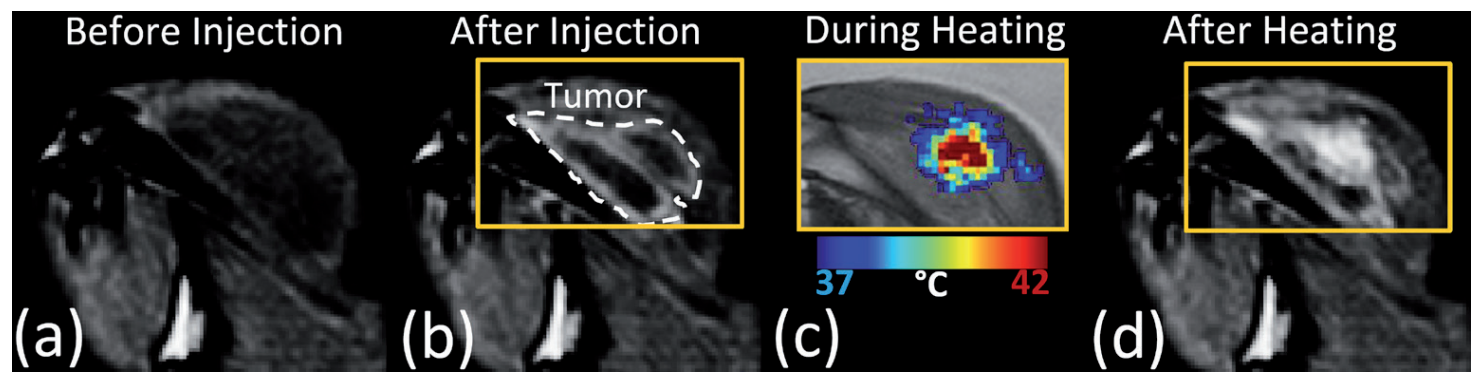

Figure 2.7 MR signal intensity before and after iLTSL injection and heating with MR-HIFU. Signal intensity (A) before iLTSL injection and (B) after iLTSL injection. (C) Example of temperature map during heating, overlaid on signal intensity obtained with a treatment planning proton density weighted scan. (D) Signal intensity after four 10-minute heating sessions. Note that (A), (B) and (D) depict T1-wighted images whereas (C) shows a proton density weighted image.

signal intensity and Dox concentration will be the subject of future investigations. Two in vivo studies recently addressed the possibility of drug dose painting with LTSLs co-loaded with an MR contrast agent and Dox [38, 39]. However, the hyperthermia system used in these studies could not quickly adjust temperature distribution in the heated volume. Real-time adjustment of treatment would best be accomplished through dynamic imaging during heating as demonstrated in phantoms (Figure 2.6). For in vivo applications, we believe quantifying drug release on a voxel-by-voxel basis may provide useful information to optimize a treatment.

Recent development and therapeutic clinical use of MR-HIFU may provide a method for using image-able temperature-sensitive liposomes for drug dose painting with higher precision and accuracy than previous hyperthermia applicators (4-mm regions have been reliably heated with temperature feedback [32]). The MR-HIFU system we adopted for mild hyperthermia is currently in clinical trials for ablative treatment of uterine fibroids with MR thermometry feedback and other systems have already been approved by FDA [25]. The MRHIFU system's ability to dose paint with various shapes, control temperature and monitor liposome release is demonstrated in Figures 2.5 and 2.6. The system is 
able to heat a tumor in vivo and cause preferential signal intensity increases in the heated region, presumably due to contrast agent release from iLTSL (Figure 2.7). However, the MRI sequence used for simultaneous temperature and contrast agent release monitoring (Figure 2.6) was optimized only for temperature imaging, which resulted in $\mathrm{T}^{*}$-weighted images. Thus, there is a clear need for an imaging approach with greater $\mathrm{T}_{1}$ contrast along with satisfactory temperature feedback for MR-HIFU. Combination of a precise hyperthermia applicator, MR thermometry, $\mathrm{T}_{1}$-weighted imaging, computational modelling, and image-able LTSLs with suitable properties may realize a dose painting paradigm for treatment of cancer and other diseases. Such spatially tuned targeting of cancer and other locally dominant diseases may provide a novel dimension for drug delivery that can take advantage of a device specially optimized for drug delivery. Furthermore, knowledge of the location of drug release may address clinical deficiencies by facilitating future treatments.

\section{Conclusion}

This work demonstrated preparation and characterization of a novel MR imageable liposome formulation co-loaded with Dox and an MR contrast agent, GdHP-DO3A. The design of the formulation ensured stability during storage and image-ability of content release. Monitoring and spatial control of liposomal content release was demonstrated with MR-HIFU in tissue-mimicking phantoms in real time, as well as after heating in vivo. 


\section{References}

1. Allen, T.M. and P.R. Cullis, Drug delivery systems: entering the mainstream. Science, 2004. 303(5665): p. 1818-22.

2. Tilcock, C., Delivery of contrast agents for magnetic resonance imaging, computed tomography, nuclear medicine and ultrasound. Adv. Drug Deliv. Rev., 1999. 37(1-3): p. 33-51.

3. Maeda, H., L.W. Seymour, and Y. Miyamoto, Conjugates of anticancer agents and polymersadvantages of macromolecular therapeutics in vivo. Bioconjugate Chem., 1992. 3(5): p. 351362.

4. Matsumura, Y. and H. Maeda, A new concept for macromolecular therapeutics in cancer chemotherapy-mechanism of tumoritropic accumulation of proteins and the antitumor agent SMANCS. Cancer Res., 1986. 46(12): p. 6387-6392.

5. Northfelt, D.W., et al., Doxorubicin encapsulated in liposomes containing surface-found polyethylene glycol: pharmacokinetics, tumor localization, and safety in patients with AIDSrelated Kaposi's sarcoma. J. Clin. Pharmacol., 1996. 36(1): p. 55-63.

6. Allen, T.M., Ligand-targeted therapeutics in anticancer therapy. Nat. Rev. Cancer, 2002. 2(10): p. 750-63.

7. Negussie, A.H., et al., Synthesis and in vitro evaluation of cyclic NGR peptide targeted thermally sensitive liposomes. J. Control. Release, 2010. 143(2): p. 265-73.

8. Simoes, S., et al., On the formulation of $p H$-sensitive long circulation times. Adv. Drug Deliv. Rev., 2004. 56(7): p. 947-965.

9. Lokling, K.E., et al., Biodistribution of $p H$-responsive liposomes for MRI and a novel approach to improve the pH-responsiveness. J. Control. Release, 2004. 98(1): p. 87-95.

10. Shum, P., J.M. Kim, and D.H. Thompson, Photo-triggering of liposomal drug delivery systems. Adv. Drug Deliv. Rev., 2001. 53(3): p. 273-284.

11. Spratt, T., B. Bondurant, and D.F. O’Brien, Rapid release of liposomal contents upon photoinitiated destabilization with UV exposure. Biochim. Biophys. Acta-Biomembr., 2003. 1611(1-2): p. 35-43.

12. Jensen, S.S., et al., Secretory phospholipase A(2) as a tumor-specific trigger for targeted delivery of a novel class of liposomal prodrug anticancer ether-lipids. Mol. Cancer Ther., 2004. 3(11): p. 1451-1458.

13. Pak, C.C., et al., Elastase activated liposomal delivery to nucleated cells. Biochim. Biophys. Acta-Biomembr., 1999. 1419(2): p. 111-126.

14. Yatvin, M.B., et al., Design of Liposomes for enhanced local release of drugs by hyperthermia. Science, 1978. 202(4374): p. 1290-1293.

15. Needham, D., et al., A new temperature-sensitive liposome for use with mild hyperthermia: characterization and testing in a human tumor xenograft model. Cancer Res., 2000. 60(5): p. 1197-1201.

16. Lindner, L.H., et al., Novel temperature-sensitive liposomes with prolonged circulation time. Clin. Cancer Res., 2004. 10(6): p. 2168-2178.

17. Hauck, M.L., et al., Phase I trial of doxorubicin-containing low temperature sensitive liposomes in spontaneous canine tumors. Clin. Cancer Res., 2006. 12(13): p. 4004-4010.

18. Poon, R.T.P. and N. Borys, Lyso-thermosensitive liposomal doxorubicin: a novel approach to enhance efficacy of thermal ablation of liver cancer. Expert Opi. Pharmaco., 2009. 10(2): p. 333-343. 
19. Staruch, R., R. Chopra, and K. Hynynen, Investigations into thermally mediated drug delivery using a preclinical system for MRI-guided focused ultrasound. AIP Conf. Proc., 2011. 1359(10th International Symposium on Therapeutic Ultrasound, 2010): p. 163-167.

20. Harris, E., Industry Update: The latest developments in therapeutic delivery. Ther. Deliv., 2013. 4(10): p. 1229-1234.

21. May, J.P. and S.-D. Li, Thermosensitive liposomes in cancer therapy. Recent Pat. Biomed. Eng., 2012. 5(2): p. 148-158.

22. Swenson, C.E., et al., Increased duration of heating boosts local drug deposition during radiofrequency ablation in combination with thermally sensitive liposomes (ThermoDox) in a porcine model. PLoS One, 2015. 10(10): p. e0139752/1-e0139752/15.

23. Ter Haar, G. and C. Coussios, High intensity focused ultrasound: physical principles and devices. Int. J. Hyperther., 2007. 23(2): p. 89-104.

24. McDannold, N., et al., MRI evaluation of thermal ablation of tumors with focused ultrasound. J. Magn. Reson. Imaging, 1998. 8(1): p. 91-100.

25. Stewart, E.A., et al., Clinical outcomes of focused ultrasound surgery for the treatment of uterine fibroids. Fertil. Steril., 2006. 85(1): p. 22-29.

26. Kaye, E.A., et al., Feasibility study on MR-guided high-intensity focused ultrasound ablation of sciatic nerve in a swine model: preliminary results. Cardiovasc. Interv. Radiol., 2015. 38(4): p. 985-992.

27. Ellis, S., et al., Clinical applications for magnetic resonance guided high intensity focused ultrasound (MRgHIFU): present and future. J. Med. Imaging. Radiat. Oncol., 2013. 57(4): p. 391-9.

28. Kim, Y.S., H.K. Lim, and H. Rhim, Magnetic resonance imaging-guided high-intensity focused ultrasound ablation of uterine fibroids: effect of bowel interposition on procedure feasibility and a unique bowel displacement technique. Plos One, 2016. 11(5): e 0155670.

29. Venkatesan, A.M., et al., Magnetic resonance imaging-guided volumetric ablation of symptomatic leiomyomata: correlation of imaging with histology. J. Vasc. Interv. Radiol., 2012. 23(6): p. 786-794.

30. Zhang, L., et al., Feasibility of magnetic resonance imaging-guided high intensity focused ultrasound therapy for ablating uterine fibroids in patients with bowel lies anterior to uterus. Eur. J. Radiol., 2010. 73(2): p. 396-403.

31. Tempany, C.M.C., et al., MR imaging-guided focused ultrasound surgery of uterine leiomyomas: a feasibility study. Radiol., 2003. 226(3): p. 897-905.

32. Enholm, J.K., et al., Improved volumetric MR-HIFU ablation by robust binary feedback control. IEEE. Trans. Biomed. Eng., 2010. 57(1): p. 103-113.

33. Schramm, W., D. Yang, and D. Haemmerich, Contribution of direct heating, thermal conduction and perfusion during radiofrequency and microwave ablation. Conf. Proc. IEEE. Eng. Med. Biol. Soc., 2006. 1: p. 5013-6.

34. Freccero, C., et al., Laser Doppler perfusion monitoring of skin blood flow at different depths in finger and arm upon local heating. Microvasc. Res., 2003. 66(3): p. 183-9.

35. Tilcock, C., et al., Liposomal Gd-DTPA-preparation and characterization of relaxivity. Radiol., 1989. 171(1): p. 77-80.

36. Ghaghada, K., et al., T1 relaxivity of core-encapsulated gadolinium liposomal contrast agentseffect of liposome size and internal gadolinium concentration. Acad. Radiol., 2008. 15(10): p. 1259-1263.

37. Mulder, W.J.M., et al., A liposomal system for contrast-enhanced magnetic resonance imaging of molecular targets. Bioconjugate Chem., 2004. 15(4): p. 799-806. 
38. Laurent, S., et al., Paramagnetic liposomes: inner versus outer membrane relaxivity of DPPC liposomes incorporating lipophilic gadolinium complexes. Langmuir, 2008. 24(8): p. 43474351 .

39. KB, G., et al. New dual mode gadolinium nanoparticle contrast agent for magnetic resonance imaging. Plos One, 2009. 4(10): e7628.

40. Koenig, S.H., et al., Permeability of liposomal membranes to water-results from the magneticfield dependence of $T 1$ of solvent protons in suspensions of vesicles with entrapped paramagnetic ions. Magn. Reson. Med., 1992. 23(2): p. 275-286.

41. Frich, L., et al., Experimental application of thermosensitive paramagnetic liposomes for monitoring magnetic resonance imaging guided thermal ablation. Magn. Reson. Med., 2004. 52(6): p. 1302-1309.

42. Viglianti, B.L., et al., In vivo monitoring of real time tissue pharmacokinetics of liposomeldrug concentration using MRI. Radiol., 2002. 225: p. 647-648.

43. Ponce, A.M., et al., Magnetic resonance imaging of temperature-sensitive liposome release: drug dose painting and antitumor effects. J. Natl. Cancer Inst., 2007. 99(1): p. 53-63.

44. de Smet, M., et al., Temperature-sensitive liposomes for doxorubicin delivery under MRI guidance. J. Control. Release, 2010. 143(1): p. 120-127.

45. Ponce, A., et al., Magnetic resonance imaging of temperature-sensitive liposome release: drug dose painting and antitumor effects. J. Natl. Cancer Inst., 2007. 99(1): p. 53-63.

46. Mayer, L.D., M.B. Bally, and P.R. Cullis, Uptake of adriamycin into large unilamellar vesicles in response to a pH gradient. Biochim. Biophys. Acta, 1986. 857(1): p. 123-6.

47. Bryant, L.H., et al., Synthesis and relaxometry of high-generation $(G=5,7,9$, and 10$)$ PAMAM dendrimer-DOTA-gadolinium chelates. J. Magn. Reson.Imaging, 1999. 9(2): p. 348-352.

48. Molinelli, A.R., et al., Effect of metal removal on the toxicity of airborne particulate matter from the Utah Valley. Inhal. Toxicol., 2002. 14(10): p. 1069-86.

49. Bluml, S., et al., Spin-lattice relaxation-time measurement by means of a turboflash technique. Magn. Reson. Med., 1993. 30(3): p. 289-295.

50. Salomir, R., et al., Local hyperthermia with MR-guided focused ultrasound: spiral trajectory of the focal point optimized for temperature uniformity in the target region. J. Magn. Reson. Imaging, 2000. 12(4): p. 571-83.

51. Hindman, J., Proton resonance shift of water in the gas and liquid states. J. Chem. Phys., 1966. 44(12): p. 4582-4592.

52. Mills, J.K. and D. Needham, Lysolipid incorporation in dipalmitoylphosphatidylcholine bilayer membranes enhances the ion permeability and drug release rates at the membrane phase transition. Biochim. Biophys. Acta-Biomembr., 2005. 1716(2): p. 77-96.

53. Madden, T.D., et al., The accumulation of drugs within large unilamellar vesicles exhibiting a proton gradient-a survey. Chem. Phys. Lipids, 1990. 53(1): p. 37-46.

54. Li, X.G., et al., Doxorubicin physical state in solution and inside liposomes loaded via a pH gradient. Biochim. Biophys. Acta-Biomembr., 1998. 1415(1): p. 23-40.

55. Hossann, M., et al., In vitro stability and content release properties of phosphatidylglyceroglycerol containing thermosensitive liposomes. Biochim. Biophys. Acta-Biomembr., 2007. 1768(10): p. 2491-2499.

56. de Smet, M., et al., Magnetic resonance imaging of high intensity focused ultrasound mediated drug delivery from temperature-sensitive liposomes: an in vivo proof-of-concept study. J. Control. Release, 2010. 150(1): p. 102-10. 
57. Port, M., et al., Efficiency, thermodynamic and kinetic stability of marketed gadolinium chelates and their possible clinical consequences: a critical review. Biometals, 2008. 21(4): p. 469-490.

58. Kanal, E., et al., ACR Guidance Document for Safe MR Practices: 2007. Am. J. Roentgenol., 2007. 188(6): p. 1447-1474.

59. May, J.P. and S.-D. Li, Hyperthermia-induced drug targeting. Expert. Opin. Drug Deliv., 2013. 10(4): p. 511-527.

60. Al-Ahmady, Z. and K. Kostarelos, Chemical components for the design of temperature-responsive vesicles as cancer therapeutics. Chem. Rev., 2016. 116(6): p. 3883-3918.

61. Allen, T.M. and L.G. Cleland, Serum-induced leakage of liposome of contents. Biochim. Biophys. Acta, 1980. 597(2): p. 418-426.

62. Banno, B., et al., The Functional roles of poly(ethylene glycol)-lipid and lysolipid in the drug retention and release from lysolipid-containing thermosensitive liposomes in vitro and in vivo. J. Pharm. Sci., 2010. 99(5): p. 2295-2308.

63. Gasselhuber, A., et al., Mathematical spatio-temporal model of drug delivery from low temperature sensitive liposomes during radiofrequency tumour ablation. Int. J. Hyperth., 2010. 26(5): p. 499-513.

64. Sahani, D.V., et al., Assessing tumor perfusion and treatment response in rectal cancer with multisection CT: initial observations. Radiol., 2005. 234(3): p. 785-792. 


\section{Supplemental figures for chapter 2}

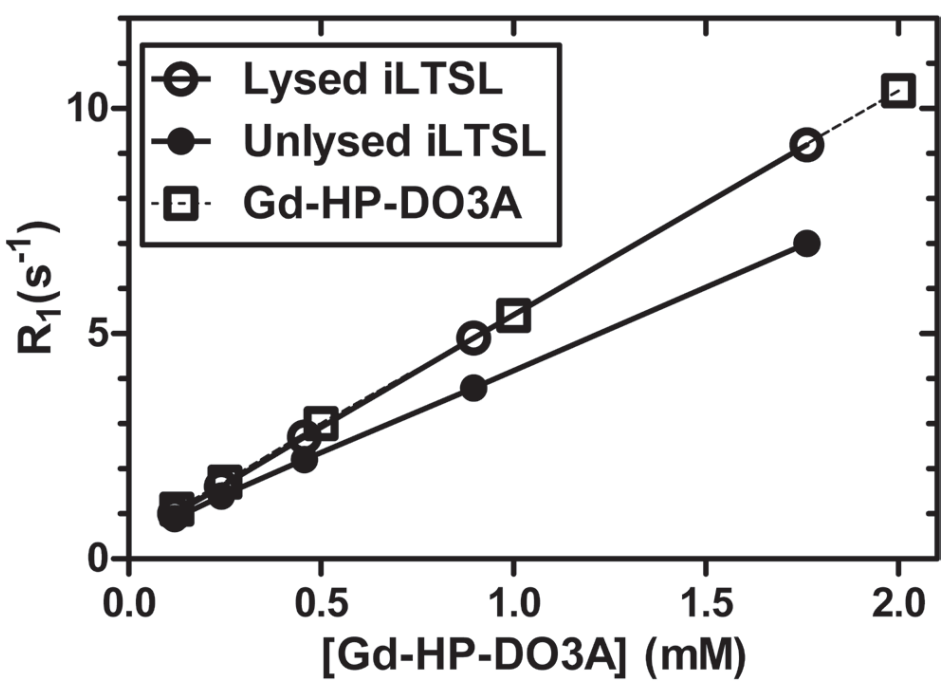

Supplemental Figure 1. Calibration of R1 vs. concentration of Gd-HP-DO3A at 0.5T. Triton $\mathrm{X}-100$ was added to lyse iLTSL, releasing Gd-HP-DO3A and the drug. The resulting relaxivity (slope) values for lysed and intact iLTSL were $4.994 \pm 0.010$ and $3.70 \pm 0.02 \mathrm{mM}^{-1} \mathrm{~s}^{-1}$, respectively, and were significantly different $(\mathrm{p}<0.0001, \mathrm{~F}$ test). Relaxivity of Gd-HP-DO3A $(4.96 \pm 0.02$ $\mathrm{mM}^{-1} \mathrm{~s}^{-1}$ ) was not significantly different from that of lysed iLTSL ( $\mathrm{p}=0.19$, F test). $\mathrm{R}^{2}>0.9999$ for all fitted data.
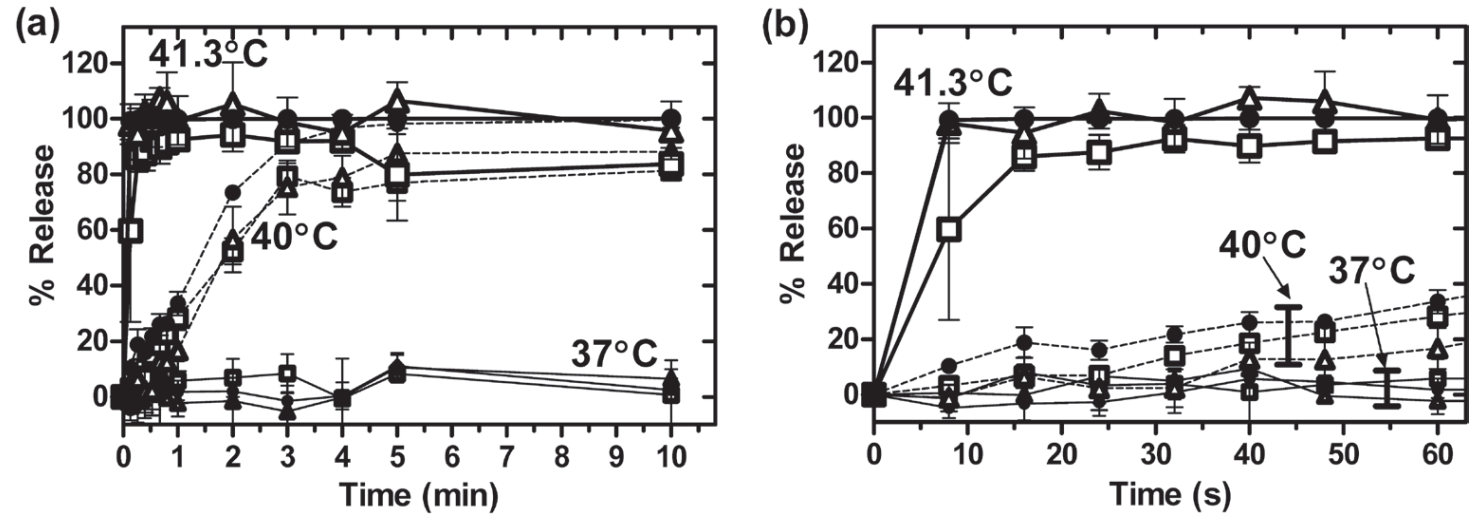

Supplemental Figure 2. Percent release of Gd-HP-DO3A at 37, 40 and $41.3{ }^{\circ} \mathrm{C}$. a) Release over 10 minutes. b) Release over first minute of heating. The methods used to approximate $\%$ Gd-HP-DO3A release were T1 measurements before (method 1, $\square$ ) and after (method 2, $\Delta$ ) passing aliquots through two size exclusion chromatography columns, as well as concentration measurements with ICP-AES (method 3, O). Symbol size indicates temperature: $37^{\circ} \mathrm{C}$ is smallest and $41.3{ }^{\circ} \mathrm{C}$ is largest. Maximum mean difference from ICP-AES measurements is $7 \pm 2 \%$ for $\square$ and $9 \pm 4 \%$ for $\Delta$. Percent release magnitudes were not statistically different between ICP-AES and the other two methods of measurements ( $p>0.05$, Dunn's multiple comparison). Each point represents the mean of 3 experiments \pm SEM. 


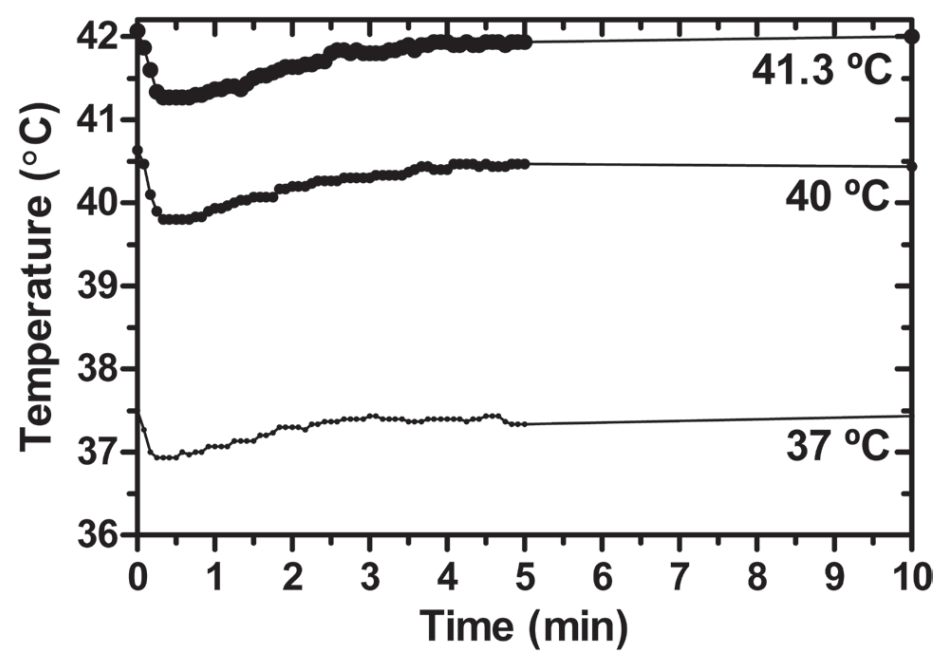

Supplemental Figure 3. Temperature during release assay at 37,40 and $41.3^{\circ} \mathrm{C}$ in which both doxorubicin and Gd-HP-DO3A release were quantified (corresponding to Figure 2). The initial decrease in temperature is due to the addition of concentrated liposomal solution to the preheated HEPES buffer. The temperature of each release assay (shown on the right) was the target minimum temperature reached.

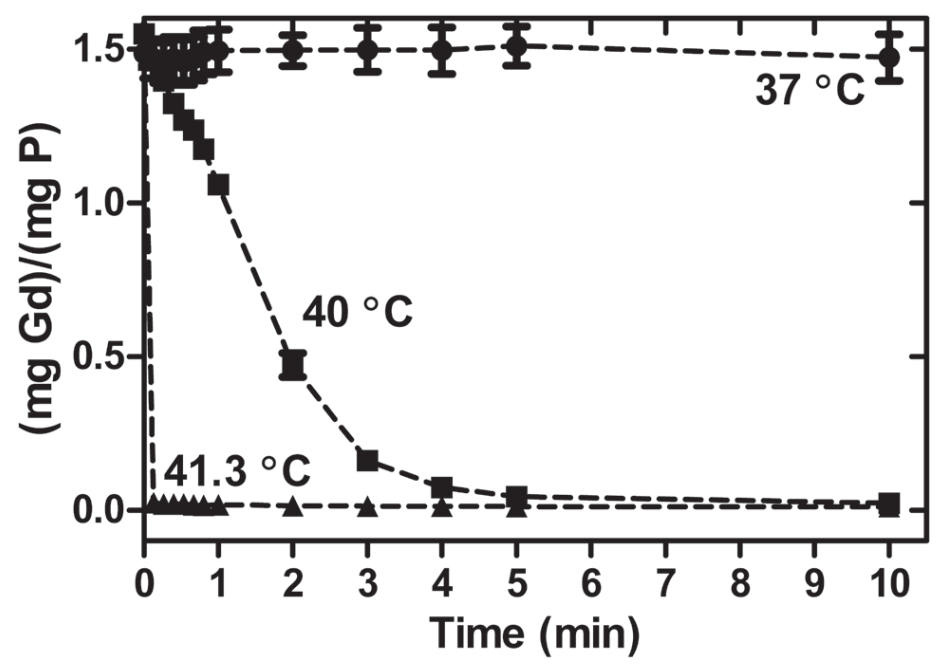

Supplemental Figure 4. Gd/P weight ratio as a function of time, as determined by ICP-AES at three temperatures, after released Gd-HP-DOA3 was removed with size exclusion chromatography $(\mathrm{n}=3)$. 


\section{Chapter 3}

\section{Synthesis and in vitro evaluation of novel cyclic NGR peptide targeted thermally sensitive liposome}

Ayele H. Negussie ${ }^{1}$, Jenna L. Miller ${ }^{1}$, Goutham Reddy ${ }^{1}$, Steven K. Drake ${ }^{2}$, Bradford J. Wood ${ }^{1}$, Matthew R. Dreher ${ }^{1}$

${ }^{1}$ Radiology and Imaging Sciences, Clinical Center, National Institutes of Health, Bethesda, MD, 20892, United States

${ }^{2}$ Critical Care Medicine Department, Clinical Center, National Institutes of Health, Bethesda, MD, 20892, United States

Published in: J. Control. Release., 143, 2010 


\section{Abstract}

The Asn-Gly-Arg (NGR) motif in both cyclic and linear form has previously been shown to specifically bind to CD13/aminopeptidase $\mathrm{N}$ that is selectively overexpressed in tumor vasculature and some tumor cells. However, previous versions of cyclic NGR used a liable disulfide bridge between cysteine residues that may be problematic for liposome targeting due to disulfide bond formation between adjacent peptides on the liposomal surface. In this study we report the design, synthesis, and characterization of a novel cyclic NGR containing peptide, cKNGRE, which does not contain a disulfide bridge. cKNGRE was synthesized in good yield and purity and attached to the fluorescent reporter Oregon Green (cKNGRE-OG) and lysolipid containing temperature sensitive liposomes (LTSLs). The identity of cKNGRE was verified with NMR and mass spectral techniques. In vitro fluorescence microscopy evaluation of cKNGRE-OG demonstrated binding and active uptake by $\mathrm{CD} 13^{+}$cancer cells and minimal binding to $\mathrm{CD}^{-} 3^{-}$cancer cells. The cKNGRE-OG ligand displayed 3.6-fold greater affinity for $\mathrm{CD}_{1} 3^{+}$cancer cells than a linear NGR-containing peptide. Affinity for $\mathrm{CD} 3^{+}$cancer cells was similarly improved 10 -fold for both the cyclic and linear NGR when presented in a multivalent fashion on the surface of an LTSL. cKNGRE-targeted LTSLs rapidly released ( $>75 \%$ in b4 s) doxorubicin at $41.3{ }^{\circ} \mathrm{C}$ with minimal release at $37^{\circ} \mathrm{C}$. These results demonstrate the ability to synthesize a cKNGRE-targeted temperature sensitive liposome that lacks a disulfide bridge and has sufficient binding affinity for biological applications. 


\section{Introduction}

The targeting of tumor-specific or tumor-associated antigens [1] is a widely used paradigm for broad applications including anticancer therapy, site-specific drug delivery, and molecular imaging. Often, these antigens are either entirely absent or barely present on normal cells and tissues [2]. The use of various tumorassociated antigens for targeted cancer therapies is well documented and includes leukocyte differentiation antigen (CD33) for acute myeloid leukemia [3], GD2 for neuroblastoma $[4,5]$, and the folate receptor for a wide variety of human tumors, especially ovarian carcinomas [6-8]. Antigens expressed on angiogenic tumor vasculature are particularly attractive tumor-associated targets because they have intimate contact with the blood and are therefore geographically accessible following intravenous injection of a targeting agent [9]. Widely used tumor vascular targets include integrins $[10,11]$, vascular endothelial growth factor receptor (VEGFR) [12, 13], platelet-derived growth factor receptor (PDGFR) $[14,15]$, and CD13/aminopeptidase N (APN, refer to as CD13 throughout) [2, 16-21]. CD13 is commonly expressed in angiogenic tumor vasculature and is the focus of this study.

Angiogenic tumor vessels are important elements for tumor growth and metastasis. They are essential for transporting metabolically important materials to and from the tumor cells and also provide a route for the dissemination of tumor cells to distal sites. The Asp-Gly-Arg (NGR) peptide motif has been used to target drugs and drug-containing liposomes to the tumor vascular antigen CD13, resulting in improved biodistribution and tumor therapy [21-24]. Although cyclic NGR peptides containing a disulfide bridge and linear NGR peptides have demonstrated suitable biodistribution and efficacy [21-28], the antitumor activity of drug associated with the cyclic form of NGR was reported to be 10-fold greater 
[22]. Despite the higher affinity of cyclic NGR peptides [22], there has been a preference to use linear NGR-containing motifs to target liposomes to avoid the formation of disulfide bridges between adjacent peptides on the liposome surface that may render the ligand ineffective [29].

The objectives of this study were to design and synthesize a novel cyclic NGR peptide that does not contain a disulfide bridge and to evaluate this peptide for specificity and affinity to $\mathrm{CD}_{13}{ }^{+}$cancer cells. A linear NGR control peptide was synthesized for comparison. Our long-term goal is to synthesize targeted lysolipid-containing temperature sensitive liposomes (LTSLs) for imageguided, heat-activated delivery of chemotherapeutics to solid tumors. LTSLs mainly composed of 1,2-dipalmitoyl-sn-glycero-3-phosphate (DPPC; $\mathrm{Tm}=$ $41.5^{\circ} \mathrm{C}$ ) [30] rapidly release their contents at clinically relevant hyperthermic temperatures $\left(40-42{ }^{\circ} \mathrm{C}\right)$ [31] when a small fraction $(-10 \%)$ of lysolipid (e.g., monostearoylphosphatidylcholine [MSPC]) is incorporated into the lipid bilayer [32]. LTSLs may be combined with focal hyperthermia or thermal ablation to selectively deliver encapsulated drugs to a heated region $[33,34]$. To this end, we have synthesized an NGR-targeted LTSL and evaluated the binding of the targeted LTSL to CD13 ${ }^{+}$cells. NGR-targeted LTSLs have the potential to improve therapeutic efficacy by: 1) slowing the transit time of liposomes in the tumor vasculature to improve drug release, 2) improving total drug accumulation in the tumor, and 3) treating metastatic tumors not subjected to hyperthermia.

\section{Materials and methods}

\section{Materials}

All reagents and solvents (anhydrous) were obtained from Sigma-Aldrich (MO) and used as received unless otherwise indicated. Fmoc-Arg(Pbf)-OH was supplied 
by either Novabiochem (CA) or Chem-Impex International Inc. (IL). NovaPEG Rink Amide Resin, HATU, and all other amino acids used in this study were purchased from Novabiochem. Fmoc-Gly-Rink-Amide MBHA Resin was purchased from Peptide International (KY). HOBt was purchased from AnaSpec (CA). Oregon Green 488 (OG) and 3, 3'-dioctadecyloxacarbocyanine perchlorate (DiO) were purchased from Invitrogen (OR). 1-stearoyl-2-hydroxy-sn-glycero-3phosphocholine (MSPC), 1,2-dipalmitoyl-sn-glycero-3-phosphocholine (DPPC), 2-distearoyl-sn-glycero-3-phosphoethanolamine-N-[carboxy(Polyethylene Glycol)2000] ammonium salt (DSPE- $\mathrm{PEG}_{2000} \mathrm{CH}_{2} \mathrm{COOH}$ ), and 1,2-distearoylsn-glycero-3-phosphoethanolamine- $\mathrm{N}$-[methoxy(polyethylene glycol)-2000] ammonium salt (DSPE-PEG ${ }_{2000}$ ) were obtained from Avanti Polar Lipids (AL). ${ }^{1} \mathrm{H}$ NMR and ${ }^{13} \mathrm{C}$ NMR spectra were recorded using Bruker 600 and $300 \mathrm{MHz}$ spectrometers operating at $600 \mathrm{MHz}$ for ${ }^{1} \mathrm{H}$ and $75 \mathrm{MHz}$ for ${ }^{13} \mathrm{C}$, respectively. Mass spectral data were recorded on PE/SCIEX API 2000 (Applied Biosystems Inc., CA) and UltraFlex TOF-TOF (Bruker Daltonics Inc., MA) instruments. Purification of peptides was performed using preparative RP-HPLC on a Varian ProStar model 330 PDA detector with a C-18 Microsorb column $(250 \times 21.4$ $\mathrm{mm}, 100 \AA / 5 \mu \mathrm{m})$. Analytical HPLC was performed using the same instrument and with a C-18 Microsorb column $(4.6 \times 250 \mathrm{~mm}, 100 \AA / 5 \mu \mathrm{m})$.

\section{Cell lines}

Human fibrosarcoma (HT-1080) and human adenocarcinoma (MCF7) cells were purchased from American Type Culture Collection (ATCC, VA). HT-1080 cells were grown in MEME (ATCC) supplemented with 10\% fetal bovine serum (ATCC), and MCF7 cells were grown in the same culture medium with the addition of $0.01 \mathrm{mg} / \mathrm{mL}$ bovine insulin (Sigma-Aldrich). Both cell lines were maintained in a $5 \% \mathrm{CO}_{2}$ incubator at $37^{\circ} \mathrm{C}$. Anti-CD13 monoclonal antibody (WM15, Alexa Fluor 488-conjugated) was obtained from AbD Serotec (NC). 


\section{Peptide synthesis}

\section{Cyclic KNGRE (cKNGRE) 3}

NovaPEG Rink Amide Resin 1 (0.50-0.80 mmol/g loading) was washed with dichloromethane (DCM) and 1-methyl-2-pyrrolidinone (NMP) and swollen with DCM for $2 \mathrm{~h}$. The resin was then washed with NMP and coupled with glutamic acid-via its $\mathrm{C} \alpha$-carboxylic acid-by agitating the resin with a solution of Fmoc-Glu(OAll)-OH (2.5 equiv), HATU (2 equiv), and DIPEA (4 equiv) in NMP. The resin was capped by washing with a solution of $\mathrm{CH}_{2} \mathrm{Cl}_{2}$-Acetic anhydride-DIPEA (20:4:1). The Fmoc protecting group was removed by treating the resin-attached peptide with a piperidine (20\%) in NMP for 5 min (2 times). The linear precursor peptides were constructed using Fmoc chemistry by adding the respective protected amino acid ( 2 equiv), HATU ( 2 equiv), and DIPEA ( 4 equiv) in NMP to give the linear pentapeptide-resin 2 . The $\mathrm{C} \omega$-terminal allyl ester of Glu was removed by treating the resulting penta-peptide with $\mathrm{Pd}(\mathrm{PPh} 3) 4(2$ equiv) in $\mathrm{CHCl} 3-\mathrm{AcOH}-\mathrm{N}$-methylmorpholine (37:2:1) under argon atmosphere by gentle shaking for $2 \mathrm{~h}$ and then washing with DIPEA-NMP (5:95) followed by $0.5 \%(\mathrm{w} / \mathrm{v})$ of sodium diethyldithiocarbamate trihydrate in NMP (2 times $2 \mathrm{~min}$ a total of $100 \mathrm{~mL}$ ). On-resin cyclization was performed by removing the $\mathrm{N}$-Fmoc group from the $\alpha$-amine of Lys and activating the $\mathrm{C} \alpha$-carboxylic acid of Glu with HATU (2 equiv) and DIPEA (4 equiv). Cleavage of the peptide from the resin and removal of all the protecting groups was performed by agitating the resin-peptide with TFA-DCM (1:1) for $2 \mathrm{~h}$ and washing with TFA-DCM (1:9). The acid wash was concentrated and $\mathrm{Et} 2 \mathrm{O}$ was added until a white precipitate separated. The precipitate was freed from the solvent, dissolved in water, purified by preparative RP-HPLC using a gradient of $\mathrm{MeCN}_{-} \mathrm{H}_{2} \mathrm{O}$ (5-30\% containing $0.1 \% \mathrm{TFA}, 10 \mathrm{~mL} / \mathrm{min}, 20 \mathrm{~min}$ run time), and lyophilized to give compound 3 as a white powder (193 mg, 33\% based on estimated loading of peptide-resin). ${ }^{1} \mathrm{H}$ NMR $\left(600 \mathrm{MHz}, \mathrm{D}_{2} \mathrm{O}\right): \delta=1.45-1.54(\mathrm{~m}, 2 \mathrm{H}), 1.57-1.81(\mathrm{~m}, 8 \mathrm{H}), 2.04-$ 
$2.10(\mathrm{~m}, 2 \mathrm{H}), 2.17-2.23(\mathrm{~m}, 1 \mathrm{H}), 2.36-2.41(\mathrm{~m}, 1 \mathrm{H}), 2.78-2.80(\mathrm{dd}, 1 \mathrm{H}, \mathrm{J}=$ 7.8, 17.7 Hz), 2.83-2.87 (m, 1H), 3.05-3.09 (dd, $1 \mathrm{H}, \mathrm{J}=4.8,15.6 \mathrm{~Hz}), 3.22$ $(\mathrm{t}, 2 \mathrm{H}, \mathrm{J}=7.0 \mathrm{~Hz}), 3.9(\mathrm{~d}, 1 \mathrm{H}, \mathrm{J}=17.7 \mathrm{~Hz}), 4.14-4.23(\mathrm{~m}, 3 \mathrm{H}), 4.46-4.48(\mathrm{~m}$, $2 \mathrm{H}) .{ }^{13} \mathrm{C}$ NMR $\left(75 \mathrm{MHz}, \mathrm{D}_{2} \mathrm{O}\right) \delta=22.3,23.8,24.6,26.4,27.9,30.5,31.5$, 34.5, 39.1, 40.4, 42.9, 51.3, 52.8, 54.5, 55.5, 156.7, 172.4, 172.7, 174.0, 175.3, 175.3, 175.8, 176.2. Theoretical mass calculated for cKNGRE $\left(\mathrm{C}_{23} \mathrm{H}_{41} \mathrm{~N}_{11} \mathrm{O}_{7}\right)$ was 583.319; found MALDI-TOF-MS: m/z 584.24 [M+H] ${ }^{+}$, ESI-MS: m/z 584.3 $[\mathrm{M}+\mathrm{H}]^{+}$. Analytical HPLC revealed a purity of $98 \%$ at $210 \mathrm{~nm}, \mathrm{t}_{\mathrm{R}}=10.05 \mathrm{~min}$, using a gradient of $\mathrm{MeCN}-\mathrm{H}_{2} \mathrm{O}(5-30 \%$ containing $0.1 \%$ TFA, $1 \mathrm{~mL} / \mathrm{min}, 20$ min run time).

\section{Linear $K N G R G(K N G R G) \mathbf{4}$}

Synthesized using the same protocol as described above except Gly preloaded Rink amid MBHA resin was used instead of Glu to avoid the accompanying reactive functional group. After assembling the last amino acid, the Fmoc group was removed, the $\alpha$-amine of Lys was acetylated, and the linear peptide was cleaved from the resin as described above. The peptide was then purified with preparative

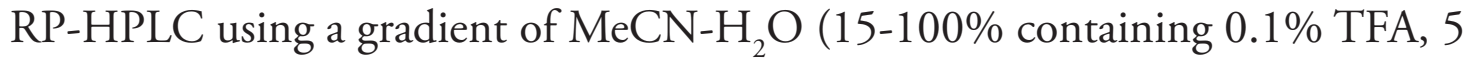
$\mathrm{mL} / \mathrm{min}, 20 \mathrm{~min}$ run time) and lyophilized to give compound $\mathbf{4}$ as a white powder (197 mg, 34.5 \%). ${ }^{1} \mathrm{H}$ NMR (600 MHz, $\left.\mathrm{D}_{2} \mathrm{O}\right) \delta=1.39-1.50(\mathrm{~m}, 2 \mathrm{H}), 1.60-1.94$ $(\mathrm{m}, 8 \mathrm{H}), 2.04(\mathrm{~s}, 3 \mathrm{H}, \mathrm{Me}), 2.79(\mathrm{dd}, 1 \mathrm{H}, \mathrm{J}=8.0,15.7 \mathrm{~Hz}), 2.88(\mathrm{dd}, 1 \mathrm{H}, \mathrm{J}=$ 5.6, $15.7 \mathrm{~Hz}), 2.99(\mathrm{t}, 2 \mathrm{H}, \mathrm{J}=7.6 \mathrm{~Hz}), 3.22(\mathrm{t}, 2 \mathrm{H}, \mathrm{J}=7.0 \mathrm{~Hz}), 3.9(\mathrm{~d}, 2 \mathrm{H}, \mathrm{J}=$ $6.5 \mathrm{~Hz}), 3.97(\mathrm{~s}, 2 \mathrm{H}), 4.25(\mathrm{dd}, 1 \mathrm{H}, \mathrm{J}=5.6,8.8 \mathrm{~Hz}), 4.36(\mathrm{dd}, 1 \mathrm{H}, \mathrm{J}=5.3,8.9$ $\mathrm{Hz}), 4.72(\mathrm{dd}, 1 \mathrm{H}, \mathrm{J}=5.6,7.9 \mathrm{~Hz}) .{ }^{13} \mathrm{C} \mathrm{NMR}\left(75 \mathrm{MHz}, \mathrm{D}_{2} \mathrm{O}\right) \delta=21.7,22.0$, $24.3,26.2,27.8,30.1,35.9,39.2,40.5,42.1,42.6,50.5,53.6,54.0,156.8$, 171.6, 173.0, 174.0, 174.1, 174.3, 174.6, 174.8. Theoretical mass calculated for KNGRG $\left(\mathrm{C}_{22} \mathrm{H}_{41} \mathrm{~N}_{11} \mathrm{O}_{7}\right)$ was 571.319; found MALDI-TOF-MS: m/z 572.21 $[\mathrm{M}+\mathrm{H}]^{+}$, ESI-MS: $572.3[\mathrm{M}+\mathrm{H}]^{+}$. Analytical HPLC revealed a purity of $99 \%$ at 
$210 \mathrm{~nm}, \mathrm{t}_{\mathrm{R}}=6.85 \mathrm{~min}$, using a gradient of $\mathrm{MeCN}-\mathrm{H}_{2} \mathrm{O}(15-100 \%$ containing $0.1 \%$ TFA, $0.5 \mathrm{~mL} / \mathrm{min}, 10 \mathrm{~min}$ run time).

\section{Conjugation of peptides to Oregon Green}

General procedure:

DIPEA $(3 \mu \mathrm{L})$ was added to a solution of NGR peptide (1.0 equiv) and Oregon Green 488 carboxylic acid, succinimidyl ester, 6-isomer $(5 \mathrm{mg}, 0.0098 \mathrm{mmol}$, 1.1 equiv) in NMP and the resulting reaction mixture was stirred for $5 \mathrm{~h}$ at room temperature. The reaction mixture was precipitated by pouring it into $20 \mathrm{~mL}$ of diethyl ether and then filtering and washing it with diethyl ether. The resulting ether-free precipitate was dissolved in water and purified with preparative RPHPLC.

\section{cKNGRE-OG (5a)}

Purified by preparative HPLC using a gradient of $\mathrm{MeCN}_{-} \mathrm{H}_{2} \mathrm{O}(20-40 \%$ containing $0.1 \%$ TFA, $5 \mathrm{~mL} / \mathrm{min}, 30 \mathrm{~min}$ run time) and lyophilized to yield the desired Oregon Green coupled peptide 5a as a yellow powder $(6.3 \mathrm{mg}, 72 \%) .{ }^{1} \mathrm{H}$ $\operatorname{NMR}\left(600 \mathrm{MHz}, \mathrm{D}_{2} \mathrm{O}\right): \delta=1.31-1.64(\mathrm{~m}, 10 \mathrm{H}), 1.88-2.05(\mathrm{~m}, 3 \mathrm{H}), 2.23(\mathrm{~m}$, 1H), $2.53(\mathrm{~m}, 1 \mathrm{H}), 2.72(\mathrm{~m}, 1 \mathrm{H}), 2.95(\mathrm{~m}, 1 \mathrm{H}), 3.11(\mathrm{t}, 2 \mathrm{H}, \mathrm{J}=6.8 \mathrm{~Hz}), 3.21$ $(\mathrm{m}, 2 \mathrm{H}), 4.15(\mathrm{~d}, 1 \mathrm{H}, \mathrm{J}=17.6 \mathrm{~Hz}), 4.34-4.37(\mathrm{~m}, 1 \mathrm{H}), 4.41-4.42(\mathrm{~m}, 1 \mathrm{H}), 6.56$ (s, 2H), 6.74 (s, 2H), $7.58(\mathrm{~s}, 1 \mathrm{H}), 7.97(\mathrm{~d}, \mathrm{~J}=8.0 \mathrm{~Hz}, 1 \mathrm{H}), 8.12(\mathrm{~d}, \mathrm{~J}=8.0$ $\mathrm{Hz}, 1 \mathrm{H})$. Theoretical mass calculated for cKNGRE-OG $\left(\mathrm{C}_{44} \mathrm{H}_{49} \mathrm{~F}_{2} \mathrm{~N}_{11} \mathrm{O}_{13}\right)$ was 977.348; found MALDI-TOF-MS: m/z 978.36 (M +H) ${ }^{+}$; ESI-MS: m/z 978.3 $[\mathrm{M}+\mathrm{H}]^{+}$. Purity was determined by analytical HPLC to be $99.5 \%$ at $254 \mathrm{~nm}, \mathrm{t}_{\mathrm{R}}$ $=5.39 \mathrm{~min}$, using a gradient of $\mathrm{MeCN}_{-} \mathrm{H}_{2} \mathrm{O}(30-40 \%$ containing $0.1 \% \mathrm{TFA}, 1$ $\mathrm{mL} / \mathrm{min}, 15 \mathrm{~min}$ run time). 
$K N G R G-O G(\mathbf{5 b})$

Purified by preparative HPLC using a gradient of $\mathrm{MeCN}_{-} \mathrm{H}_{2} \mathrm{O}(30-80 \%$ containing $0.1 \%$ TFA, $5 \mathrm{~mL} / \mathrm{min}, 25 \mathrm{~min}$ run time) and lyophilized to give the desired Oregon Green coupled peptide $\mathbf{5 b}$ as a yellow powder (6.5 mg, 67.5\%). Theoretical mass calculated for KNGRG-OG $\left(\mathrm{C}_{43} \mathrm{H}_{49} \mathrm{~F}_{2} \mathrm{~N}_{11} \mathrm{O}_{13}\right)$ was 965.348; found MALDI-TOF-MS: m/z $966.28[\mathrm{M}+\mathrm{H}]^{+}$; ESI-MS: m/z 988.2 [M+Na] $]^{+}$, $966.0[\mathrm{M}+\mathrm{H}]^{+}$. Analytical HPLC revealed a purity of $98.5 \%$ at $254 \mathrm{~nm}, \mathrm{t}_{\mathrm{R}}=7.04$ min, using a gradient of $\mathrm{MeCN}-\mathrm{H}_{2} \mathrm{O}(30-100 \%$ containing $0.1 \%$ TFA, $0.5 \mathrm{~mL} /$ min, 10 min run time).

\section{Coupling of the peptides onto DSPE-PEG ${ }_{2000} \mathrm{CH}_{2} \mathrm{COOH}$}

General Procedure

Average MW of DSPE-PEG ${ }_{2000} \mathrm{CH}_{2} \mathrm{COOH}$ was $2788.84 \pm 44 \mathrm{n}(\mathrm{n}=0-8) \mathrm{g} / \mathrm{mol}$. To a solution of DSPE-PEG ${ }_{2000} \mathrm{CH}_{2} \mathrm{COOH}(50 \mathrm{mg}, 0.018 \mathrm{mmol}), \mathrm{DCC}$ (4.4 $\mathrm{mg}, 0.021 \mathrm{mmol})$, and HOBt $(2.8 \mathrm{mg}, 0.021 \mathrm{mmol})$ in NMP; DIPEA $(10 \mu \mathrm{L})$ was added and stirred for $30 \mathrm{~min}$ at room temperature. Peptide 3 or 4 (1.2 equiv.) was then added, and the resulting reaction mixture was allowed to stir overnight at ambient temperature. The mixture was powderized by pouring into diethyl ether $(20 \mathrm{~mL})$, and the precipitate was washed with diethyl ether and dried. The dried powder was dissolved with $\mathrm{MeOH}: \mathrm{CHCl}_{3}$ (1:2) and purified with Sephadex-LH20 $\left(\mathrm{CHCl}_{3}: \mathrm{MeOH}, 2: 1\right)$ to give DSPE-PEG ${ }_{2000} \mathrm{CH}_{2} \mathrm{CO}$-peptide as a transparent solid after evaporation of the solvent.

\section{DSPE-PEG ${ }_{2000} \mathrm{CH}_{2} \mathrm{CO}-\mathrm{cKNGRE} \mathbf{6 a}$}

Theoretical mass calculated for $\mathrm{C}_{157} \mathrm{H}_{303} \mathrm{~N}_{12} \mathrm{O}_{63} \mathrm{P}$ was 3396.07 (average); found MALDI-TOF-MS: $\mathrm{m} / \mathrm{z} 3397.06 \pm 44 \mathrm{n}(\mathrm{n}=0-8)[\mathrm{M}+\mathrm{H}]^{+}$. Yield was $(40 \mathrm{mg}$, $65 \%)$. 


\section{DSPE-PEG ${ }_{2000} \mathrm{CH}_{2} \mathrm{CO}-\mathrm{KNGRG}$ 6b:}

Theoretical mass calculated for $\mathrm{C}_{156} \mathrm{H}_{303} \mathrm{~N}_{12} \mathrm{O}_{63} \mathrm{P}$ was 3385.06 (average); found MALDI-TOF-MS: $\mathrm{m} / \mathrm{z} 3385.36 \pm 44 \mathrm{n}(\mathrm{n}=0-8)[\mathrm{M}+\mathrm{H}]{ }^{+}$. Yield was $48.8 \mathrm{mg}$, $80 \%)$.

\section{Liposome preparation}

\section{NGR-targeted liposomes}

Fluorescently labeled NGR-targeted liposomes were prepared as follows. DPPC: MSPC: DSPE-PEG2000-NGR: DiO in molar percent ratio of 85.2: 9.7: 5: 0.1 were dissolved in chloroform, mixed, dried by solvent evaporation, and left overnight in a vacuum desiccator. The dried film was hydrated with $2.5 \mathrm{~mL}$ of HEPES buffer ( $\mathrm{pH} 7.4,10 \mathrm{mM}$ HEPES, $140 \mathrm{mM} \mathrm{NaCl}, 280 \mathrm{mOsm}$ ) at $55^{\circ} \mathrm{C}$ for $1 \mathrm{~h}$ to yield a final lipid concentration of $10 \mathrm{mg} / \mathrm{mL}$. The resulting multilamellar preparation was sized by by extrusion (10 times) with a LIPEXTM Extruder (Northern Lipids Inc., Canada) at $55^{\circ} \mathrm{C}$ through two stacked NucleporeÒ polycarbonate membrane filters (Whatman plc, United Kingdom) with a pore size of $100 \mathrm{~nm}$. The particle size of the liposome was determined by dynamic light scattering (ZetaPALS, Brookhaven Instruments Corporation, NY) and reported as the mean diameter \pm one standard deviation. $\mathrm{DiO}$ was included to monitor the liposome by this fluorescent label with flow cytometry.

\section{Doxorubicin encapsulation}

Dox loaded NGR-targeted liposomes were prepared as follows. DPPC:MSPC:DSPE-PEG2000-cNGR in molar percent ratio of 85.3:9.7:5 were prepared as described above. The dried film was hydrated with $300 \mathrm{mM}$ citric acid ( $\mathrm{pH} 4.0$ ) at $60^{\circ} \mathrm{C}$ for $15 \mathrm{~min}$ to yield a final lipid concentration of $50 \mathrm{mg} / \mathrm{mL}$. The resulting multilamellar preparation was sized and its particle size was determined as described above. Encapsulation of Dox into the extruded liposomes was carried 
out using the $\mathrm{pH}$ gradient loading protocol as described by Mayer et al. [35] with slight modification. Briefly, the exterior $\mathrm{pH}$ of the extruded liposomes was titrated to 7.4 with sodium carbonate solution $(500 \mathrm{mM})$ creating a $\mathrm{pH}$ gradient (acidic inside). The liposomes were incubated with Dox (at Dox: lipid wt. ratio of 5:100) at $37^{\circ} \mathrm{C}$ for $1 \mathrm{~h}$ and passed through Sephadex-G50 (fine) spin column. Liposome entrapped Dox was determined using UV-Vis spectrophotometer [36]. Dox loading efficiency is consistently $>98 \%$ for LTSLs using this method.

\section{Temperature triggered release of Dox from cNGR-LTSLs in vitro}

The release of encapsulated Dox from cNGR-LTSLs as a function of temperature $\left(25\right.$, and $\left.37-41.3^{\circ} \mathrm{C}\right)$ was determined by measuring the dequenching of Dox fluorescence as it was released from a liposome over a period of 15 min using Cary Eclipse spectrofluorometer equipped with Eclipse multicell peltier, temperature controller, and Eclipse Kinetic Software (Varian Inc.) at an excitation and emission wavelength of 498 and $593 \mathrm{~nm}$, respectively. A 10- $\mu \mathrm{L}$ sample of liposome was added into a cuvette containing $2 \mathrm{~mL}$ of HEPES buffer equilibrated to the desired temperature and the fluorescent intensity was measured at $2 \mathrm{~s}$ intervals for the first $300 s$ and $5 s$ interval for the remainder. Then Triton ${ }^{\oplus} \mathrm{X}-100(10 \mu \mathrm{L}, 10 \%$ $\mathrm{w} / \mathrm{w}$ in $0.9 \%$ saline) was added to completely disrupt the liposomal bilayer for complete release of the entrapped Dox. Percent release is calculated by assuming $100 \%$ release with Triton ${ }^{\circledR} \mathrm{X}-100$ and $0 \%$ release at $25{ }^{\circ} \mathrm{C}$ in a HEPES buffer. Data are presented as the mean percent release $(n=3)$.

\section{In vitro imaging studies}

Cellular binding of the linear and cyclic forms of NGR-OG to CD13 was assessed by plating HT-1080 (CD13 $\left.{ }^{+}\right)$and MCF7 (CD13) cells in eightchambered slides (LabTekII, Nunc, NY) at a concentration of 15,000 cells/well. After $24 \mathrm{~h}$, cells were washed once with phosphate-buffered saline (PBS) and 
incubated in complete culture media at $37^{\circ} \mathrm{C}$ for $30 \mathrm{~min}$ with cKNGRE-OG $(20 \mu \mathrm{M})$, KNGRG-OG $(20 \mu \mathrm{M})$, or a 1:20 dilution of anti-CD13 antibody (WM15, Alexa Fluor 488-conjugated, obtained from AbD Serotec, NC, final concentration $2.5 \mu \mathrm{g} / \mathrm{mL}$ ). Slides were washed twice with PBS for $5 \mathrm{~min}$, fixed in 4\% paraformaldehyde for $20 \mathrm{~min}$, mounted with Prolong Gold antifade reagent with DAPI (Invitrogen), and imaged with an epifluorescent microscope (Zeiss, Axio Imager. M1, NY). This dose of NGR $(20 \mu \mathrm{M})$ provided the best contrast between CD13 positive and negative cells for a range of examined doses $(5,10$, $20,40,60$, and $80 \mu \mathrm{M})$.

To examine internalization, cells were incubated with $20 \mu \mathrm{M}$ cKNGRE-OG in complete culture media for $30 \mathrm{~min}$ at $37^{\circ} \mathrm{C}$ or $4^{\circ} \mathrm{C}$, washed twice with PBS, and placed in complete media for $30 \mathrm{~min}$ at $37^{\circ} \mathrm{C}$. slides were fixed and mounted as above. Cells were imaged using the same methods as above for epifluorescent microscopy or with a LSM-710 laser scanning confocal fluorescence microscope (Zeiss) using a $63 \mathrm{x}$ objective. Emission and excitation spectra were set to manufacture's recommended settings for all fluorophores.

\section{In vitro binding studies}

The influence of cyclization of the NGR peptide on its affinity for both the free peptide and the peptide conjugated to an LTSL was studied by a FACSCalibur flow cytometer (Becton Dickinson, Ca) with a $488 \mathrm{~nm}$ laser. Samples were read on the FITC channel and analyzed using Cell Quest Pro Software on a Mac G3. CD13+ HT-1080 cells were incubated with cKNGRE-OG, KNGRG-OG, DiO-modified cKNGRE-LTSL, or DiO-modified KNGRG-LTSL at final NGR concentrations of 400, 200,100, 50, 25, 12.5, 6.25, 3.125, 1.625, 0.75, and $0.375 \mu \mathrm{M}$. The anti-CD13 antibody was used as a positive control. Fluorescence, for gated cell populations, was analyzed for 15,000 events (counts) per sample 
with consistent gating for the free peptide group and for the LTSL group. The $\%$ positive cells were fit with the Hill equation to determine the half maximal effective concentration $\left(\mathrm{EC}_{50}\right)$ for binding.

\section{Results}

\section{NGR peptide synthesis}

The cKNGRE peptide was assembled using manual solid phase Fmoc peptide chemistry [37] as outlined in Figure 3.1. N-Fmoc-Glu(OAll)-OH was selected as an anchoring site for the cyclization using its $\omega$-carboxylic acid. HATU, 2 equiv, based on amino acids was used for peptide coupling in N-Methyl-2pyrrolidinone. Piperidine in NMP was used for deprotection of the Fmoc group of the $\mathrm{N}$-terminus and orthogonal deprotection of the $\mathrm{C} \omega$-allyl ester of the Glu using $\mathrm{Pd}\left(\mathrm{PPh}_{3}\right)_{4}$ in $\mathrm{CHCl} 3-\mathrm{AcOH}-\mathrm{N}-$ Methylmorpholine [38]. On the resin cyclization

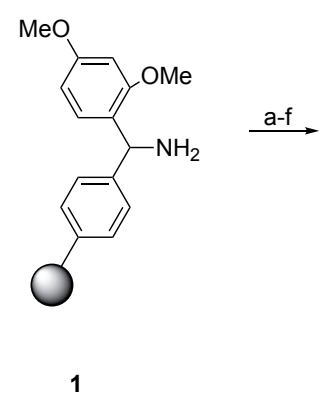

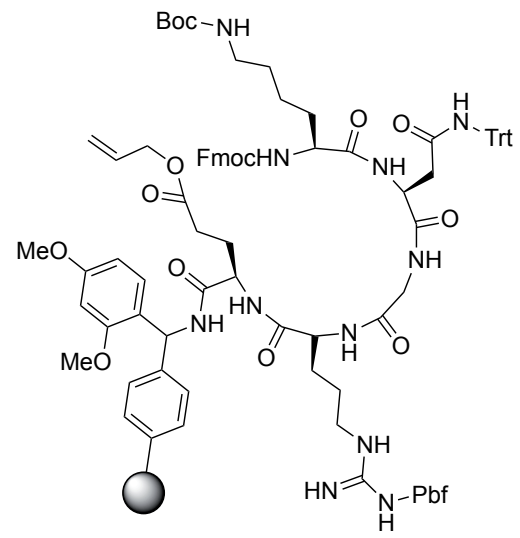

2

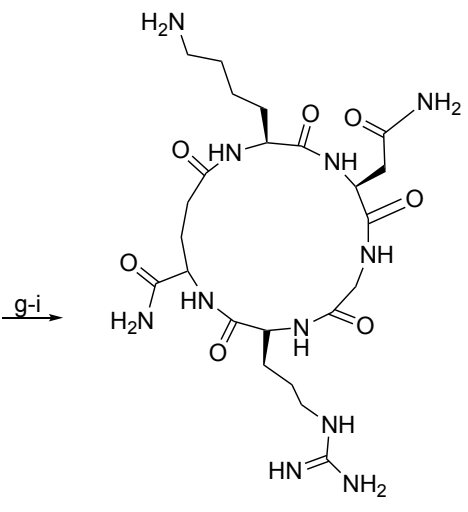

3

Figure 3.1 Reagents and conditions: a) N-Fmoc-Glu-(OAll)-OH (2.5 equiv), HATU (2 equiv), DIPEA (4 equiv), NMP, rt, 30 min.; b) Piperidine-DMF (1:4), rt, 5 min (X 2); c) N-FmocArg-(Pbf)-OH (2 equiv), HATU, (2 equiv), DIPEA (4 equiv), rt, $30 \mathrm{~min}$; d) N-Fmoc-Gly-OH, HATU, (2 equiv), DIPEA (4 equiv), rt, 30 min; e) N-Fmoc-Asn-(Trt)-OH, HATU, (2 equiv), DIPEA (4 equiv), rt, 30 min; f) N-Fmoc-Lys-(Boc)-OH, HATU, (2 equiv), DIPEA (4 equiv), rt, 30 min; g) Pd(PPh3)4 (2 equiv), $\mathrm{CHCl}_{3}-\mathrm{AcOH}_{-} \mathrm{N}$ - Methylmorpholine (37:2:1), rt, $2 \mathrm{~h}$; h) HATU, (2 equiv), DIPEA (4 equiv), rt, $30 \mathrm{~min} ; 17 \mathrm{~h}$; i) TFA- $\mathrm{CH}_{2} \mathrm{Cl}_{2}(1: 1), \mathrm{rt}, 2 \mathrm{~h}$. 
was carried out by coupling the $\omega$-carboxylic acid of the Glu to the $\alpha$-amine of the Lys with HATU at high dilution [37]. Removal of the cyclic peptide from the resin and deprotection was performed using TFA in dichloromethane. The crude peptide was concentrated and purified by preparative RP-HPLC on a C18 column, obtaining 3 as a white powder that was 98\% pure and had 33\% yield.

The linear KNGRG 4 was synthesized using the same methodology except FmocGly preloaded Rink Amide Resin (Figure 3.2) was used instead of Glu to avoid the side chain reactive functional group. The overall product yield obtained was $34.5 \%$ with a purity of $99 \%$. The free amines of the Lys residues of 3 and $\mathbf{4}$ were used to introduce the fluorescent label OG (1.1 equiv) to obtain compounds $\mathbf{5 a}$ and $\mathbf{5 b}$, respectively (Figure 3.3). Compound $\mathbf{5 a}$ and $\mathbf{5 b}$ were purified with preparative reverse phase HPLC using a C18 column to give a yellow powder in 72 and $67.5 \%$ yield, respectively.

\section{NGR targeted LTSL synthesis}

NGR peptides were conjugated to DSPE-PEG2000CH2COOH to give compounds $\mathbf{6 a}$ and $\mathbf{6 b}$ (Figure 3.4). In both cases the progress of the reaction was monitored by MALDI-TOF-MS until almost no DSPE-PEG2000CH2COOH

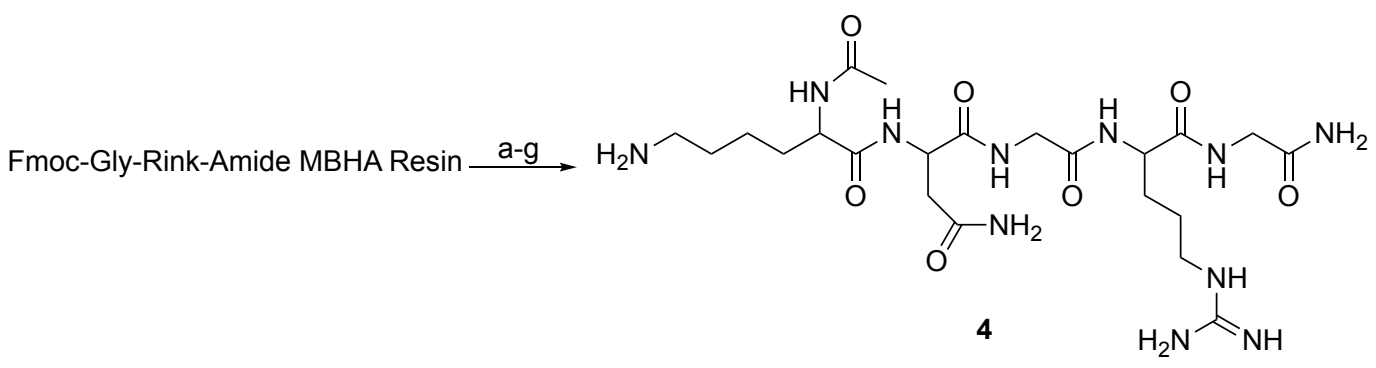

Figure 3.2 Reagents and conditions: a) Piperidine-DMF (1:4), rt, $5 \mathrm{~min}$ (X 2); b) N-FmocArg-(Pbf)-OH (2 equiv), HATU, (2 equiv), DIPEA (4 equiv), rt, $30 \mathrm{~min}$; c) N-Fmoc-Gly-OH, HATU, (2 equiv), DIPEA (4 equiv), rt, $30 \mathrm{~min}$; d) N-Fmoc-Asn-(Trt)-OH, HATU, (2 equiv), DIPEA (4 equiv), rt, 30 min; e) N-Fmoc-Lys-(Boc)-OH, HATU, (2 equiv), DIPEA (4 equiv), rt, 30 min; f) $\mathrm{CH}_{2} \mathrm{Cl}_{2}$ : Ac2O: DIPEA (20: 40: 1); g) TFA- $\mathrm{CH}_{2} \mathrm{Cl}_{2}(1: 1)$, rt, 2 h. 
was observed. Compounds $\mathbf{6 a}$ and $\mathbf{6 b}$ were purified by size exclusion chromatography using Sephadex LH-20 that was swollen in and eluted with $\mathrm{CCl}_{3} \mathrm{H}: \mathrm{MeOH}(2: 1)$. Peaks of the expected $\mathrm{m} / \mathrm{z}$ ratios for compounds $6 \mathrm{a}(65 \%$ yield) and $\mathbf{6 b}$ (80\% yield) were confirmed by MALDI-TOF-MS experiments (Figure 3.5). Minor peaks consistent with sodium salts of $6 a$ and $6 b(m / z+22)$ are seen between the major peaks in both spectra. NGR-targeted LTSLs were

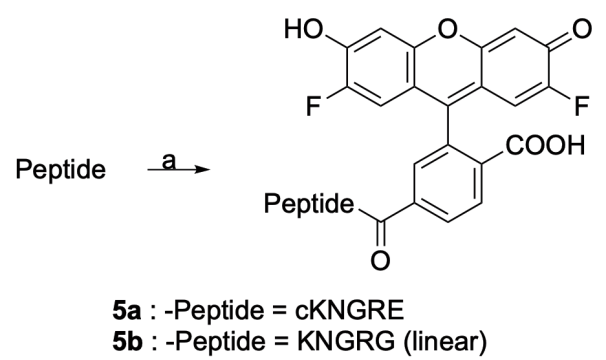

Figure 3.3 Reagents and conditions: a) Oregon Green 488 carboxylic acid, succinimidyl ester, 6-isomer (1.1 equiv), DIPEA (2 equiv), NMP ( $1 \mathrm{~mL}), \mathrm{rt}, 5 \mathrm{hr}$.

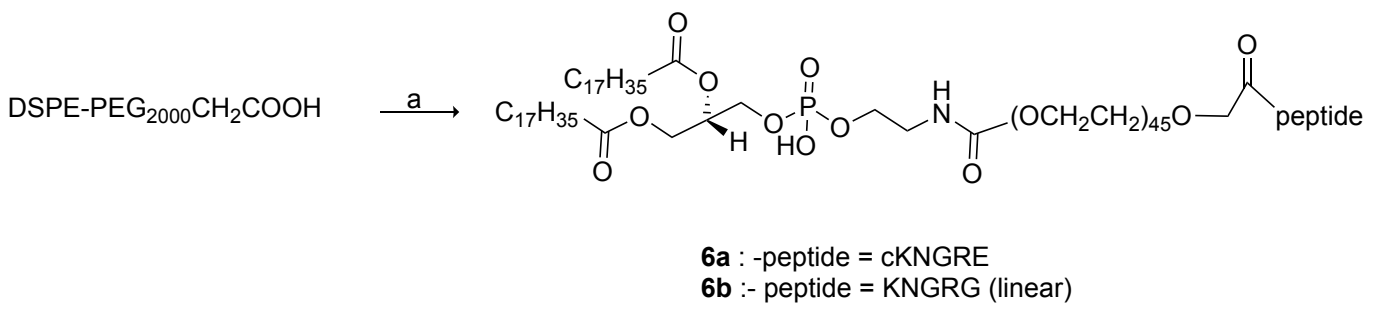

Figure 3.4 Reagents and conditions: a) HOBt (1.1 equiv), DCC (1.1 equiv.), DIPEA (3 equiv), NMP (1 mL), 30 min., 3 or 4 (1.2 equiv), rt, overnight.
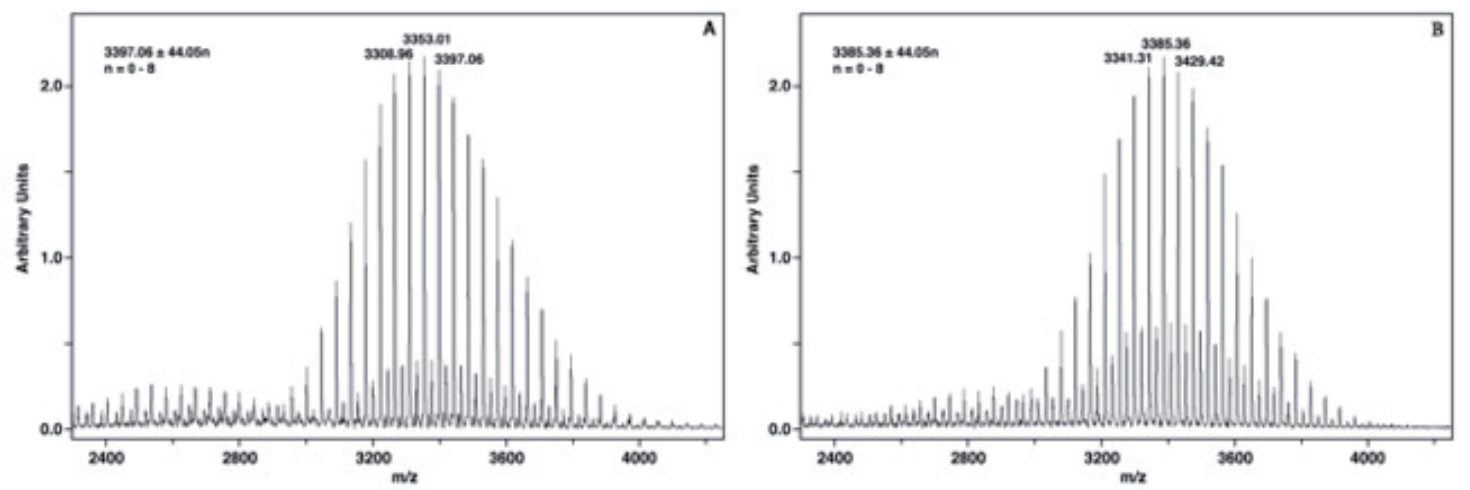

Figure 3.5 MALDI-TOF-MS spectra for compounds $6 \mathrm{a}(\mathrm{A})$ and $6 \mathrm{~b}(\mathrm{~B})$ 
synthesized with a particle size of $99.0 \pm 0.4 \mathrm{~nm}$ for KNGRG-LTSL and 105.6 $\pm 1.1 \mathrm{~nm}$ for cKNGRE-LTSL. Dox encapsulated cKNGRE-targeted LTSLs liposome had a particle size of $107.8 \pm 1.5 \mathrm{~nm}$.

\section{In vitro binding of NGR peptide and NGR targeted LTSL to CD13}

To evaluate in vitro binding of NGR peptide to human carcinoma cells, cKNGRE was conjugated to the fluorophore OG for visualization via epifluorescent microscopy. cKNGRE-OG and the anti-CD13 antibody, WM15, were incubated with HT-1080 (CD13 $\left.{ }^{+}\right)$and MCF7 (CD13-) cells.

cKNGRE-OG and WM15 clearly demonstrated binding to HT-1080 cells (Figure 3.6B,E), whereas these agents displayed minimal binding to MCF7 cells (Figure 3.6A,D). Antibody fluorescence was membrane-associated and dispersed
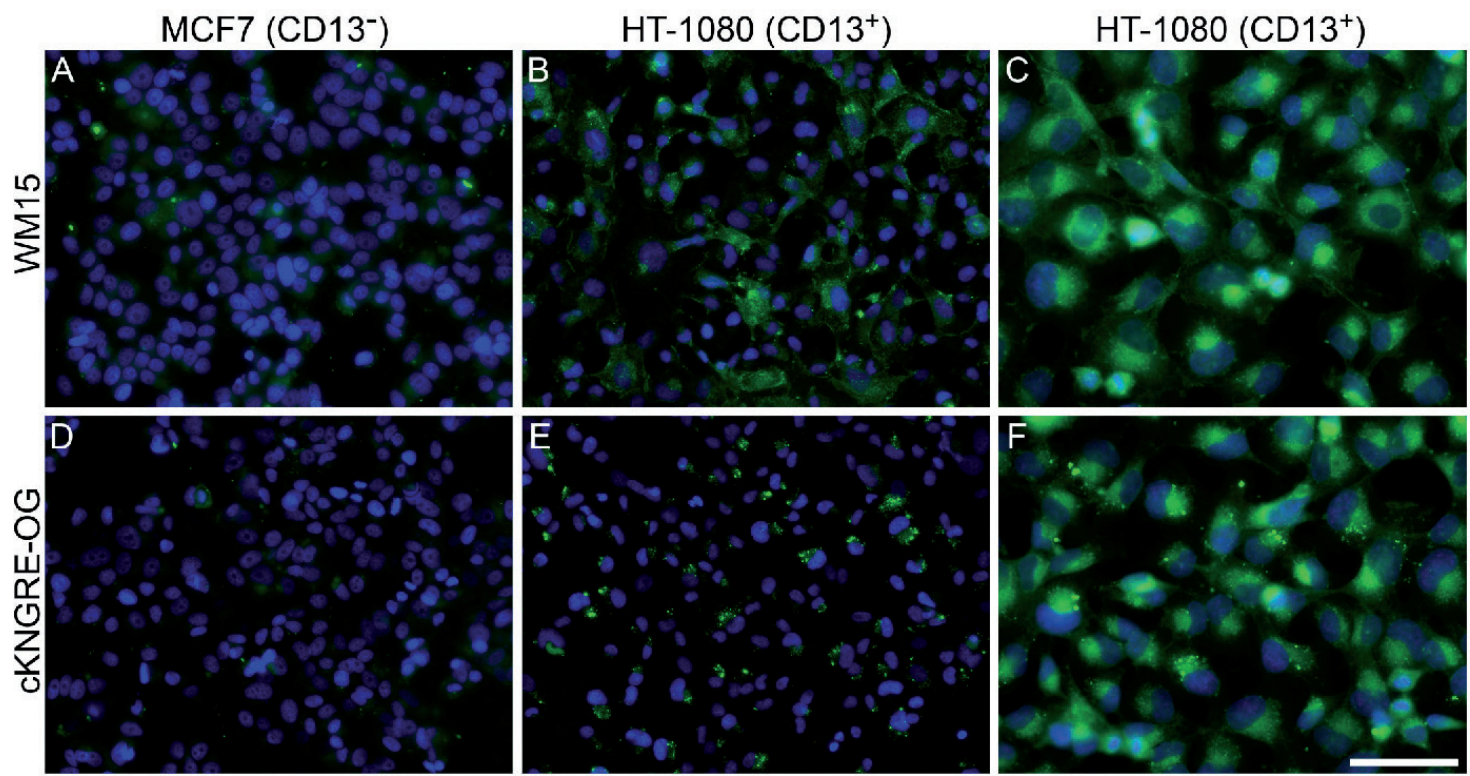

Figure 3.6 Fluorescence microscopy to evaluate in vitro binding of anti-CD13 antibody WM15 (top row: A, B, and C) and cKNGRE-OG (bottom row: D, E, and F) to CD13+ HT-1080 cells (B, C, E, and F) and CD13- MCF7 cells (A and D). Green, signal from cKNGRE-OG or WM15 antibody. Blue, signal from the nuclear staining agent DAPI. Images $\mathrm{AB}$ and DE were acquired with identical exposure times and displayed consistently to compare binding between MCF7 and HT-1080 cells. Bar equals $100 \mu \mathrm{m}$ for A, B, D, and E and $50 \mu \mathrm{m}$ for C and F. 
across the cell surface. In addition to membrane fluorescence, intense punctate fluorescence was observed in HT-1080 cells incubated with cKNGRE-OG (Figure 3.6E), suggesting internalization of the peptide. At higher magnification (Figure 3.6C, F), the localization of cKNGRE-OG and WM15 appeared similar with the exception of the bright punctate signal associated with cKNGRE-OG that was more intense than membrane-associated fluorescence. The cellular distribution to cKNGRE-OG (Figure 3.7B) with punctate fluorescence albeit at a lower fluorescent intensity than cKNGRE-OG. This distribution was similar to images of live cells.

In order to elucidate the cause of punctate fluorescence for cKNGRE-OG. HT1080 cells were incubated with cKNGRE-OG for 30 min at $4{ }^{\circ} \mathrm{C}$ or $37^{\circ} \mathrm{C}$. incubation at $4{ }^{\circ} \mathrm{C}$ reduces endocytosis and accumulation in cytoplasmic vesicles. In contrast to marked cellular internalization at $37^{\circ} \mathrm{C}$ (Figure $3.8 \mathrm{~B}$ ), at $4{ }^{\circ} \mathrm{C}$ (Figure 3.8A) there was minimal uptake of cKNGRE-OG. Furthermore, the punctate fluorescence at $37^{\circ} \mathrm{C}$ was inside the cells as determined by confocal
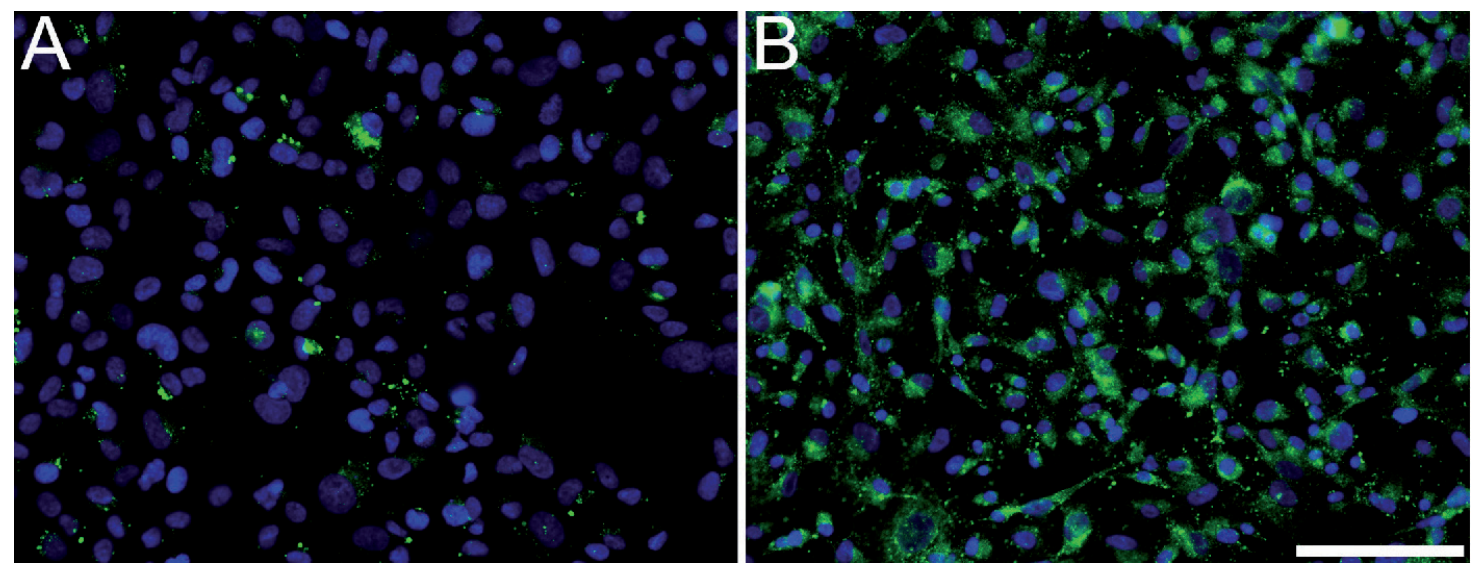

Figure 3.7 Epifluorescence microscopy to evaluate in vivo binding of linear KNGR-OG (A) and cKNGRE-OG (B) by HT-1080 cells at $37^{\circ} \mathrm{C}$. Green signals from KNGRG-OG or cKNGRE$\mathrm{OG}$ and Blue signal from the nuclear staining agent DAPI. Images were acquired with identical exposure times and displayed consistent window and level. Bar equals $100 \mathrm{~mm}$. 

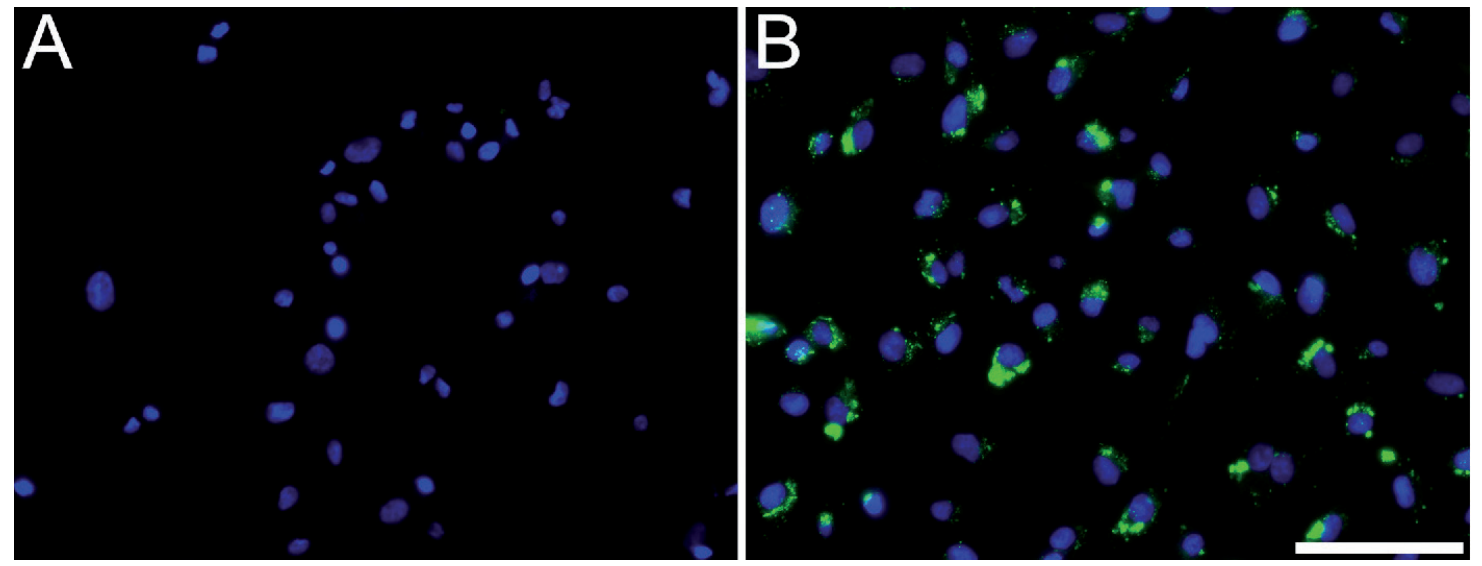

Figure 3.8 Epifluorescence microscopy to evaluate in vivo internalization of cKNGRE-OG by HT-1080 cells at $4{ }^{\circ} \mathrm{C}(\mathrm{A})$ or $37^{\circ} \mathrm{C}(\mathrm{B})$. Green signals from cKNGRE-OG and Blue signal from the nuclear staining agent DAPI. Images were acquired with identical exposure times and displayed consistent window and level. Bar equals $100 \mathrm{~mm}$.

microscopy shown in Figure 3.9. This internalized cKNGRE-OG demonstrated a pattern consistent with endosomal uptake [39, 40].

To investigate the binding affinity of the free NGR peptides and NGR-targeted LTSLs, EC $_{50}$ values were determined using flow cytometry with CD $13^{+}$HT-1080 cells. Approximately 94\% of the HT-1080 cells were positive for the WM15 antibody, indicating the presence of CD13. As shown in Figure 3.10A, cKNGREOG demonstrated 3.6-fold greater affinity for HT-1080 cells $\left(\mathrm{EC}_{50}=61.0 \mu \mathrm{M}\right)$ than did the linear KNGRG-OG $\left(\mathrm{EC}_{50}=219.9 \mu \mathrm{M}\right)$. Similarly, as shown in Figure 3.10B, LTSLs conjugated to cKNGRE $\left(\mathrm{EC}_{50}=6.2 \mu \mathrm{M}\right)$ had a 3.5-fold greater avidity than did LTSLs conjugated to linear KNGRG $\left(\mathrm{EC}_{50}=21.5 \mu \mathrm{M}\right)$. These experiments were repeated twice with similar results. These data demonstrated that the cyclic NGR had much greater affinity than the linear NGR peptide and their binding constants are sufficient for biological applications. Furthermore, the multivalent presentation of NGR peptides on the surface of liposomes increased the affinity $\sim 10$-fold over free peptides based on the concentration of NGR. 

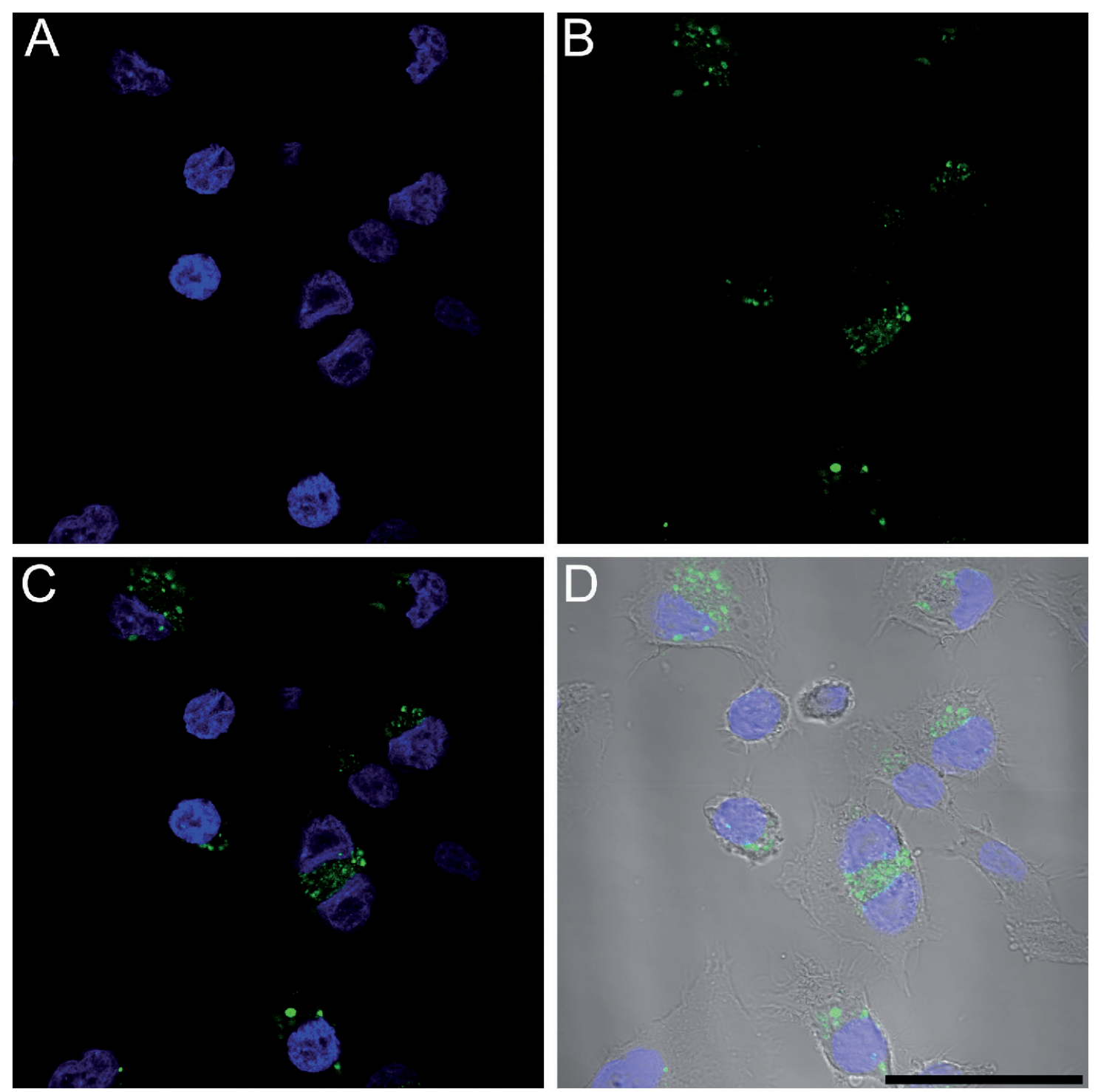

Figure 3.9 Confocal microscopy to evaluate internalization of cKNGRE-OG in HT-1080 cells at $37^{\circ} \mathrm{C}$. Blue signal from the nuclear staining agent DAPI (A), Green signals from cKNGRE-OG (B), merged image of A and B (C), and merged image of A and B with Dodt contrast to visualize cell boundaries (D). Bar equals $50 \mathrm{~mm}$.

Assuming a $100 \mathrm{~nm}$ liposome, there would be 2100 NGR ligands on the outer surface of the NGR-LTSL $[41,42]$. This corresponds to 4200 total NGR ligands per liposome. Since there are many NGRs per liposome, the affinity based on a particle basis actually increased 41,000-fold (61 $\mu \mathrm{M}$ for cKNGRE/1.5 nM for cKNGRE-targeted LTSL) due to the multivalent presentation of cNGR [43]. 

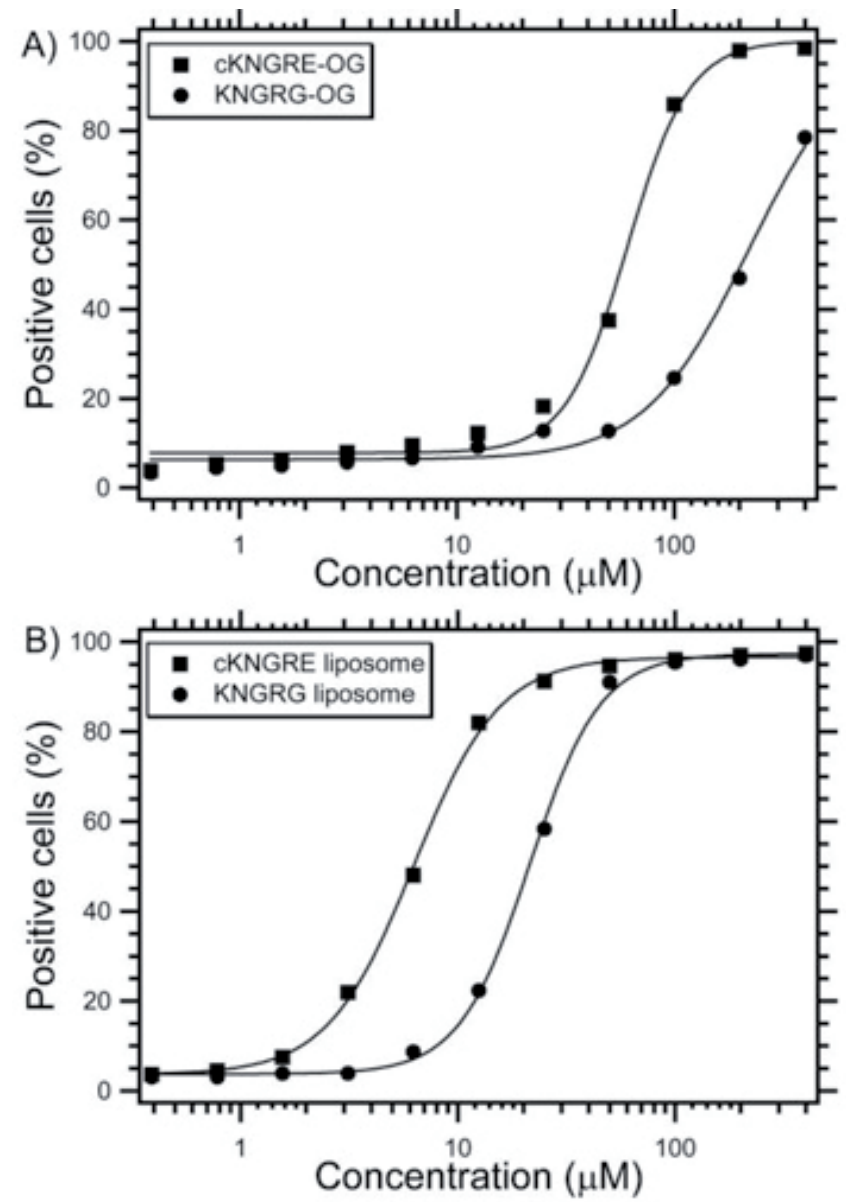

Figure 3.10 In vitro binding expressed as \% positive HT-1080 cells $\left(\mathrm{CD} 13^{+}\right)$versus concentration of cKNGRE and linear KNGRG conjugated to OG (A) and LTSL (B). The half maximal effective concentration $\left(\mathrm{EC}_{50}\right)$ was determined from the Hill equation (shown as a solid line).

\section{In vitro Dox release characteristics of cNGR-LTSLs}

The temperature dependent release of Dox from cKNGRE-targeted LTSLs was investigated with a fluorescence dequenching assay. As shown in Figure 3.11, Dox is rapidly released at $41.3{ }^{\circ} \mathrm{C}(>75 \%$ release in $<4 s)$ with minimal release at 37 ${ }^{\circ} \mathrm{C}$. These release kinetics are similar to published reports of unmodified LTSLs (slightly different formulation) [44] as well as untargeted LTSLs with an identical composition in both HEPES buffer and plasma. 


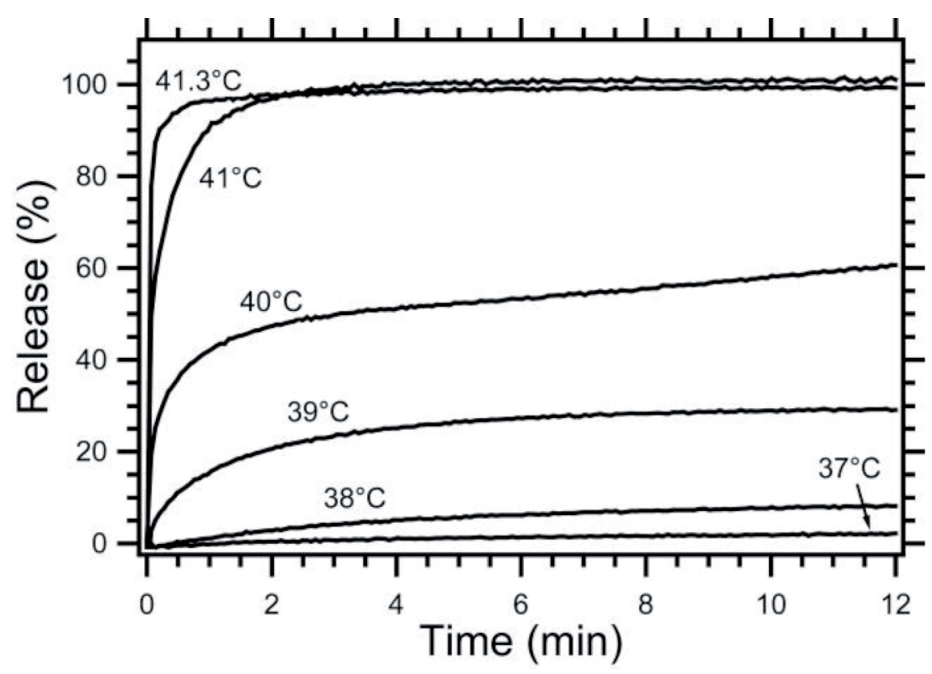

Figure 3.11 Release of Dox from cKNGRE-targeted LTSLs. Percent release is calculated by assuming $100 \%$ release with TritonÒ X-100 and $0 \%$ at $25^{\circ} \mathrm{C}$ in a HEPES buffer.

\section{Discussion}

A novel permanently cyclized NGR peptide was synthesized and evaluated for binding to $\mathrm{CD} 13^{+}$cancer cells. The synthetic strategy resulted in a sufficient yield and purity (33\% and 98\%, respectively). The permanently cyclized NGR peptide had -3.5 -fold greater affinity than the linear form both as a free peptide and on the surface of a liposome. This increase in affinity due to cyclization [22] is similar to other peptides that exhibit improved affinity when conformationally constrained [45-47]. The NGR-LTSL had greater avidity than the free NGR-OG as would be expected due to the multivalent presentation of the NGR ligand on the surface of the liposome [43]. In the cyclized peptide design, we employed the $\mathrm{C} \omega$-carboxylic acid of Glu in the ring closure process with the $\alpha$-amine of Lys so as to obtain the same number of chemical bonds in the ring as that of the originally identified and most common cyclic CNGRC peptide [2, 22], which is cyclized through a disulfide bridge. Additional modifications of the ring size and 
NGR flanking residues may further improve the binding affinity and specificity of cyclic NGR peptides.

Previous reports have demonstrated the utility of NGR peptides for both drug delivery $[23,28]$ and molecular imaging [48-50], but these works have been limited by employment of a linear or disulfide bridge form of the peptide. On the surface of a liposome or other targeting vehicle, cyclic NGR peptides formed through disulfide bridges may develop additional disulfide bridges between adjacent peptides that render the ligand ineffective [29]. The new cyclized version of NGR reported herein avoided this potential pitfall while retaining sufficient binding avidity $\left(\mathrm{EC}_{50}=6.2 \mu \mathrm{M}\right)$ to target $\mathrm{CD} 13$ in vitro. In contrast to the method of attaching linear NGR peptides to liposomes reported by Pastorino et al [28] that involved chemical attachment of the peptide to the pre-synthesized liposome, we first synthesized the PEG-lipid with the cyclic NGR targeting moiety and then synthesized the liposome. Although the previously reported method had the advantages of simplicity and an overall faster synthesis, our method offers more favorable control over liposome composition, reduced manipulations after drug loading, and reproducibility. To our knowledge, this may be the first report of a tumor vascular targeted LTSL, but tumor targeted temperature sensitive liposomes have been reported [51]. This targeted liposome may also be combined with emerging heat sources, such as high intensity focused ultrasound [52], or imaging technologies for monitoring drug delivery [53-55].

The combination of tumor vascular targeting and temperature triggered drug release from liposomes has the potential to improve therapeutic efficacy by 1) slowing the transit time of liposomes in the tumor vasculature to improve drug release, 2) improving total drug accumulation in the tumor, and 3) treating metastatic tumors not subjected to hyperthermia. The targeting of tumor 
vasculature with liposomes has the advantage over traditional tumor targeted immunoliposomes [56] of not requiring the slow process of extravasation and subsequent penetration before binding and cell uptake can occur. In contrast tumor cell antigens, tumor vascular antigens are immediately available for binding directly after intravenous administration. Furthermore, targeting angiogenic tumor vasculature is a more ubiquitous approach applicable to most solid tumors and does not require the overexpression of a tumor cell specific antigen that is often limited to a particular subtype of tumors such as HER2 [57]. Temperature triggered drug release from LTSLs has demonstrated excellent tumor control in preclinical models $[33,52]$ but this local-regional therapy is limited in its ability to treat widespread metastatic disease. The promising preclinical results of NGRtargeted non-thermally sensitive liposomes in metastatic models [28] suggests that the NGR-targeted thermally sensitive formulation reported herein may be able to provide excellent local-regional control with tumor targeted hyperthermia as well as improved therapy through NGR-targeting of unheated metastatic disease.

\section{Conclusion}

We report the synthesis of a novel cyclic NGR ligand, cKNGRE, and evaluation of its in vitro binding to $\mathrm{CD} 13^{+}$cancer cells. cKNGRE synthesis was verified with NMR and mass spectral techniques and resulted in high yield and purity. In vitro fluorescence microscopy studies revealed binding of cKNGRE-OG to CD13 $3^{+} \mathrm{HT}$ 1080 cells and minimal binding to CD13- MCF7 cells. The membrane localization of cKNGRE-OG was similar to that of the anti-CD13 WM15 antibody with the exception of a bright punctate signal associated with internalization of cKNGREOG. The cKNGRE ligand displayed 3.6-fold greater affinity for $\mathrm{CD} 13^{+}$cancer cells than did linear KNGRG. This affinity was similarly improved 10-fold for both the cyclic and linear NGR peptides when attached to the surface of LTSLs. 
The results of this study are significant because they demonstrate high avidity of an NGR-targeted LTSL without the limitation of a disulfide bridge.

\section{Acknowledgements}

This research supported in part by the Intramural Research Program of the National Institutes of Health. We thank Dr. Edyta Pawelczyk and Dr. Elaine Kay Jordan for assistance with flow cytometry, and Dr. Herman Yeh and Mr. Wesley White for ${ }^{1} \mathrm{H}-\mathrm{NMR}(600 \mathrm{MHz})$ experiments. 


\section{References}

1. Stevanovic, S., Identification of tumour-associated T-cell epitopes for vaccine development. Nat. Rev. Cancer, 2002. 2(7): p. 514-520.

2. Arap, W., R. Pasqualini, and E. Ruoslahti, Cancer treatment by targeted drug delivery to tumor vasculature in a mouse model. Science, 1998. 279(5349): p. 377-380.

3. Allen, T.M., Ligand-targeted therapeutics in anticancer therapy. Nat. Rev. Cancer, 2002. 2(10): p. 750-763.

4. Brignole, C., et al., Development of Fab' fragments of anti-GD(2) immunoliposomes entrapping doxorubicin for experimental therapy of human neuroblastoma. Cancer Lett., 2003. 197(1-2): p. 199-204.

5. Pagnan, G., et al., GD2-mediated melanoma cell targeting and cytotoxicity of liposome-entrapped fenretinide. Int. J. Cancer, 1999. 81(2): p. 268-274.

6. Gabizon, A., et al., Targeting folate receptor with folate linked to extremities of poly(ethylene glycol)-grafted liposomes: in vitro studies. Bioconjugate Chem., 1999. 10(2): p. 289-298.

7. Mantovani, L.T., et al., Folate binding-protein distribution in normal-tissues and biologicalfluids from ovarian-carcinoma patients as detected by the monoclonal-antibodies MOv18 and MOv19. Eur. J. Cancer, 1994. 30A(3): p. 363-369.

8. Campbell, I.G., et al., Folate-binding protein is a marker for ovarian-cancer. Cancer Res., 1991. 51(19): p. 5329-5338.

9. Neri, D. and R. Bicknell, Tumour vascular targeting. Nature Rev. Cancer, 2005. 5(6): p. 436-446.

10. Kumar, C.C., et al., Targeting integrins $\alpha_{2} \beta_{3}$ and $\alpha_{2} \beta_{5}$ for blocking tumor-induced angiogenesis. Adv. Exp. Med. Biol. 2000. 476: p. 169-180.

11. Curley, G.P., H. Blum, and M.J. Humphries, Integrin antagonists. Cell. Mol. Life Sci., 1999. 56(5-6): p. 427-441.

12. Hicklin, D.J. and L.M. Ellis, Role of the vascular endothelial growth factor pathway in tumor growth and angiogenesis. J. Clin. Oncol., 2005. 23(5): p. 1011-1027.

13. Ferrara, N., H.P. Gerber, and J. LeCouter, The biology of VEGF and its receptors. Nature Med., 2003. 9(6): p. 669-676.

14. Yu, J.H., C. Ustach, and H.R.C. Kim, Platelet-derived growth factor signaling and human cancer. J. Biochem. Mol. Bio., 2003. 36(1): p. 49-59.

15. Pietras, K., et al., PDGF receptors as cancer drug targets. Cancer Cell, 2003. 3(5): p. 439-443.

16. Arap, W., et al., Steps toward mapping the human vasculature by phage display. Nature Med., 2002. 8(2): p. 121-127.

17. Curnis, F., et al., Enhancement of tumor necrosis factor antitumor immunotherapeutic properties by targeted Delivery to aminopeptidase N (CD13). Nature Biotechnol., 2000. 18(11): p. 1185-1190.

18. Curnis, F., A. Sacchi, and A. Corti, Improving chemotherapeutic drug penetration in tumors by vascular targeting and barrier alteration. J. Clin. Invest., 2002. 110(4): p. 475-482.

19. Ellerby, H.M., et al., Anti-cancer activity of targeted pro-apoptotic peptides. Nature Med., 1999. 5(9): p. 1032-1038.

20. Hood, J.D., et al., Tumor regression by targeted gene delivery to the neo-vasculature. Science, 2002. 296(5577): p. 2404-2407.

21. Pasqualini, R., et al., Aminopeptidase- $N$ is a receptor for tumor-homing peptides and a target for inhibiting angiogenesis. Cancer Res., 2000. 60(3): p. 722-727. 
22. Colombo, G., et al., Structure-activity relationships of linear and cyclic peptides containing the NGR tumor-homing motif. J. Bio. Chem., 2002. 277(49): p. 47891-47897.

23. Garde, S.V., et al., Binding and internalization of NGR-peptide-targeted liposomal doxorubicin (TVT-Dox) in CD13-expressing cells and its antitumor effects. Anti-Cancer Drugs, 2007. 18(10): p. 1189-1200.

24. Pastorino, F., et al., Vascular damage and anti-angiogenic effects of tumor vessel-targeted liposomal chemotherapy. Cancer Res., 2003. 63(21): p. 7400-7409.

25. Fidler, I.J. and L.M. Ellis, The implications of angiogenesis for the biology and therapy of cancer metastasis. Cell, 1994. 79(2): p. 185-188.

26. Majhen, D., et al., Disulfide bond formation in NGR fiber-modified adenovirus is essential for retargeting to aminopeptidase- $N$. Biochem. Biophys. Res. Commun., 2006. 348(1): p. 278-287.

27. Moffatt, S., et al., Successful in vivo tumor targeting of prostate-specific membrane antigen with a highly efficient J591/PEI/DNA molecular conjugate. Gene Ther., 2006. 13(9): p. 761-772.

28. Pastorino, F., et al., Targeting liposomal chemotherapy via both tumor cell-specific and tumor vasculature-specific ligands potentiates therapeutic efficacy. Cancer Res., 2006. 66(20): p. 10073-10082.

29. Corti, A. and M. Ponzoni, Tumor vascular targeting with tumor necrosis factora and chemotherapeutic drugs. Ann. N. Y. Acad. Sci., 2004. 1028: p. 104-112.

30. Chowdhry, B.Z., et al., Multicomponent phase-transitions of diacylphosphatidylethanolamine dispersions. Biophys. J., 1984. 45(5): p. 901-904.

31. Kong, G. and M.W. Dewhirst, Hyperthermia and liposomes. Int. J. Hyperth., 1999. 15(5): p. 345-370.

32. Mills, J.K. and D. Needham, Lysolipid incorporation in dipalmitoylphosphatidylcholine bilayer membranes enhances the ion permeability and drug release rates at the membrane phase transition. Biochim. Biophys. Acta-Biomembr., 2005. 1716(2): p. 77-96.

33. Kong, G., R.D. Braun, and M.W. Dewhirst, Hyperthermia enables tumor-specific nanoparticle delivery: effect of particle size. Cancer Res., 2000. 60(16): p. 4440-4445.

34. Ferrara, K., Driving delivery vehicles with ultrasound. Adv. Drug Deliv. Rev., 2008. 60(10): p. 1097-1102.

35. Mayer, L.D., et al., Influence of vesicle size, lipid-composition, and drug-to-lipid ratio on the biological-activity of liposomal doxorubicin in mice. Cancer Res., 1989. 49(21): p. 5922-5930.

36. Fenske, D.B., N. Maurer, and P. Cullis, Liposomes. 2nd ed. 2003: Oxford University Press. 424.

37. Kates, S.A., et al., A novel, convenient, 3-dimensional orthogonal strategy for solid-phase synthesis of cyclic-peptides. Tetrahedron Lett., 1993. 34(10): p. 1549-1552.

38. Kates, S.A., et al., Solid-Phase $N$-glycopeptide synthesis using allyl side-chain protected fmocamino acids. Tetrahedron Lett., 1994. 35(7): p. 1033-1034.

39. Wang, X., et al., NGR-modified micelles enhance their interaction with CD13-overexpressing tumor and endothelial cells. J. Control. Release, 2009. 139(1): p. 56-62.

40. Hansen, G.H., et al., The coronavirus transmissible gastroenteritis virus causes infection after receptor-mediated endocytosis and acid-dependent fusion with an intracellular compartment. J. Viro., 1998. 72(1): p. 527-534.

41. Kirpotin, D., et al., Sterically stabilized nti-HER2 immunoliposomes: design and targeting to human breast cancer cells in vitro. Biochem., 1997. 36(1): p. 66-75.

42. Huang, C. and J.T. Mason, Geometric packing constraints in egg-phosphatidylcholine vesicles. Proc. Natl. Acad. Sci. U. S. A., 1978. 75(1): p. 308-310. 
43. Mammen, M., S.K. Choi, and G.M. Whitesides, polyvalent interactions in biological systems: implications for design and use of multivalent ligands and inhibitors. Angew. Chem. Int. Ed., 1998. 37(20): p. 2755-2794.

44. Mills, J.K. and D. Needham, Lysolipid incorporation in dipalmitoylphosphatidylcholine bilayer membranes enhances the ion permeability and drug release rates at the membrane phase transition. Biochim. Biophys. Acta-Biomembr., 2005. 1716(2): p. 77-96.

45. Lee, G., et al., Strong inhibition of fibrinogen binding to platelet receptor $-a_{I I b} b_{3}$ by $R G D$ sequences installed into a presentation scaffold. Protein Eng., 1993. 6(7): p. 745-754.

46. Mousa, S.A., et al., Platelet GPIIb/IIIa binding characteristics of small molecule RGD mimetic: distinct binding profile for roxifiban. Br. J. Pharmacol., 2001. 133(3): p. 331-336.

47. Huang, G.F., et al., Affinity manipulation of surface-conjugated RGD peptide to modulate binding of liposomes to activated platelets. Biomaterials, 2008. 29(11): p. 1676-1685.

48. von Wallbrunn, A., et al., In vivo optical imaging of CD13/APN-expression in tumor xenografts. J. Biomed. Opt., 2008. 13(1): p. - .

49. Dirksen, A., et al., Design and synthesis of a bimodal target-specific contrast agent for angiogenesis. Org. Lett., 2004. 6(26): p. 4857-4860.

50. Buehler, A., et al., cNGR: A novel homing sequence for CD13/APN targeted molecular imaging of murine cardiac angiogenesis in vivo. Arterioscler. Thromb. Vasc. Bio., 2006. 26(12): p. 2681-2687.

51. Puri, A., et al., HER2-specific affibody-conjugated thermosensitive liposomes (Affisomes) for improved delivery of anticancer agents. J. Liposome Res., 2008. 18(4): p. 293-307.

52. Dromi, S., et al., Pulsed-high intensity focused ultrasound and low temperature sensitive liposomes for enhanced targeted drug delivery and antitumor effect. Clin. Cancer Res., 2007. 13(9): p. 2722-2727.

53. Ponce, A., et al., Magnetic resonance imaging of temperature-sensitive liposome release: drug dose painting and antitumor effects. J. Natl. cancer Inst., 2007. 99(1): p. 53-63.

54. Viglianti, B., et al., In vivo monitoring of tissue pharmacokinetics of liposomeldrug using MRI: illustration of targeted delivery. Magn. Reson. med., 2004. 51(6): p. 1153-1162.

55. Langereis, S., et al., A temperature-sensitive liposomal ${ }^{1} \mathrm{H} C E S T$ and ${ }^{19} \mathrm{~F}$ contrast agent for $M R$ image-guided drug delivery. J. Am. Chem. Soc., 2009. 131(4): p. 1380-1381.

56. Torchilin, V.P., Recent Advances with Liposomes as Pharmaceutical Carriers. Nature Rev. Drug Discov., 2005. 4(2): p. 145-160.

57. Park, J.W., et al., Tumor targeting using anti-HER2 immunoliposomes. J. Control. Release, 2001. 74(1-3): p. 95-113. 


\section{Chapter 4}

\section{Synthesis and characterization of image-able polyvinyl alcohol microspheres for image-guided chemoembolization}

Ayele H. Negussie ${ }^{1,3}$, Matthew R. Dreher ${ }^{1}$, Carmen Gacchina Johnson ${ }^{1}$,

Yiqing Tang ${ }^{2}$, Andrew L. Lewis ${ }^{2}$, Gert Storm ${ }^{3,4}$, Karun V. Sharma ${ }^{1}$, Bradford J. Wood $^{1}$

${ }^{1}$ Radiology and Imaging Sciences, Clinical Center, National Institutes of Health, Bethesda, MD, 20892, United States

${ }^{2}$ Biocompatibles UK Ltd., Farnham Business Park, Weydon Lane, Farnham, Survey GU98QL, UK

${ }^{3}$ Department of Targeted Therapeutics, MIRA Institute, University of Twente, Enschede, The Netherlands

${ }^{4}$ Department of Pharmaceutics, Utrecht Institute for Pharmaceutical Sciences, Utrecht University, Utrecht, The Netherlands

Published in: J. Mater. Science: Mater. Med., (26): 2015 


\section{Abstract}

Therapeutic embolization of blood vessels is a minimally invasive, catheter-based procedure performed with solid or liquid emboli to treat bleeding, vascular malformations, and vascular tumors. Hepatocellular carcinoma (HCC) affects about half a million people per year. When unresectable, HCC is treated with embolization and local drug therapy by transarterial chemoembolization (TACE). For TACE, drug eluting beads (DC Bead $\left.{ }^{\oplus}\right)$ may be used to occlude or reduce arterial blood supply and deliver chemotherapeutics locally to the tumor. Although this treatment has been shown to be safe and to improve patient survival, the procedure lacks imaging feedback regarding the location of embolic agent and drug coverage. To address this shortcoming, herein we report the synthesis and characterization of image-able drug eluting beads (iBeads) from the commercial DC Bead product. Two different radiopaque beads were synthesized. In one approach, embolic beads were conjugated with 2,3,5-triiodobenzyl alcohol in the presence of 1,10-carbonyldiimidazol to give iBead I. iBead II was synthesized with a similar approach but instead using a trimethylenediamine spacer and 2,3,5-triiodobenzoic acid. Doxorubicin was loaded into the iBeads II using a previously reported method. Size and shape of iBeads were evaluated using an upright microscope and their conspicuity assessed using a clinical CT and micro-CT. Bland and Doxloaded iBeads II visualized with both clinical CT and microCT. Under microCT, individual bland and Dox loaded beads had a mean attenuation of $7904 \pm 804$ and 11,873.96 $\pm 706.12 \mathrm{HU}$, respectively. These iBeads have the potential to enhance image guided TACE procedures by providing localization of embolic-particle and drug. 


\section{Introduction}

Embolization is a minimally invasive, catheter-based procedure used to mechanically occlude blood vessels feeding a tumor or other target tissue to obtain therapeutic benefit $[1,2]$. There are numerous pathologies that can be treated with embolization therapy, including bleeding [3-6], vascular malformations [7-12], and renal and hepatic tumors (both benign and malignant) [13-15]. Embolizing agents rely on mechanical obstruction to occlude vessels and the choice of a given therapy depends on the material properties and the desired clinical outcomes.

The first embolic agent in clinical use was autologous blood clots which were readily available and biocompatible [16], however, quick lysis of the clot limited the durability of the occlusion and utility. There are currently numerous types of embolic agents available including coils (push-able, injectable or detachable), solid synthetic polymers in the form of particles and microspheres (e.g., PVA hydrogels, trisacryl gelatin (TGA), poly(d-l-lactide), polymethylmethacrylatebased (PMMA) hydrogels), natural polymers (e.g., gelatin sponge, wax, starch, alginate and albumin microspheres) and liquids (e.g., cyanoacrylate glue, alcohol, ethylene vinyl acetate copolymer solutions) [12, 16-24]. PVA, TGA, and PMMA microspheres are symmetrical, smooth and available in precisely calibrated size ranges [12, 25-29]. PVA microspheres with sulfonate groups (DC Bead ${ }^{\oplus}$ ) or sodium acrylate groups (HepaSphere ${ }^{\oplus}$ and Tandem ${ }^{\oplus}$ ), respectively, are commonly used as drug carriers for chemoembolization to treat patients with hepatocellular carcinoma (HCC) [30-32] and generically known as drug-eluting beads (DEBs).

HCC affects approximately 500,000 people worldwide, every year [33]. It is the second most common cause of cancer mortality [34]. The curative options that are currently available in clinics depend upon the stage of the disease. For example, 
surgical resections, liver transplant, radiofrequency ablation (RFA), and vascular occlusion followed by RFA are options for early stage tumors and yield improved survival $[33,35-42]$. When these options fail, transarterial embolization (TAE), transarterial chemoembolization (TACE; i.e., infusion of a chemotherapeutic followed by embolization), or the use of drug-eluting bead (DEB) transarterial chemoembolization (DEB-TACE) [35, 43-46] are typical choices for palliative treatment [33]. Since the early 1970s, TAE and later TACE have become common clinical practice $[1,33,37,44,47-63]$ and have improved the survival of patients with unresectable HCC [33, 44, 64, 65]. For instance, Yamada, et al. reported TAE with gelatin foam treatment for 120 unresectable hepatoma patients resulted in a cumulative one-year survival rate of $44 \%$ compared to $7 \%$ with conventional chemotherapy alone [66]. Similarly, Konno et al., in the early 1980s, introduced anticancer drug (SMANCS) in combination with Ethiodol ${ }^{\circledR}$ as image-guided TACE, which resulted in 10-99\% tumor size reduction in $90 \%$ of patients with unresectable HCC [67]. Recently, uniformly sized microspheres with high drug loading capacity have emerged in the clinic as DEBs. These microspheres can be used in bland embolotherapy or more commonly loaded with chemotherapeutics to create a drug delivery device $[31,32,60,68-72]$. For example, DEBs can simultaneously occlude arterial blood supply and deliver chemotherapeutics locally to the tumor (DEB-TACE) $[57,59,62,70,71,73,74]$ providing theoretical benefit in limiting recurrence or time to tumor progression. One major drawback, however, with the current therapies is the lack of imaging feedback regarding the embolic bead location and drug coverage. Such information could inform and optimize therapy. To address this shortcoming, herein we report the synthesis and characterization of image-able drug eluting beads (iBeads) for real-time localization of embolic particles and drug during DEB-TACE. 
The embolic particles used in this work are based upon sulfonate-modified PVA microspheres $\left(\mathrm{DC} \mathrm{Bead}^{\oplus}\right)$ which have been used in clinics as embolization devices for the treatment of hypervascular lesions and vascular malformations [59]. Chemically, these PVA microspheres contain abundant chemically modifiable and reactive alcohol groups. Some of the free alcohol groups of the PVA chains have been modified to attach sulfonic acid bearing moieties and are used to sequester positively charged drugs (e.g., doxorubicin, irinotecan, topotecan) [59, 75-77]. In this study, a portion of the hydroxyl groups of the PVA chain was modified with a radio-dense species to make the microsphere detectable by $\mathrm{X}$-ray.

\section{Materials and Methods}

\section{Materials}

DC Bead ${ }^{\oplus}$ intermediate (Acrylamido Polyvinyl Alcohol-co-Acrylamido-2methylpropane sulfonate microspheres, dried to a free-flowing powder and untainted) was obtained from Biocompatibles UK Ltd, a BTG International group company, Camberley, UK. Anhydrous dimethyl sulfoxide (DMSO), 1,1'-Carbonyldiimidazol (CDI), 2,3,5-Triiodobenzyl alcohol, 2,3,5-Triiodobenzoic acid, N,N'-Diisopropylcarbodiimide (DIC), 1-Hydroxybenzotriazole hydrate (HOBt), 4-(Dimethylamino)pyridine (DMAP), Trimethylenediamine, Triethylamine $(\mathrm{Et} 3 \mathrm{~N})$, and anhydrous Dichloromethane (DCM) were purchased from Sigma Aldrich (Milwaukee, WI). Doxorubicin (Dox) was obtained from Bedford Laboratories ${ }^{\circledR}$ (Bedford, OH). De-ionized water (DI water) was obtained from in house filtration system (Hydro Inc. Rockville, MD). 


\section{General methods}

\section{Evaluation of iBeads with microscopy}

The size and appearance of beads during various steps of synthesis and Dox loading were examined and imaged in a chamber slide (Electron Microscopy Sciences; $\sim 150 \mathrm{~mL}$ bead and DI water suspension). Bright field images were acquired with a $5 x$ objective on an upright microscope (Zeiss Axioimager M1, Thornwood, NY) equipped with a color CCD camera (AxioVision, Zeiss).

\section{Phantom preparation}

In order to assess the radiopacity, beads were suspended in an agarose matrix at bead concentrations (sediment bead volume percent) that is relevant for in vivo applications. Bead containing agarose phantoms $(0.5 \% \mathrm{w} / \mathrm{v})$ with various concentrations (sedimented bead volume percent ranging from 0, 3.1, 6.2 and $12.5 \%$ ) were prepared by adding a $1 \%$ agarose (w/v, in DI water) solution (at 55 $\left.{ }^{\circ} \mathrm{C}\right)$ to an equal volume of bead suspension in DI water. The solutions were mixed while allowing the agarose to slowly gel (over ice), resulting in a homogeneous distribution of beads. [Note: The bead volume percent is sedimented bead volume due to gravity alone and does not account for aqueous solution between the packed beads or altered bead packing efficiency].

\section{In vitro evaluation of radiopacity of iBeads with clinical and Micro CT}

\section{Clinical CT}

Clinical CT imaging and analysis of iBead containing phantoms was performed on a 256 Slice CT (Philips, Andover, MA) with the following settings to determine the attenuation: $465 \mathrm{mAs}$ tube current, $80 \mathrm{keV}$ tube voltage, $1 \mathrm{~mm}$ slice thickness, 
$0.5 \mathrm{~mm}$ overlap. The average attenuation of an $80 \mathrm{~mm} 2$ rectangular region in the middle slice of a given phantom was measured using OsiriX Software (V.5.02 64-bit).

\section{MicroCT}

MicroCT imaging and analysis of iBead containing phantoms was performed with a SkyScan 1172 high-resolution micro-CT (SkyScan, Konitch, BE) to evaluate the radiopacity of each individual bead, as well as intra-bead distribution of iodine. The radiopaque microspheres were imaged at $5 \mu \mathrm{m}$ resolution, $78 \mathrm{kV}, 127$ microAmps, using a $0.5 \mathrm{~mm}$ Aluminum filter. The average attenuation of individual beads was measured and reported as the mean and standard error $(n=10)$.

\section{Doxorubicin loading into iBeads and elution}

iBeads were loaded with Doxorubicin (Dox) according to previously reported method $[58,59]$. Briefly, $250 \mu$ of thoroughly washed iBeads was immersed into $0.5 \mathrm{ml}$ of Dox $(20 \mathrm{mg} / \mathrm{ml}$ DI water) solution and shaken for 3 hours at room temperature. The Dox-loaded iBeads were used for subsequent experiments. Dox elution kinetics from iodinated beads $(100-300 \mu \mathrm{m}$ and $300-500 \mu \mathrm{m})$ were measured with a previously reported technique [59].

\section{Experimental procedures}

\section{Method I: Conjugation of 2,3,5-Triiodobenzyl alcohol on to DC Beads (iBead I)}

After washing the beads (1) $(200 \mathrm{mg})$ with DMSO (3X $5 \mathrm{~mL})$, the beads were allowed to swell in DMSO $(20 \mathrm{~mL})$ for 30 minutes at $50{ }^{\circ} \mathrm{C}$. A portion of beads hydroxyl group were activated by stirring the suspended beads with CDI (800 mg) (CDI: $\mathrm{OH}=\sim 1.2: 1)$ in the presence of catalytic amount of triethylamine 
(0.12 equivalent) at $50{ }^{\circ} \mathrm{C}$ for 24 hours. The reaction mixture was cooled to room temperature and washed quickly with a cocktail of DMSO and DCM (1:1) and finally with DMSO alone. The washed beads were immediately transferred into a reaction flask containing a solution of 2,3,5-triiodobenzyl alcohol $(971.7 \mathrm{mg})$ in DMSO $(10 \mathrm{~mL})$ and stirred for 24 hours at $50{ }^{\circ} \mathrm{C}$ (Figure 4.1). The resulting product cooled to room temperature and was washed thoroughly with DMSO: DCM (1:1), followed by DMSO alone. Finally, the DMSO was exchanged with DI water (under continuous agitation) and the image-able beads thoroughly washed with saline and DI water consecutively. The clean beads (iBead I) were suspended in DI water until further analysis.

\section{Method II: Conjugation of 2,3,5-triiodobenzoic acid on to DC Beads through trimethylenediamine spacer.}

Methods II a: Grafting a spacer on to the DC Beads (4)

The beads were activated as described in method I above $(200 \mathrm{mg}$ of beads and $1.3 \mathrm{~g}$ of CDI) and reacted with excess 1,3 -diaminopropane at $50{ }^{\circ} \mathrm{C}$ for 24 hours (Figure 4.2). The reaction mixture was cooled and washed thoroughly as mentioned in method I to give the intermediate (4). Conjugation of the trimethylenediamine was confirmed by the Kaiser test [78].

\section{Methods II b: Conjugation of 2,3,5-triiodobenzoic acid with trimethylenediamine grafted beads (iBead II)}

A solution of 2,3,5-triiodobenzoic acid (2.4 g) in DMSO $(20 \mathrm{ml})$ was activated with DIC (604.5 mg), HOBt (634.2 mg) and DMAP (586.4 mg) for 30 minutes at room temperature and the trimethylenediamine grafted beads (4) were added (Figure 4.2). The resulting reaction mixture was stirred at $40{ }^{\circ} \mathrm{C}$ for $24 \mathrm{hrs}$. After cooling, the beads (iBead II) were thoroughly washed as described in method I. Alternatively, iBead II was synthesized first by activating the 2,3,5-triiodobenzoic 


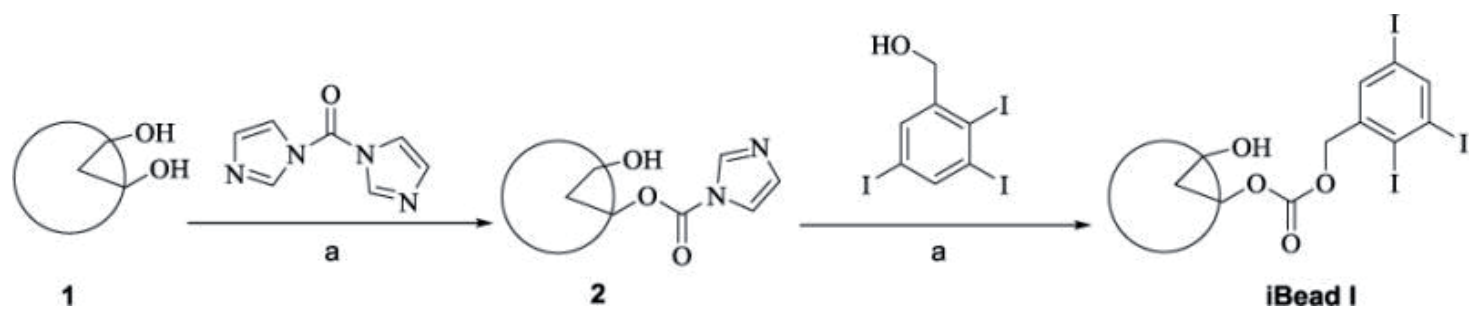

Figure 4.1 Mild activation of beads (1) with CDI and conjugation with 2,3,5-triiodobenzyl alcohol. A) DMSO and DIEA, $\mathrm{t}=50{ }^{\circ} \mathrm{C}$

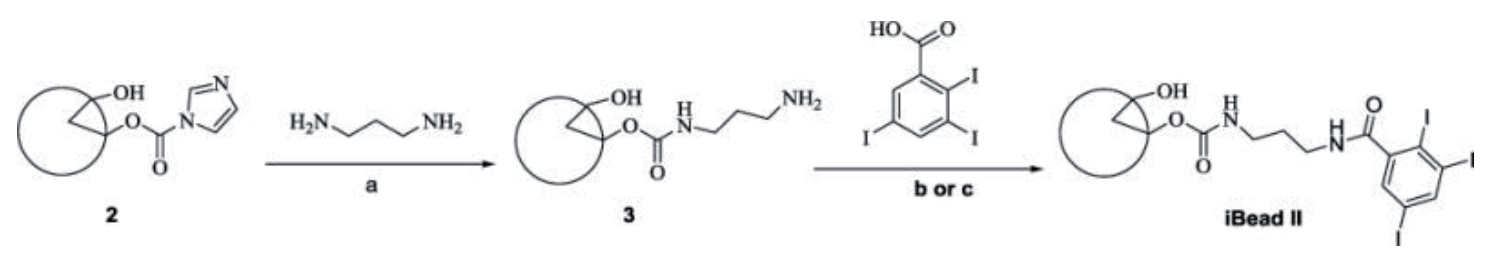

Figure 4.2 Grafting the trimethylenediamine onto CDI activated beads (2) and conjugation of 2,3,5-triiodobenzoic acid, a) DMSO and DIEA, $\mathrm{t}=50^{\circ} \mathrm{C}$. b) DMSO, DIC, HOBt and DMAP or c) DMSO and CDI.

acid (TIBA) with CDI as follows: $40 \mathrm{~g}$ of 2,3,5-triiodobenzoic acid (TIBA) was dissolved in $100 \mathrm{ml}$ of anhydrous DMSO. The compound was activated by adding $13 \mathrm{~g}$ of CDI powder under magnetic stirring. The solution gradually became turbid and viscous after about 30 to $60 \mathrm{~min}$. The mixture was then added into the trimethylenediamine grafted bead suspension in $100 \mathrm{ml}$ of DMSO. The suspension was stirred at $50{ }^{\circ} \mathrm{C}$, over 24 hours and was protected from light. The beads were washed with diethyl ether and DMSO mixture, then by deionized water.

\section{Results}

\section{Synthesis and radiopacity}

\section{Method I}

The beads were successfully activated with CDI under very mild conditions and conjugated with 2,3,5-triiodobenzyl alcohol (Figure 4.1). Microscopic image 
comparison of raw and 2,3,5-triiodobenzyl alcohol conjugated beads (iBeads) revealed that the bead size was slightly reduced (Figure 4.3).

The 2,3,5-triiodobenzyl alcohol conjugated bead radiopacity was assessed both in clinical and microCT (Figure 4.4). With clinical CT, visualization is based on radiopacity of the beads in an agarose phantom per a given bead volume (Figure 4.4A). A 3.1\% packed bead volume showed a mean attenuation of $26 \pm 15 \mathrm{HU}$ and increased to $41 \pm 16 \mathrm{HU}$ and $74 \pm 25 \mathrm{HU}$ as the packed bead volume increased to 6.2 and $12.5 \%$, respectively. With microCT, individual beads had a mean attenuation of $619.96 \pm 605.08 \mathrm{HU}(\mathrm{n}=10)$, Figure 4.4B.

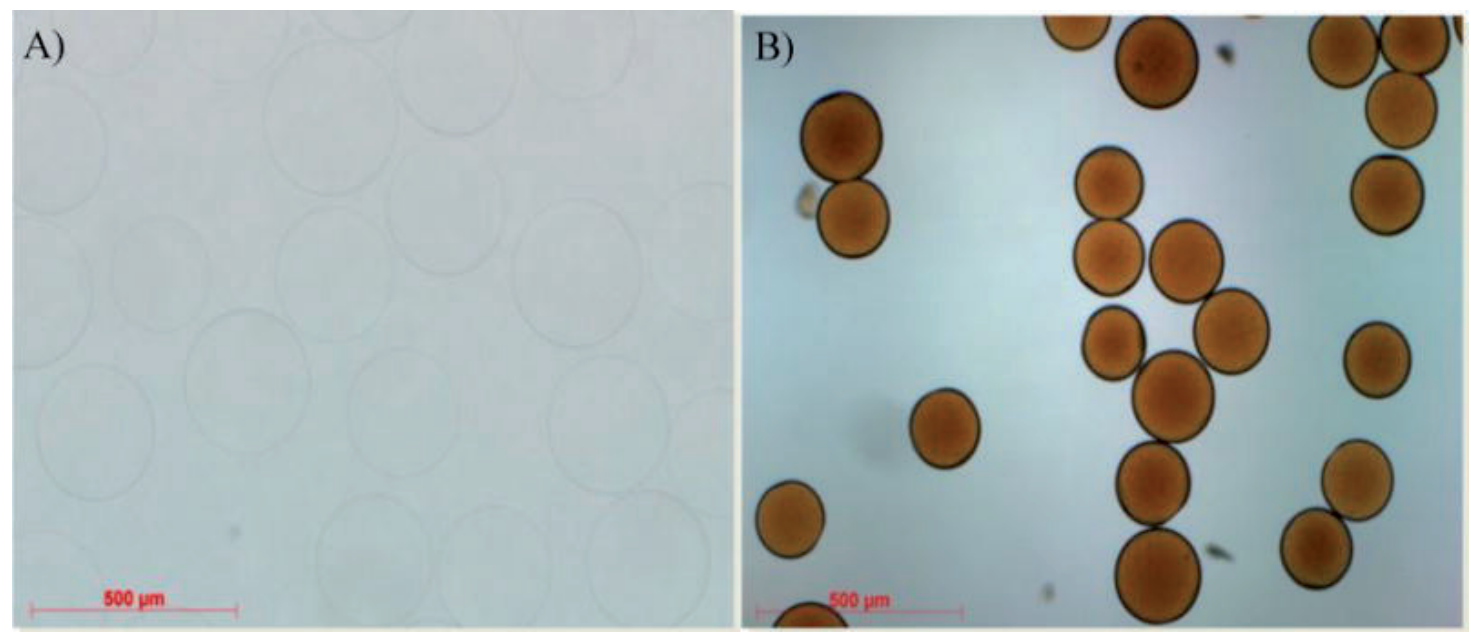

Figure 4.3 Size and appearance comparison of (A) raw bead and (B) iBead II under light microscopy. Note: conjugation of 2,3,5-triiodobenzyl acid reduces the size and changes the appearance of the beads.
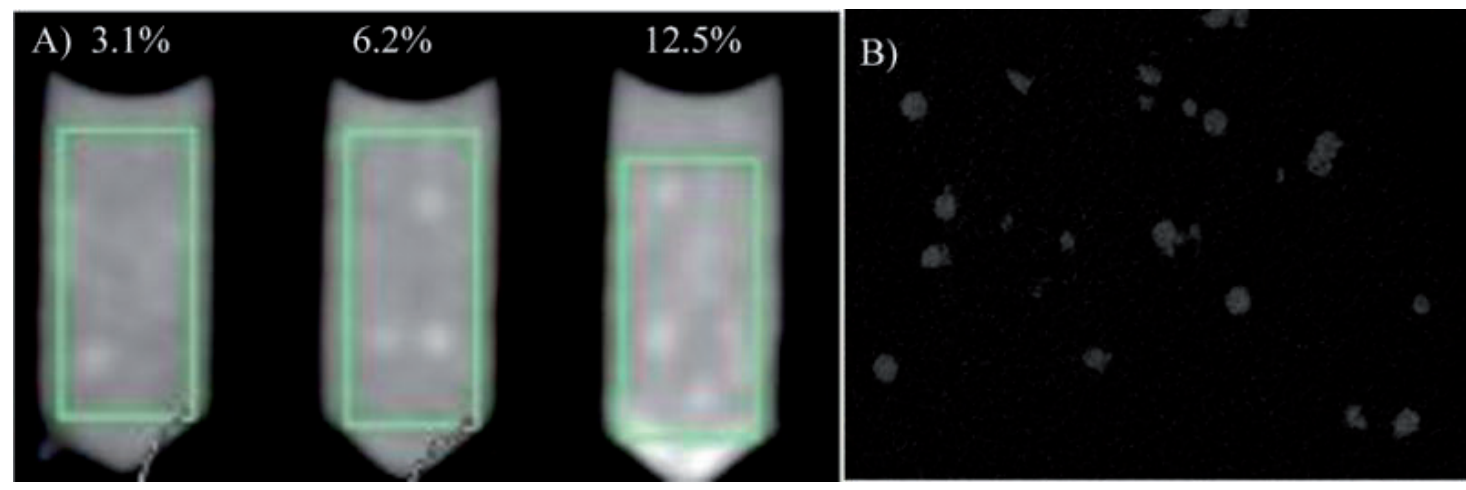

Figure 4.4. Clinical CT (A) and Micro CT images (B) of iBead I. 


\section{Method II a}

Trimethylenediamine was successfully grafted onto PVA beads as confirmed with Kaiser Test.

\section{Method II $b$}

The 2,3,5-triiodobenzoic acid was conjugated on the beads (iBead II) as confirmed by the disappearance of the bluish color upon performing the Kaiser Test. Alternatively, the reaction progress was monitored with HPLC by measuring the residual diamine in the reaction mixture after converting into $\mathrm{N}$-(3-aminopropyl)2-phenylacetamide with benzoyl chloride. Trimethylenediamine concentration gradually decreased over a period of 15 hours.

\section{Clinical and microCT}

The radiopacity was assessed with both clinical and microCT (Figure 4.6). With the clinical CT, visualization is based on radiopacity of the beads per a given bead volume. A 3.1\% packed bead volume showed a mean radiopacity of $129 \pm 33 \mathrm{HU}$ and increased to $269 \pm 53 \mathrm{HU}$ and $444 \pm 83 \mathrm{HU}$ as the (iBead II) packed bead
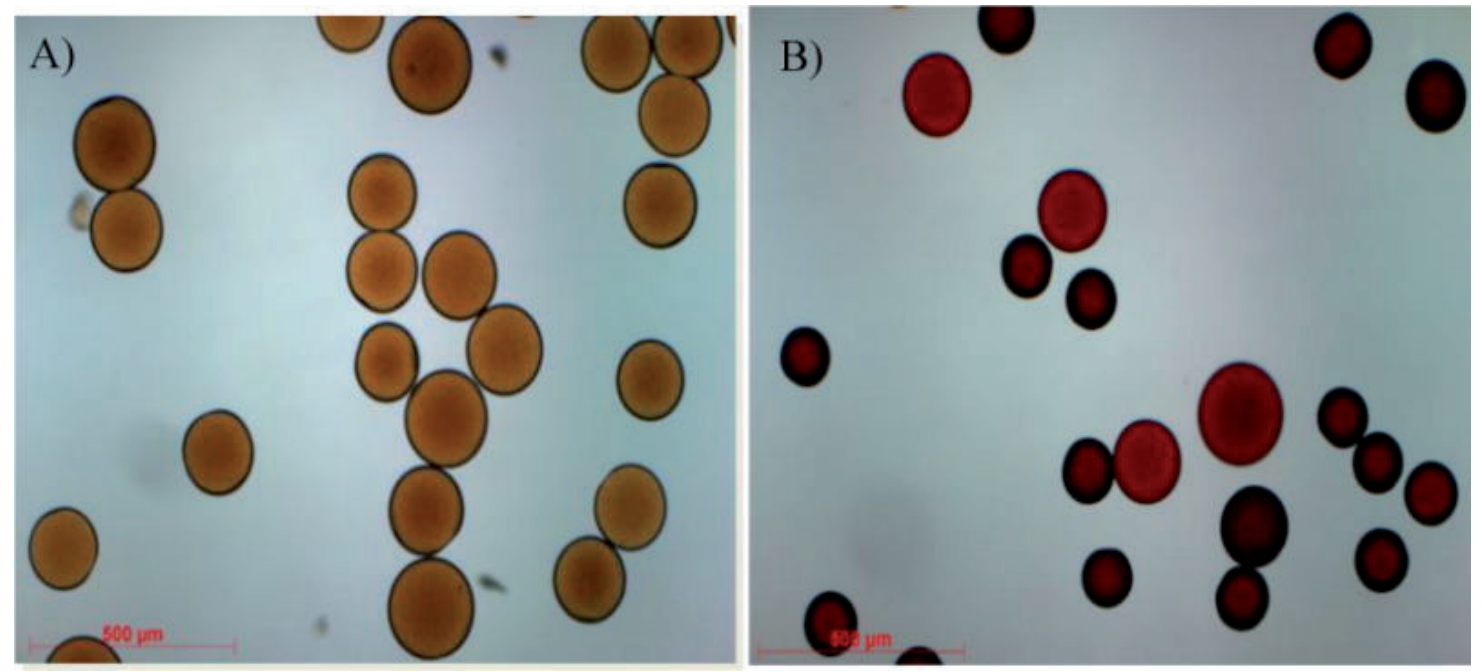

Figure 4.5 Size comparison of raw (A) and Dox loaded (B) iBead II under Light microscopy. Note: Dox loading reduces the size of the beads. 


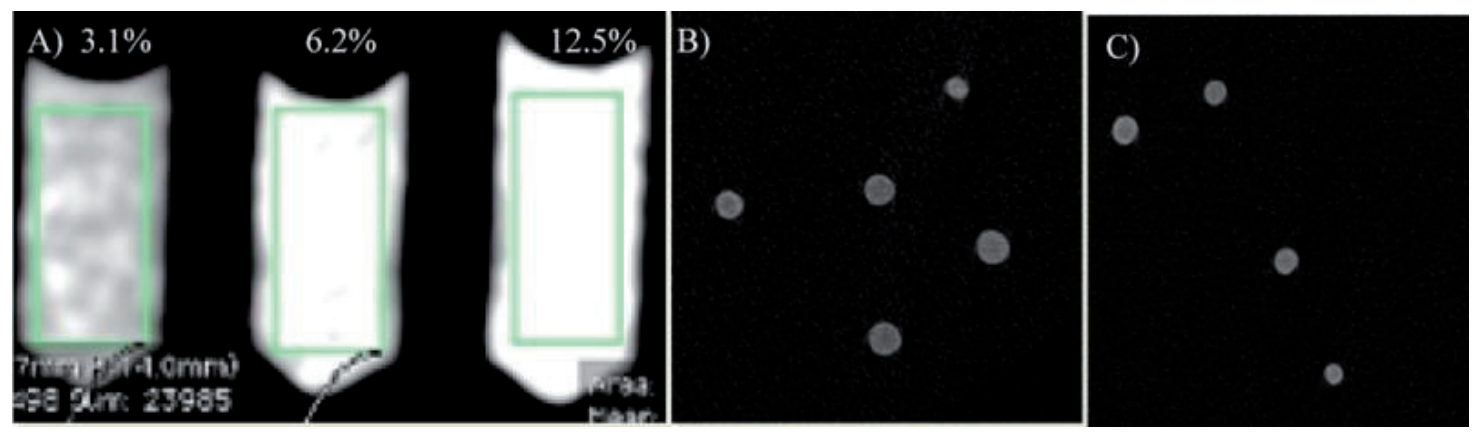

Figure 4.6 A) Clinical CT image of bland iBeads II (the green rectangle shows the measured area, $0.8 \mathrm{~cm} 2$ ), B) microCT images of bland and (C) Dox loaded iBeads II. For microCT, 3.1\%, 6.2\% and $12.5 \%(\mathrm{v} / \mathrm{v})$ iBeads II pack phantom was used.

volume increased to 6.2 and $12.5 \%$, respectively (Figure 4.6A). Using microCT, individual bland and Dox loaded beads showed a mean attenuation of $7903 \pm 804$ HU and $11873.96 \pm 706.12$, respectively $(n=10)$, see figure 4.6. Different bead size showed different iodine content and attenuation under microCT (see table below and figure 4.7).

Microscopic comparison of raw beads (Figure 4.3A) and beads yielded from Method II b (Figure 4.5A) and thereafter Dox-loaded (Figure 4.5B) suggests that the conjugated hydrophobic moiety (phenyl group) results in a decreased bead size.

Table Solid content, iodine content and $\mu \mathrm{CT}$ attenuation of iBeads II with different sizes

\begin{tabular}{llll}
\hline Bead size $(\mu \mathrm{m})$ & $\begin{array}{l}\text { Solid content of hydrated } \\
\text { beads } \\
(\%)\end{array}$ & $\begin{array}{l}\text { Iodine content of } \\
\text { dry beads } \\
(\%)\end{array}$ & $\begin{array}{l}\text { MicroCT Attenuation } \\
(\mathrm{HU})\end{array}$ \\
\hline $70-150$ & 27.2 & 41.9 & $9758 \pm 1476$ \\
$100-300$ & 24.8 & 44.3 & $8037 \pm 1142$ \\
$300-500$ & 23.7 & 45.1 & $8243 \pm 1240$ \\
$500-700$ & 23.0 & 45.6 & $7326 \pm 773$ \\
\hline
\end{tabular}




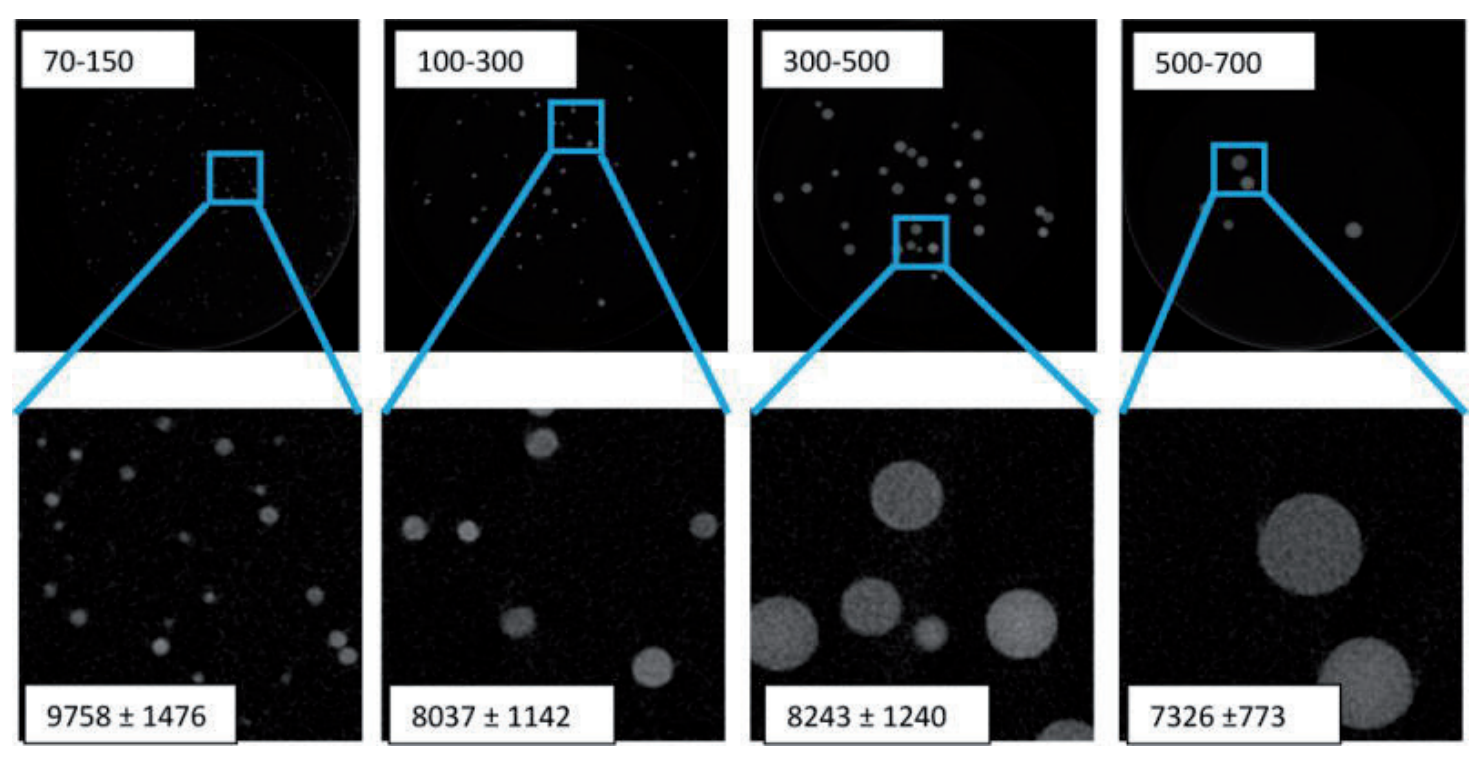

Figure 4.7 Comparison of iodinated bead (iBead II) size with their attenuation. The 70-150 mm bead has high attenuation than all other bead size $(\mathrm{p}<0.05)$.

\section{Doxorubicin loading and elution}

The bright red colored Dox solution was depleted within 3 hours of incubating with the radiopaque beads giving reddish beads with a Dox concentration of 40$80 \mathrm{mg} / \mathrm{ml}$ of beads similar to previously published for the raw beads $[58,79]$. Dox elution profiles for iodinated beads with two different size populations (100-300 $\mu$ and 300-500 $\mu$ ) and the smaller sized beads (100-300 $\mu \mathrm{m})$ exhibited slightly more release as previously reported (Figure 4.8) [79].

\section{Discussion}

In this study, we present a successful methodology for the synthesis of radiopaque PVA based microspheres (iBeads). The radiopacity of the microspheres was confirmed both by clinical CT and MicroCT. The hydroxyl functional group of the PVA microspheres were mildly activated with CDI and coupled with either 2,3,5-triiodobenzyl alcohol or trimethylenediamine. Diamine conjugated beads were iodinated with 2,3,5-triiodobenzoic acid (iBead II, Figure 4.2) under both 


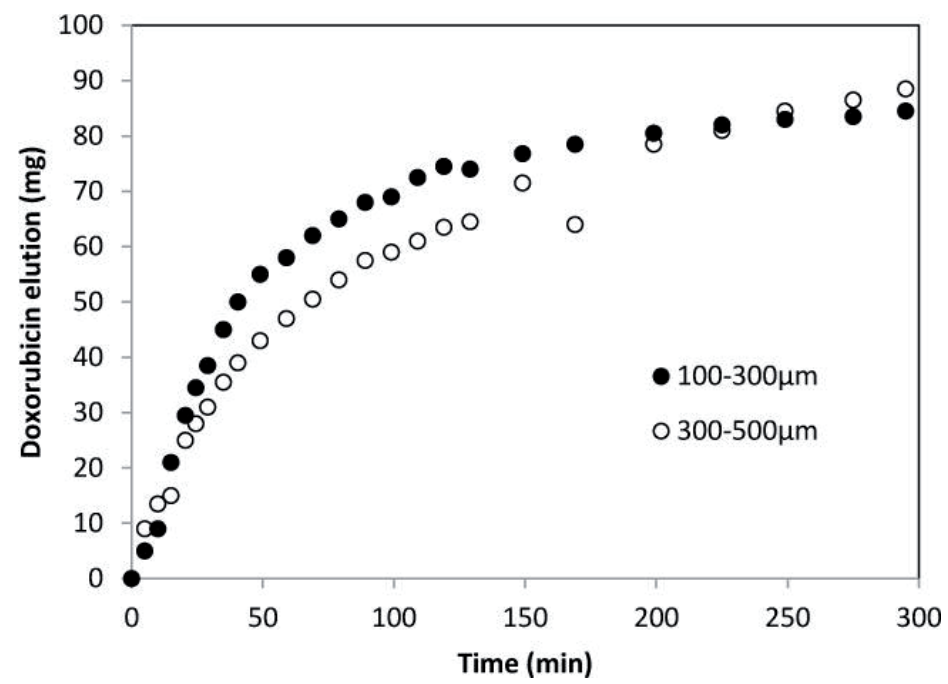

Figure 4.8 Doxorubicin elution from iodinated beads (iBead II) (100-300 mm and 300-500 mm).

DIC and CDI conditions. The diamine was used as spacer between the PVA bead hydroxyl group and the conjugating 2,3,5-triiodobenzoic acid. The completeness of the conjugation was monitored by the Ninhydrin test as well as by elemental analysis for iodine content. Under both conditions, on average the iodine content of iodinated beads achieved $45.6 \%$ in dried state according to elemental analysis results. Direct conjugation of the triiodobenzyl alcohol on the activated beads resulted in low conspicuity compared to the triiodobenzoic acid which is conjugated through a trimethylenediamine spacer. The spacer may provide less steric hindrance between the bulky activating reagent (carbonylimidazole) and the triiodobenzoic acid so that more trimethylenediamine spacer reacts with the activated bead thereby providing a suitable environment for more triiodobenzoic acid to conjugate to the beads. Therefore, the individual radiopaque beads made of triiodobenzoic acid through a trimethylenediamine spacer had $\sim 13$ times greater attenuation under microCT than radiopaque beads made from the corresponding benzyl alcohol. 
To examine the effect of bead size on iodine content and attenuation, the beads were sieved into different sizes (Table above \& Figure 4.7) and were tested for solid content, iodine content and microCT attenuation, respectively. Although the iodine content in dry beads decreased with decreasing bead size, the attenuation was greater due to high density of the smaller bead size.

Overall, the size of the iBead was smaller than the raw beads due to the hydrophobicity of the triiodobenzoic moiety which reduces the interaction of water molecules with the iBead [80].

Ideally, a chemoembolization agent is capable of providing occlusion of target vessels, high-capacity drug loading, selective delivery of the drug to the tumor, and real-time imaging feedback of the embolic particle and drug location with 2D and 3D X-ray imaging [81, 82]. Since all clinically available embolic agents are radiolucent, complications like non-target embolization may occur undetected with the embolic agent alone. For this reason, chemoembolization procedures currently rely on the injection of a mixture of the embolizing agent with X-ray contrast medium $[83,84]$. However, due to inadequate dispersion of the embolic material in the contrast media, the liquid media can traverse to the distal blood vessels more so than the embolic materials and as a result the fluoroscopic feedback this soluble contrast material provides misleading information about the location of the embolic material [85]. Instead, if the chemoembolic material has X-ray contrast property, the localization of embolic during and post embolization can be monitored and assessed in real-time with X-ray techniques. Such radiopaque embolic materials have been reported in the literature [6, 81, 82, 86-91], however, usually without drug loading capability. Recently, radiopaque beads and radiopaque Dox-eluting beads by mixing iodinated oil $\left(\right.$ Lipiodol $\left.^{\circledR}\right)$ with lyophilized PVA hydrogel beads were reported by our team and their use to chemoembolize 
swine liver and kidney was demonstrated $[57,73]$. However, due to the lack of off-the-shelf availability, this approach might have limited clinical use.

In summary, we report the synthesis of iBeads based on a commercial embolization microsphere (DC Bead) after mild activation of the bead with CDI and conjugating the activated bead with 2,3,5-triiodobenzoic acid using 1,3-diaminopropane as a spacer to alleviate steric hindrance. This radiopaque bead may be visualized under clinical CT and can load a similar level of Dox as the bland beads. It may be noted however, that the Dox loading capability is highly compromised when this radiopaque bead is subjected to high sterilization temperature. We speculate that the high sterilization temperature may cause the hydrophobic moiety to orient itself in such a way that to obscure the sulfonate group responsible for driving Dox into the bead. Therefore, alternative chemistry or sterilization technique is required to solve this problem.

\section{Conclusion}

Radiopaque drug-eluting beads were successfully synthesized by direct chemical conjugation of a radio-dense triiodobenzyl species on the commercial bead. These image-able embolic beads are visible under clinical imaging conditions which enable real-time feedback of bead location and drug localization during and after DEB-TACE. 


\section{References}

1. Chuang, V.P. and S. Wallace, Current status of transcatheter management of neoplasms. Cardiovasc. Interv. Radiol., 1980. 3(4): p. 256-265.

2. Loffroy, R., et al., Endovascular therapeutic embolization: an overview of occluding agents and their effects on embolised tissues. Curr. Vasc. Pharmacol., 2009. 7(2): p. 250-263.

3. Rosch, J., Antonovi.R, and C.T. Dotter, Selective vscoconstricto infusion in management of arterio-capillary gastronitestinal hemorrhage. Am. J. Roentgenol., 1972. 116(2): p. 279-\&.

4. $\quad$ Prochask.Jm, M.W. Flye, and Johnsrud.Is, Left gastric artery embolization for control of gastric bleeding complication. Radiol., 1973. 107(3): p. 521-522.

5. Baum, S. and M. Nusbaum, Control of gastrointestinal hemorrhage by selective mesenteric arterial infusion of vasopressin. Radiol., 1971. 98(3): p. 497-505.

6. Bartling, S.H., et al., First multimodal embolization particles visible on $x$-ray/computed tomography and magnetic resonance imaging. Invest. Radiol., 2011. 46(3): p. 178-86.

7. Newton, T.H. and J.E. Adams, Angiographic demonstration and nonsurgical embolization of spinal cord angioma. Radiol., 1968. 91(5): p. 873-876.

8. Luessenh.Aj, et al., Clinical evaluation of artificial embolization in management of large cerebral arteriovenous malformations. J. Neurosurgery, 1965. 23(4): p. 400-417.

9. Chang, S.D., et al., Multimodality treatment of giant intracranial arteriovenous malformations (Reprinted from Archival article vol 53, pg 1, 2003). Neurosurgery, 2007. 61(1): p. 432-442.

10. Luessenhop, A.J. and P.H. Mujica, Embolization of segments of the circles of Willis and adjacent branches for management of certain arteriovenous malformations. J. Neurosurgery, 1981. 54(5): p. 573-582.

11. Doppman, J.L., G. Dichiro, and A. Ommaya, Obliteration of spinal cord arteriovenous malformation by percutaneous embolisation. Lancet, 1968. 1(7540): p. 477.

12. Beaujeux, R., et al., Trisacryl gelatin microspheres for therapeutic embolization .2. Preliminary clinical evaluation in tumors and arteriovenous malformations. Am. J. Neuroradiol., 1996. 17(3): p. 541-548.

13. Marshburn, P.B., M.L. Matthews, and B.S. Hurst, Uterine artery embolization as a treatment option for uterine myomas. Obstet. Gynecol. Clin. North Am., 2006. 33(1): p. 125-144.

14. Manelfe, C., et al., Transfemoral catheter embolization in intracranial meningiomas. Rev. Neurol., 1973. 128(5): p. 339-351.

15. Rand, T., et al., Arterial embolization of unresectable hepatocellular carcinoma with use of microspheres, lipiodol, and cyanoacrylate. Cardiovas. Interv. Radiol., 2005. 28(3): p. 313-318.

16. Krysl, J. and D.A. Kumpe, Embolization agents: A review. Tech. Vasc. Interv. Radiol., 2000. 3(3): p. 158-161.

17. Subramanian, V., et al., Synthesis and characterization of polyvinylpyrrolidine assisted tantalum pentoxide films. Thin Solid Films, 2008. 516(15): p. 4784-4792.

18. Kirchhoff, T.D., et al., Transarterial chemoembolization using degradable starch microspheres and iodized oil in the treatment of advanced hepatocellular carcinoma: evaluation of tumor response, toxicity, and survival. Hepatobiliary Pancreat. Dis. Int., 2007. 6(3): p. 259-266.

19. Stampl, S., et al., Inflammation and recanalization of four different spherical embolization agents in the porcine kidney model. J. Vasc. Interv. Radiol., 2008. 19(4): p. 577-586.

20. Rao, V.R.K., et al., Hydrolyzed microspheres from cross linked polymethyl methacrylate (hydrogel)-a new embolic material for interventional neuroradiology. J. Neuroradiol., 1991. 18(1): p. 61-69. 
21. Jayakrishnan, A., et al., Hydrogel microspheres from crosslinked poly(methyl methacrylatesynthesis and biocompatibility studies. Bull. Mater. Sci., 1989. 12(1): p. 17-25.

22. Ducmauger, A., J.P. Benoit, and F. Puisieux, Preparation and characterization of cross-linked human serum albumin microcapsules containing 5-fluorouracil. Pharm. Acta Helv., 1986. 61(4): p. 119-124.

23. Benita, S., O. Zouai, and J.P. Benoit, 5-Fluorouracil carnauba wax microspheres for chemoembolization-an in vitro evaluation. J. Pharm. Sci., 1986. 75(9): p. 847-851.

24. Spenlehauer, G., M. Veillard, and J.P. Benoit, Formation and characterization of cisplatin loaded poly(d,l-lactide) microspheres for chemoembolization. J. Pharm. Sci., 1986. 75(8): p. 750-755.

25. Laurent, A., et al., Trisacryl gelatin microspheres for therapeutic embolization, I: development and in vitro evaluation. Am. J. Neuroradiol., 1996. 17(3): p. 533-540.

26. Rodiek, S.O., A. Stolzle, and C.B. Lumenta, Preoperative embolization of intracranial meningiomas with Embosphereâ microspheres. Minim. Invasive Neurosurgery, 2004. 47(5): p. 299-305.

27. Choi, J.W., et al., Comparison of drug release and pharmacokinetics after transarterial chemoembolization using diverse lipiodol emulsions and drug-eluting beads. Plos One, 2014. 9(12).

28. Lee, H.B., et al., Preparation of gradient polymer surface and their pluripotent biomedical applications. Polym. Korea, 2005. 29(5): p. 423-432.

29. Lopez-Benitez, R., et al., Analysis of nontarget embolization mechanisms during embolization and chemoembolization procedures. Cardiovasc. Interv. Radiol., 2009. 32(4): p. 615-622.

30. Malagari, K., Drug-eluting particles in the treatment of HCC: chemoembolization with doxorubicin-loaded DC Bead (TM). Expert Rev. Anticancer Ther., 2008. 8(10): p. 16431650 .

31. Grosso, M., et al., Transarterial chemoembolization for hepatocellular carcinoma with drugeluting microspheres: preliminary results from an Italian multicentre study. Cardiovasc. Interv. Radiol., 2008. 31(6): p. 1141-1149.

32. Jordan, O., et al., Comparative study of chemoembolization loadable beads: in vitro drug release and physical properties of DC Bead and Hepasphere loaded with doxorubicin and irinotecan. J. Vasc. Interv. Radiol., 2010. 21(7): p. 1084-1090.

33. Llovet, J.M. and J. Bruix, Systematic review of randomized trials for unresectable hepatocellular carcinoma: chemoembolization improves survival. Hepatology, 2003. 37(2): p. 429-42.

34. Chang, Y., et al., A highly stable non biofouling surface with well-packed grafted zwitterionic polysulfobetaine for plasma protein repulsion. Langmuir, 2008. 24(10): p. 5453-5458.

35. Bruix, J., et al., New aspects of diagnosis and therapy of hepatocellular carcinoma. Oncogene, 2006. 25(27): p. 3848-3856.

36. Varela, M., et al., Treatment of hepatocellular carcinoma: is there an optimal strategy? Cancer Treat Rev., 2003. 29(2): p. 99-104.

37. Bruix, J., et al., Focus on hepatocellular carcinoma. Cancer Cell, 2004. 5(3): p. 215-219.

38. Abada, H.T. and J. Golzarian, Gelatin sponge particles: handling characteristics for endovascular use. Tech. Vasc. Interv. Radiol., 2007. 10(4): p. 257-60.

39. Padhya, K.T., J.A. Marrero, and A.G. Singal, Recent advances in the treatment of hepatocellular carcinoma. Curr. Opin. Gastroenterol., 2013. 29(3): p. 285-292.

40. Singal, A.G. and J.A. Marrero, Recent advances in the treatment of hepatocellular carcinoma. Curr. Opin. Gastroenterol., 2010. 26(3): p. 189-195. 
41. Himoto, T., et al., Recent advances in radiofrequency ablation for the management of hepatocellular carcinoma. Hepat. Mon., 2012. 12(10). e5945.

42. Minami, Y. and M. Kudo, Radiofrequency ablation of hepatocellular carcinoma: current status. World J. Radiol., 2010. 2(11): p. 417-424.

43. Forner, A., et al., Treatment of hepatocellular carcinoma. Crit. Rev. Oncol. Hema., 2006. 60(2): p. 89-98.

44. Llovet, J.M., et al., Arterial embolization or chemoembolization versus symptomatic treatment in patients with unresectable hepatocellular carcinoma: a randomized controlled trial. Lancet, 2002.359(9319): p. 1734-1739.

45. Sikander, A., et al., Transarterial chemoembolization in a patient with recurrent hepatocellular carcinoma and portal vein thrombosis $-A$ case report and review of the literature. Am. J. Clin. Oncol., 2005. 28(6): p. 638-639.

46. O'Toole, D. and P. Ruszniewski, Chemoembolization and other ablative therapies for liver metastases of gastrointestinal endocrine tumours. Best Pract. Res. Clin. Gastroenterol., 2005. 19(4): p. 585-594.

47. Abraham, S., et al., Formation of transition metall-doxorubicin complexes inside liposomes. Biochim. Biophys. Acta-Biomembr., 2002. 1565(1): p. 41-54.

48. Allen, T., Ligand-targeted therapeutics in anticancer therapy. Nat. Rev. Cancer, 2002. 2(10): p. 750-763.

49. Arap, W., et al., Steps toward mapping the human vasculature by phage display. Nature Med., 2002. 8(2): p. 121-127.

50. Avgoustakis, K., et al., PLGA-mPEG nanoparticles of cisplatin: in vitro nanoparticle degradation, in vitro drug release and in vivo drug residence in blood properties. J. Control. Release, 2002. 79(1-3): p. 123-135.

51. Balazs, M., et al., Blood dendritic cells interact with splenic marginal zone B cells to initiate T-independent immune responses. Immunity, 2002. 17(3): p. 341-352.

52. Barenholz, Y., Relevancy of drug loading to liposomal formulation therapeutic efficacy. J. Liposome Res., 2003. 13(1): p. 1-8.

53. Basile, A., et al., Trisacryl gelatin microspheres versus polyvinyl alcohol particles in the preoperative embolization of bone neoplasms. Cardiovasc. Interv. Radiol., 2004. 27(5): p. 495-502.

54. Bi, Y., et al., Actively targeted nanoparticles for drug delivery to tumor. Curr. Drug Metab., 2016. 17(8): p. 763-782.

55. Bico, J., U. Thiele, and D. Quere, Wetting of textured surfaces. Colloids Surf., A Physicochem. Eng. Asp., 2002. 206(1-3): p. 41-46.

56. Chun, H.J., et al., An experimental study for syndiotactic polyvinyl alcohol spheres as an embolic agent: can it maintain spherical shape in vivo? Biomed. Mater. Eng., 2014. 24(4): p. 17431750 .

57. Dreher, M.R., et al., Radiopaque drug-eluting beads for transcatheter embolotherapy: experimental study of drug penetration and coverage in swine. J. Vasc. Interv. Radiol., 2012. 23(2): p. 257-264.

58. Lewis, A.L., et al., Doxorubicin eluting beads-1: effects of drug loading on bead characteristics and drug distribution. J. Mater. Sci. Mater. Med., 2007. 18(9): p. 1691-1699.

59. Lewis, A.L., et al., DC bead: in vitro characterization of a drug-delivery device for transarterial chemoembolization. J. Vasc. Interv. Radiol. 2006. 17(2): p. 335-342.

60. Lewis, A.L. and R.R. Holden, DC bead embolic drug-eluting bead: clinical application in the locoregional treatment of tumours. Expert Opin. Drug Deliv., 2011. 8(2): p. 153-169. 
61. Lewis, A.L. and M.R. Dreher, Locoregional drug delivery using image-guided intra-arterial drug eluting bead therapy. J. Control. Release, 2012. 161(2): p. 338-350.

62. Lewandowski, R.J., et al., Transcatheter intraarterial therapies: rationale and overview. Radiology, 2011. 259(3): p. 641-657.

63. Liapi, E. and J.F.H. Geschwind, Transcatheter arterial chemoembolization for liver Cancer: is it time to distinguish conventional from drug-eluting chemoembolization? Cardiovasc. Interv. Radiol., 2011. 34(1): p. 37-49.

64. Llovet, J.M. and J. Bruix, Unresectable hepatocellular carcinoma: meta-analysis of arterial embolization. Radiology, 2004. 230(1): p. 300-301.

65. Lo, C.M., et al., Randomized controlled trial of transarterial lipiodol chemoembolization for unresectable hepatocellular carcinoma. Hepatology, 2002. 35(5): p. 1164-1171.

66. Yamada, R., et al., Hepatic artery embolization in 120 patients with unresectable hepatoma. Radiology, 1983. 148(2): p. 397-401.

67. Konno, T., et al., Effect of arterial administration of high molecular agent SMAMCS with lipid lymphgraphic agent on hepatoma-a preliminary report. Eur. J. Cancer Clin. Oncology, 1983. 19(8): p. 1053-\&.

68. Stampfl, U., et al., Midterm results of uterine artery embolization using narrow-size calibrated embozene microspheres. Cardiovasc. Interv. Radiol., 2011. 34(2): p. 295-305.

69. Sadick, M., et al., Application of DC beads in hepatocellular carcinoma: clinical and radiological results of a drug delivery device for transcatheter superselective arterial embolization. Onkologie, 2010. 33(1-2): p. 31-37.

70. Aliberti, C., et al., Transarterial chemoembolization of metastatic colorectal carcinoma to the liver adopting DC bead, drug-eluting bead loaded with irinotecan: results of a phase II clinical study. Anticancer Res., 2011. 31(12): p. 4581-4587.

71. Fiorentini, G., et al., Intraarterial hepatic chemoembolization (TACE) of liver metastases from ocular melanoma with slow release irinotecan-eluting beads. Early results of a phase II clinical study. In Vivo, 2009. 23(1): p. 131-137.

72. Eichler, K., et al., First human study in treatment of unresectable liver metastases from colorectal cancer with irinotecan-loaded beads (DEBIRI). Int. J. Oncol., 2012. 41(4): p. 1213-1220.

73. Sharma, K.V., et al., Development of "Imageable" Beads for transcatheter embolotherapy. J. Vasc. Interv. Radiol., 2010. 21(6): p. 865-876.

74. Fiorentini, G., et al., Transarterial chemoembolization of metastatic colorectal carcinoma $(M C R C)$ to the liver adopting polyvinyl alcohol microspheres (PAM) loaded with irinotecan compared with FOLFIRI (CT): evalution at two years of a phase III clinical trial. Ann. Oncol., 2010. 21: p. 191-191.

75. Gonzalez, M.V., et al., Doxorubicin eluting beads-2: methods for evaluating drug elution and in vitro : in vivo correlation. J. Mat. Sci. Mat. Med., 2008. 19(2): p. 767-775.

76. Tang, Y., et al., Evaluation of irinotecan drug-eluting beads: a new drug-device combination product for the chemoembolization of hepatic metastases. J. Control. Release, 2006. 116(2): p. E55-E56.

77. Taylor, R.R., et al., Irinotecan drug eluting beads for use in chemoembolization: in vitro and in vivo evaluation of drug release properties. Eur. J. Pharm. Sci., 2007. 30(1): p. 7-14.

78. Kaiser, E., et al., Color test for detection of free terminal amino groups in solid phase synthesis of peptides. Anal. Biochem., 1970. 34(2): p. 595-\&.

79. Lewis, A.L., et al., Pharmacokinetic and safety study of doxorubicin-eluting beads in a porcine model of hepatic arterial embolization. J. Vasc. Interv. Radiol., 2006. 17(8): p. 1335-1343. 
80. Mawad, D., et al., Synthesis and characterization of radiopaque iodine-containing degradable PVA hydrogels. Biomacromolecules, 2008. 9(1): p. 263-268.

81. Okamura, M., et al., Synthesis and properties of radiopaque polymer hydrogels: polyion complexes of copolymers of acrylamide derivatives having triiodophenol and carboxyl groups and p-styrene sulfonate and polyallylamine. J. Mol. Struct., 2000. 554(1): p. 35-45.

82. Jayakrishnan, A., et al., Preparation and evaluation of radiopaque hydrogel microspheres based on phemaiothalamic acid and phemaiopanoic acid as particulate emboli. J. Biomed. Mater. Res., 1990. 24(8): p. 993-1004.

83. Bendszus, M., et al., Efficacy of trisacryl gelatin microspheres versus polyvinyl alcohol particles in the preoperative embolization of meningiomas. Am. J. Neuroradiol., 2000. 21(2): p. 255-261.

84. Bendszus, M., et al., MR imaging-and MR spectroscopy-revealed changes in meningiomas for which embolization was performed without subsequent surgery. Am. J. Neuroradiol., 2000. 21(4): p. 666-669.

85. Gaba, R.C., et al., Ethiodized oil uptake does not predict doxorubicin drug delivery after chemoembolization in VX2 liver tumors. J. Vasc. Interv. Radiol., 2012. 23(2): p. 265-273.

86. Thanoo, B.C. and A. Jayakrishnan, Barium sulfate loaded p(HEMA) microspheres as artificial emboli-preparation and properties. Biomaterials, 1990. 11(7): p. 477-481.

87. Jayakrishnan, A., W.A. Knepp, and E.P. Goldberg, Casein microspheres-preparation and evaluation as a carrier for controlled drug delivery. Int. J. Pharm., 1994. 106(3): p. 221-228.

88. Thanoo, B.C. and A. Jayakrishnan, Tantalum loaded silicone microspheres as particulate emboli. J. Microencapsul., 1991. 8(1): p. 95-101.

89. Horak, D., et al., Hydrogels in endovascular embolization. 3. Radiopaque spherical particles, their preparation and properties. Biomaterials, 1987. 8(2): p. 142-145.

90. Thanoo, B.C. and A. Jayakrishnan, Radiopaque hydrogel microspheres. J. Microencapsul., 1989. 6(2): p. 233-244.

91. Hagit, A., et al., Synthesis and characterization of dual modality (CT/MRI) core-shell microparticles for embolization purposes. Biomacromolecules, 2010. 11(6): p. 1600-1607. 


\section{Chapter 5}

\section{Synthesis, characterization, and imaging of radiopaque bismuth beads for image-guided transarterial embolization}

Ayele H. Negussie ${ }^{1,6^{*}}$, Quirine M. B. de Ruiter ${ }^{1 *}$, Hugh Britton ${ }^{2}$, Danielle R.

Donahue $^{3}$, Quentin Buffie ${ }^{2}$, Young-Seung Kim ${ }^{4}$, William F. Pritchard ${ }^{1}$, Chrit Moonen ${ }^{5}$, Gert Storm ${ }^{6,7,8}$, Andrew L. Lewis ${ }^{2}$, Bradford J. Wood ${ }^{1,9}$

${ }^{1}$ Center for Interventional Oncology, Radiology and Imaging Sciences, Clinical Center, National Institutes of Health, 9000 Rockville Pike, Bethesda, MD, USA

${ }^{2}$ Biocompatibles UK Ltd, (a BTG, Now Boston Scientific Company), Lakeview, Riverside Way, Watchmoor Park, Camberley, GU15 3YL, Surrey, UK

${ }^{3}$ Mouse Imaging Facility, National Institute of Neurological Disorders and Stroke, National Institutes of Health, Bethesda, MD, USA

${ }^{4}$ Radiation Oncology Branch, National Cancer Institute, National Institutes of Health, Bethesda, MD, USA

${ }^{5}$ Center for Imaging Sciences, Imaging Division, University Medical Center Utrecht, Utrecht, 3584 CX, The Netherlands

${ }^{6}$ Department of Biomaterials Science and Technology, University of Twente, Enschede, The Netherlands

${ }^{7}$ Department of Pharmaceutics, Utrecht University, Utrecht, The Netherlands

${ }^{8}$ Department of Surgery, Yong Loo Lin School of Medicine, National University of Singapore, Singapore, Singapore

${ }^{9}$ Center for Interventional Oncology, Radiology and Imaging Sciences, Clinical Center, Center for Cancer Research, National Cancer Institute, National Institutes of Health, Bethesda, MD, USA.

Published in: Sci. Rep., (11) 2021

* Authors contributed equally 


\section{Abstract}

This study aimed to synthesize and characterize imageable beads using bismuth as the radiopacifier that could be distinguished from iodine contrast based upon the difference in the binding energy of k-shell electrons (k-edge). Radiodense bismuth beads were successfully synthesized some with uniform bismuth distribution across the beads. The beads were spherical and could be infused through clinical microcatheters. The bismuth beads could be imaged with clinical dual-energy computed tomography (CT), where iodine-based contrast could be distinguished from the microspheres. The ability to separate iodine from bismuth may enhance the diagnostic information acquired on follow-up CT, identifying the distribution of the embolic beads separately from the contrast. Furthermore, with sequential use of iodine- and bismuth-based beads, the two radiopaque beads could be spatially distinguished on imaging, which may enable the development of dual drug delivery and dual tracking. 


\section{Introduction}

Embolic microspheres (beads) are used to occlude the vascular supply of tumors and may be loaded with chemotherapy drugs, which then elute from the microspheres and diffuse into the tissue. These drug-eluting beads are used for locoregional therapy, particularly for treating patients with unresectable hepatocellular carcinoma[1]. Embolic beads are typically made of inert hydrophilic materials with elastic properties and spherical geometry. These beads are generally nonradiopaque but can be rendered radiopaque by incorporation of a radioabsorber, such as iodine, zinc, tantalum, bismuth, or barium[2-6]. The development and use of imageable beads can inform direct real-time feedback during the embolization procedure, or on follow-up imaging[7, 8].

Recently, we have developed a radiopaque embolic bead through covalent attachment of iodinated groups onto the backbone of polyvinyl alcohol-coacrylamido-2-methylpropane sulfonate precursor microspheres $[2,9,10]$. The radiopacity of the beads during delivery or follow up can facilitate identification, localization, and distribution of undertreated tumor[11, 12]. It may also enable estimation of doxorubicin dose and spatial distribution in the liver[13]. One limitation of iodinated beads, however, is the challenge in distinguishing them from iodinated contrast agents using imaging based on X-ray absorption, i.e., fluoroscopy, cone beam CT, or CT imaging. During embolization, embolic agents (both radiopaque and non-radiopaque beads) are mixed with contrast agents to improve suspension and handling, and to estimate dosimetry and delivery of the beads to the tumor. Contrast may be somewhat retained, although intravascular contrast washes out over time[14]. Similarly, a contrast-enhanced CT scan after bead delivery may not normally differentiate among intravascular liquid contrast, iodinated beads, and contrast in stained parenchyma. 
Attaching a radiopaque element with a higher atomic number, $\mathrm{Z}$, and a different k-edge from iodine, may enable material decomposition and differentiation of a novel bead from iodine in contrast or from iodinated radiopaque beads using dualenergy CT (DECT) [15]. In DECT, two CT scans are simultaneously acquired at different energies, a low-energy image, e.g., $80 \mathrm{keV}$, and a higher-energy image, e.g., $140-150 \mathrm{keV}$, that can be processed to generate additional datasets that enable material decomposition. Bismuth has a k-edge of $90.5 \mathrm{keV}$ compared to $33.2 \mathrm{keV}$ for iodine and thus has the potential to be differentiated from iodine using DECT.

The purpose of this study was to synthesize and characterize novel imageable beads using bismuth as the radiopacifier. A secondary goal was to assess the ability to spatially differentiate the bismuth beads from iodine-based contrast agents using DECT imaging, as a requisite step towards dual tracked drug vectors in embolization therapies.

\section{Materials and Methods}

\section{Materials}

All reagents were purchased from commercial sources and used without further purifications. Acrylamido polyvinyl alcohol-co-acrylamido-2-methylpropane sulfonate microspheres, dried to a free-flowing powder and untainted, were obtained from Biocompatibles UK Ltd, (a BTG now Boston Scientific company, Farnham, UK). $\mathrm{Bi}\left(\mathrm{CF}_{3} \mathrm{SO}_{3}\right)_{3}$ was chosen as bismuth salt and was obtained from Sigma-Aldrich (Milwaukee, WI). SEM and EDX were used to evaluate surface smoothness and bismuth distribution across the bead, respectively (EMSL Analytical, Inc, Cinnaminson, NJ). Elemental composition was performed by Robertson Microlit Laboratories (Ledgewood, NJ). Material decomposition 
techniques were used to determine whether bismuth and iodine could be distinguished using DECT.

\section{Bismuth Beads Synthesis}

\section{General}

Reaction progress was monitored by reverse-phase HPLC (Rp-HPLC) using a Beckman System Gold HPLC (Fullerton, CA) equipped with a 126 solvent module and $168 \mathrm{UV}$ detector $(\lambda=254 \mathrm{~nm})$ controlled by 32 Karat software and Beckman Ultrasphere $\mathrm{C}_{18}$ column (ODS, 4.6 x $250 \mathrm{~mm}, 5 \mu \mathrm{m}$ ), mobile phase: (A) $90 \%(0.1 \% \mathrm{TFA}$ in water); (B) $10 \%$ (0.1\% TFA in acetonitrile); $0-5 \mathrm{~min}$, $10-90 \% \mathrm{~B}, 5-15 \mathrm{~min}, 90 \% \mathrm{~B}, 15-25 \mathrm{~min}$. The flow rate was set to $1 \mathrm{~mL} / \mathrm{min}$. TLC was carried out on Merck silica gel 60 TLC plates $\mathrm{F}_{254}$ and visualized by UV at $254 \mathrm{~nm}$. Column chromatography was performed using silica gel 60 (70-230 mesh). ${ }^{1} \mathrm{H}$ and ${ }^{13} \mathrm{C}$ were NMR recorded on a Bruker Avance 300 instrument with a deuterated solvent. Mass spectra were recorded with a Waters (Waltham, MA USA) LCT Premiere ESI TOF mass spectrometer. The instrument was operated in positive ion ESI mode at a mass resolution of 10,000.

\section{Synthesis of Macrocycle}

\section{4-Formylbenzyl-DO3A-tris-(t-Bu ester) (2)}

The titled compound (2) was synthesized as described by Mondal et al. [16] with slight modification. To a solution of DO3A-t-Bu-ester (1) (5 g, 9.7 $\mathrm{mmol}$ ) and 4-(bromomethyl)benzaldehyde $(1.93 \mathrm{~g}, 9.7 \mathrm{mmol})$ in anhydrous $N$, $N$-dimethylformamide (DMF) $(60 \mathrm{~mL})$ was added $\mathrm{K}_{2} \mathrm{CO}_{3}(1.6 \mathrm{~g}, 11.64 \mathrm{mmol})$. The reaction was stirred at room temperature for $4 \mathrm{~h}$. The reaction progress was monitored by Rp-HPLC as described in the general method. The reaction mixture was filtered, and the solvent was removed. The product was purified by column chromatography (silica gel, $\mathrm{MeOH}$ :DCM from 0-10\%) to give (2) (4.7 g, 76\%) 
dull yellow powder. ${ }^{1} \mathrm{HNMR}\left(\mathrm{CDCl}_{3}, 300 \mathrm{MHz}\right): \delta$ ppm $1.5\left(27 \mathrm{H}, \mathrm{s},{ }^{\mathrm{t}} \mathrm{Bu}\right)$, 2.2-2.9 (16H, m, $\left.\mathrm{CH}_{2}\right), 3.0-3.1\left(6 \mathrm{H}\right.$, brs, $\left.\mathrm{CH}_{2} \mathrm{COOtBu}\right), 3.2\left(2 \mathrm{H}, \mathrm{s}, \mathrm{CH}_{2}\right)$, $7.7(2 \mathrm{H}, \mathrm{d}, \mathrm{J}=8 \mathrm{~Hz}, \mathrm{Ph}), 7.9(2 \mathrm{H}, \mathrm{d}, \mathrm{J}=8 \mathrm{~Hz}, \mathrm{Ph}), 10.0$ (s, HCO); ${ }^{13} \mathrm{C}$ NMR $\left(\mathrm{CDCl}_{3}, 75 \mathrm{MHz}\right): \delta$ ppm 27.6, 49.7, 55.4, 55.7, 59.1, 82.2, 82.6, 129.7, 130.8, 135.3, 144.4, 172.4, 173.2, 191.5. MS-ESI: $m / z(\%)=633.4(100)\left[(\mathrm{M}+\mathrm{H})^{+}\right]$, $\operatorname{HRMS}\left[(\mathrm{M}+\mathrm{H})^{+}\right]=$calcd. for: $\left[\mathrm{C}_{34} \mathrm{H}_{57} \mathrm{~N}_{4} \mathrm{O}_{7}\right]^{+}$663.4220; found 663.4227 .

\section{4-Formylbenzoyl-DO3A-tris-(t-Bu ester) (3)}

4-Formylbenzoyl-DO3A-tris-(t-Bu ester) (3) was made from 4-formylbenzoic acid (4-FBA) (20.91g, $139.14 \mathrm{mmol})$. 4-Formylbenzoyl chloride was synthesized by the method of Iserloh et al. [17]. 4-Formylbenzoyl chloride was coupled with the DO3A-tris-(t-Bu ester) $(60.01 \mathrm{~g}, 92.76 \mathrm{mmol})$ as described by Bhakuni et. al. [18]. The resulting reaction mixture was purified by column chromatography (Biotage Isolera One chromatography system on $340 \mathrm{~g}$ snap columns, Biotage Sweden) with a gradient solvent system of ethyl acetate: methanol; 100\%:0\% to $95 \%: 5 \%$ to give the desired product, 3 as pale yellow viscous syrup $(55.33 \mathrm{~g}$, 73.4\% yield and $97 \%$ HPLC purity); ( ${ }^{1} \mathrm{HNMR}\left(\mathrm{CDCl}_{3}, 500 \mathrm{MHz}\right): \delta \mathrm{ppm}$ $1.41(9 \mathrm{H}, \mathrm{s}), 1.45(9 \mathrm{H}, \mathrm{s}), 1.47(9 \mathrm{H}, \mathrm{s}), 2.70-2.68(2 \mathrm{H}, \mathrm{m}), 2.80-2.73(6 \mathrm{H}, \mathrm{m})$, $2.91(2 \mathrm{H}, J 6.5 \mathrm{~Hz}), 3.03(2 \mathrm{H}, \mathrm{t}, J 5.3 \mathrm{~Hz}), 3.07(2 \mathrm{H}, \mathrm{s}), 3.31(2 \mathrm{H}, \mathrm{s}), 3.32(2 \mathrm{H}$, s), $3.62(2 \mathrm{H}, J 6.5 \mathrm{~Hz}), 3.79(2 \mathrm{H}, \mathrm{t}, J 5.3 \mathrm{~Hz}), 7.55(2 \mathrm{H}, \mathrm{d}, J 8.1 \mathrm{~Hz}), 7.90(2 \mathrm{H}$, $\mathrm{d}, J 8.1 \mathrm{~Hz}), 10.04(1 \mathrm{H}, \mathrm{s}) ;{ }^{13} \mathrm{C} \mathrm{NMR}\left(\mathrm{CDCl}_{3}, 125 \mathrm{MHz}\right): \delta \mathrm{ppm} ; 28.16,28.24$, $44.67,48.87,52.00,52.32,52.82,53.05,53.52,54.71,54.98,57.64,58.16$, , $80.89,80.94,80.98,127.16,129.81,136.38,143.32$, 170.49, 170.64, 170.76, 170.80, 191.62 . 


\section{Deprotection of Compound 2 and 3 \\ DOTA-4-formylbenzyl (4)}

Compound $2(9.2 \mathrm{~g}, 14.6 \mathrm{mmol})$ was treated with $4 \mathrm{~N} \mathrm{HCl}$ in dioxane $(450 \mathrm{~mL})$ by the method described by Muhlemann et al. [19] to give DOTA-4-formylbenzyl (4). $8 \mathrm{~g}$ (qualitative yield) of dull white powder was obtained. MS-ESI: $m / z(\%)$ $=465.2(100)\left[(\mathrm{M}+\mathrm{H})^{+}\right], \operatorname{HRMS}\left[(\mathrm{M}+1)^{+}\right]$: calcd. for: $\left[\mathrm{C}_{22} \mathrm{H}_{33} \mathrm{~N}_{4} \mathrm{O}_{7}\right]^{+} 465.2345$; found 465.2349 .

\section{DOTA-4-formylbenzoyl (5)}

Compound $\mathbf{3}$ was treated using a similar method as described in compound $\mathbf{4}$ to give DOTA-4-formylbenzoyl (5) (yield, 87\%, purity confirmed by HPLC).

\section{Acetalation of the Beads (6)}

Method I Aromatic linker of compound $\mathbf{4}$

Method Ia : DMSO as a reaction solvent

$1 \mathrm{~g}$ of beads (polyvinyl alcohol-co-acrylamido-2-methylpropane sulfonate precursor microspheres, Biocompatibles UK Ltd, (BTG/Boston Scientific), Farnham, UK) were swollen in $35 \mathrm{~mL}$ of anhydrous DMSO followed by adding $1 \mathrm{~g}$ of compound $4(2.15 \mathrm{mmol})$. The resulting mixture was stirred for 30 minutes to distribute the macrocycle compound $\mathbf{4}$ into the swollen beads uniformly. Then, $2.2 \mathrm{~mL}$ of methanesulfonic acid was added dropwise from a syringe through a 30 G, 1/2-inch needle, and the reaction mixture was stirred at a lower stirrer setting under $\mathrm{N}_{2}$ at $50^{\circ} \mathrm{C}$ for $26 \mathrm{~h}$. The progress and the reaction rate were monitored with HPLC, every two hours for the first eight hours and then at 24 and $26 \mathrm{~h}$, for the consumption of macrocyclic compound 4 . The reaction mixture was filtered. The filtrate was washed with DMSO $(10 \mathrm{~mL})$, then with copious amount of saturated sodium bicarbonate to completely remove the acid (monitored with 
litmus paper) and finally with deionized water. The acetalated beads (6) were used for the subsequent reaction.

\section{Method Ib: DMF as a reaction solvent}

Acetalation of the beads was repeated as described in a), except that the DMSO solvent was replaced with DMF solvent.

\section{Method II: Aromatic linker of compound 5}

Acetalation of the beads with compound 5 was performed in accordance with the method of Duran et al. [10] with the following adaptations: NMP ( $N$-methyl pyrrolidinone) was the solvent used, and the reactions were performed at 55 to 65 ${ }^{\circ} \mathrm{C}$ for up to $40 \mathrm{~h}$, monitored with HPLC for the consumption of the macrocycle. The liquid phase was removed, and the filtered beads were washed with NMP (90 $\mathrm{mL}$ ), then suspended in water and neutralized with $0.1 \mathrm{M}$ sodium carbonate to $\mathrm{pH}$ 8.5. The beads were then washed with water $(90 \mathrm{~mL})$ and NMP $(250 \mathrm{~mL})$ followed by neat NMP (150 mL). The acetalated beads (7) were used directly in the subsequent reaction.

\section{Chelation of Bismuth to the Acetalated Beads}

Acetalation of the beads with compound $\mathbf{4}$ was performed with two different reaction solvents: dimethyl sulfoxide (DMSO) and dimethylformamide (DMF)

\section{Method I Bead (6):}

The acetalated beads (6) (Synthetic Scheme 1) were resuspended in deionized water at a $\mathrm{pH}$ of $8-9$ using $0.1 \mathrm{~N}$ of sodium bicarbonate, and $\mathrm{Bi}\left(\mathrm{CF}_{3} \mathrm{SO}_{3}\right)_{3}(4$ mmol) was added and stirred at $80^{\circ} \mathrm{C}$ for $1 \mathrm{~h}$. The final beads were filtered and washed with an aqueous solution of sodium bicarbonate $(0.1 \mathrm{M})$ and finally with copious amounts of deionized water to remove residual bicarbonate (monitored 
by litmus paper). The complete removal of bismuth salt was confirmed using an aqueous solution of potassium iodide (1\%,w/v) as reported in the literature [20].

\section{Method II Beads (7)}

Acetalated beads (7) were suspended in anhydrous NMP (30 mL) under $\mathrm{N}_{2}$. Pyridine $(0.54 \mathrm{~mL})$ was added, then the mixture was warmed to $55^{\circ} \mathrm{C}$, followed by addition of bismuth trifluromethanesulfonate $(2.455 \mathrm{~g}, 1.5 \mathrm{eq}$.$) . The resulting$ suspension was stirred overnight at $55^{\circ} \mathrm{C}$ under $\mathrm{N}_{2}$. The liquid portion was removed, and the filtered beads were washed with NMP $(250 \mathrm{~mL})$. The beads were then suspended in phosphate buffered saline, neutralized to $\mathrm{pH} 7$ with $0.1 \mathrm{M}$ $\mathrm{Na}_{2} \mathrm{CO}_{3}$ and then washed with phosphate buffered saline $(250 \mathrm{~mL})$.

\section{Sterilization}

Bulk beads were dispensed into $20 \mathrm{~mL}$ Schott FIOLAX clear glass vials and sealed with bromobutyl Fluro Tec injection stoppers and FOTO caps and autoclaved at $121{ }^{\circ} \mathrm{C}$ for 30 minutes as reported previously[10].

\section{Characterization of Bismuth Beads \\ Physical Properties of Beads}

The solid content of the hydrated bead, bismuth concentration of hydrated bead, and the bismuth content of the dry beads of 7 were determined as described previously [10] (see SI). Both dry bismuth beads (6 and 7) were analyzed for their elemental composition by Robertson Microlit Laboratories (Ledgewood, NJ).

\section{Microscopic Evaluation}

Beads (7) were sieved into four sizes ranging between 100-600 $\mathrm{mm}$ in diameter and evaluated under light microscopy for surface deformation and purity after the reaction process. The size and appearance of beads were examined [9]. Briefly, 
an aqueous suspension of $150 \mathrm{ml}$ of beads in deionized water was placed on a chamber slide (Electron Microscopy Sciences). Bright-field images were acquired with a $5^{\prime}$ objective on an upright microscope (Zeiss Axioimager M1, Thornwood, NY) equipped with a color CCD camera (AxioVision, Zeiss).

\section{Bismuth mapping across the beads using SEM-EDX}

The beads were embedded in various mounting media, including Norland Optical Adhesive, Epoxy Resin, Polyester Resin, and Paraffin. The Norland Optical Adhesive media was used for the preparation due to its sulfur content, which aids in determining the particle edge during mapping and line scan analysis. The preparations were performed on aluminum stubs used during scanning electron microscopy. Each mount was sectioned using a microtome equipped with liquid nitrogen and glass knives. After sectioning, the preparations were evaluated for sample integrity and the prepared sections coated with -300 angstroms of gold. The visualization of the synthesized bismuth beads was carried out in a JOEL Scanning Electron Microscope, model JEM-6510 equipped with tungsten filament. Elemental analysis for bismuth was determined using EDX spectroscopy (Oxford) by using $20 \mathrm{keV}$ energy, and the line scans were generated using Aztec software. Multiple line scans through the microsphere cross-sections of the bismuth elemental map were acquired for each bead population to show the distribution of bismuth from edge to edge.

\section{Catheter Delivery Performance}

Catheter deliverability of the bismuth beads through two clinical microcatheter sizes (2.0-Fr Progreat, Terumo, Somerset, NJ and 2.4 Fr Renegade, Boston Scientific Corp.) was evaluated by administering a homogenous bead suspension in 100\% iohexol (Omnipaque 350, GE Healthcare, Waukesha, WI) as a 1:10 bead dilution and using 1 - or 3-mL syringes[10]. The catheters were laid on a bench 
with a $10 \mathrm{~cm}$ diameter coil mid-catheter to introduce curvature in the flow path. Any catheter clogging was recorded as a failure and denoted that bead size was not suitable to the respective catheter.

\section{Bismuth Beads Imaging}

The bead with the higher bismuth content and bismuth homogeneity across the bead surface was chosen for microCT and DECT evaluation.

\section{Imaging Phantom Preparation}

A suspension of bead in agarose gel ( $1 \mathrm{~mL}, 0.5 \%$ total agarose) was used to assess the radiopacity on microCT [9]. To assess radiopacity and material decomposition with DECT, iodine, bismuth beads, or a mixture of both were added to $1 \%$ agarose for a total volume of $1 \mathrm{~mL}$ For the iodine-agarose gels, four tubes were prepared using the clinical contrast agent Isovue-300 with 5, 10, 20, and $40 \mathrm{mg}$ $\mathrm{I} / \mathrm{mL}$ per tube. For the bismuth-agarose tubes, four tubes were prepared using suspensions of bismuth beads $\mathbf{6}$, with $62.5,125,250$, and $500 \mathrm{~mL}$ bismuth beads/ $\mathrm{mL}$ per tube. Similarly, agarose tubes with mixtures of Isovue-300 and bismuth beads were prepared with the following ratios of $\mathrm{mg}$ iodine $/ \mathrm{mL}$ and $\mathrm{mL}$ bismuth beads / $\mathrm{mL}: 10: 125,10: 250$, and 20:125.

\section{MicroCT}

MicroCT was performed to evaluate the radiopacity of individual bismuth beads suspended in agarose [9]. Homogeneously dispersed beads in an agarose phantom (1 mL, 0.5\% total agarose) were scanned with Bruker SkyScan 1272 (Kontich, Belgium) microCT at a nominal resolution (pixel size) of 5.0 microns with a $0.5 \mathrm{~mm}$ thick aluminum filter, a source current $125 \mathrm{~mA}$, and an applied $\mathrm{x}$-ray tube voltage of $80 \mathrm{kV}$. Reconstruction was carried out with a modified Feldkamp algorithm using the SkyScan NRecon software (Kontich, Belgium). Ring artifact 
reduction and beam hardening correction were applied. Image post-processing was performed (CT Analyser, version 1.20.3.0, Kontich, Belgium). Beads within the 70 and $150 \mathrm{~mm}$ range were selected for post-analysis, while the beads in or near a saturated spot were excluded. The voxel values were converted to HU using a water calibration phantom. A 3D mask was created for each bead to enable the calculation of $\mathrm{HU}$ per bead and analyze the variation in mean density per bead size.

\section{Dual Energy CT}

DECT imaging of the agarose gels was performed on a 192-slice DECT scanner (SOMATOM Force Dual Source, Siemens Healthcare GmbH, Forchheim, Germany). Clinical scanning protocols for the abdomen/pelvis were used with $80 \mathrm{kVp}$ imaging paired with $\mathrm{Sn} 150 \mathrm{kVp}$ and $100 \mathrm{kVp}$ paired with $\mathrm{Sn} 150 \mathrm{kVp}$, where $\mathrm{Sn} 150 \mathrm{kVp}$ refers to the use of tin filtration of the X-ray beam. Images were reconstructed at $0.5 \mathrm{~mm}$ slice thickness. The tubes were inserted in a test tube rack (Supplemental Figure 2), immersed in a water-filled container, and imaged in triplicate. Image post-processing of the data was performed with Matlab (MATLAB 2017b and Statistics Toolbox and Image Processing Toolbox, The MathWorks, Inc., Natick, MA, US).

\section{Dual Energy Index (DEI)}

Linear regression was performed between measured CT numbers versus the known iodine concentration or bismuth bead volume for both pairs of imaging exposures. The dual-energy index (DEI) was calculated from the CT numbers using the low and high energy images of each pair as follows [21, 22].

$$
D E I_{i}=\left(H U_{i}^{\text {low }}-H U_{i}^{\text {high }}\right) /\left(H U_{i}^{\text {low }}+H U_{i}^{\text {high }}+2000\right)
$$


where $H U_{i}^{\text {low }}$ and $H U_{i}^{\text {bigh }}$ are the $\mathrm{HU}$ values of material $i$ at the low energy acquisition (80 or $100 \mathrm{kVp}$ ) and the high energy acquisition $(\mathrm{Sn} 150 \mathrm{kVp}$ ), respectively. The individual attenuations are shifted by $1000 \mathrm{HU}$ to avoid negative values, which results in the factor of $2000 \mathrm{HU}$ in the denominator in the reduced equation [23]. The acquisition pair that maximizes the difference in $\mathrm{HU}$ for iodine, while minimizing the difference in $\mathrm{HU}$ for bismuth was chosen for further evaluations.

In addition, the threshold value of DEI that maximally separates the bismuth and iodine was determined, using an optimizer function (R Vienna, Austria. URL https://www.R-project.org/). A DEI image was created, and values above the threshold value were displayed as a red color map superimposed on the original DEI image. A combined map of DEI and HU values above their respective thresholds was created by blending the two maps and then superimposing the results onto the low $\mathrm{kVp}$ acquisition.

\section{Results}

\section{Synthesis}

Reactive bismuth chelating agents were successfully synthesized by alkylation or acylation of the macrocycle $\mathbf{1}$ with bifunctional linkers, as shown in Figure 5.1. These bifunctional linkers consisted of an aromatic ring with bromine or acid chloride on one end while aldehyde functional on the other end. Bead acetalation with the macrocycle was performed, and bismuth was then chelated onto the acetalated beads using $\mathrm{Bi}\left(\mathrm{CF}_{3} \mathrm{SO}_{3}\right)_{3}$ to give radiopaque beads 6 and 7 (Figure 5.1).

The acetal formation between DO3A-4-formyl benzyl (4) and the sulfonated acylamido polyvinyl alcohol beads in DMSO solvent was a second-order reaction 

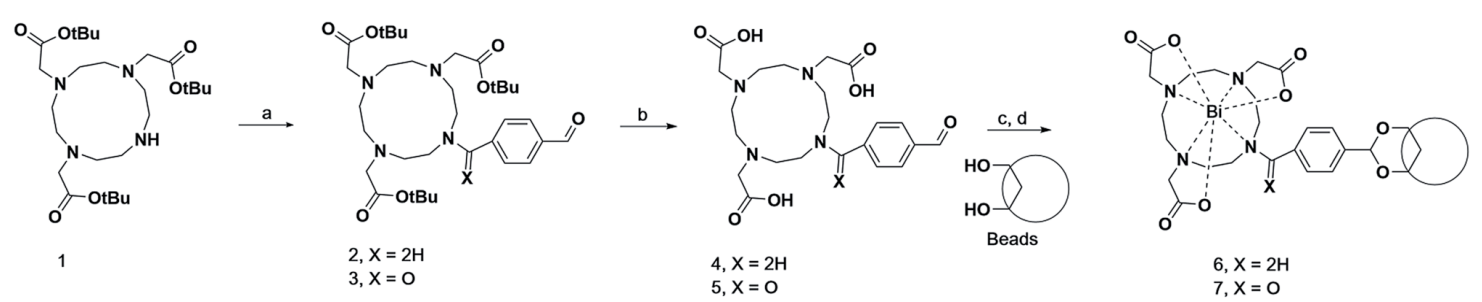

Figure 5.1 Synthesis of bismuth beads with an aromatic linker between the bead and the macrocycle. Reagent and condition: a) 4-(bromomethyl)benzaldehyde, $\mathrm{K}_{2} \mathrm{CO}_{3} / \mathrm{DMF}$ or 4-formyl benzoyl chloride, $\left.\mathrm{DCM} / \mathrm{Et}_{3} \mathrm{~N}, \mathrm{~b}\right) \mathrm{HCl} /$ dioxane, c) $\mathrm{MeSO}_{3}$ in $\mathrm{DMF}$ or $\mathrm{DMSO}, 50{ }^{\circ} \mathrm{C}$, d) $\mathrm{Bi}\left(\mathrm{CF}_{3} \mathrm{SO}_{3}\right)_{3}$, $\mathrm{pH} 8-9$ using $\mathrm{NaHCO}_{3}, 80^{\circ} \mathrm{C}$ or $\mathrm{NMP} /$ pyridine, $55^{\circ} \mathrm{C}$.

as the plot of the inverse concentration of (4) (M) vs. time (min) showed a linear relationship with reaction rate constant, $\mathrm{k}=8.5 \times 10^{-5} \mathrm{~L}_{\text {mol-1 }} \mathrm{min}^{-1}$ (Supplemental Figure 1A). Similarly, acetal formation between DO3A-4-formyl benzyl (4) and the sulfonated acylamido polyvinyl alcohol beads in DMF solvent is also second order, but the reaction rate constant was 10 -fold faster, $\mathrm{k}=8.5 \times 10^{-4} \mathrm{~L} \mathrm{mol-1} \mathrm{min}^{-1}$ (Supplemental Figure 1B).

\section{Characterization}

Optical microscopy, elemental composition, scanning electron microscopy (SEM), and energy-dispersive $\mathrm{x}$-ray analysis (EDX) were performed for both beads $\mathbf{6}$ and 7 to evaluate surface smoothness, the composition and uniformity of bismuth within and between beads.

\section{Physical Properties and Elemental Composition}

The elemental chemistry of a dry bead is $27.28 \% \mathrm{C}, 4.16 \% \mathrm{H}, 2.58 \% \mathrm{~N}, 3.3 \%$ S, 35.23\% Bi and 27.45\% O and $\mathrm{Na}$ (combined) for bead $\mathbf{6}$ and $27.85 \% \mathrm{C}$, $3.87 \% \mathrm{H}, 2.00 \% \mathrm{~N}, 3.18 \% \mathrm{~S}, 30.88 \% \mathrm{Bi}$ and $32.22 \% \mathrm{O}$ and $\mathrm{Na}$ (combined) for bead 7. The percent bismuth content from the elemental composition analysis indicated a slightly higher bismuth content for bead $\mathbf{6}$ (35.23\%) compared to bead 7 (30.88\%). Other physical characteristics of different sizes of bismuth beads are shown in Supplemental Table 1. 


\section{Optical Microscopic Evaluation}

The synthesized bismuth beads appeared dark brown and opaque under optical microscopy and maintained their spherical morphology and smoothness. However, the beads had a gross white appearance to the naked eye. The shapes and optical appearances for beads 7 are depicted in Figure 5.2.

\section{Scanning Electron Microscopy and Energy Dispersive X-ray Analysis}

SEM revealed the sectioned internal structure of both beads $\mathbf{6}$ and 7 to be free of any visible pores (Figure 5.3). Bead $\mathbf{6}$ appeared smoother than bead 7 which had an irregular margin and grain-like appearance at the bead periphery (Figure 5.3). EDX elemental map and line scans revealed higher levels and more uniformly distributed bismuth across the sectioned bead 6, compared to bead 7 (Figure 5.3). Similar to the SEM results, bead 7 demonstrated irregularity at the periphery caused by a higher density of bismuth, as can be observed in the edge intensity in the line scan as well as in the EDX elemental map (Figure 5.3). In addition, beads with higher and lower amounts of bismuth were identified. Figure $5.3 \mathrm{H}-\mathrm{K}$ shows a line scan of both a high and a low bismuth containing bead for both bead 6 and bead 7.
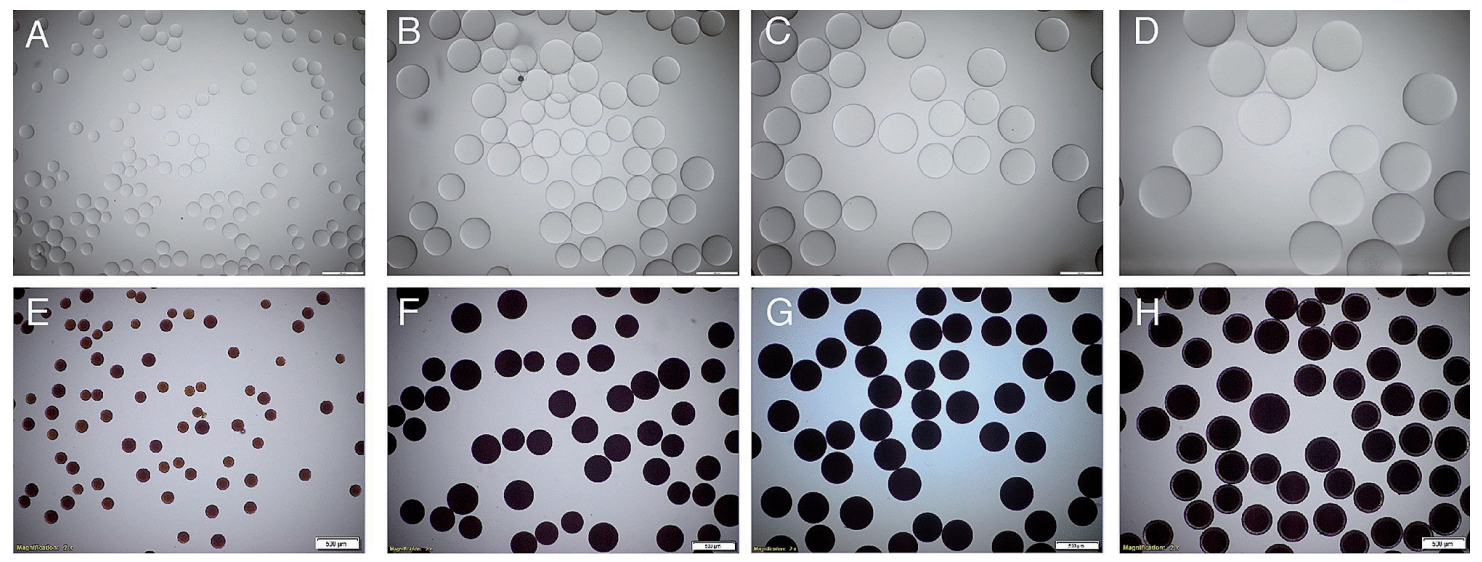

Figure 5.2 Light microscopy of beads. Size and appearance of different size of blank beads (A-D) and bismuth-functionalized beads (7) (E-H) under light microscopy, A\&E) 100-160 mm, B\&F) 250-355 mm, C\&G) 355-425 $\mathrm{mm}$ and D\&H) 425-600 mm. 

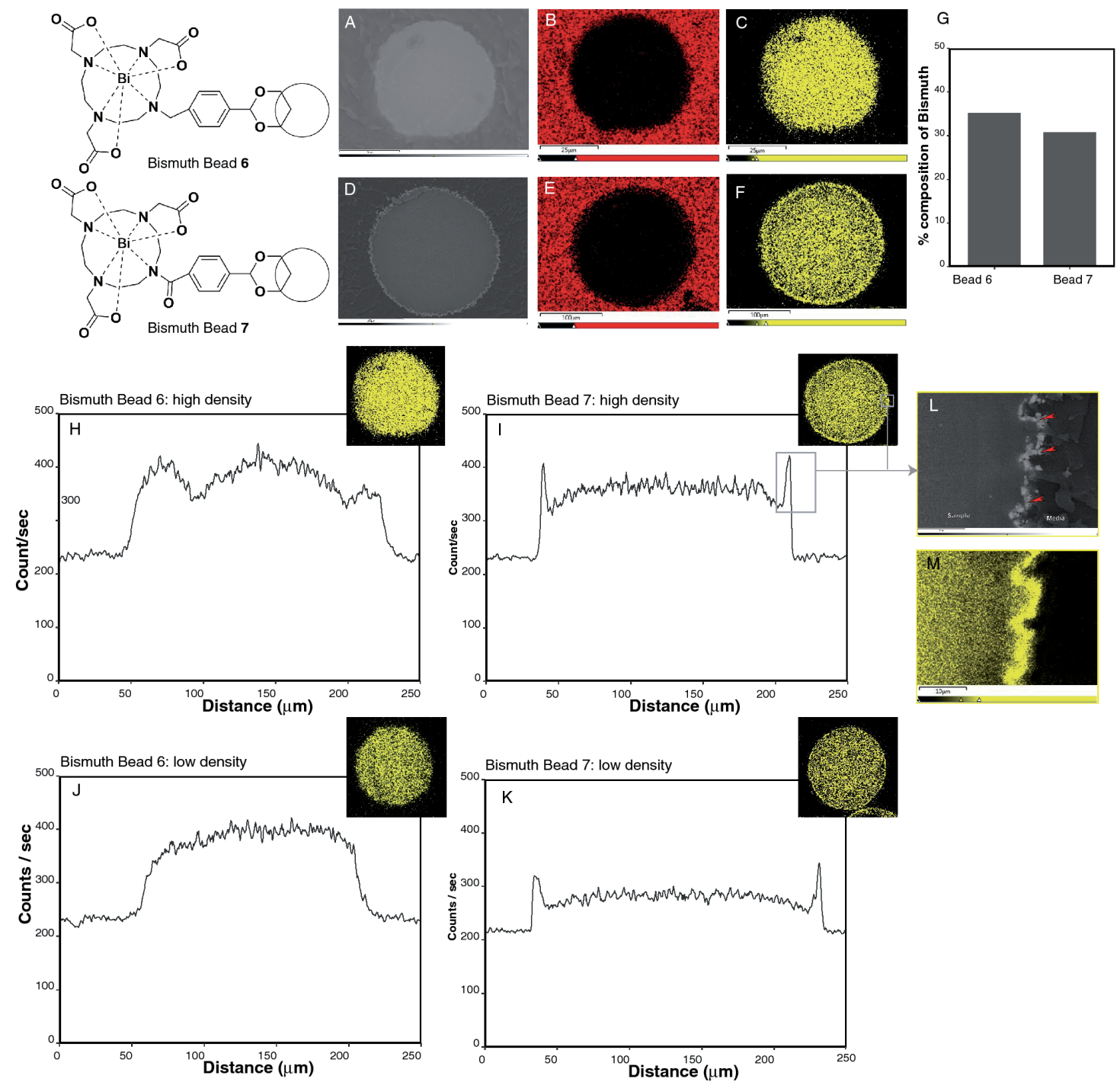

Figure 5.3 Scanning electron microscopy (SEM) and associated sulfur and bismuth elemental maps of cross-sections of bead $\mathbf{6}$ and bead 7 showing bismuth distribution. SEM image for bead $\mathbf{6}$ (A) and bead 7 (D). Associated Sulfur elemental maps for bead $\mathbf{6}(\mathbf{B})$ and bead 7 (E) and bismuth elemental maps for bead $\mathbf{6}(\mathbf{C})$ and bead $7(\mathbf{F})$. (G) Bar plot of the average percent composition of bismuth in beads 6 and 7, showing a higher average bismuth concentration for bead 6 compared to bead 7. Representative bismuth line scans of single beads with a high and low amount of bismuth, with the correlative bismuth elemental map in the upper right corner for each graph for bead $6(\mathbf{H}, \mathbf{J})$ and $7(\mathbf{I}, \mathbf{K})$. For bead 6 , the maximum bismuth count/sec was relatively constant within each bead, reflecting a homogenous distribution of bismuth throughout the beads. For bead 7, there was a peak in the bismuth counts/sec at the edge of the beads with otherwise homogeneous distribution of bismuth in the bead interior. Magnified views of the SEM (L) and bismuth element map (M) of the higher density bead 7 (I) showed an irregular bead edge and an accumulation of bismuth grains at the edge consistent with the bismuth peaks in the line graphs at both edges. Note that a slight increase in signal strength is present in the line scans between 20 and $200 \mu \mathrm{m}$. This effect is expected as the electron beam travels farther from the specimen edge. The effect is accentuated due to the small diameter of the spheres and may not indicate an actual increase in bismuth. 


\section{Microcatheter Deliverability}

Beads 7 (100-160 $\mu \mathrm{m})$ were deliverable through 2.0 Fr and 2.4 Fr catheters, while larger beads $(250-355 \mu \mathrm{m})$ required a minimum of a $2.4 \mathrm{Fr}$ catheter to enable delivery without clogging (Supplemental Table 1).

\section{Microcatheter Deliverability}

Beads $7(100-160 \mu \mathrm{m})$ were deliverable through 2.0 Fr and 2.4 Fr catheters, while larger beads $(250-355 \mu \mathrm{m})$ required a minimum of a 2.4 Fr catheter to enable delivery without clogging (Supplemental Table 1).

\section{In Vitro Evaluation with MicroCT and Clinical DECT}

Beads $\mathbf{6}$ had higher bismuth content, and bismuth was homogenously spread from edge to edge than beads 7 . Therefore, bead $\mathbf{6}$ was selected for visualization with microCT and DECT.

\section{MicroCT}

The radiopacity of bismuth bead $\mathbf{6}$ is presented in Figure 5.4. A total of 1442 bismuth beads with a size range between 70 and $150 \mathrm{~mm}$ were selected for post-analysis after exclusion of beads near saturation on the image density scale, representing $1.4 \%$ of the original number. The mean Hounsfield units (HU) for the entire population of beads was $5200 \pm 846 \mathrm{HU}$. There was no correlation between the bead volume and HU (Figure 5.4); however, a histogram of the beads revealed two distinct HU intensity peaks. Dividing the beads into two groups with a $4800 \mathrm{HU}$ threshold revealed that the lower density bead population had a mean HU of $3972 \pm 365$ (71\% of the bead), while the higher density bead population had a mean HU of $5700 \pm 325$ (29\% of the beads). 

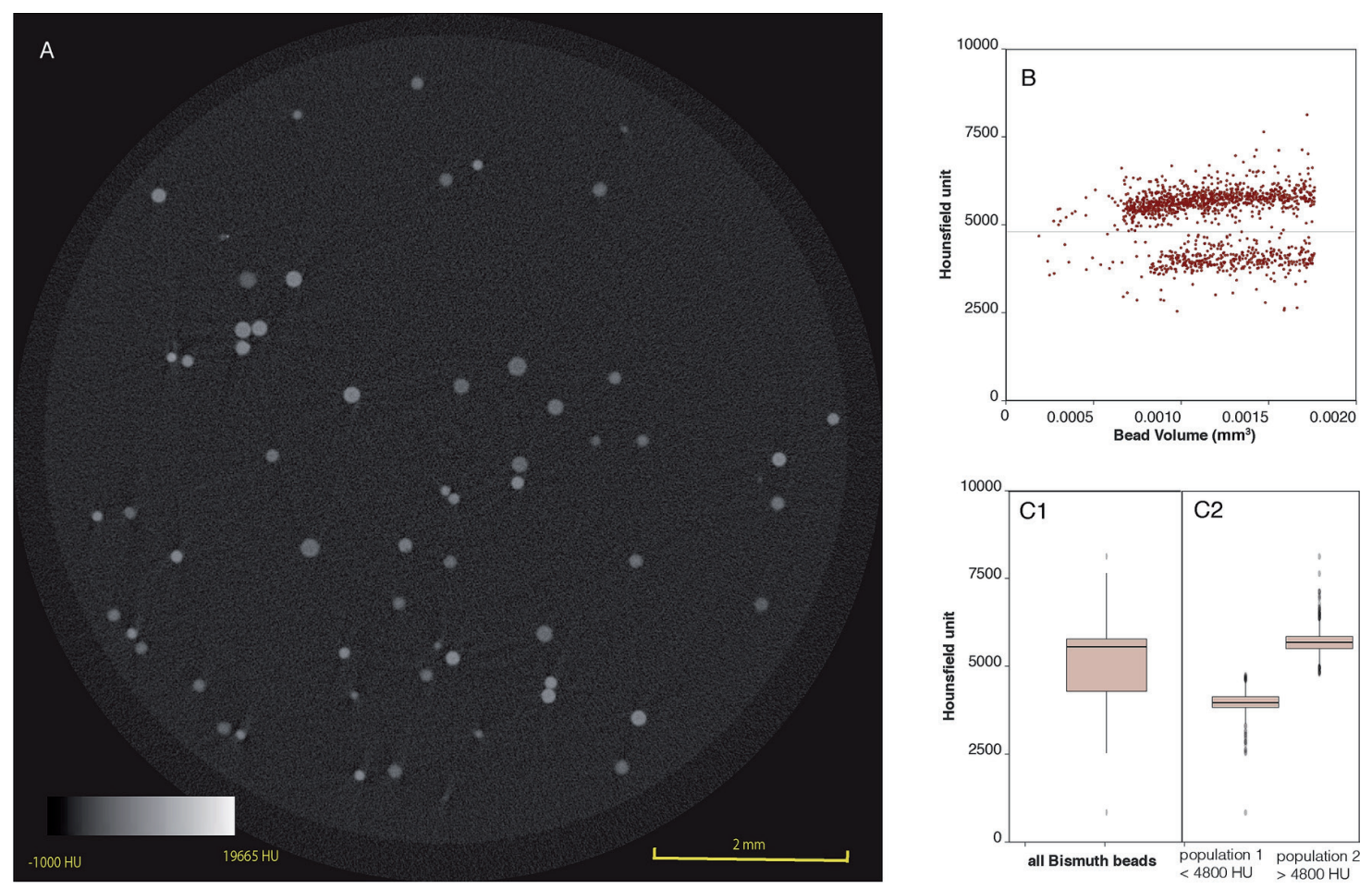

Figure 5.4 MicroCT imaging of bead 6. A) Axial slice of a suspension of bead 6. B) Scatterplot revealing two bead subpopulations that can be distinguished at a 4800 threshold. There was no correlation between bead volume and HU. C) Boxplots of HU for all bismuth beads ranging 70-150 mm (C1) and both bead populations (C2) revealing the average intensity for both bead populations.

\section{Dual Energy CT}

There was a linear correlation between CT number and both iodine concentration and bismuth bead volume/mL at each energy level (Figure 5.5) of microsphere 6 . For iodine, the largest difference in $\mathrm{HU}$ value was observed between $80 \mathrm{kVp}$ and Sn $150 \mathrm{kVp}$ acquisitions, as compared to the 100 and Sn $150 \mathrm{kVp}$. For bismuth, the HU difference was slightly more between 100 and Sn150 kVp; however, both acquisitions would be feasible to image bismuth with minimum HU differences.

The final material decomposition analysis was performed using the $80 \mathrm{kVp}$ and $\mathrm{Sn} 150 \mathrm{kV}$ p paired acquisition, as the iodine extraction was favorable for this acquisition pair. There was a good linear correlation between the iodine 

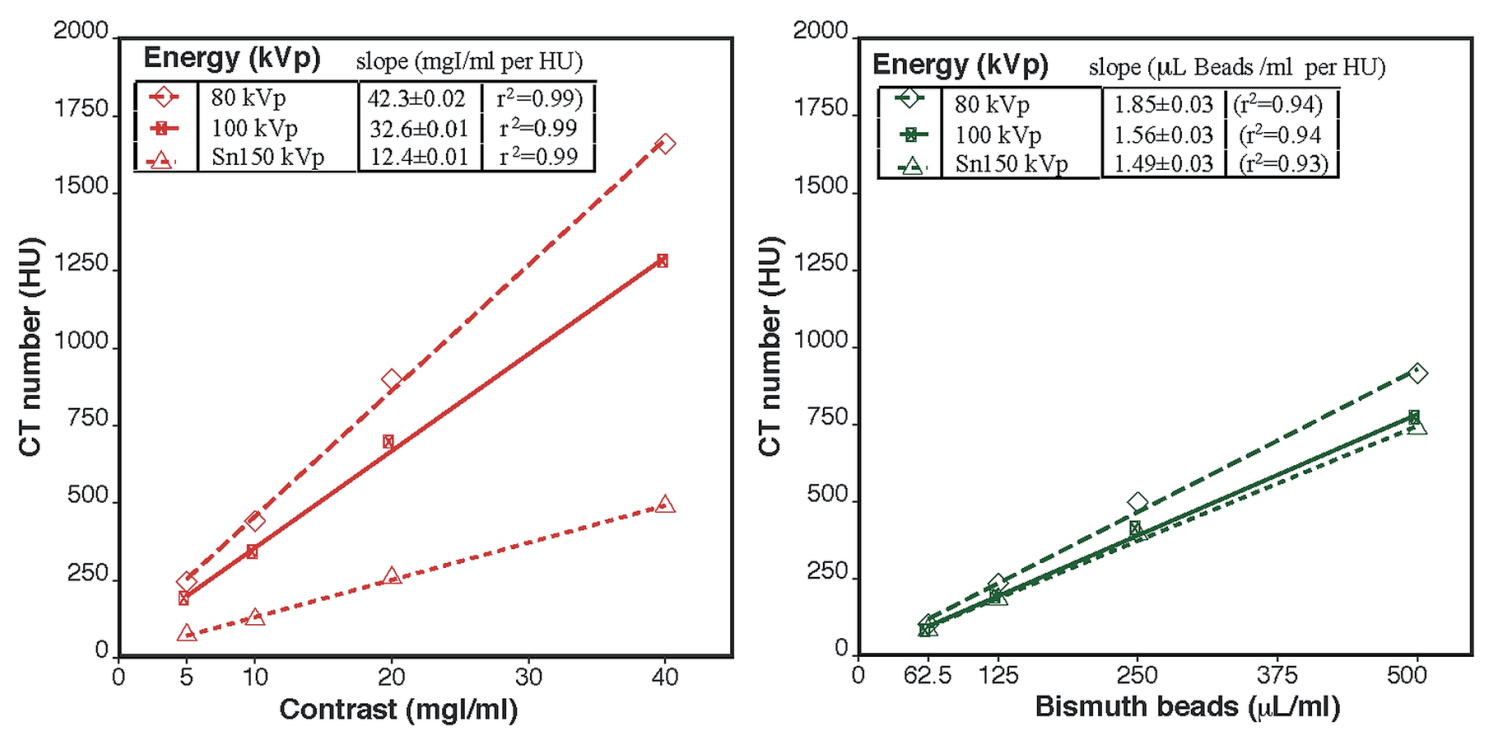

Figure 5.5 Clinical dual energy CT imaging. Linear regression between the image intensity or CT number (HU) for each of three energy levels and agarose gel content, either iodine (left) or bismuth beads 6 (right).

concentration and dual energy index (DEI) $\left(\mathrm{r}^{2}=0.9, \mathrm{p}<0.01\right)$, while there was a poor linear correlation between the bismuth volume and DEI $\left(\mathrm{r}^{2}=0.34, \mathrm{p}<\right.$ 0.01) as shown in Figure 5.6. The DEI threshold that optimally separated iodine and bismuth was defined at $0.06 .99 .9 \%$ of the voxels in the $10 \mu \mathrm{L} \mathrm{I} / \mathrm{mL}$ tubes were above this threshold, while $99.7 \%$ of the $62.5 \mathrm{~mL} / \mathrm{mL}$ voxels in the bismuth agarose solution were excluded (Figure 5.7). For the mixed tubes, the HU of all voxels were above the threshold (Supplemental Table 2).

The DEI data derived from the $80 \mathrm{kVp}$ and $\mathrm{Sn} 150 \mathrm{kVp}$ images and subjected to the 0.06 DEI threshold confirms that the imaging identifies tubes that contain iodine, whether alone or in combination with bismuth beads (Figure 5.8). The $\mathrm{HU}$ map with the $20 \mathrm{HU}$ threshold to exclude the agarose identified both iodine and bismuth within the tubes. The combination of the two maps (DEI map to identify iodine and HU to identify any radioabsorber) demonstrates that bismuth alone could be distinguished from iodine alone or the combination of iodine and bismuth. 

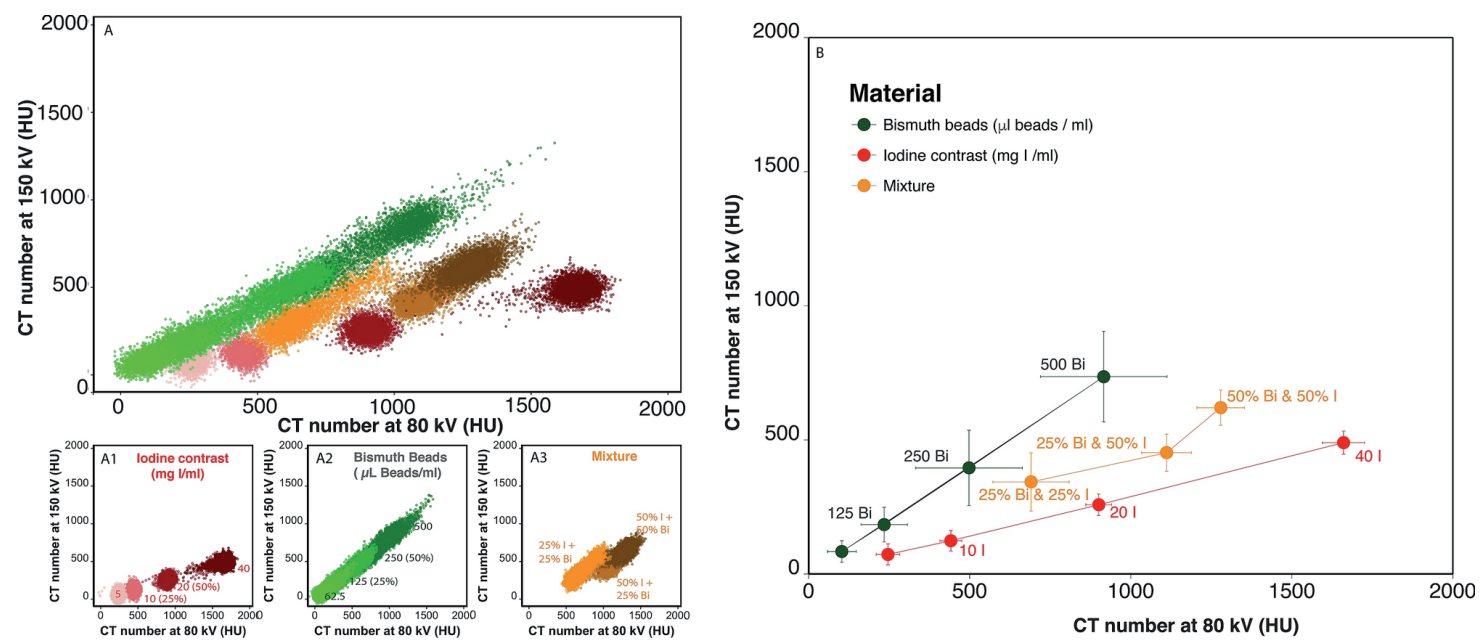

Figure 5.6 Correlation of CT numbers (HU) on dual energy CT at $80 \mathrm{kVp}$ vs $\mathrm{Sn} 150 \mathrm{kVp}$ : iodine, bismuth beads (6) and mixture of both. A) Scatter plots of the CT numbers (HU) derived from the $80 \mathrm{kVp}$ and $\mathrm{Sn} 150 \mathrm{kVp}$ scans for each voxel. Subplots are shown as A1) iodine (red), A2) bismuth (green), and A3) a mixture of both (orange). B) The average and standard deviation (bars) of the CT number (HU) derived from the $80 \mathrm{kVp}$ and $\mathrm{Sn} 150 \mathrm{kVp}$ scans for each tube.
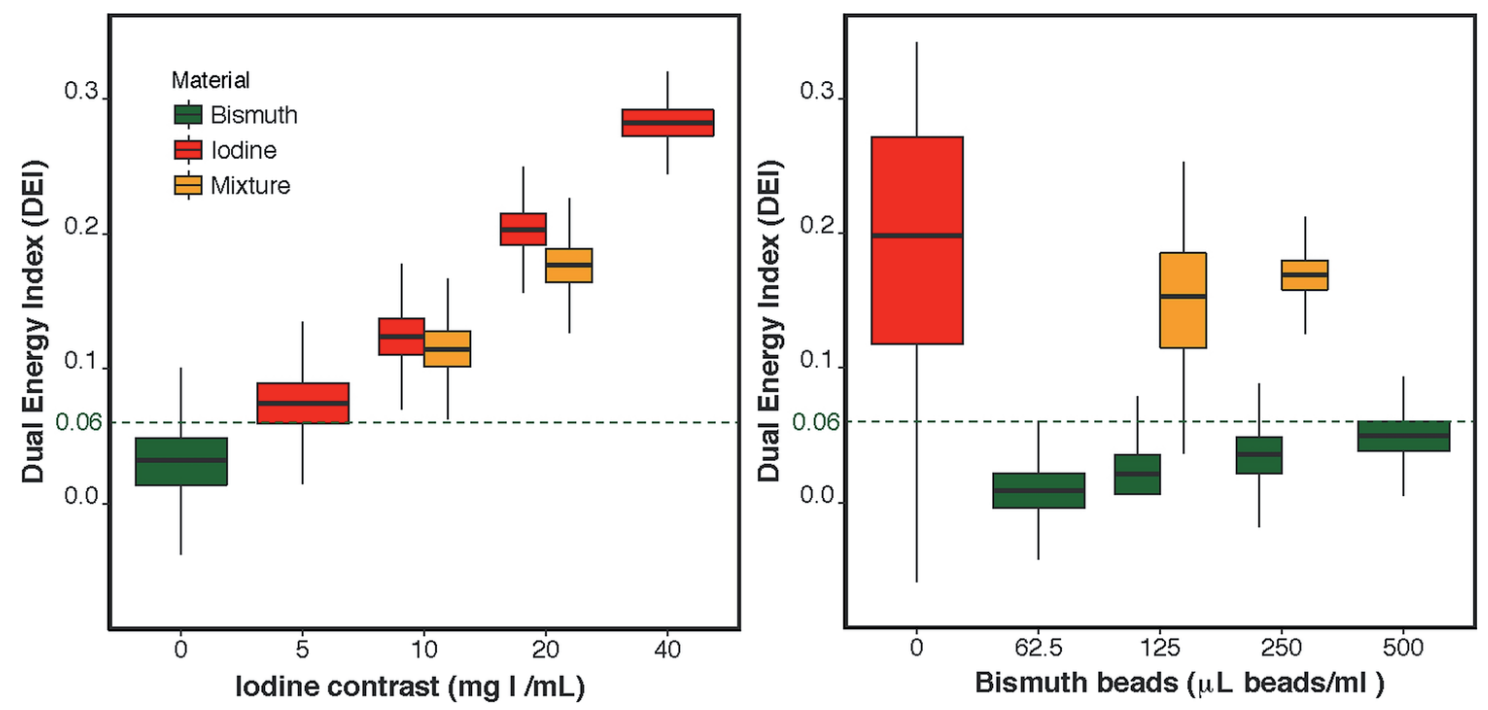

Figure 5.7 Boxplot of the dual energy index (DEI) calculated per voxel averaged per iodine concentration (left) or bismuth beads (right). The green line represents the DEI threshold of 0.06 which optimally classified iodine. 


\section{$80 \mathrm{kVp}$ original}

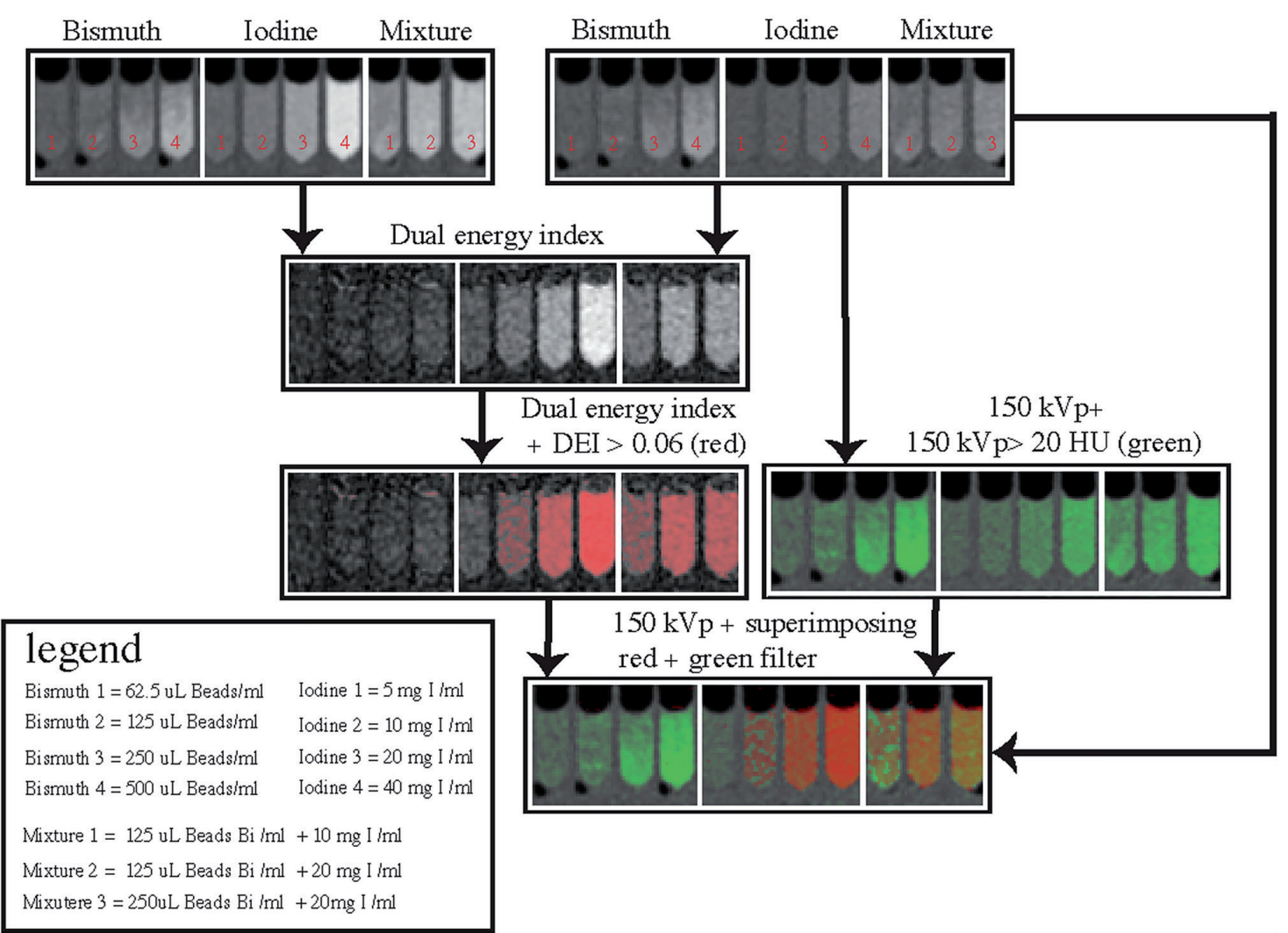

Figure 5.8 Dual energy CT post-processing workflow and outcome for bismuth, iodine and mixture agarose phantoms. Row 1: Original $80 \mathrm{kVp}$ and $\mathrm{Sn} 150 \mathrm{kVp}$ energy reconstructions. Row 2: A dual energy index (DEI) image is calculated from the two scans. Row 3: DEI values greater than 0.06 are superimposed on the original DEI image as a red color map. (left image). Image intensity values above $20 \mathrm{HU}$ in the $\mathrm{Sn} 150 \mathrm{kVp}$ image are superimposed on the original image as a green color map (right image). Row 4: Combined DEI and HU color maps as blended color maps superimposed on the $\mathrm{Sn} 150 \mathrm{kVp}$ image. High HU above the threshold (green) occurs for both iodine and bismuth, however, the increase in DEI (red) only occurs for iodine. Therefore, bismuth alone can be differentiated from either iodine alone or a bismuth/iodine mixture, due to the absence of red. 


\section{Discussion}

In this study, two types of bismuth beads were synthesized having very similar linkers but with two different bismuth chelation techniques. For bismuth bead $\mathbf{6}$, chelation was performed in aqueous media at $\mathrm{pH} 8-9$, and for bismuth bead 7 an organic solvent was chosen. It was shown with elemental composition and SEMEDX experiments that bead $\mathbf{6}$ had a higher bismuth content and a more uniform distribution across the entire bead surface compared to bead 7. Therefore, bead $\mathbf{6}$ was chosen for subsequent characterization and perhaps for future in vivo works. MicroCT of bead $\mathbf{6}$ showed that there were two populations of bead $\mathbf{6}$ with higher and lower radiographic density. Even though these two bead populations had different bismuth content, bismuth was relatively homogenous across the beads on SEM-EDX analysis. Both bead $\mathbf{6}$ subpopulations had a sufficiently high HU per bead to be clinically useful, with means of $3972 \mathrm{HU}$ and $5700 \mathrm{HU}$. A plausible explanation for having two sets of beads with different bismuth contents might be related to variations in the number of macrocycles acetalated to the various beads, the amount of bismuth chelated to the macrocycle containing bead, or both. The overall radiopacity and standard deviation of the bismuth beads is in the same range as the radiopacity of the clinically utilized iodine-based radiopaque beads $4718 \pm 257 \mathrm{HU}[2]$.

For clinical delivery, beads should have a stable suspension in liquid contrast and must be able to pass through a microcatheter[10]. The bismuth beads in the range of 100-160 mm were deliverable through both 2.0 and $2.4 \mathrm{Fr}$ clinical microcatheters, while the larger beads $(250-355 \mathrm{~mm})$ required a $2.4 \mathrm{Fr}$ microcatheter. Both beads sizes are comparable to those in standard clinical use. 
DECT imaging may be used for material classification by exploiting the differences in X-ray absorption of iodine and bismuth from high radiographic density. This model could also be imposed in the clinical setting of liver tumor embolization. A DEI threshold of 0.06 distinguished iodine from the background and from bismuth beads alone. However, there was an overlap in DEI values for the higher concentrations of bismuth beads and the lower concentrations of iodine. Therefore, the DEI as a single image feature could be used to identify if iodine was present, either alone or in combination with bismuth beads. The model dictates that densities (HU) above

background must be either iodine or bismuth, while any tube with a DEI below the threshold that does not contain iodine and must be bismuth beads. For embolization, the only density would be iodine or bismuth beads. Refining and optimizing the synthesis process to increase the selection of only the bead populations with higher radiographic density could lead to an increase in the signal to noise ratio in CT scanning and material decomposition. The DEI could be used to define and follow the washout of iodine contrast after embolization. Post-embolization residual tumor enhancement may be hard to distinguish from high attenuation iodine beads, without a subtraction image in conventional CT. Bismuth beads would not present this problem[24]. These novel bismuth beads have the potential to improve image-guided local drug delivery in patients with liver cancer by directly identifying either non-targeted delivery or undertreated tumor. The ability to choose from two bead formulations to load drugs opens the door to dual drug delivery and "dual drug dose tracking". Two beads could be loaded with doxorubicin and a second agent, such as an immunomodulator. Two drugs could be selected and targeted with a mechanistic rationale that factors in the tumor microenvironment or molecular data. For example, doxorubicin intercalates in the nucleus and is more active for cell-cycle-specific regions of high 
mitosis, which may be reflected by high metabolic activity on FDG PET. Necrosistargeting agents could be loaded and localized by injection into imaged regions of necrosis or high interstitial pressure, such as regions with restricted diffusion on MRI. Anti-vascular agents loaded on an image-able bead could be targeted to areas of high enhancement or vascular density, also easily localized on imaging. Fused imaging on top of procedural CBCT also allows exquisite mechanistic targeting, in such a paradigm[25]. Combining iodine-based and a bismuth-based beads loaded with different chemotherapy or immunotherapy drugs, could potentially enable dual drug delivery for a more rational, fusion image guided therapy grounded in mechanistic molecular reasoning. Such a paradigm could move image guided therapies into a new era after the field has delivered the same anthracycline for over four decades[26].

The development of more advanced material decomposition-based algorithms, artificial intelligence-based CT image reconstruction, or artificial intelligencebased segmentation may further enable iodine/bismuth material classification and drug dose maps for treatment plans. Higher-resolution imaging and advanced material decomposition may be further explored in the next-generation CT scanners; photon counting CT, for example, can distinguish between bismuth, gadolinium, and iodine, in vivo[27].

\section{Limitations and Future Work}

Drug loading and elution profiles of the derived bismuth beads were not evaluated in this study. Based on the chemistry of the bismuth beads, it is expected that elution profiles will parallel its predecessor iodine-based radiopaque beads' loading and elution profiles, but additional work will be required to confirm. The primary goal of the current study was to synthesize and characterize a radiopaque bismuth 
bead. In vitro evaluation of small agarose volumes containing beads may not reflect the full signal-to-noise levels observed in vivo with motion and in vivo environmental factors. In vivo preclinical studies were beyond the scope of this paper but are required to further explore the radiopacity and performance of the beads. However, the radiopacity of the beads on DECT was well above that of normal liver, so beads packed within hepatic arteries will be visible within the liver. While the bismuth is expected to remain bound to the chelation macrocycle [28, 29], in vivo safety studies are required, which may also include an assessment of the embolization or drug-eluting effect of the beads in tissues.

\section{Conclusion}

In this study, we present facile methods for the synthesis of radiopaque polyvinyl alcohol-based microspheres, or beads, based on a marketed microsphere subsequently modified with a bifunctional macrocycle and chelated with bismuth. Bismuth distribution was uniform across the bead $\mathbf{6}$. The radiopacity of the bismuth beads was confirmed by microCT and DECT. Furthermore, DECT imaging permitted material classification (decomposition) that could distinguish bismuth beads alone from iodinated contrast or a mixture of iodine and bismuth beads, which should also extend to discrimination between iodinated beads and bismuth beads. Imaging of two distinct bead populations may enable and promote the development of dual drug delivery and dual drug tracking when the drugs are delivered by beads that can be distinguished on imaging. The combination of drugs, devices, and imaging-guidance with dual drug mapping is enabled by dual bead localization, which could stimulate the field to evolve beyond a four decades history of using the same drug, doxorubicin, delivered via similar catheters with similar x-ray guidance. 


\section{Acknowledgments}

This work was supported by the Center for Interventional Oncology in the Intramural Research Program of the National Institutes of Health (NIH) by intramural NIH Grants NIH Z01 1ZID BC011242 and CL040015. The NIH and Biocompatibles UK Ltd (BTG/Boston Scientific) have a Cooperative Research and Development Agreement, which provided funding for the conduct of the study. NIH and Biocompatibles UK Ltd have intellectual property in the field. Thanks to Dr. Martin W. Brechbiel, Radioimmune \& Inorganic chemistry Section, Radiation Oncology Branch, NCI, Drs. John Lloyd and Noel Whittaker, NIDDK Advanced Mass Spec Core Facility for recording mass spectroscopy data, and Dr. Belhu Metaferia, NIDDK, National Institutes of Health, for fruitful discussion. Thanks to Jayasai Rajagopal for constructive discussion on the DECT imaging, and to Amir Pourmorteza, David Bluemke, and Elizabeth Jones for discussion of photon-counting CT and Kevin Tixier for his help on taking images of beads with an upright microscope. 


\section{References:}

1. Heimbach, J.K., et al., AASLD guidelines for the treatment of hepatocellular carcinoma. Hepatology, 2018. 67(1): p. 358-380.

2. Ashrafi, K., et al., Characterization of a novel intrinsically radiopaque drug-eluting bead for image-guided therapy: DC Bead LUMI ${ }^{\mathrm{TM}}$. J. Control. Release, 2017. 250: p. 36-47.

3. Negussie, A.H., et al., Synthesis and characterization of image-able polyvinyl alcohol microspheres for image-guided chemoembolization. J. Mater. Sci.Mater. Med., 2015. 26(6).

4. Hasan, M.S., S. Kehoe, and D. Boyd, Temporal analysis of dissolution by-products and genotoxic potential of spherical zinc-silicate bioglass: "Imageable beads" for transarterial embolization. J. Biomater. Appl., 2014. 29(4): p. 566-581.

5. Thanoo, B.C. and A. Jayakrishnan, Barium sulfate loaded $p$ (HEMA) microspheres as artificial emboli-preparation and properties. Biomaterials, 1990. 11(7): p. 477-481.

6. Zeng, J., et al., Radiopaque and uniform alginate microspheres loaded with tantalum nanoparticles for real-time imaging during transcatheter arterial embolization. Theranostics, 2018. 8(17): p. 4591-4600.

7. Lewis, A.L. and M.R. Dreher, Locoregional drug delivery using image-guided intra-arterial drug eluting bead therapy. J. Control. Release, 2012. 161(2): p. 338-350.

8. Lewis, A.L., et al., Bench-to-clinic development of imageable drug-eluting embolization beads: finding the balance. Future Oncol, 2018. 14(26): p. 2741-2760.

9. Johnson, C.G., et al., Preparation of radiopaque drug-eluting beads for transcatheter chemoembolization. J. Vasc. Interv. Radiol., 2016. 27(1): p. 117-126.

10. Duran, R., et al., A Novel inherently radiopaque bead for transarterial embolization to treat liver cancer-a pre-clinical study. Theranostics, 2016. 6(1): p. 28-39.

11. Aliberti, C., et al., Transarterial chemoembolization with DC Bead LUMI radiopaque beads for primary liver cancer treatment: preliminary experience. Future Oncol, 2017. 13(25): p. 2243-2252.

12. Levy, E.B., et al., First human experience with directly image-able iodinated embolization microbeads. Cardiovas. Interv. Radiol., 2016. 39(8): p. 1177-1186.

13. Mikhail, A.S., et al., Mapping drug dose distribution on CT images following transarterial chemoembolization with radiopaque drug-eluting beads in a rabbit tumor model. Radiology, 2018. 289(2): p. 396-404.

14. Mikhail, A.S., et. al., Contrast clearance following hepatic transarterial embolization with radio-opaque and non-radio-opaque micro beads in swine. 2018; Available from: https://cslide. ctimeetingtech.com/cirse2018/attendee/person/145341.

15. McCollough, C.H., et al., dual and multi-energy CT: principles, technical approaches, and clinical applications. Radiology, 2015. 276(3): p. 637-653.

16. Mondal, R. and A.K. Mallik, Recent applications of potassium carbonate in organic synthesis. Org. Prep. Proced. Int., 2014. 46(5): p. 391-434.

17. Iserloh, U., et al., Synthesis of $(R, R)-4,6$-dibenzofurandiyl-2,2'-bis (4-phenyloxazoline) (DBFOX/PH)-a novel tridentate ligand. Org. Synth., 2003. 80: p. 46-56.

18. Bhakuni, B.S., et al., Potassium tert-butoxide mediated synthesis of phenanthridinone. Org. Synth., 2013. 90: p. 164-173.

19. Muhlemann, C., P. Hartmann, and J.-P. Obrecht, Erratum for: Titanium-mediated addition of silyl dienol ethers to electrophilic glycine: 4-ketopipecolic acid hydrochloride1. Org. Synth., 2016. 93: p. 37-48. 
20. Johar, G.S., New spot tests for thallium(I), bismuth (III), and iodine. Mikrochimi. Acta, 1975(1): p. 19-23.

21. Johnson, T.R.C., et al., Material differentiation by dual energy CT: initial experience. Eur. Radiol., 2007. 17(6): p. 1510-1517.

22. Spek, A., et al., Dual energy can accurately differentiate uric acid-containing urinary calculi from calcium stones. World J. Urolo., 2016. 34(9): p. 1297-1302.

23. Barreto, M., et al., Potential of dual-energy computed tomography to characterize atherosclerotic plaque: ex vivo assessment of human coronary arteries in comparison to histology. J. Cardiovasc. Comput. Tomogr., 2008. 2(4): p. 234-42.

24. Sharma, K.V., et al., Long-term biocompatibility, imaging appearance and tissue effects associated with delivery of a novel radiopaque embolization bead for image-guided therapy. Biomaterials, 2016. 103: p. 293-304.

25. Abi-Jaoudeh, N., et al., Image fusion during vascular and nonvascular image-guided procedures. Tech. Vasc. Interv. Radiol., 2013. 16(3): p. 168-76.

26. Guan, Y.-S., Q. He, and M.-Q. Wang, Transcatheter arterial chemoembolization: history for more than 30 years. ISRN Gastroenterol, 2012. 2012: p. 480650.

27. Symons, R., et al., Photon-counting CT for simultaneous imaging of multiple contrast agents in the abdomen: an in vivo study. Med. Phys., 2017. 44(10): p. 5120-5127.

28. Tubafard, S. and S.J. Fatemi, Chelation of bismuth by combining desferrioxamine and deferiprone in rats. Toxicol. Ind. Health, 2008. 24(4): p. 235-240.

29. Wilson, J.J., et al., Evaluation of nitrogen-rich macrocyclic ligands for the chelation of therapeutic bismuth radioisotopes. Nucl. Med. Biol., 2015. 42(5): p. 428-438. 


\section{Supplemental Information}

\section{Determination of solid content and bismuth concentration of hydrated}

\section{beads}

To calculate the equilibrium water content and solid content, between 0.2 and $0.3 \mathrm{~mL}$ of sedimented beads (bead 7) were washed 3 times with $10 \mathrm{~mL}$ of deionized water and placed on a shaker plate at $250 \mathrm{rpm}$ for 5 minutes in order to remove residual salts present in the packing solution. The washed beads were then transferred to a pre-weighed aluminum plate and a minimum amount of deionized water to aid the complete transfer of beads. Excess water was removed using a Pasteur pipette followed by pressing a filter paper gently against the beads. The aluminum plates containing beads were then transferred to a vacuum oven and the samples were allowed to dry at a pressure of 50-100 mbar and $50{ }^{\circ} \mathrm{C}$. After 12 hours, the aluminum plates containing the dried beads were weighed to calculate equilibrium water content.

\section{Catheter Delivery Performance}

Catheter deliverability of the bismuth beads through two clinical microcatheter sizes (2.0-Fr Progreat, Terumo, Somerset, NJ and 2.4 Fr Renegade, Boston Scientific Corp.) was evaluated by administering a homogenous bead suspension in 100\% iohexol (Omnipaque 350, GE Healthcare, Waukesha, WI) as a 1:10 bead dilution and using 1- or 3-mL syringes, mixed with Omnipaque 350, through two clinical microcatheter sizes $(2.0 \mathrm{~F}$ and $2.4 \mathrm{~F})$ was evaluated 10 . The deliverability of the beads, mixed with iohexol contrast (Omnipaque 350, GE Healthcare, Waukesha, WI ), through two clinical microcatheter sizes $(2.0 \mathrm{~F}$ and $2.4 \mathrm{~F})$ was evaluated as reported previously 10. Catheter deliverability was determined by administering a homogenous bead suspension in $100 \%$ iohexol $(1: 10$ bead dilution, 1 - or 3-mL syringe). The catheters were laid on a bench with a $10 \mathrm{~cm}$ 
diameter coil mid-, and at the center of the catheter a coil $(10 \mathrm{~cm}$ diameter $)$ was introduced to produce a more tortuous to introduce curvature in the flow path for the beads to navigate thorough (a curvature catheters may experience in a patient). Any catheter clogging was recorded as a failure and denoted that bead size was not suitable to the respective catheter. Catheters included in this study are 2.0-Fr Progreat (Terumo, Somerset, NJ) and 2.4 Fr Renegade (Boston Scientific Corp.).
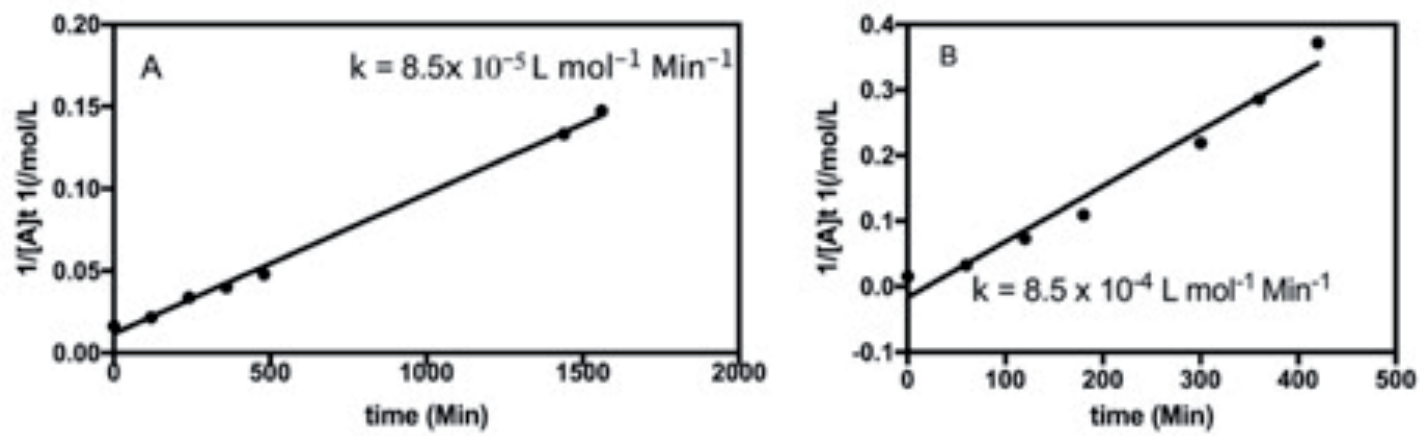

Supplemental Figure 1. Acetalation reaction of the beads with 4 in A) DMSO and B) in DMF with second-order reaction rate with rate constant $\mathrm{k}=8.5 \times 10^{-5} \mathrm{~L} \mathrm{~mol}^{-1} \mathrm{Min}^{-1}$ and $\mathrm{k}=8.5 \times 10^{-4} \mathrm{~L}$ $\mathrm{mol}^{-1} \mathrm{Min}^{-1}$, respectively. Where $[\mathrm{A}] \mathrm{t}$ is concentration of 4-(bromomethyl)benzaldehyde over time. 
Supplemental Table 1. Physical characterization of different sizes of bismuth beads (radiopaque bead 7)

\begin{tabular}{|c|c|c|c|c|}
\hline \multirow[t]{2}{*}{ Properties } & \multicolumn{4}{|c|}{ Bismuth bead size $(\mu \mathrm{m})$} \\
\hline & $100-160$ & $250-355$ & $355-425$ & $425-600$ \\
\hline \multicolumn{5}{|l|}{ Physical characterization } \\
\hline $\begin{array}{l}\text { Solid content of hydrated bead } \\
(\mathrm{mg} / \mathrm{mL})\end{array}$ & 195.4 & 190.9 & 157.1 & 169.7 \\
\hline $\begin{array}{l}\text { Bismuth concentration }(\mathrm{mg} / \mathrm{mL}) \\
\text { hydrated bead }\end{array}$ & 69.3 & 49.5 & 48.6 & 51.2 \\
\hline \multicolumn{5}{|l|}{ Delivery performance } \\
\hline $\begin{array}{l}\text { Deliverability }(2.0 \mathrm{Fr})-(\text { hydrated } \\
\text { beads diluted in contrast })\end{array}$ & Yes & Yes-moderate & No & No \\
\hline $\begin{array}{l}\text { Deliverability ( } 2.4 \mathrm{Fr}) \text { (hydrated } \\
\text { beads diluted in contrast) }\end{array}$ & Yes & Yes & Yes-moderate & No \\
\hline
\end{tabular}

Supplemental Table 2. Mean and SD of the Hounds field voxel values for each tube in $80,150 \mathrm{kv}$ and the DEI.

\begin{tabular}{|c|c|c|c|c|c|c|}
\hline Tube & $\begin{array}{l}\text { Iodine } \\
\text { Component }\end{array}$ & $\begin{array}{l}\text { Bismuth } \\
\text { Component }\end{array}$ & $\begin{array}{l}80 \mathrm{kV} \mathrm{HU} \\
\text { mean }(\mathrm{SD})\end{array}$ & $\begin{array}{l}150 \mathrm{kV} \mathrm{HU} \\
\text { mean }(\mathrm{SD})\end{array}$ & $\begin{array}{l}\text { DEI } \\
\text { mean }(S D)\end{array}$ & $\begin{array}{l}\text { DEI }>0.06 \\
(\%)\end{array}$ \\
\hline \multicolumn{7}{|c|}{ Iodine Contrast $(\mathrm{mgI} / \mathrm{mL})$} \\
\hline 5 & $12.50 \%$ & $0 \%$ & $246(37)$ & $73(40)$ & $0.07(0.02)$ & $74.3 \%$ \\
\hline 10 & $25 \%$ & $0 \%$ & $442(34)$ & $124(38)$ & $0.12(0.02)$ & $99.9 \%$ \\
\hline 20 & $50 \%$ & $0 \%$ & $901(40)$ & $258(40)$ & $0.20(0.02)$ & $100.0 \%$ \\
\hline 40 & $100 \%$ & $0 \%$ & $1660(65)$ & $490(43)$ & $0.28(0.02)$ & $100.0 \%$ \\
\hline \multicolumn{7}{|c|}{ Bismuth beads ( $\mu \mathrm{L}$ beads $/ \mathrm{mL})$} \\
\hline 62.5 & $0 \%$ & $12.50 \%$ & $103(45)$ & $84(40)$ & $0.01(0.02)$ & $0.3 \%$ \\
\hline 125 & $0 \%$ & $25 \%$ & $235(72)$ & $184(65)$ & $0.02(0.02)$ & $3.4 \%$ \\
\hline 250 & $0 \%$ & $50 \%$ & $496(166)$ & $395(140)$ & $0.03(0.02)$ & $9.6 \%$ \\
\hline 500 & $0 \%$ & $100 \%$ & $916(196)$ & $736(169)$ & $0.05(0.02)$ & $25.8 \%$ \\
\hline \multicolumn{7}{|c|}{ Mixed $(\mu \mathrm{L}$ beads $/ \mathrm{mL}+(\mathrm{mgI} \mathrm{I} / \mathrm{mL})$} \\
\hline $125 \mathrm{Bi}+10 \mathrm{I}$ & $25 \%$ & $25 \%$ & 690(119) & $343(109)$ & $\begin{array}{c}0.11 \\
(0.02)\end{array}$ & $99.7 \%$ \\
\hline $125 \mathrm{Bi}+20 \mathrm{I}$ & $50 \%$ & $25 \%$ & $1111(77)$ & $452(69)$ & $0.19(0.02)$ & $100.0 \%$ \\
\hline $250 \mathrm{Bi}+20 \mathrm{I}$ & $50 \%$ & $50 \%$ & $1279(74)$ & $620(66)$ & $0.17(0.02)$ & $100.0 \%$ \\
\hline
\end{tabular}




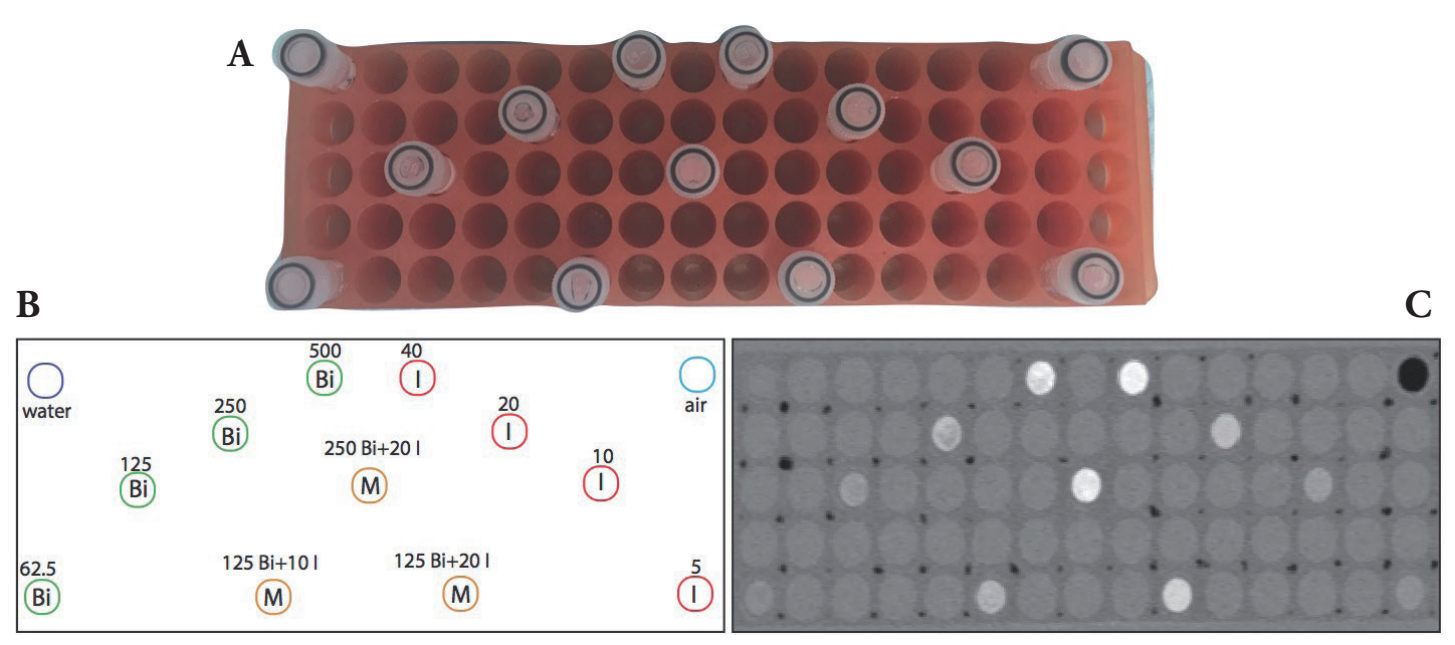

Supplemental Figure 2. DECT of agarose tubes with iodine contrast ( $\mathrm{mg} \mathrm{I} / \mathrm{mL}$, red circles), bismuth beads ( $\mathrm{mL}$ beads $/ \mathrm{mL}$, green circles) or a mixture (orange circle). A) Arrangement of tubes in the rack. B) Tube contents and concentrations in agarose. C) Axial CT acquisition from the $\mathrm{Sn} 150 \mathrm{kVp}$ scan. 


\section{Chapter 6}

Summarizing discussion 
The three main cancer treatment modalities are surgery, radiotherapy, and chemotherapy [1-6]. Unfortunately, they are too often associated with high risks of injury, or toxicity to healthy tissues. Therefore, more selective treatment paradigms and adjuvant combination methods that can kill tumor cells with the promise of limited or no side-effects are being investigated including immunotherapy, drug delivery systems (DDS), and drug delivery devices (DDDs). Ideally, combining two or more of these strategies will realize even more selective treatments with better safety and efficacy. One such novel approach involves the combination of DDSs with minimally or noninvasive heat deploying devices that aid drug release from the DDSs or increase the localization of DDSs in malignant tissues, to maximize the drug's therapeutic index, i.e., widening the therapeutic window for drugs that are effective otherwise in 'free' form but then exert toxicities at nearly the same dose ranges. This combination of drug and device aims, therefore, to favorably shift the therapeutic balance between efficacy and toxicity, with an eye towards quality-of-life outcome metrics. Advancements in material sciences and simultaneous better understanding of molecular pathogenesis and tumor microenvironment have led to the development of novel micrometer-scale DDDs (drug eluting micro-spheres) for locoregional treatment of cancer via transcatheter arterial delivery, under image guidance, as well as nanometer-scale targeted DDSs therapies that work in combination with locally applied energy-deployed drug delivery devices such as radiofrequency $(\mathrm{RF})$, microwave (MW) and high intensity focused ultrasound (HIFU) ablation devices. Some of these technologies, based on a drug plus device plus image guidance, are currently used in the clinic, or being currently evaluated in clinical trials, with varying degrees of integration of the three elements of 1) drug, 2) device, and/or 3) image guidance [4, 7-12].

Nanocarriers have a high surface area to volume ratio, and the surface can be enriched with receptor-targeting moieties or imaging agents for targeted and/ 
or image-guided drug delivery, respectively. In addition, surface modification with polymers providing steric stabilization (e.g. polyethylene glycol (PEG)) can provide an enhanced residence time of these nanocarriers in the bloodstream by opposing uptake by the reticuloendothelial system, which can lead to a higher tumor uptake potentially resulting in increased antitumor activity [13]. For instance, as a single example of receptor-targeting moieties, reports indicate that the use of cyclic Asn-Gly-Arg (cNGR) peptide-targeted nonthermally sensitive liposomes resulted in tumor vasculature destruction, tumor regression, enhanced drug uptake, and improved survival rates in in vivo models of neuroblastoma, lung, and ovarian tumor models [14-16].

Nanocarriers can encapsulate imaging agents in addition to chemotherapeutic drugs [13, 17] enabling monitoring of the whole drug delivery process (pharmacokinetics and tumor accumulation) via noninvasive imaging modalities. This can be used to monitor or optimize delivery, or to refine the treatment algorithm for how to best deliver the combined therapy in an investigational pre-clinical developmental research setting. [18]. Magnetic resonance imaging (MRI) is a non-invasive, non-ionizing, clinical imaging modality used for the visualization of soft tissues including pathological areas such as tumors [19]. MRI contrast agents are used to enhance the visibility of tissue structures and pathologies [20]. Common MRI contrast agents are typically based on gadolinium paramagnetic ions chelated with different chelating agents [20]. Thermosensitive drug-loaded liposomes can co-encapsulate such contrast agents, enabling MRI monitoring of the target accumulation as well as release of the contrast agent from the liposomes, the latter serving in certain cases a surrogate release of drug content, as for example in release triggered by e.g., a localized mild hyperthermia applicator such as RF, MW and HIFU [21, 22]. Thus, spatiotemporal monitoring of the tumor accumulation of drug-loaded nanoparticles can be combined with 
quantification of the extent of cargo release induced by the presence of threshold hyperthermia delivered via MRI-guided applicator devices (e.g., HIFU energy).

DDDs such as drug-eluting beads (microspheres) are delivered via the hepatic artery for the treatment of hepatocellular carcinoma (HCC). This tumor type commonly has enriched arterial supplies via the 'parasitization' of the arterial blood supply during tumorigenesis, when the blood supply ratio from portal vein versus hepatic artery is reversed by the growth of the tumor. The FDAapproved microspheres that are used in TACE are made of anionic constituents (containing $-\mathrm{SO}_{3}^{-}$or $-\mathrm{CO}_{2}{ }^{-}$groups) [23] and are most suitable for carrying cationic anticancer drugs such as doxorubicin [24], topotecan $[25,26]$ and irinotecan [25, 27-39]. In such cases, bead-loading is often facilitated through use of ionic exchange mechanisms. Note that the processes of drug loading and delivery is off label, and not itself FDA-approved, but commonly performed under research settings, preclinically (as mentioned above) or also clinically when 'prescribed' by the research oncologist.

In this thesis work two 'different scale' strategies for the treatment of cancer have been explored:1) NANO-scale: thermosensitive liposomes that target tumor vasculature and imageable thermosensitive liposomes for MR-HIFU therapy (Chapters 2 and 3) and 2) MICRO-scale: imageable microparticles as a drug delivery device (Chapters 4 and 5). Investigation of such strategies has not only therapeutic clinical implications but also can shed light on therapeutic mechanisms and improve our understanding required for the optimization of future drug delivery approaches.

An introduction to existing anticancer treatment strategies is provided in Chapter 1 of this thesis, and our research on image-guided and vascular targeted therapies 
based on nano- and microparticulate approaches is presented in subsequent chapters (Chapters 2-5) followed by concluding remarks (Chapter 6).

Chapter 2 deals with the preparation and characterization of a novel MRI image-able thermosensitive liposome formulation co-loaded with doxorubicin and an MR contrast agent, Gd-HP-DO3A. The developed formulation provided prolonged stability during storage and image-ability during contents release when exposed to mild hyperthermic temperatures. This image-able thermosensitive liposome formulation can be applied in combination with heat sources such as high intensity focused ultrasound (HIFU), radiofrequency (RF), microwave (MW), or laser ablation [40-43] for enhanced drug deposition and monitoring of the fate of delivered drug within the tumor [42-45]. As such, imageable low temperature sensitive liposomes (iLTSLs) presented herein, can find therapeutic utility in systemic tumor-targeted drug delivery possessing the property to report on the specific achieved treatment volume as well as the amount of delivered drug to the tumor.

Monitoring and spatial control of MR-HIFU hyperthermia-induced liposomal content release were demonstrated and moved along the translational pathway utilizing MR-HIFU in tissue-mimicking phantoms followed by in vivo validation. This MR-HIFU guided thermal therapy in combination with iLTSLs may provide correlation of temporal and spatial location of the deployed drug colocalized with signal enhancement on MRI $[46,47]$. These iLTSLs in combination with thermal therapy modalities, including MR-HIFU serve as an instrumental tool towards optimization and understanding of the mechanisms underlying the functioning of a combined drug device therapy and resulting bioeffects. For example, destruction of tumors using this technology may lead to exposure of immune cells to the tumor debris and immune stimulatory substances that are present in the tumor. 
In this example, understanding the mechanisms may lead to the development of new immunotherapeutic treatment strategies.

Chapter 3 reports on the preparation and characterization of novel linear and cyclic NGR peptide motif targeted thermosensitive liposomes (NGR-LTSL). One unique aspect of this work is the development and use of cyclic NGR peptides that do not use a sulfur-sulfur bond for peptide cyclization, as was previously reported [48-54]. Cyclization of peptides with sulfur-sulfur bond is common in peptide chemistry, but the bond is liable for hydrolysis and potentially forms disulfide bridges between adjacent peptides on the liposome surface [55]. These cyclic NGR-targeted LTSLs rapidly ( $>75 \%$ in $<4 \mathrm{sec}$ ) release doxorubicin at $41.3{ }^{\circ} \mathrm{C}$ with minimal drug release at physiological temperature $\left(37^{\circ} \mathrm{C}\right)($ less than $2 \%$ and $5 \%$ over a duration of less than $4 \mathrm{sec}$ and 12 minutes, respectively). As a related follow-up to our work, Kim et al. [56] reported on the formulation of elastin-like polypeptide (ELP)-modified temperature sensitive liposomes (TSL) with the cyclic Arg-Gly-Asp (cRGD) targeting moiety and containing doxorubicin. Results from confocal laser scanning microscopy demonstrate that cRGD-TSL has a 7-fold greater targeting efficiency compared to non-targeted TSL liposomes in a cell line expressing $\mathrm{anb}_{3}$ integrin receptors (endothelial cells). In vivo, approximately 5 times more drug accumulated in the tumor-bearing mice as compared to the non-targeted liposomes. This ELP modified cRGD-TSL-Dox shows slightly lower avidity than we report for cNGR-LTSL-Dox, which have a 10-fold higher avidity for HT-1080 cell line positive for CD13 as compared to ... Similarly, doxorubicin-containing breast cancer-targeted thermosensitive liposomes bearing the HER2-specific affibody have also been formulated and characterized for triggering thermally-induced drug release [57]. An affibody is a small protein molecule engineered to bind to targeted receptor proteins with high affinity in a similar fashion monoclonal antibody does. An in vitro study using these DOX- 
loaded HER2 ${ }^{+}$affisomes (affibody decorated liposomes) demonstrated a 2- to 3-fold higher accumulation of DOX in human breast tumor cells (SK-BR-3) as compared to control nontargeted liposomes [58] confirming the capability of ligand-targeted liposomes to achieve enhanced drug delivery to tumor cells cultured in vitro (vs. nontargeted doxorubicin-containing liposomes).

The in vitro results presented for cNGR-LTSLs herein are encouraging as they demonstrate improved avidity of a cNGR-targeted LTSL for CD13-positive HT1080 cells, as a result of multiple cNGR coupling to the liposome surface. The improved avidity of cNGR-targeted LTSL to vascular receptors achieved by surface attachment of the ligand may translate into increased drug delivery to tumor cells in vivo, as the target vascular receptors are well accessible from the bloodstream. Therefore, further investigation of this approach in a suitable in vivo model is warranted.

Chapters 4 and 5 are concerned with the synthesis of imageable beads (microparticles) for the treatment of hepatocellular carcinoma (HCC) using image-guided transarterial chemoembolization (TACE) with iodine and bismuth as radiopacifiers. Specifically, Chapter $\mathbf{4}$ is focused on the preparation and characterization of iodinated microparticles. These microparticles can load cationic drugs (like, e.g., doxorubicin) via similar mechanisms as reported for drug eluting beads (DEBs) [12, 59], but have the additional advantage of being able to be distinctively visualized using X-ray imaging. In a preclinical study involving hepatic embolization in swine, the visualization of these iodine-containing beads with cone-beam computed tomography (CBCT) was confirmed at an energy dose of $100 \mathrm{kVp}$, a compromise between optimal image-ability and radiation dose [60]. This iodinated microparticle product has achieved US Food and Drug Administration (FDA) approval and has proved its value as a tool to address a 
myriad of pre-clinical and clinical questions. Preliminary clinical results indicate that TACE with iodinated microparticles can be monitored with X-rays and that the bead spatial distribution can be assessed with clinical X-ray imaging techniques; for instance, single shot radiography, fluoroscopy, and/or CBCT. The procedure was very well tolerated in $89 \%$ of the patients $[61,62]$ and appeared useful for targeting tumor tissue at risk of undertreatment $[61,63]$ with minimized off-target embolization [62]. Fusion of imaging and three-dimensional treatment planning tools allowed image-able microsphere mapping and revealing their localization enabling the visualization of tumors at risk for undertreatment which can then be treated in the same or later sessions with catheter- or needlebased local therapy techniques. One limitation, however, is the challenge in distinguishing or differentiating iodinated microparticles from the suspension of iodine-based contrast liquid with clinical CT or CBCT during or post TACE. This can be addressed by waiting till the suspension liquid contrast agent has completely washed out from the vasculature and revisit the post TACE follow up or by developing a method to replace the iodine radiopacifier microparticles with, for example, bismuth radiopacifier microparticles. The latter approach was proposed based on the fact that bismuth and iodine have different k-edge energy absorption, therefore, can be distinguished using clinical dual energy CT. Bismuth is a radiopaque metal with higher k-edge X-ray absorption (90.52 $\mathrm{KeV})$ than iodine $(33.2 \mathrm{KeV})$ [64]. k-edge X-ray absorption is a characteristic energy manifested with a sudden increase in X-ray absorption when the energy of the X-rays is just above the binding energy of the K-shell electrons of the atoms interacting with the incoming photons [65]. Dual energy CT (DECT) is a computed tomography technique, that uses two different energy sources and detectors that are potentially able to distinguish different materials based on differences in k-edge characteristics (Figure 6.1). 


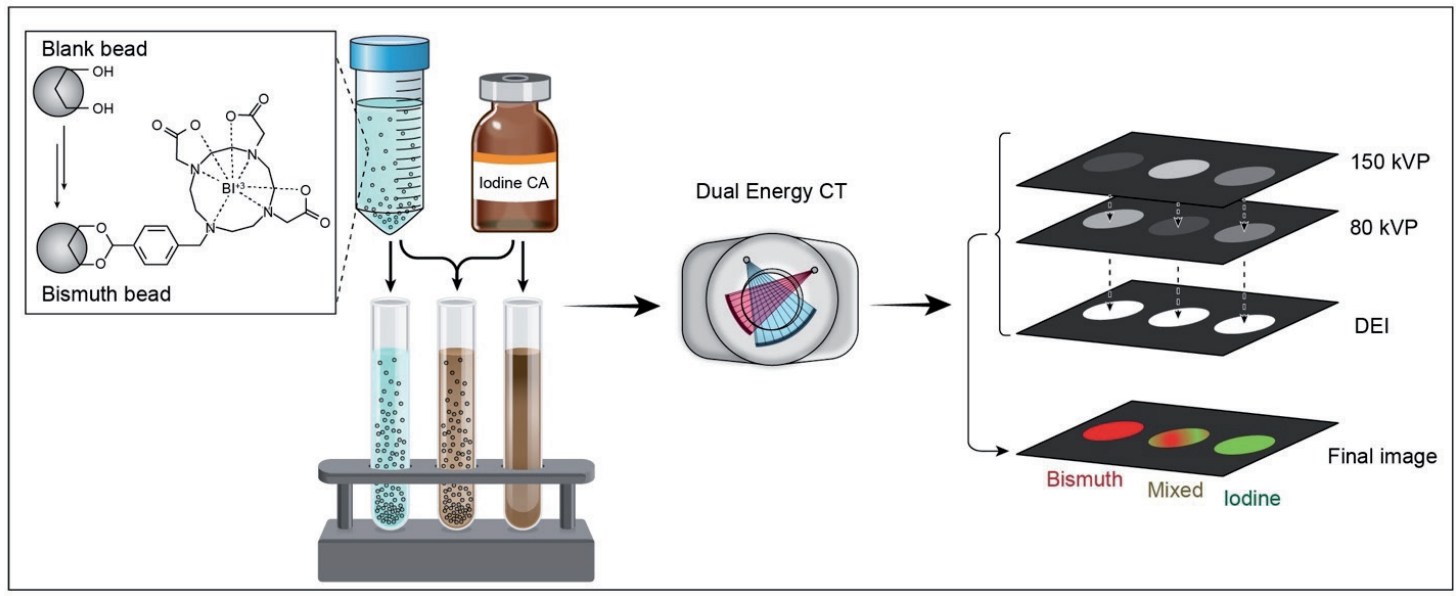

Figure 6.1 Material decomposition using DECT showing separation of bismuth beads from iodine based liquid contrast agent.

Therefore, in Chapter 5, bismuth microparticles were synthesized and characterized for image-ability as well as catheter deliverability and handling. In vitro imageability of bismuth beads was assessed using microCT as well as clinical dual-energy CT (DECT). In DECT, an additional attenuation measurement is obtained with a second X-ray spectrum and detected with either a single detector with different spectral sensitivities or dual detectors, allowing the differentiation of multiple materials based on energy absorption of their K-shell electrons (k-edge energy). Additionally, this also allows quantification of the mass density of two or three materials in a mixture with known elemental composition [65] (Figure 6.1). We observed that our new bismuth-based microparticles are readily distinguishable from the suspending liquid contrast agent (iodine) using DECT when exposed to energy doses of 80 and $150 \mathrm{kVp}$, due to differences in K-edge energy between bismuth and iodine, the enabling feature allowing the follow up during and post TACE of HCC. Based on the chemistry of the bismuth beads, it is found that loading and release profiles expected to be similar with those of its predecessor iodine-based beads. However, further in vivo preclinical studies are required to further explore the radiopacity of these novel beads and assess their embolization, 
handle-ability, compressibility, suspend-ability, as well as drug elution properties in target and non-target tissues.

\section{Future perspectives}

Both bland (non-radiopaque beads) and radiopaque microparticles of specific configurations are generally well able to encapsulate cationic drugs, giving favorable loading properties. However, there are quite some chemotherapeutic drug molecules, useful for the treatment of primary HCC or secondary liver metastases, that are neutral or negatively charged under physiological conditions. Some of these drugs (neutral) often contain diols in their chemical structures, for example capecitabine (Figure 6.2). Therefore, future work should be directed towards functionalizing the microparticles using, for instance, a boronic acid containing moiety to accommodate such drugs (Figure 6.3).

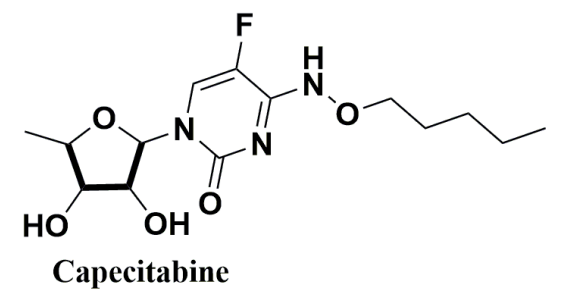

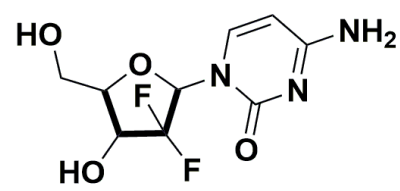

Gemcitabine<smiles>[R4]C(C)[C@@H](O)CC1(S(=O)(=O)C2(CO)CC2)CC1</smiles><smiles>O=C(NC[C@H](O)CO)c1ccncc1Nc1ccc(I)cc1F</smiles>

Pimasertib

Figure 6.2 Examples of anticancer drugs containing diols in their structure 


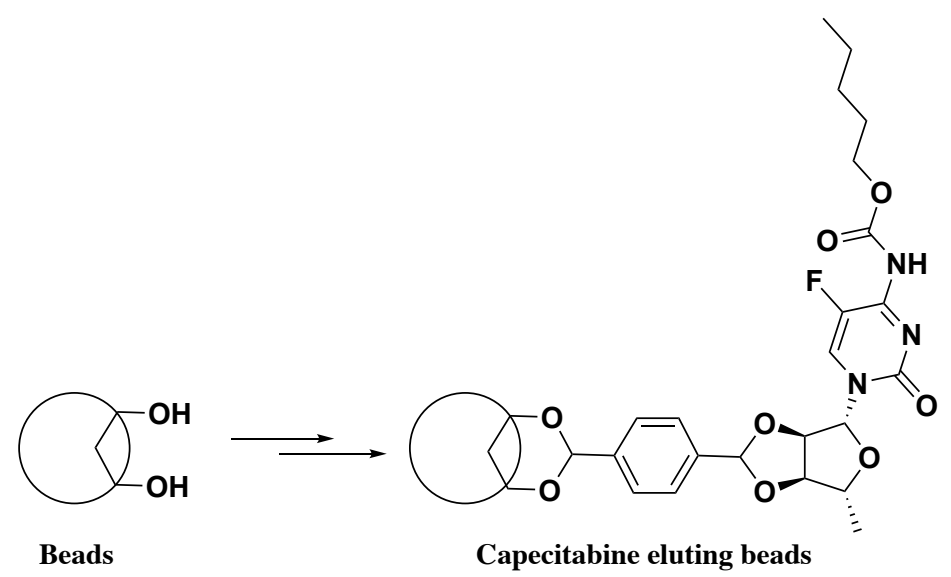

Figure 6.3 A representative model for nonionic drug-eluting beads - capecitabine with its diol group complexed with beads containing a boron moiety to provide capecitabine eluting beads.

Recently, biomedical applications of the use of boronic acid have been proposed in the literature [66-69]. Boronic acid forms cyclic boronated esters in the presence of molecules with 1,2 or 1,3 diols (Figure 6.2). This unique feature of boronic acid can be utilized in bead chemistry in such a way that diol containing anticancer drugs such as capecitabin, gemcitabine, and others. can be conjugated onto the microparticles (Figure 6.3).

Convincing evidence has been obtained showing that boronic acid templates are useful for conjugating drug molecules and that drug release can occur upon changes in the tumor microenvironment $[67,70]$ that induce hydrolysis of the ester bonds. Therefore, beads with boronic acid moieties may become useful to deliver drugs like refametinib, pimasertib and PD-0325901 (MEK inhibitors) $[71,72]$, gossypol [73], and galloflavin (LDH inhibitor) [74], gemcitabine, capecitabine, and other drugs containing diols in their molecular structure (Figure 6.2).

In addition, in case of the use of such neutral drug molecules conjugated into the microparticles, the extent and rate of drug diffusion through tissue might be 
considerably better as compared to the use of positively charged drug molecules as the positively charged drugs will bind electrostatically to negatively charged tissue components. Microparticles adapted to accommodate neutral drugs (but polar due to diols in their structure) by incorporation of a boronic acid moiety could be viewed as a novel drug delivery platform.

\section{Concluding remarks}

The use of targeted nanoparticles and image-able microparticles for delivering anticancer drugs appears attractive for increasing the therapeutic index of anticancer agents following intravenous or locoregional administration. Nanoparticles can be passively targeted and accumulate in tumor tissues through the EPR effect, and/ or actively targeted by surface-exposed targeting ligand binding to receptors over/ selectively expressed on cells and microenvironment components in tumors. On top of these options, as shown in this thesis, drug molecules within thermosensitive nanoparticles can be triggered to be released at the target site with minimally invasive and/or noninvasive image guided thermal therapy devices.

Chemoembolization is often a treatment option for treating HCC when surgery and/or RFA are not suitable, or when conventional anticancer or immunotherapy drugs have failed. Image-guided locoregional therapies have become a clinical standard and are nowadays a vital part of the HCC treatment regimen. These technologies enable estimation of the location and amount of drug delivered to the tumor via drug eluting beads, which is a novel opportunity that potentially can increase the safety of the treatment, and therefore merits further study.

This thesis work represents a combination of material science, chemical synthesis molecular engineering, pharmaceutics and image guided drug delivery. Taken 
together, this thesis addresses the successful formulation of image-guided, targeted and locoregional drug delivery systems based on nanometer- and micrometer-sized particles, respectively.

The nanoparticles are based on thermosensitive liposomes and can be used for: 1) Image-guided tumor delivery of therapeutics in combination with MR-HIFU for monitoring drug contents release. Such temperature-sensitive image-able liposomes can be used for tumor-targeted delivery of therapeutics in combination with MR-HIFU for monitoring of both heating and triggered contents release. This combined use of intravenously administered temperaturesensitive liposomes with MR-HIFU has shown to result in increased intra-tumoral drug concentrations when compared to treatment with either free drug or drugcontaining liposomes which are not temperature sensitive. This technology has great potential for the treatment of patients with solid tumors accessible for MR-HIFU exposure. 2) Actively targeted drug delivery to endothelial cells in the tumor vasculature, as exemplified in this thesis with the developed cNGRLTSLs containing doxorubicin. This thermosensitive, active targeting system can be applied for targeting drugs to the tumor vasculature of solid tumors and their metastases. The vasculature targeting feature is hoped to enable the retention of these liposomes at the tumor vasculature long enough to optimally utilize hyperthermia treatment.

The micro-sized particles in this thesis are radiopaque drug-eluting microparticles containing iodine or bismuth radiopacifiers which were successfully synthesized by direct chemical conjugation of a radio-dense triiodo phenyl species or bismuth chelated macrocycle to commercially available microparticles and which are also able to carry cationic drugs like doxorubicin. These image-able embolic microparticles are visible under fluoroscopy and CBCT which enables real- 
time feedback of bead location (and thus localization of drug and therapeutic ischemic effect) during and after DEB-TACE. This property makes the beads useful in providing physicians with feedback on TACE and enables identification of undertreated tissue. Iodinated microparticles report on the amount of delivered drug and drug distribution in the liver post TACE, as bead (drug source) location is directly correlated with the CT image intensity. As opposed to the iodinated beads, the bismuth beads are differentiable from the medium containing iodine clinical contrast agent used to suspend the beads during catheterization using DECT. Drug containing bismuth beads can also be delivered in combination with drug containing iodine beads (dual bead-dual drug approach) in the aim to deliver drug combinations to bring therapeutic synergy. The individual beads and drug distribution can be assessed using DCET as DECT provides material decomposition ability. The novel findings in this thesis are the preparation of the two microparticle systems, the catheter deliverability, the beads image-ability with microCT, CT and differentiability among different radiopaque materials using DECT. Therefore, the initial essential steps have been realized and pave the way for successful application of this paradigm.

The ability to image the drug-eluting beads during or post TACE is important as it provides physicians with: 1) real-time information regarding the spatial distribution of the beads in order to avoid off-target embolization and to inform on on-target beads. Off-target embolization has a risk of embolizing vessels feeding the normal liver which could be minimized with this technology while information on on-target embolization helps to optimize the quality of the treatment which may lead to better efficacy. 2) definition of standardized and reproducible endpoints for the chemoembolization procedure, 3) estimation, from the beads localization pattern, of the spatial drug localization and drug amount delivered at the end of the procedure and 4) real-time feedback for additional procedural intervention, if 
necessary, based on the beads localization pattern, and 5) quantifiable endpoints for reproducible technique normalization, such that the TACE procedure could become more standardized and less subjectively operator dependent. 


\section{References}

1. Aftab, S., et al., Nanomedicine: an effective tool in cancer therapy. I. J. Pharm., 2018. 540(12): p. 132-149.

2. Jabir, N.R., et al., An overview on the current status of cancer nanomedicines. Curr. Med. Res. Opin., 2018. 34(5): p. 911-921.

3. Jain, V., S. Jain, and S.C. Mahajan, Nanomedicines based drug delivery systems for anti-cancer targeting and treatment. Curr. Drug Deliv., 2015. 12(2): p. 177-191.

4. Tong, R. and J.J. Cheng, Anticancer polymeric nanomedicines. Polym. Rev., 2007. 47(3): p. 345-381.

5. Svenson, S., What nanomedicine in the clinic right now really forms nanoparticles? Wiley Interdiscip. Rev. Nanomed. Nanobiotechnol., 2014. 6(2): p. 125-135.

6. Moles, E. and M. Kavallaris, A potent targeted cancer nanotherapeutic. Nat. Biomed. Eng., 2019. 3(4): p. 248-250.

7. Ferrari, M., Beyond drug delivery. Nat. Nanotechnol., 2008. 3(3): p. 131-132.

8. Duncan, R., Polymer conjugates as anticancer nanomedicines. Nat. Rev. Cancer, 2006. 6(9): p. 688-701.

9. Venditto, V.J. and F.C. Szoka, Cancer nanomedicines: so many papers and so few drugs! Adv. Drug Deliv. Rev., 2013. 65(1): p. 80-88.

10. Dawidczyk, C.M., et al., State-of-the-art in design rules for drug delivery platforms: lessons learned from FDA-approved nanomedicines. J. Control. Release, 2014. 187: p. 133-144.

11. Farokhzad, O.C. and R. Langer, Impact of nanotechnology on drug delivery. ACS. Nano, 2009. 3(1): p. 16-20.

12. Lewis, A.L., et al., Bench-to-clinic development of imageable drug-eluting embolization beads: finding the balance. Future Oncol., 2018. 14(26): p. 2741-2760.

13. Hong, S., et al., The binding avidity of a nanoparticle-based multivalent targeted drug delivery platform. Chem. Bio., 2007. 14(1): p. 107-115.

14. Pastorino, F., et al., Enhanced antitumor efficacy of clinical-grade vasculature-targeted liposomal doxorubicin. Clin. Cancer Res., 2008. 14(22): p. 7320-7329.

15. Pastorino, F., et al., Targeting liposomal chemotherapy via both tumor cell-specific and tumor vasculature-specific ligands potentiates therapeutic efficacy. Cancer Res., 2006. 66(20): p. 10073-10082.

16. Pastorino, F., et al., Vascular damage and anti-angiogenic effects of tumor vessel-targeted liposomal chemotherapy. Cancer Res., 2003. 63(21): p. 7400-7409.

17. Danhier, F., O. Feron, and V. Préat,. To exploit the tumor microenvironment: passive and active tumor targeting of nanocarriers for anti-cancer drug delivery. J. Control. Release, 2010. 148(2): p. 135-146.

18. Lamichhane, N., et al., Liposomes: clinical applications and potential for image-guided drug delivery. Molecules, 2018. 23(2): p. 288/1-288/17.

19. Barisano, G., et al., Clinical 7 T MRI: are we there yet? a review about magnetic resonance imaging at ultra-high field. Brit. J. Radiol., 2019. 92(1094).

20. Nitz, W.R., Magnetic Resonance Imaging. Springer Handbook of Medical Technology, 2011: p. 439-460.

21. Ta, T. and T.M. Porter, Thermosensitive liposomes for localized delivery and triggered release of chemotherapy. J. Control. Release, 2013. 169(1-2): p. 112-125.

22. Man, F., et al., Imaging nanomedicine-based drug delivery: a review of clinical studies. Mol Imaging Biol, 2018. 20(5): p. 683-695. 
23. de Baere, T., et al., An in vitro evaluation of four types of drug-eluting microspheres loaded with doxorubicin. J. Vasc. Interv. Radiol., 2016. 27(9): p. 1425-1431.

24. Doucet, J., et al., Advances in degradable embolic microspheres: a state of the art review. J. Funct. Biomater., 2018. 9(1): p. 14/1-14/24.

25. Forster, R.E., et al., Comparison of DC bead-irinotecan and DC bead-topotecan drug eluting beads for use in locoregional drug delivery to treat pancreatic cancer. J. Mater. Sci. Mater. Med., 2010. 21(9): p. 2683-90.

26. Marr, B.P., et al., Three-drug intra-arterial chemotherapy using simultaneous carboplatin, topotecan and melphalan for intraocular retinoblastoma: preliminary results. Brit. J. Ophthalmol., 2012. 96(10): p. 1300-3.

27. Akinwande, O., C. Scoggins, and R.C.G. Martin, Early experience with 70-150 micrometer (M1) irinotecan drug-eluting beads (M1-DEBIRI) for the treatment of unresectable hepatic colorectal metastases. Anticancer Res., 2016. 36(7): p. 3413-3418.

28. Akinwande, O.K., et al., Small versus large-sized drug-eluting beads (DEBIRI) for the treatment of hepatic colorectal metastases: a propensity score matching analysis. Cardiovas. Interv. Radiol., 2015. 38(2): p. 361-371.

29. Aliberti, C., et al., Trans-arterial chemoembolization of metastatic colorectal carcinoma to the liver adopting DC bead, drug-eluting bead loaded with irinotecan: results of a phase II clinical study. Anticancer Res., 2011. 31(12): p. 4581-4587.

30. Ashrafi, K., et al., Characterization of a novel intrinsically radiopaque drug-eluting bead for image-guided therapy: DC bead LUMI ${ }^{\mathrm{TM}}$. J. Control. Release, 2017. 250: p. 36-47.

31. Baltes, S., et al., Doxorubicin and irinotecan drug-eluting beads for treatment of glioma: a pilot study in a rat model. J. Mater. Sci. Mater. Med., 2010. 21 (4): p. 1393-402.

32. Bhutiani, N., O. Akinwande, and R.C.G. Martin, Efficacy and toxicity of hepatic intra-arterial drug-eluting (Irinotecan) bead (DEBIRI) therapy in irinotecan-refractory unresectable colorectal liver metastases. World J. Surg., 2016. 40(5): p. 1178-1190.

33. Fiorentini, G., et al., Trans-arterial chemoembolization of metastatic colorectal carcinoma $(M C R C)$ to the liver adopting polyvinyl alcohol microspheres (PAM) loaded with irinotecan compared with FOLFIRI (CT): evaluation at two years of a phase III clinical trial. Ann. Oncol., 2010. 21: p. 191-191.

34. Swaine, T., et al., Evaluation of ion exchange processes in drug-eluting embolization beads by use of an improved flow-through elution method. Eur. J. Pharm. Sci., 2016. 93: p. 351-359.

35. Tang, Y., et al., Evaluation of irinotecan drug-eluting beads: a new drug-device combination product for the chemoembolization of hepatic metastases. J. Control. Release, 2006. 116(2): p. E55-E56.

36. Taylor, R.R., et al., Irinotecan drug eluting beads for use in chemoembolization: in vitro and in vivo evaluation of drug release properties. Eur. J. Pharm. Sci., 2007. 30(1): p. 7-14.

37. Venturini, M., et al., Chemoembolization with drug eluting beads preloaded with irinotecan (DEBIRI) vs doxorubicin (DEBDOX) as a second line treatment for liver metastases from cholangiocarcinoma: a preliminary study. Brit.J. Radiol., 2016. 89(1067).

38. Yan, J., et al., Preparation and characterization of irinotecan loaded cross-linked bovine serum albumin beads for liver cancer chemoembolization therapy. Int. J. Polym. Sci., 2016.

39. Young, S., et al., Review of the clinical evidence for the use of DEBIRI in the treatment of colorectal metastatic disease. Cardiovasc. Interv. Radiol., 2017. 40(4): p. 496-501.

40. Dromi, S., et al., Pulsed-high intensity focused ultrasound and low temperature sensitive liposomes for enhanced targeted drug delivery and antitumor effect. Clin. Cancer Res., 2007. 13(9): p. 2722-2727. 
41. Swenson, C.E., et al., Increased duration of heating boosts local drug deposition during radiofrequency ablation in combination with thermally sensitive liposomes (ThermoDox) in a porcine model. PLoS One, 2015. 10(10): p. e0139752/1-e0139752/15.

42. Dou, Y.N., et al., Custom-designed laser-based heating apparatus for triggered release of cisplatin from thermosensitive liposomes with magnetic resonance image guidance. J. Visualized Exp., 2015(106): p. e53055/1-e53055/11.

43. Hijnen, N., et al., Thermal combination therapies for local drug delivery by magnetic resonanceguided high-intensity focused ultrasound. Proc. Natl. Acad. Sci. U. S. A., 2017. 114(24): p. E4802-E4811.

44. Ponce, A., et al., Magnetic resonance imaging of temperature-sensitive liposome release: Drug dose painting and antitumor effects. J. Nat. Cancer Inst., 2007. 99(1): p. 53-63.

45. Ponce, A.M., et al., Targeted bioavailability of drugs by triggered release from liposomes. Future Lipidol., 2006. 1(1): p. 25-34.

46. Hijnen, N., S. Langereis, and H. Gruell, Magnetic resonance guided high-intensity focused ultrasound for image-guided temperature-induced drug delivery. Adv. Drug Deliv. Rev., 2014. 72: p. 65-81.

47. Fernando, R., et al., MRI-guided monitoring of thermal dose and targeted drug delivery for cancer therapy. Pharm. Res., 2013. 30(11): p. 2709-2717.

48. Pasqualini, R., et al., Aminopeptidase $N$ is a receptor for tumor-homing peptides and a target for inhibiting angiogenesis. Cancer Res., 2000. 60(3): p. 722-727.

49. Garde, S.V., et al., Binding and internalization of NGR-peptide-targeted liposomal doxorubicin (TVT-DOX) in CD13-expressing cells and its antitumor effects. Anti-Cancer Drugs, 2007. 18(10): p. 1189-1200.

50. Buehler, A., et al., cNGR: A novel homing sequence for CD13/APN targeted molecular imaging of murine cardiac angiogenesis in vivo. Arterioscler Thromb. Vasc. Bio., 2006. 26(12): p. 2681-2687.

51. Curnis, F., et al., Differential binding of drugs containing the NGR motif to CD13 isoforms in tumor vessels, epithelia, and myeloid cells. Cancer Res., 2002. 62(3): p. 867-874.

52. Majhen, D., et al., Disulfide bond formation in NGR fiber-modified adenovirus is essential for retargeting to aminopeptidase $N$. Biochemical and Biophysical Research Communications, 2006. 348(1): p. 278-287.

53. van Hensbergen, Y., et al., A doxorubicin-CNGRC-peptide conjugate with prodrug properties. Biochem. Pharmacol., 2002. 63(5): p. 897-908.

54. von Wallbrunn, A., et al., In vivo optical imaging of CD13/APN-expression in tumor xenografts. J. Biomed. Optics, 2008. 13(1): p. 9.

55. Corti, A. and M. Ponzoni, Tumor vascular targeting with tumor necrosis factor alpha and chemotherapeutic drugs. Ann. N Y., acad. Sci., 2004. 1028: p. 104-112.

56. Kim, M.S., et al., Temperature-triggered tumor-specific delivery of anticancer agents by cRGDconjugated thermosensitive liposomes. Colloids Surf., B, 2014. 116: p. 17-25.

57. Puri, A., et al., HER2-Specific Affibody-Conjugated Thermosensitive Liposomes (Affisomes) for Improved Delivery of Anticancer Agents. J. Liposome Res., 2008. 18(4): p. 293-307.

58. Smith, B., et al., Hyperthermia-triggered intracellular delivery of anticancer agent to HER2+ cells by HER2-specific affibody (ZHER2-GS-Cys)-conjugated thermosensitive liposomes (HER2+ affisomes). J. Control. Release, 2011. 153(2): p. 187-194.

59. Hagan, A., et al., Preparation and characterization of vandetanib-eluting radiopaque beads for locoregional treatment of hepatic malignancies. Eur. J. Pharm. Sci., 2017. 101: p. 22-30. 
60. Pritchard, W.F., et al. Hepatic arterial embolization with radiopaque microbeads containing iodine or bismuth in swine: high resolution imaging and material decomposition with photoncounting CT. in Workshop on Medical Applications of Spectroscopic X-ray Detectors. 2019. Switzerland: CERN.

61. Aliberti, C., et al., Hepatic Arterial Infusion of Polyethylene Glycol Drug-eluting Beads for Primary and Metastatic Liver Cancer Therapy. Anticancer Research, 2016. 36(7): p. 35153521.

62. Lencioni, R., et al., Transarterial chemoembolization (TACE) of hepatocellular carcinoma (HCC) with a novel radiopaque (RO) drug eluting bead (DEB). J. Clin. Oncol., 2018. 36(4).

63. Levy, E.B., et al., First Human Experience with Directly Image-able Iodinated Embolization Microbeads. Cardiovasc. Interv. Radiol., 2016. 39(8): p. 1177-1186.

64. Alegethami, M.B., Anton; Feltis, Bryce; Geso, Moshi, Bismuth Sulfide Nanoparticles as a Complement to Traditional Iodinated Contrast Agents at Various X-Ray Computed Tomography Tube Potentials. J. Nanomater. Mole. Nanotechnol., 2017. 6(6): p. 9.

65. He, P., et al., Material discrimination based on k-edge characteristics. Comput Math. Methods Med., 2013. 2013: p. 308520.

66. Ban, H.S. and H. Nakamura, Boron-Based Drug Design. Chem. Rec., 2015. 15(3): p. 616635.

67. Chen, J.X., et al., One-pot construction of boronate ester based pH-responsive micelle for combined cancer therapy. Colloid. Surface. B., 2016. 143: p. 285-292.

68. Vancoillie, G. and R. Hoogenboom, Responsive Boronic Acid-Decorated (Co)polymers: From Glucose Sensors to Autonomous Drug Delivery. Sensors, 2016. 16(10).

69. Yang, W.Q., X.M. Gao, and B.H. Wang, Boronic acid compounds as potential pharmaceutical agents. Med. Res. Rev., 2003. 23(3): p. 346-368.

70. Wang, Y.X., et al., pH-and glucose-sensitive glycopolymer nanoparticles based on phenylboronic acid for triggered release of insulin. Carbohydr. Polym., 2012. 89(1): p. 124-131.

71. Cheng, Y. and H.Q. Tian, Current Development Status of MEK Inhibitors. Molecules, 2017. 22(10).

72. Adjei, A.A., The role of mitogen-activated ERK-kinase inhibitors in lung cancer therapy. Clin. Lung Cancer, 2005. 7(3): p. 221-223.

73. Keshmiri-Neghab, H. and B. Goliaei, Therapeutic potential of gossypol: An overview. Pharm. Biol. (London, U. K.), 2014. 52(1): p. 124-128.

74. Manerba, M., et al., Galloflavin (CAS 568-80-9): A Novel Inhibitor of Lactate Dehydrogenase. Chem. Med. Chem., 2012. 7(2): p. 311-317. 



\section{Samenvatting}

De drie belangrijkste behandelingsmethoden voor kanker zijn chirurgie, radiotherapie en chemotherapie [1-6]. Helaas gaan deze methoden maar al te vaak gepaard met een hoog risico op letsel of toxiciteit voor gezonde weefsels. Daarom worden meer selectieve behandelingsparadigma's en adjuvante combinatiemethoden onderzocht, die tumorcellen kunnen vernietigen met minder of geen neveneffecten, waaronder immunotherapie, drug delivery systems (DDS), en drug delivery devices (DDD). Idealiter zal een combinatie van twee of meer van deze strategieën nog selectievere behandelingen met een betere veiligheid en werkzaamheid van de therapie mogelijk maken. Eén van deze nieuwe benaderingen omvat de combinatie van DDS met minimaal of niet-invasieve hulpmiddelen voor warmteontwikkeling, die de vrijgave van het geneesmiddel uit de DDS bevorderen of de lokalisatie van DDS in kwaadaardig weefsel verhogen, om de therapeutische index van het geneesmiddel te maximaliseren. Deze combinatie is gericht op een gunstige verschuiving van het therapeutisch evenwicht tussen werkzaamheid en toxiciteit, met het oog op (behoud van) kwaliteit van het leven. Vooruitgang in material science en een beter inzicht in de moleculaire pathogenese en de tumor micro-omgeving, hebben geleid tot de ontwikkeling van nieuwe drug eluting microspheres voor de locoregionale behandeling van kanker door middel van arteriële toediening via een katheter onder beeldgeleiding, alsook gerichte DDS-therapieën op nanometerschaal. Deze werken in combinatie met lokaal toegepaste, van energie voorziene toedieningsapparaten voor geneesmiddelen, zoals radiofrequentie- (RF), microgolf- (MW) en ultrasone systemen met hoge intensiteit (HIFU, high intensity focused ultrasound) voor thermische ablatie. Imageguided drug delivery wordt momenteel gebruikt in de kliniek, of wordt geëvalueerd 
in klinische studies, met verschillende graden van integratie van de drie elementen van 1) geneesmiddel, 2) apparaat, en/of 3) beeldgeleiding [4, 7-12].

Nanodragers hebben een hoog oppervlakte-volumeverhouding; het oppervlak kan verrijkt worden met receptor-targeting deeltjes of beeldvormende middelen voor respectievelijk gerichte en/of beeldgestuurde medicijnafgifte. Bovendien kan oppervlaktemodificatie met polymeren, die zorgen voor sterische stabilisatie (zoals polyethyleenglycol (PEG)), zorgen voor een langere retentietijd van deze nanodragers in de bloedbaan, door opname door het reticulo-endotheliale systeem (RES) tegen te gaan. Dit kan leiden tot een hogere tumor opname, die mogelijk resulteert in een verhoogde anti-tumoractiviteit [13]. Als voorbeeld van receptor-targeting deeltjes: uit studies blijkt dat het gebruik van cyclische Asn-GlyArg (cNGR) peptide-gerichte niet-thermisch gevoelige liposomen resulteert in vernietiging van de tumor vasculatuur, tumorregressie, verhoogde geneesmiddel opname, en verbeterde overlevingskansen in in vivo modellen van neuroblastoom, long, en ovariumtumor [14-16].

Nanodragers kunnen naast cytostatica ook beeldvormende middelen inkapselen $[13,17]$, waardoor het hele toedieningsproces van het geneesmiddel (farmacokinetiek en tumoraccumulatie) gevolgd kan worden via niet-invasieve beeldvormingsmodaliteiten. Dit kan worden gebruikt om de toediening te controleren of te optimaliseren, of om het behandelingsalgoritme te verfijnen en overbeteren in een setting voor preklinisch ontwikkelingsonderzoek. [18]. Magnetic resonance imaging (MRI) is een niet-invasieve, niet-ioniserende, klinische beeldvormingsmodaliteit, die wordt gebruikt voor de visualisatie van zachte weefsels, met inbegrip van pathologische gebieden zoals tumoren [19]. MRIcontrastmiddelen worden gebruikt om de zichtbaarheid van weefselstructuren en pathologieën te verbeteren [20]. MRI-contrastmiddelen zijn meestal gebaseerd 
op gadolinium complexen [20]. Thermo-sensitieve liposomen kunnen dergelijke contrastmiddelen inkapselen, waardoor MRI-monitoring van de accumulatie mogelijk wordt, evenals afgifte van het contrastmiddel uit de liposomen; dit laatste dient in bepaalde gevallen als surrogaatafgifte van de geneesmiddelinhoud, zoals bijvoorbeeld bij afgifte door een gelokaliseerde milde hyperthermieapplicator zoals RF, MW en HIFU [21,22]. Derhalve kan spatio-temporele monitoring van de tumoraccumulatie van DDS worden gecombineerd met kwantificering van de mate van vrijkomen van lading geïnduceerd door de aanwezigheid van hyperthermie toegediend via MRI-geleide applicatorapparaten (bijvoorbeeld HIFU).

DDDs zoals drug-eluting beads (microsferen) worden via de leverslagader toegediend voor de behandeling van hepatocellulair carcinoom (HCC). Dit tumortype heeft gewoonlijk een verrijkte arteriële toevoer via de "parasitering" van de arteriële bloedtoevoer tijdens de tumorigenese, wanneer de verhouding bloedtoevoer vanuit de poortader versus de leverslagader wordt omgekeerd door de groei van de tumor. De door de US Food and Drug Administration (FDA) goedgekeurde microsferen die bij TACE (Transarteriële chemoembolizatie) worden gebruikt zijn gemaakt van anionische bestanddelen [23] en zijn het meest geschikt voor het dragen van kationische anti-kankermedicijnen zoals doxorubicine [24], topotecan [25, 26] en irinotecan [25, 27-39]. In dergelijke gevallen wordt het laden vaak vergemakkelijkt door gebruik te maken van ionische uitwisselingsmechanismen. De processen van het laden en afleveren van geneesmiddelen zijn off-label, en niet door de FDA goedgekeurd, en worden doorgaans uitgevoerd in een preklinische setting.

In dit proefschrift zijn twee strategieën voor de behandeling van kanker onderzocht: i) NANO-schaal: thermo-sensitieve liposomen die zich richten op de tumor vasculatuur en imageable thermo-sensitieve liposomen voor MR-HIFU 
therapie (Hoofdstukken 2 en 3) en ii) MICRO-schaal: imageable microdeeltjes als drug delivery device (Hoofdstukken 4 en 5). Onderzoek naar dergelijke strategieën heeft niet alleen therapeutische klinische implicaties, maar kan ook licht werpen op therapeutische mechanismen en ons begrip verbeteren dat nodig is voor de optimalisatie van toekomstige geneesmiddelentoedieningsbenaderingen.

Een inleiding tot bestaande strategieën voor de behandeling van kanker wordt gegeven in hoofdstuk 1 van dit proefschrift. Ons onderzoek naar beeldgeleide en vasculair gerichte therapieën gebaseerd op nano- en microdeetjes benaderingen wordt gepresenteerd in de daaropvolgende hoofdstukken (hoofdstukken 2-5) gevolgd door concluderende opmerkingen (hoofdstuk 6).

Hoofdstuk 2 behandelt de bereiding en karakterisering van een nieuwe thermo-sensitieve liposoom formulering, geladen met doxorubicine en een MR contrastmiddel (Gd-HP-DO3A). De ontwikkelde liposomale formulering bood langdurige stabiliteit tijdens opslag en beeldvorming tijdens afgifte van de inhoud bij blootstelling aan milde hyperthermische condities. Deze beeldgevoelige thermo-sensitieve liposoomformulering kan worden toegepast in combinatie met warmtebronnen zoals hoge intensiteit gefocusseerd ultrageluid (HIFU), radiofrequentie $(\mathrm{RF})$, microgolf $(\mathrm{MW})$, of laserablatie [40-43] voor verbeterde geneesmiddelafgifte en monitoring van het toegediende geneesmiddel in de tumor [42-45]. Imageable lage temperatuur-gevoelige liposomen (iLTSLs) kunnen therapeutisch toepassing vinden in systemische toediening van tumor gerichte geneesmiddelen met informatie over het specifieke bereikte behandelingsvolume en de hoeveelheid toegediende geneesmiddel aan de tumor.

Monitoring en ruimtelijke controle van MR-HIFU hyperthermie-geïnduceerde afgifte van liposomale inhoud werden gedemonstreerd en verder ontwikkeld op het 
translationele pad met behulp van in vitro MR-HIFU experimenten gevolgd door in vivo validatie. Deze door MR-HIFU geleide thermische therapie in combinatie met iLTSLs kan een correlatie opleveren van de temporele en ruimtelijke locatie van het toegediende geneesmiddel, co-lokalisatie met signaalversterking op MRI $[46,47]$. Deze iLTSLs in combinatie met thermische therapie modaliteiten, zoals MR-HIFU, dienen als een instrument voor de optimalisatie en het begrip van de mechanismen die ten grondslag liggen aan de werking van een gecombineerde drug device therapie en de daaruit voortvloeiende biologische effecten. De vernietiging van tumoren met behulp van deze technologie zou kunnen leiden tot blootstelling van immuuncellen aan de tumorresten en immuunstimulerende stoffen die in de tumor aanwezig zijn. Het begrijpen van deze mechanismen kan leiden tot de ontwikkeling van nieuwe immunotherapeutische behandelingsstrategieën.

In hoofdstuk 3 wordt onderzoek gedaan naar de bereiding en karakterisering van nieuwe lineaire en cyclische NGR peptide sequenties gerichte thermosensitieve liposomen (NGR-LTSL). Een uniek aspect van dit werk is de ontwikkeling en het gebruik van cyclische NGR peptiden, die geen gebruik maken van een zwavelzwavel binding voor peptide cyclisatie, zoals eerder werd gerapporteerd [48-54]. Deze zwavel-zwavel binding is gevoelig voor hydrolyse en mogelijk gevoelig voor de vorming van disulfide bruggen tussen aangrenzende peptiden op het liposoom oppervlak [55].

Deze cyclische NGR-gerichte LTSLs geven snel doxorubicine af bij $41,3^{\circ} \mathrm{C}(>75 \%$ in $<4 \mathrm{sec})$ met minimale geneesmiddelafgifte bij fysiologische temperatuur $\left(37^{\circ} \mathrm{C}\right)$ (minder dan 2\% en 5\% over een duur 4 sec en 12 minuten, respectievelijk). Als een vervolg op ons werk rapporteerden Kim et al. [56] over de formulering van elastine-achtige polypeptide (ELP)-gemodificeerde temperatuurgevoelige liposomen (TSL) met het cyclische Arg-Gly-Asp (cRGD) targeting gedeelte, die 
doxorubicine bevatten. Resultaten van confocale laserscanmicroscopie tonen aan dat cRGD-TSL een 7-maal hogere targeting-efficiëntie heeft dan non-targeted TSL-liposomen in een cellijn die drie integrine receptoren tot expressie brengt (endotheelcellen). In vivo accumuleerde ongeveer vijfmaal meer geneesmiddel in de tumordragende muizen in vergelijking met de non-targeted liposomen. Deze ELP gemodificeerde cRGD-TSL-Dox vertoont een iets lagere aviditeit dan cNGR-LTSL-Dox, die een 10-voudige hogere aviditeit hebben voor HT-1080 cellijn positief voor CD13. Op dezelfde manier werden doxorubicine-bevattende borstkanker gerichte thermosensitieve liposomen met HER2-specifieke affibody geformuleerd en gekarakteriseerd voor thermisch geïnduceerde afgifte van geneesmiddelen [57]. Een affibody is een klein eiwitmolecuul dat ontworpen is om zich met hoge affiniteit te binden aan de receptoreiwitten van het doelwit, op een vergelijkbare manier als monoklonale antilichamen dat doen. Een in vitro studie met deze met DOX beladen HER ${ }^{2+}$ affisomen (affibody-gedecoreerde liposomen) toonde een 2 tot 3 maal hogere accumulatie van DOX aan in menselijke borsttumorcellen (SK-BR-3) in vergelijking met niet-gerichte controleliposomen [58]. Dit bevestigt dat ligand-gerichte liposomen in staat zijn om een verbeterde geneesmiddelafgifte aan in vitro gekweekte tumorcellen te bereiken (in vergelijking met niet-gerichte doxorubicine-bevattende liposomen).

De in vitro resultaten voor cNGR-LTSLs zijn bemoedigend, omdat ze een verbeterde aviditeit van een cNGR-LTSL voor CD13 ${ }^{+}$HT-1080 cellen aantonen, als gevolg van meervoudige koppeling van cNGR's aan het liposoomoppervlak. De verbeterde aviditeit van cNGR-gerichte LTSL voor vasculaire receptoren, bereikt door koppeling van het ligand aan het oppervlak, kan zich vertalen in een verhoogde medicijnafgifte aan tumorcellen in vivo, aangezien de vasculaire doelreceptoren goed toegankelijk zijn vanuit de bloedbaan. Daarom is verder onderzoek van deze benadering in een geschikt in vivo model gerechtvaardigd. 
De hoofdstukken 4 en $\mathbf{5}$ hebben betrekking op de synthese van imageable microdeeltjes voor de behandeling van hepatocellulair carcinoom (HCC) met behulp van beeldgeleide TACE met jodium en bismut als radiopacifiers. Hoofdstuk 4 zicht op de bereiding en karakterisering van gejodeerde microdeeltjes. Deze microdeetjes kunnen kationische geneesmiddelen laden (zoals bijvoorbeeld doxorubicine) via vergelijkbare mechanismen als gerapporteerd voor drug eluting beads (DEBs) [12, 59], maar hebben als bijkomend voordeel dat ze duidelijk gevisualiseerd kunnen worden met behulp van röntgenbeeldvorming. In een preklinisch onderzoek met leverembolisatie bij varkens werd de visualisatie van deze jodiumhoudende deeltjes met cone-beam computertomografie (CBCT) bevestigd bij een energiedosis van $100 \mathrm{kV}$, een compromis tussen optimale beeldvorming en stralingsdosis [60]. Dit product met gejodeerde microdeeltjes heeft de goedkeuring gekregen van de FDA en heeft zijn waarde bewezen, doordat het hielp een groot aantal (pre)klinische vragen te beantwoorden. Voorlopige klinische resultaten wijzen erop dat TACE met gejodeerde microdeeltjes kan worden gevolgd met röntgenstralen en dat de ruimtelijke verdeling van de microdeeltjes kan worden beoordeeld met klinische röntgenbeeldvormingstechnieken; bijvoorbeeld single shot radiografie, fluoroscopie, en/of CBCT. De procedure werd zeer goed verdragen bij $89 \%$ van de patiënten $[61,62]$ en bleek nuttig voor het richten van tumorweefsel met risico op onderbehandeling [61, 63] met minimale off-target embolisatie [62]. Fusie van beeldvorming en drie-dimensionale behandeling planning tools om imageable microdeeltjes in kaart brengen en het onthullen van hun lokalisatie waardoor de visualisatie van tumoren met een risico voor onderbehandeling die vervolgens kunnen worden behandeld in dezelfde of latere sessies met katheter of naald-gebaseerde lokale therapie technieken.

Het blijft echter een uitdaging om jodiumhoudende microdeeltjes te onderscheiden of te onderscheiden van de suspensie van jodiumhoudende 
contrastvloeistof met klinische CT of CBCT tijdens of na TACE. Dit kan worden aangepakt door te wachten tot de suspensie contrastvloeistof volledig uit de vasculatuur is weggespoeld en de follow-up na TACE opnieuw te verrichten, of door een methode te ontwikkelen om de jodiumhoudende microdeeltjes te vervangen door bijvoorbeeld bismuthoudende microdeeltjes. Deze laatste aanpak werd voorgesteld op basis van het feit dat bismut en jodium een verschillende $k$-edge energieabsorptie hebben en daarom met klinische dubbel-energetische CT kunnen worden onderscheiden. Bismut is een radiopaak metaal met een hogere $k$-edge röntgenabsorptie $(90,52 \mathrm{KeV})$ dan jodium $(33,2 \mathrm{KeV})$ [64]. $K$-edge röntgenabsorptie is een kenmerkende energie die zich manifesteert met een plotselinge toename van röntgenabsorptie wanneer de energie van de röntgenstraling net boven de bindingsenergie ligt van de K-shell elektronen van de atomen die interageren met de inkomende fotonen [65]. Dual energy CT (DECT) is een computertomografietechniek, waarbij gebruik wordt gemaakt van twee verschillende energiebronnen en detectoren, die potentieel in staat zijn verschillende materialen te onderscheiden op basis van verschillen in $k$-edge kenmerken (figuur 6.1).

In hoofdstuk 5 werden bismut microdeeltjes gesynthetiseerd en gekarakteriseerd voor zowel de beeldvorming als de leverbaarheid en hanteerbaarheid van de katheter. In vitro werd de beeldvorming van bismutdeeltjes beoordeeld met behulp van microCT en klinische dual-energy CT (DECT). Bij DECT wordt een aanvullende verzwakkingsmeting verkregen met een tweede röntgenspectrum en gedetecteerd met één detector met verschillende spectrale gevoeligheden of met twee detectoren, waardoor verschillende materialen kunnen worden onderscheiden op basis van de energieabsorptie van hun K-shell elektronen ( $k$-edge energie). Bovendien kan zo ook de massadichtheid van twee of drie materialen in een mengsel met bekende elementaire samenstelling worden gekwantificeerd [65] 
(figuur 6.1). Wij hebben vastgesteld dat onze nieuwe microdeeltjes op basis van bismut met behulp van DECT gemakkelijk te onderscheiden zijn van het vloeibare contrastmiddel in suspensie (jodium) bij blootstelling aan energiedoses van 80 en $150 \mathrm{kVp}$. Dit als gevolg van verschillen in k-edge energie tussen bismut en jodium, een kenmerk dat de follow-up tijdens en na TACE van HCC mogelijk maakt. Op grond van de chemische samenstelling van de bismutdeeltjes wordt verwacht dat de laad- en afgifteprofielen vergelijkbaar zijn met die van de voorganger, de jodiumhoudende deeltjes. Verder in vivo preklinisch onderzoek is nodig om de radiopaciteit van deze nieuwe deeltjes verder te onderzoeken en hun embolisatie, hanteerbaarheid, samendrukbaarheid, opschuifbaarheid en geneesmiddel elutieeigenschappen in doelweefsel en niet-doelweefsels te beoordelen. 



\section{Abbreviations}

DCC

DCM

$\mathrm{DiO}$

DIPEA

DPPC

DSPE-PEG 2000
$N, N^{\prime}$-Dicyclohexylcarbodiimide

Dichloromethane

3,3'-Dioctadecyloxacarbocyanine perchlorate $N, N$-Diisopropylethylamine 1,2-Dipalmitoyl-sn-glycero-3-phosphocholine 1,2-Distearoyl-sn-glycero-3-phosphoethanolamine-N[methoxy(polyethylene glycol)-2000] (ammonium salt)

DSPE-PEG ${ }_{2000}$ carboxylic acid 1,2-Distearoyl-sn-glycero-3-phosphoethanolamine-N[carboxy(Polyethylene Glycol)2000] (ammonium salt)

HATU $N, N, N^{\prime}, N^{\prime}$-Tetramethyl-O-(7-azabenzotriazol-1-yl) uronium hexafluorophosphate

$\mathrm{HOBt}$ 1-Hydroxybenzotriazole hydrate

cKNGRE

LTSL

TSL

MSPC

NMP

NGR

OG

TFA

PDGFR

VEGFR

Equiv

NMR

CT

DECT cyclic KNGRE

Lysolipid-containing temperature sensitive liposome Temperature sensitive liposome 1-Stearoyl-2-hydroxy-sn-glycero-3-phosphocholine $\mathrm{N}$-methyl-2-pyrrolidinone Asp-Gly-Arg

Oregon Green ${ }^{\circledR} 488$ carboxylic acid, succinimidyl ester Trifluoroacetic acid Platelet-derived growth factor receptor Vascular endothelial growth factor receptor Equivalent Nuclear Magnetic Resonance Computed Tomography Dual Energy Computed Tomography 



\section{List of publications}

1. Formulation and characterization of magnetic resonance image-able thermally sensitive liposomes for use with magnetic resonance-guided high intensity focused ultrasound International Journal of. Hyperthermia, 2011.

2. Synthesis and in vitro evaluation of novel cyclic NGR peptide targeted thermally sensitive liposome Journal of Controlled Release, 2010

3. Synthesis and characterization of image-able polyvinyl alcohol microspheres for image-guided chemoembolization Journal of Materials Science: Materials in Medicine, 2015

4. Synthesis, characterization, and imaging of radiopaque bismuth beads for image-guided transarterial embolization

Scientific Reports, 2021 



\section{Acknowledgements}

I have not come along this far all by myself. Several people have contributed to my success in life in general and in my thesis work in particular. Professors Storm Gert, Chrit Moonen and Dr. Bradford J Wood are among the many great people in the front line who have made this a reality. Because of you, opportunity has become possibility and then a reality, therefore, I kindly express my heartfelt gratitude to you all.

Brad, you said once, "I am supportive of Ayele's PhD...." The most powerful words that I concurred with. Even though, I do not know when but was sure I am getting my $\mathrm{PhD}$. Thank you Brad!

Getting done with my $\mathrm{PhD}$ degree was an inspiration installed by my late father, Hailu Negussie, when I was a child. He used to say "my son is going to be a doctor." This inspirational word has been revived and become a wish and later a possibility. I dared to express my wish to Dr. Matthew Dreher who listened to my desire and said "we will take you there". Today this desire has been fulfilled therefore, I am gladly express my sincere thankfulness to my former supervisor, Dr. Matthew Dreher, who has trusted me to reduce his ideas for some of the projects in the thesis into practices.

I am greatly indebted to Drs. Pavel Yarmolenko, Jenna L. Miller, Ashish Ranjan, Carmen Gacchina, Quirina De Ruiter, Ari Partanen, Karun Sharma, Victor Frenkel, Gouthan Reddy, Steven Drake, Andrew Lewis, Hugh Britton, Yiqing Tang and Mrs. Genevieve Jacobs and Mr. David Woods who were involved in 
various capacity throughout the projects and greatly shaped the outcome of my works.

Especial thanks to my dear friend Dr. Sander Langereis for reading, commenting my thesis and writing the thesis summary in Dutch.

There is a saying "Time and tide wait for none", but I would say there is a coincidence for especial moment (time) and especial people (tide) with a potential to shape the course of someone's destiny. In this regard, I would appreciate the moment I had with the then research fellow at NIH, Elma Hogeboom who rerouted my academic destiny. Thank you, Elma!

The path to my $\mathrm{PhD}$ was paved when I got the opportunity to work at NIH. The door to NIH was wide opened and has led me to meet great people like Drs. Wood, Dreher, Professors Storm, Moonen and others. This opportunity now turned into a successful possibility. I am indebted to Dr Narasimhan Danthi my former supervisor, who gave my first position to work as a chemist at Molecular and Imaging Laboratory (MIL), NIH.

Opportunity is like pop quiz that pops up but readiness to handle and utilize the obtained opportunity only makes a difference. I am indebted to my former chemistry teachers and research advisors from Addis Ababa University particularly to Dr. Gizachew Alemayhu and professor Berhanu Abegaz as well as former chemistry teachers from University of Oslo especially, to Professors Einar Uggerud and Karl Egil Malterud and former research advisor professor Frode Rise and Lise-Lotte Gundersen who made me fit to carry out this endeavor. Especial appreciation should go to Professor Uggerud for his support in a difficult time. 
No matter how well I am doing "NOW", it is difficult to be where I am unless dedication, support and love has not been offered to me from the mother of my beautiful kids, Mulu Birke. Mulyee!, you have given us (Ruhama, Aaron, Abbey, Isabella and myself) your entire life and love unreserved. Thank you!

My sisters Messi and Etaba, brothers Endu and Teddi thank you all for the great wishes you all have.

Last but not least, I am grateful to Fantish and Meaza who were with me in my dream and pursuit of higher learnings. I am successful because of you so I thank you and love you much! 



\section{Curriculum Vitae}

Ayele H Negussie was born on $28^{\text {th }}$ of January 1966 in Addis Ababa, Ethiopia. In 1989 and 1995, obtained his BSc. and MSc degrees in Chemistry from Faculty of Sciences, Department of Chemistry, Addis Ababa University, Addis Ababa, Ethiopia, respectively. He then joined the Faculty of Mathematics and Natural sciences, University of Oslo, Norway for post graduate program in Synthetic Organic Chemistry where he obtained Candidatus Scientiarum in 2004. In 2005, he joined Molecular Imaging Laboratory, Radiology \& Imaging Science Department, Clinical Center, National Institutes of Health (NIH), USA where the research interest was developing imaging probe to diagnose Alzheimer disease. In 2006, he has joined Wood Lab, Radiology \& Imaging Science Department, CC, NIH, USA as a chemist where he actively involved in the development of thermally sensitive targeted liposomes, image-able liposome and image-able beads for tumor targeted imaging and therapy. While working at Wood Lab, NIH, he joined the Department of Biomaterials Science and Technology, University of Twente, Enschede, The Netherlands to pursue for his $\mathrm{PhD}$ under the supervision of Professor Gert Storm and Professor Chrit Moonen (Center for Imaging Sciences, Imaging Division, University Medical Center Utrecht, Utrecht, The Netherlands). His major research interests are the design and synthesis of receptor targeted thermally sensitive liposomes, thermally sensitive image-able liposomes and image able beads for tumor targeted imaging and therapy. 
